Copyright (c) 2004 by Heather Kay Gerberich All rights reserved 


\title{
SEARCh For Excited or Exotic Electron Production Using the Dielectron + Photon Signature AT CDF IN Run II \\ by
}

\author{
Heather Kay Gerberich \\ Department of Physics \\ Duke University
}

Date:

Approved:

Prof. Ashutosh Kotwal, Supervisor

Prof. Alfred Goshaw

Prof. Calvin Howell

Prof. Seog Oh

Prof. Ronen Plesser

Dissertation submitted in partial fulfillment of the requirements for the degree of Doctor of Philosophy in the Department of Physics

in the Graduate School of

Duke University

2004 


\title{
$\underline{\text { ABSTRACT }}$ \\ (Physics) \\ SeARCh FOR Excited or Exotic Electron \\ Production Using the Dielectron + Photon \\ Signature at CDF in Run II
}

by

\author{
Heather Kay Gerberich \\ Department of Physics \\ Duke University
}

Date:

Approved:

Prof. Ashutosh Kotwal, Supervisor

Prof. Alfred Goshaw

Prof. Calvin Howell

Prof. Seog Oh

Prof. Ronen Plesser

An abstract of a dissertation submitted in partial fulfillment of the requirements for the degree of Doctor of Philosophy

in the Department of Physics

in the Graduate School of

Duke University

2004 


\section{Abstract}

We present a search for excited or exotic electrons decaying to an electron and a photon with high transverse momentum. An oppositely charged electron is produced in association with the excited electron, yielding a final state dielectron + photon signature. The discovery of excited electrons would be a first indication of lepton compositeness.

We use $\sim 202 \mathrm{pb}^{-1}$ of data collected in $p \bar{p}$ collisions at $\sqrt{s}=1.96 \mathrm{TeV}$ with the Collider Detector at Fermilab during March 2001 through September 2003. The data are consistent with standard model expectations. Upper limits are set on the experimental cross-section $\sigma\left(\bar{p} p \rightarrow e e^{*} \rightarrow e e \gamma\right)$ at the $95 \%$ confidence level in a contact-interaction model and a gauge-mediated interaction model. Limits are also presented as exclusion regions in the parameter space of the excited electron mass $\left(M_{e^{*}}\right)$ and the compositeness energy scale $(\Lambda)$. In the contact-interaction model, for which there are no previously published limits, we find $M_{e^{*}}<906 \mathrm{GeV}$ is excluded for $M_{e^{*}}=\Lambda$. In the gauge-mediated model, the exclusion region in the $M_{e^{*}}$ versus the phenomenological coupling $f / \Lambda$ parameter space is extended to $M_{e^{*}}<430 \mathrm{GeV}$ for $f / \Lambda \sim 10^{-2} \mathrm{GeV}^{-1}$. In comparison, other experiments have excluded $M_{e^{*}}<280$ $\mathrm{GeV}$ for $f / \Lambda \sim 10^{-2} \mathrm{GeV}^{-1}$. 


\section{Acknowledgements}

First, I thank my advisor, Ashutosh Kotwal, for his guidance, patience, understanding, sense of humor, kindness, and, among other things, not letting me quit and helping me understand and learn throughout this analysis. Without his encouragement and being the type of person he is, I may not have completed this project. Also I thank Al Lee, who insisted I spend one semester working for Ashutosh before making any decision about my future in physics at Duke. Were it not for that semester, I would not be where I am today. I thank the members of the Duke HEP group for providing a friendly and productive work environment.

The graduate students, post-docs, and professors at both Duke and CDF have been very helpful. Thanks to Mike for his comic abilities, the $Z+\gamma$ Monte Carlo, and helping me figure out things without making me feel stupid for asking. Thanks to Chris for his efforts to make me panic shortly before every presentation and also for his helpful comments. Also, the exotics, vegy, electron task force, and photon working group were very helpful in the completion of this analysis.

I credit my high school chemistry and physics teacher, Mr. Copenhaver, for stimulating my interest in science. I thank the physics professors at Clemson University, especially Prof. Daw, Prof. Gilreath, and Prof. Turner, who always supported and encouraged my physics endeavors during my college years.

I thank my close friends for their support, friendship, and efforts to keep me somewhat sane, including Brandon, Carol, Christian, Linda, Mike, Mike, Monty... 
Of course, I thank my four-legged furry friends who were a source of joy, support, and companionship throughout my graduate career.

I also thank my entire family for their love and support. In particular, my grandmother, Edythe Gould, to whom this thesis is dedicated. She supported me and my choices throughout my life until she passed my first year at Duke, and I am eternally grateful for her influences on me and my life.

Finally, I am grateful to all members of the CDF collaboration and Fermilab whose hard work and dedication make the experiment possible. 
In Memory of Frank and Edythe Gould 


\section{Contents}

Abstract $\quad$ iv

List of Figures $\quad$ xviii

List of Tables $\quad$ xxvii

1 Introduction 1

1.1 The Standard Model . . . . . . . . . . . . . . . . . . . . 1

1.1.1 Fundamental Constituents of Matter . . . . . . . . 2

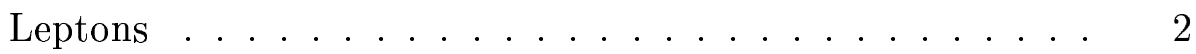

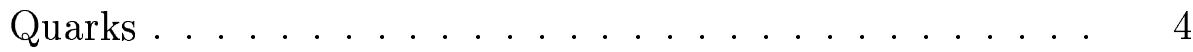

1.1.2 Gauge Bosons and Forces . . . . . . . . . . . 6

Quantum Chromodynamics ............. 7

Electroweak Model . . . . . . . . . . . . . 8

1.1.3 $Z+\gamma, Z+Z$, and $W+Z$ Production $\ldots \ldots \ldots \ldots$

1.1.4 Parton Distribution Functions . . . . . . . . . . . . 14

1.2 Natural Units . . . . . . . . . . . . . . . . . . 16

2 Excited/Exotic Electrons $\quad 17$

2.1 Excited Electron Models . . . . . . . . . . . . . . 17

2.1.1 Contact Interaction Model . . . . . . . . . . . . . 18

2.1.2 Gauge Mediated Model . . . . . . . . . . . . . . . 19 
$2.2 e^{*}$ Decay Modes . . . . . . . . . . . . . . 22

$2.2 .1 e^{*}$ Partial Widths . . . . . . . . . . . . 23

$2.2 .2 e^{*} \rightarrow e \gamma$ Decay Channel . . . . . . . . . . . . . 23

2.3 Monte Carlo Samples . . . . . . . . . . . . . . . . . 25

2.3 .1 Contact Interaction Model . . . . . . . . . . . . 26

2.3.2 Gauge Mediated Model . . . . . . . . . . . . . . 26

2.4 Excited Electron Properties . . . . . . . . . . . . . . 27

2.4 .1 Invariant Mass Properties $\ldots \ldots \ldots \ldots 27$

$2.4 .2 \quad$ Kinematic Properties . . . . . . . . . . . . . . . . 29

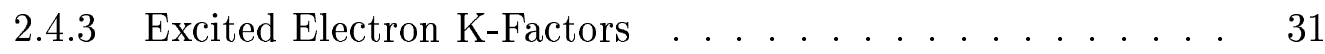

3 Fermilab Accelerator and CDF Detector 36

3.1 Accelerator . . . . . . . . . . . . . 36

3.2 Collider Detector at Fermilab . . . . . . . . . . . . . 38

3.2 .1 Geometrical Coordinate System . . . . . . . . . . . . 39

3.2 .2 Tracking System . . . . . . . . . . . . . 40

Silicon Tracking . . . . . . . . . . . . . . 4 41

Central Outer Tracker . . . . . . . . . . . . . 42

3.2.3 Calorimetry System . . . . . . . . . . . . . . . . 46

Central Calorimeter . . . . . . . . . . . . 47

Plug Electromagnetic and Hadronic Calorimeter . . . . . . 50

3.2.4 Central Electromagnetic Pre-Radiator Chambers _.... 50

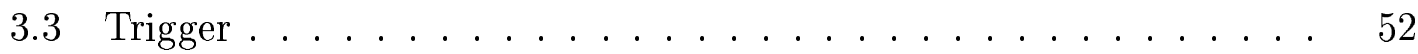

$3.3 .1 \quad$ Level $1 \ldots \ldots \ldots \ldots \ldots \ldots \ldots$

$3.3 .2 \quad$ Level $2 \ldots \ldots \ldots \ldots \ldots \ldots \ldots$ 
$3.3 .3 \quad$ Level $3 \ldots \ldots \ldots \ldots \ldots$. . . . . . . . . . . . 54

3.4 Detector Simulation . . . . . . . . . . . . . . 54

4 Dataset and Event Selection $\quad 57$

4.1 Triggers $\ldots \ldots \ldots \ldots \ldots \ldots \ldots \ldots \ldots \ldots \ldots$

4.1.1 ELECTRON_CENTRAL_18 Trigger Path . . . . . . . 58

4.1.2 ELECTRON70_L2_JET Trigger Path . . . . . . . . . . 59

4.1.3 JET_20, JET_50, JET_70, and JET_100 Trigger Paths . . . 60

4.2 Central and Plug Electromagnetic Clustering Algorithms . . . . . 61

4.3 CDF Electron and Photon Objects . . . . . . . . . . 62

4.4 Central Electron and Photon Identification . . . . . . . . . . 63

4.5 Central Electron Identification . . . . . . . . . . . . . 67

4.6 Central Photon Identification $\ldots \ldots \ldots \ldots$

4.7 Plug Electron and Photon Identification $\ldots \ldots \ldots \ldots . \ldots 71$

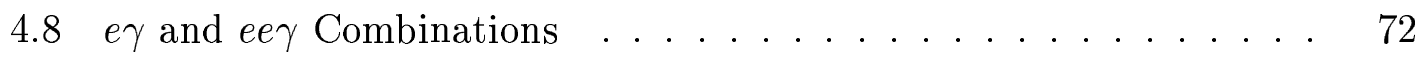

$5 \quad$ Energy Corrections $\quad 73$

5.1 Energy Scales . . . . . . . . . . . . . . . . 74

5.1.1 Central Electrons and Photons . . . . . . . . . 74

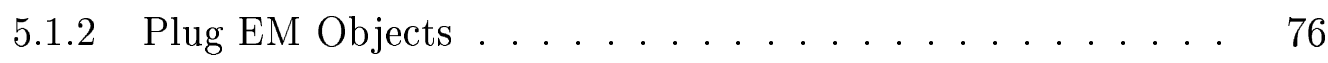

5.2 Energy Resolution $\ldots \ldots \ldots \ldots \ldots \ldots \ldots$

5.2 .1 Central Electrons and Photons $\ldots \ldots \ldots \ldots 78$

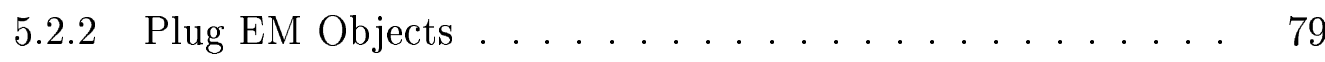

5.3 Systematic Uncertainty on Energy Corrections . . . . . . . . . . 81

5.3.1 Central Electron and Photon Energy Scale Systematic Uncer-

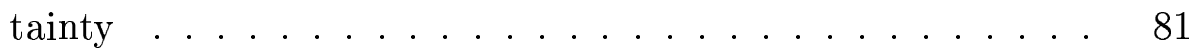


5.3.2 Central Electron and Photon Energy Resolution Systematic Uncertainty . . . . . . . . . . . . . . . . . . 82

5.3.3 Plug EM Object Energy Scale Systematic Uncertainty _. . 82

5.3.4 Plug EM Object Resolution Systematic Uncertainty . . . . . 83

5.4 Summary $\ldots \ldots \ldots \ldots \ldots \ldots \ldots \ldots$

6 Efficiency $\quad 85$

6.1 Identification Efficiencies $\ldots \ldots \ldots \ldots \ldots \ldots$

6.1.1 Central Electron Identification Efficiency . . . . . . . . 86

Measurement . . . . . . . . . . . . 86

$N-1$ Identification Efficiency $\ldots \ldots \ldots \ldots$

$N-1$ Efficiency Distributions . . . . . . . . . 88

Summary . . . . . . . . . . . . . . 89

6.1.2 Central Photon Identification Efficiency . . . . . . . . . . 89

Measurement . . . . . . . . . . . . . . . 89

Effect of Bremsstrahlung . . . . . . . . . . . . . 94 94

$N-1$ Identification Efficiency $\ldots \ldots \ldots \ldots . \ldots 95$

"Emulated" $N-1$ Identification Distributions . . . . . . 96

Summary . . . . . . . . . . . . . . . 99

6.1.3 Plug EM Object Identification Efficiency . . . . . . . . . 99

6.1 .4 Measurement . . . . . . . . . . . . . . . . 99

6.1 .5 Summary . . . . . . . . . . . . . . . . . 104

6.2 Trigger Efficiency $\ldots \ldots \ldots \ldots \ldots \ldots$

$6.3 \quad Z$ Vertex Efficiency . . . . . . . . . . . . . . . . . 108

6.4 Summary . . . . . . . . . . . . . . . . . . . 109 
$\begin{array}{lll}7 & \text { Signal Acceptance } & 110\end{array}$

7.1 Maximum Acceptance . . . . . . . . . . . . . . . . . 111

7.1.1 Contact Interaction $e^{*} \ldots \ldots \ldots \ldots 111$

$7.1 .2 \quad$ Gauge Mediated $e^{*} \ldots \ldots \ldots \ldots \ldots 11$

7.2 Total Signal Acceptance . . . . . . . . . . . . . . . . . . 112

7.2 .1 Contact Interaction $e^{*} \ldots \ldots \ldots \ldots$

7.2 .2 Gauge Mediated $e^{*} \ldots \ldots \ldots \ldots \ldots$

7.3 Contact Interaction Model Acceptance Uncertainties _. . . . . . 115

7.3.1 Statistical Error . . . . . . . . . . . . . . . . 115

7.3.2 Parton Distribution Functions . . . . . . . . . . . 117

7.3.3 Extra Minimum-Bias Events . . . . . . . . . . . . . 117

7.3.4 Identification Efficiency . . . . . . . . . . . . . . . . . 121

7.3 .5 Trigger Efficiency . . . . . . . . . . . . . . . . 121

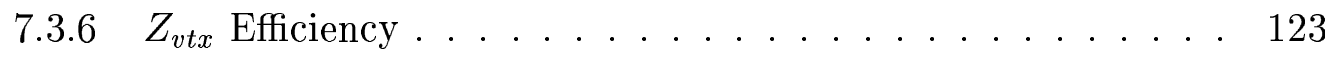

7.3.7 Extra Material in the Simulation . . . . . . . . . . 125

7.3 .8 Energy Scale . . . . . . . . . . . . . . 126

7.3.9 Energy Resolution . . . . . . . . . . . . . . . . 130

7.3 .10 Summary . . . . . . . . . . . . . . . . 132

7.4 Gauge Mediated Model Acceptance Uncertainties . . . . . . . . 133

7.4.1 Statistical Uncertainty . . . . . . . . . . . . . . 133

7.4 .2 Identification Efficiency . . . . . . . . . . . . . . 133

$7.4 .3 \quad$ Trigger Efficiency . . . . . . . . . . . . . . . . 133

7.4 .4 Energy Scale . . . . . . . . . . . . . . . . . 134

7.4.5 Energy Resolution . . . . . . . . . . . . . . . . 134 
$7.4 .6 \quad$ Summary . . . . . . . . . . . . . . . 137

7.5 Simulation of Electron + Photon Invariant Mass . . . . . . . . 140

8 Fake Rates 141

8.1 Unbiased Central Jet $\rightarrow$ Electron Fake Rate . . . . . . . . . 142

8.1.1 Data Samples . . . . . . . . . . . . . . . . . . 142

$8.1 .2 \quad$ Measurement . . . . . . . . . . . . . . . . . 143

$8.1 .3 \quad$ Systematics . . . . . . . . . . . . . . . 146

8.1.4 Jet $E_{T} \rightarrow$ Electron $E_{T}$ Fragmentation Function $\ldots \ldots . .147$

8.2 Trigger Electromagnetic Object $\rightarrow$ Electron Fake Rate . . . . . 148

8.2 .1 Data Samples . . . . . . . . . . . . . . . . . 148

8.2 .2 Measurement . . . . . . . . . . . . . . . 150

8.2 .3 Measurement Uncertainty . . . . . . . . . . . . . 152

8.3 Unbiased Central Jet $\rightarrow$ Photon Fake Rate . . . . . . . . . . 154

8.3.1 Raw Fake Rate . . . . . . . . . . . . . . 155

Data Samples . . . . . . . . . . . . . 155

Measurement . . . . . . . . . . . . . 155

8.3.2 Prompt Photon Subtraction . . . . . . . . . . . . . 157

Data Samples . . . . . . . . . . . . . 157

Prompt Photon Fraction Measurement Using the CPR . . 158

CPR Detection Efficiency . . . . . . . . . . . 160

Underlying Event Fluctuations in the CPR . . . . . . . 164

True Conversion Rate . . . . . . . . . . . . 166

Expected Conversion Probabilities . . . . . . . . . . . 168

Prompt Photon Fraction . . . . . . . . . . . . 170 
8.3.3 Corrected Jet $\rightarrow \gamma$ Fake Rate . . . . . . . . . 171

Comparisons of Results to an Alternate CES Method . . . . 172

8.3.4 Systematics . . . . . . . . . . . . . . . . 175

8.3.5 Jet $E_{T} \rightarrow \gamma E_{T}$ Fragmentation Function $\ldots \ldots \ldots \ldots$

8.4 Unbiased Plug Jet $\rightarrow$ Electromagnetic Object Fake Rate . . . . . 180

8.4 .1 Data Samples . . . . . . . . . . . . . . . . 180

8.4 .2 Measurement . . . . . . . . . . . . . . . . 182

$8.4 .3 \quad$ Systematics . . . . . . . . . . . . . . . . 183

8.4.4 Jet $E_{T} \rightarrow$ Plug EM Object $E_{T}$ Fragmentation Function . . . 184

9 Backgrounds 190

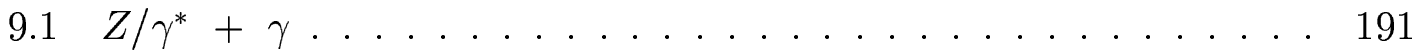

9.1.1 $Z_{\gamma}$ Background Prediction . . . . . . . . . . . . 192

$9.1 .2 \quad Z \gamma$ Background Systematics . . . . . . . . . . . . . 193

Parton Distribution Function Uncertainty on Acceptance . . 193

Parton Distribution Function Uncertainty on Cross-section . 193

Extra Material in the Simulation . . . . . . . . . . . . 194

Central Electron Identification Efficiency . . . . . . . . . . 194

Central Photon Identification Efficiency . . . . . . . . . . 194

Plug EM Object Identification Efficiency . . . . . . . . . 195

Trigger Efficiency . . . . . . . . . . . . . . . 195

$Z_{v t x}$ Efficiency . . . . . . . . . . . . . 195

Energy Scale . . . . . . . . . . . . . . . 196

Energy Resolution . . . . . . . . . . . . . . . 196

9.1 .3 Summary . . . . . . . . . . . . . . . . . . . 197 
$9.2 \quad Z(\rightarrow e e)+$ jet Background $\ldots \ldots \ldots \ldots \ldots \ldots$

9.2.1 Central Region Measurement _. . . . . . . . . . 198

9.2 .2 Plug Region Measurement . . . . . . . . . . . . . . . 199

9.2.3 Z $(\rightarrow e e)+$ jet Background Prediction . . . . . . . . . 199

9.2.4 Z $(\rightarrow e e)+$ jet Background Systematics _ . . . . . . . 199

$9.3 \quad W(\rightarrow e \nu) Z(\rightarrow e e)$ Background . . . . . . . . . . . . 200

9.3.1 $W(\rightarrow e \nu) Z(\rightarrow e e)$ Background Prediction . . . . . . 201

9.3.2 W( $\rightarrow e \nu) Z(\rightarrow e e)$ Background Systematics . . . . . . . 201

$9.4 \quad Z(\rightarrow e e) Z(\rightarrow e e)$ Background . . . . . . . . . . . 201

9.4.1 $Z(\rightarrow e e) Z(\rightarrow e e)$ Background Prediction . . . . . . . 201

9.4.2 Z $(\rightarrow e e) Z(\rightarrow e e)$ Background Systematics . . . . . . . 202

9.5 Multijet Background . . . . . . . . . . . . . . . . . 202

9.5.1 Central Region Measurement . . . . . . . . . . . 202

9.5.2 Plug Region Measurement . . . . . . . . . . . . . 203

9.5.3 Multijet Background Prediction . . . . . . . . . . . . 204

9.5.4 Multijet Background Prediction Systematics . . . . . . . 205

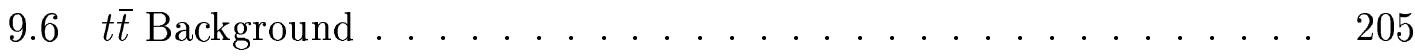

9.6.1 $t \bar{t}$ Background Prediction . . . . . . . . . . . 205

$9.6 .2 t \bar{t}$ Background Systematics . . . . . . . . . . . 206

9.7 Diphoton + jet Background $\ldots \ldots \ldots \ldots \ldots$

9.7 .1 Central Region . . . . . . . . . . . . 206

9.7 .2 Plug Region . . . . . . . . . . . . . . . 207

9.7.3 Diphoton + jet Background Prediction . . . . . . . . 207

9.7.4 Diphoton + jet Background Systematics . . . . . . . 208 
$9.8 W(\rightarrow e \nu)+$ jets Background $\ldots \ldots \ldots \ldots \ldots \ldots$

9.8.1 Central Region Measurement _. . . . . . . . . . 208

9.8.2 Plug Region Measurement . . . . . . . . . . . . . 209

9.8.3 $W(\rightarrow e \nu)+$ Jets Background Prediction . . . . . . . 210

9.8.4 W( $\rightarrow e \nu)+$ Jets Background Systematics . . . . . . 210

9.9 Background Prediction from All Sources . . . . . . . . . . . . 210

9.10 Effect of Dielectron Invariant Mass Cut . . . . . . . . . . . 212

10 Discussion of Candidate Events $\quad 216$

10.1 Introduction $\ldots \ldots \ldots \ldots \ldots \ldots \ldots \ldots \ldots \ldots$

10.2 Phoenix Tracking . . . . . . . . . . . . . . 216

10.3 Run 144674 , Event 4143240 . . . . . . . . . . . . . . . . 217

10.4 Run 147806 , Event 1167222 . . . . . . . . . . . . . . . . 221

10.5 Run 167866 , Event 443088 . . . . . . . . . . . . . . . . 223

10.6 Integrated Background Distributions Compared to the Data . . . 225

11 Extraction of Limits $\quad 228$

11.1 Introduction . . . . . . . . . . . . . . . . 228

11.2 Bayes Theorem . . . . . . . . . . . . . . . 228

11.2.1 Definition . . . . . . . . . . . . . . 229

11.2.2 Bayes Method Without Uncertainties . . . . . . . . . 230

Confidence Level . . . . . . . . . . . . . . . . 231

11.2.3 Bayesian Method Incorporating Uncertainties . . . . . . . 232

11.3 Background Prediction . . . . . . . . . . . . . . . 233

11.3.1 Search Region . . . . . . . . . . . . . . . . . 233 
11.3.2 Background Contribution . . . . . . . . . . . 234

11.4 Uncertainties . . . . . . . . . . . . . . . 235

11.4.1 Background Uncertainty . . . . . . . . . . . . . 235

11.4.2 Relative Error on Acceptance . . . . . . . . . . . . 236

11.4.3 Additional Systematics for Limit Calculations . . . . . . . 236

Luminosity . . . . . . . . . . . . . . 236

Uncertainty on Theoretical Cross-section Due to PDFs . . . 237

Uncertainty on Theoretical Cross-section due to K-factor . . 238

11.4.4 Total Uncertainty Due to Acceptance, Luminosity and Theoretical Sources . . . . . . . . . . . . . . 238

11.5 Theoretical Cross-section . . . . . . . . . . . . . . . 239

11.6 Limit Calculation . . . . . . . . . . . . . . . . . 240

11.7 Expected Mass Limits . . . . . . . . . . . . . . . . . . 240

11.8 Experimental Limits . . . . . . . . . . . . . . . . . . . . 241

11.8.1 Limit Results for $M_{e^{*}}=\Lambda \ldots \ldots \ldots \ldots \ldots$. . . . . 241

11.8.2 2-Dimensional Exclusion Regions . . . . . . . . . . 243

Contact Interaction Model . . . . . . . . . . . . . . 243

Gauge Mediated Model . . . . . . . . . . . . . . 245

$\begin{array}{ll}12 \text { Summary } & 248\end{array}$

$\begin{array}{ll}\text { Bibliography } & 251\end{array}$

$\begin{array}{ll}\text { Biography } & 254\end{array}$ 


\section{List of Figures}

1.1 Tree level Feynman diagrams for $Z+\gamma$ production. Figures (a) and (b) are production via initial state radiation in the t-channel and uchannels, respectively. Figure (c) is inner bremsstrahlung production (also called final state radiation). . . . . . . . . . . .

1.2 Tree level Feynman diagrams for $W+Z$ production. Figures (a) and (b) and (c) are t-channel, u-channel, and s-channel production, respectively. . . . . . . . . . . . . . .

1.3 Tree level Feynman diagrams for $Z+Z$ production. Figures (a) and (b) are t-channel and $\mathrm{u}$-channel production, respectively. . . . . .

2.1 Feynman diagram of contact interaction $e^{*}$ production. . . . . . .

2.2 Feynman diagram of gauge mediated $e^{*}$ production via $Z$ exchange.

$2.3 \quad$ Feynman diagram of $e^{*} \rightarrow e \gamma$ decay $\ldots \ldots \ldots \ldots$

2.4 Figure (a) shows the branching ratios for each $e^{*}$ decay channel as a function of $e^{*}$ mass. Figure (b) shows the the renormalized branching ratios for each $e^{*}$ decay channel after forcing the $W$ and $Z$ to decay leptonically to electrons or muons. . . . . . . . . . . . .

2.5 Parton level electron-photon invariant mass spectra for three choices of $M_{e^{*}}$ and $\Lambda \ldots \ldots \ldots \ldots \ldots \ldots \ldots$

2.6 Three body invariant mass distributions for three mass choices for the contact interaction model (a) and the gauge mediated model (b), normalized to unit area. . . . . . . . . . . . . . . . .

2.7 Parton level transverse energy distributions for the electron produced in association with the excited electron. . . . . . . . . . . .

2.8 Parton level transverse energy distributions for the electron from the excited electron decay. . . . . . . . . . . . . . 
2.9 Parton level transverse energy distributions for the photon from the excited electron decay. . . . . . . . . . . . . .

2.10 Parton level $\eta$ distributions for the electron produced in association with the excited electron. . . . . . . . . . . . . . .

2.11 Parton level $\eta$ distributions for the electron from the excited electron decay. . . . . . . . . . . . . . . . .

2.12 Parton level $\eta$ distributions for the photon from the excited electron

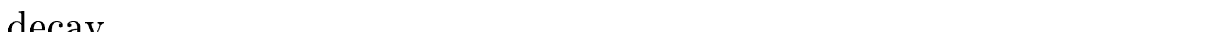

2.13 NNLO K-factors as a function of the system invariant mass. . . . .

2.14 NNLO K-factors as a function of $e^{*}$ mass for the gauge mediated and contact interaction excited electron models. . . . . . . . . . .

3.1 Schematic of accelerator components. . . . . . . . . . . . 37

3.2 Tevatron/CDF coordinate system . . . . . . . . . . . . 39

3.3 Longitudinal view of one quadrant of the CDF tracking system. . . 41

3.4 Schematic of SVX showing the 3 barrels placed end to end. . . . . 42

3.5 Figure (a) is an end view of silicon tracking system showing layer 00 + SVX + ISL making the $\phi$ segmentation apparent. The diameter of layer 00 is $2.2 \mathrm{~cm}$. Figure (b) shows an $r-Z$ view of the silicon detector layout. The ISL provides one additional silicon layer for matching SVX tracks to COT tracks and two layers for providing tracking coverage out to $\left|\eta_{d}\right|<2 \ldots \ldots \ldots \ldots \ldots$

3.6 East endplate schematic of the 4 axial and 4 stereo superlayers. The odd numbered layers are stereo and the even numbered layers are axial. Shown are the radii of the center of the superlayers in $\mathrm{cm}[\mathrm{in}]$.

3.7 Schematic of cell layout for superlayer 2 showing the wire layout for 3 of the cells and the $35^{\circ}$ tilt with respect to $\mathrm{R}$ to compensate for the Lorentz angle. . . . . . . . . . . . . . . . .

3.8 Schematic of one wedge of the central calorimeter. Each tower in the wedge is shown pointing back to the interaction point resulting in a projective geometry. . . . . . . . . . . . 
3.9 Schematic of one quadrant of plug detector system. . . . . . . . 51

3.10 Plug tower geometry. . . . . . . . . . . . . 52

3.11 Trigger system flow chart. . . . . . . . . . . 55

5.1 Figure (a) shows the dielectron mass distribution for $Z \rightarrow e e$ centralcentral candidate events in simulation and data before corrections. Figure (b) shows the average dielectron mass as a function of run number before corrections. . . . . . . . . . . . .

5.2 Figure (a) and (b) show the data-simulation comparisons for the $Z$ mass distribution for Central-Plug events before energy corrections, for the west and east barrels, respectively. Figure (c) and (d) show the average $Z$ mass peak as a function of run number, for the west and east barrels, respectively. . . . . . . . . . .

5.3 Figure (a) shows the dielectron mass distribution for $Z \rightarrow e e$ centralcentral candidate events in simulation and data after energy scale and smearing corrections. Figure (b) shows the average dielectron mass as a function of run number after energy scale corrections. . .

5.4 Figure (a) and (b) show the data-simulation comparisons for the $Z$ mass distribution for central-plug events after energy corrections, for the west and east barrels, respectively. Figure (c) and (d) show the average $Z$ mass peak as a function of run number, for the west and east barrels, respectively, after energy corrections. . . . . . .

$6.1 \quad N-1$ Electron identification distributions. . . . . . . . . . . 90

6.2 Electron identification efficiency as a function of $\eta_{d}, \phi, E_{T}$, and run

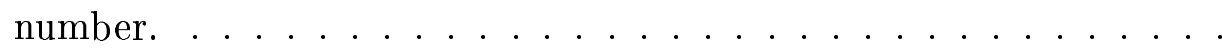

6.3 Electron identification efficiency as a function of $\eta_{d}, \phi$, and $E_{T}$ with no $L_{s h r}$ cuts. . . . . . . . . . . . . . . .

6.4 Emulated $N-1$ Photon Identification Distributions without $E / p$ cut. 97

6.5 Emulated $N-1$ Photon Identification Distributions with $E / p$ cut. . 98

6.6 Photon identification efficiency as a function of $\eta_{d}, \phi, E_{T}$, and run number with no $E / p$ cut. . . . . . . . . . . . 100 
6.7 $N-1$ Plug EM object identification distributions. Figures (a)(c) have no extra interactions. Figure (d) has a mean of 1 extra minimum-bias interaction per event. . . . . . . . . . 102

6.8 Plug EM object identification efficiency as a function of $\eta_{d}, \phi, E_{T}$, and run number. .................... 105

6.9 ELECTRON70_L2_JET trigger efficiency with respect to the ELECTRON_CENTRAL_18 trigger as a function of electron $E_{T} \ldots \ldots 7$

7.1 Maximum acceptance for contact interaction model. . . . . . . . 112

7.2 Figure (a) is the maximum acceptance for the gauge mediated $e^{*}$ production. Figure (b) shows the maximum acceptance for contact interaction $e^{*}$ production for $M_{e^{*}}$ up to $500 \mathrm{GeV}$ for comparison with the gauge mediated model. . . . . . . . . . . . .

7.3 Total signal acceptance for contact interaction model. . . . . . . . . 114

7.4 Figure (a) is the total signal acceptance for the gauge mediated $e^{*}$ production. Figure (b) shows the total signal acceptance for contact interaction $e^{*}$ production for $M_{e^{*}}$ up to $500 \mathrm{GeV}$ for comparison with the gauge mediated model. . . . . . . . . . . . . .

7.5 Plug EM object ID efficiency $\left(\epsilon_{p}\right)$ as a function of $E_{T}$. It is fit to $\epsilon=p_{0} \cdot\left(1-e^{p_{1} \cdot E_{T}}\right) \ldots \ldots \ldots \ldots$

7.6 Fully simulated electron-photon invariant mass spectra for three choices of $M_{e^{*}}$ and $\Lambda \ldots \ldots \ldots \ldots \ldots$. . . . . . . . . . . . . . . .

8.1 $\Delta R$ between jets and electron candidates. We use a cut of $\Delta R<0.15$ to match jets to electrons. . . . . . . . . . . . . . 144

8.2 Unbiased jet $\rightarrow$ electron fake rate as a function of $E_{T}$. The errors shown are statistical only. Shown are the quadratic fit results where $r_{e}=a+b \cdot E_{T}+c \cdot E_{T}^{2} \ldots \ldots \ldots \ldots$

8.3 Unbiased jet $\rightarrow$ electron fake rate as a function of detector $\eta$. The errors shown are statistical only. . . . . . . . . . . . 145 
8.4 Figure (a) shows the fake rate for Jet2 and Jet345 jets and the combined fake rate. Figure (b) shows the fit for the fake rate of Jet345 jets. The fit function is $r_{e}=a+b \cdot E_{T}+c \cdot E_{T}^{2}$. . . . . . 146

8.5 Figure (a) is the $E_{T}^{e} / E_{T}^{j}$ fragmentation function versus jet $E_{T}$. Figure (b) is the $E^{e} / E^{j}$ as a function of jet $\mathrm{E}$. The distributions are fit to $e^{p 0+p 1 \cdot E}+p 2 . \ldots \ldots \ldots \ldots \ldots \ldots$

8.6 Distributions of $E_{T}^{e} / E_{T}^{j}$ for different ranges of $E_{T}^{j}$. Figure (a) is for all $E_{T}^{j}$. Each is fit to a Gaussian. . . . . . . . . . . 149

8.7 Trigger EM object $\rightarrow$ electron fake rate as a function of $E_{T}$. The errors shown are statistical. The distribution is fit to a Gaussian plus a constant. The fit parameters are 0.52 for Gaussian norm, 25 $\mathrm{GeV}$ for Gaussian mean, 25.5 GeV for Gaussian sigma, and 0.45 for the constant. . . . . . . . . . . . . 153

8.8 Trigger EM object $\rightarrow$ electron fake rate as a function of detector $\eta$. The errors shown are statistical. . . . . . . . . . . . 153

8.9 Trigger EM object $\rightarrow$ electron fake rate as a function of run number. The errors shown are statistical. . . . . . . . . . . . . . 154

8.10 $\Delta R$ between jets and photon candidates. We use a cut of $\Delta R<0.15$ to match jets to photons. . . . . . . . . . . 156

8.11 Raw $j \rightarrow \gamma$ fake rate. . . . . . . . . . . 156

8.12 CPR $Q$ distributions for cosmic ray and $W \rightarrow \mu \nu$ muons and the photon candidates. The units of $\mathrm{Q}$ are fC. . . . . . . . . . 159

8.13 Predicted distribution of CPR $Q_{T}=Q \sin \theta$ for two collinear minimum-

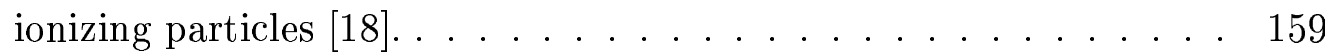

8.14 CPR $Q$ distribution for electrons from $W \rightarrow e \nu$ candidates. Note the difference in the $Q$ scale between electrons and the photon candidates (Figure 8.12) . . . . . . . . . . . . . . . 161

8.15 Dependence of CPR detector efficiency on barrel and module. . . . 162

8.16 Dependence of CPR detector efficiency on local $X$ (a) and local $Z$ (b).163

8.17 Distribution of $\Delta X$ between the local $X$ coordinate of the photon candidate's CES cluster and all CPR clusters in the same half-module.165 
8.18 Distribution of $\Delta X$ between the local $X$ coordinate of the muon track from $W \rightarrow \mu \nu$ sample and all CPR clusters in the same module. . . 166

8.19 Fraction of fakes in the jet $\rightarrow$ photon fake rate sample, $f_{\pi} \ldots \ldots 172$

8.20 Corrected $j \rightarrow \gamma$ fake rate. . . . . . . . . . . . . 173

8.21 Comparison between the raw $j \rightarrow \gamma$ fake rate measured in this CPRbased analysis and the CES-based analysis [25]. . . . . . . . 174

8.22 Comparison between the QCD fraction $f_{\pi}$ measured in this CPR based analysis and the CES based analysis [25]. . . . . . . . 174

8.23 Comparison between the corrected $j \rightarrow \gamma$ fake rate measured in this CPR based analysis and the CES based analysis [25]. . . . . . . 175

8.24 Raw $j \rightarrow \gamma$ fake rate measured as a function of detector $\eta$. . . 176

8.25 Raw $j \rightarrow \gamma$ fake rate measured in this CPR based analysis for jet 2, jets 345 and the combined fake rate. . . . . . . . . 177

8.26 Figure (a) is a profile of the $E_{T}^{\gamma} / E_{T}^{j}$ as a function of jet $E_{T}$. Figure (b) is a profile of $E^{\gamma} / E^{j}$ as a function of jet energy. . . . . . . .

8.27 Distributions of $E_{T}^{\gamma} / E_{T}^{j}$ for different ranges of $E_{T}^{j}$. Figure (a) is for all $E_{T}^{j}$. Each is fit to a Gaussian. . . . . . . . . . .

8.28 $\Delta R$ between jets and and plug EM object candidates. We use a cut of $\Delta R<0.2$ to match jets to plug EM objects. . . . . . . . 182

8.29 Plug jet $\rightarrow$ EM object fake rate as a function of detector $\eta \ldots \ldots$.

8.30 Plug jet $\rightarrow$ EM object fake rate as a function of $E_{T}$ for all $\eta$ regions as well as for the $1.2<\left|\eta_{d}\right|<1.7,1.7<\left|\eta_{d}\right|<2.3$, and $2.3<\left|\eta_{d}\right|<2.8$ regions separately. . . . . . . . . . . .

8.31 Fake rate distributions for $1.2<\left|\eta_{d}\right|<1.7$. Figure (a) shows the distribution for all jets and for Jet2 and Jet345. Figure (b) shows the fit for all jets, Figure (c) shows the fit for Jet345 jets. . . . . . .

8.32 Fake rate distributions for $1.7<\left|\eta_{d}\right|<2.3$. Figure (a) shows the distribution for all jets and for Jet2 and Jet345. Figure (b) shows the fit for all jets, Figure (c) shows the fit for Jet345 jets. . . . . . . 
8.33 Fake rate distributions for $2.3<\left|\eta_{d}\right|<2.8$. Figure (a) shows the distribution for all jets and for Jet2 and Jet345. Figure (b) shows the fit for all jets, Figure (c) shows the fit for Jet345 jets. . . . . . .

8.34 Figure (a) is a profile of the $E_{T}^{p e m} / E_{T}^{j}$ as a function of jet $E_{T}$. Figure (b) is a profile of $E^{p e m} / E^{j}$ as a function of jet energy. . . . . . .

8.35 Distributions of $E_{T}^{p e m} / E_{T}^{j}$ for different ranges of $E_{T}^{j}$. Figure (a) is for all $E_{T}^{j}$. Each is fit to a Gaussian. . . . . . . . . .

9.1 Figure (a) shows the individual background contributions from each source for the $M_{e \gamma}$ distribution. Figure (b) shows the summed background contributions for the $M_{e \gamma}$ distribution. . . . . . . . . 213

9.2 Figure (a) shows the individual contribution from each source of background for the $M_{e e \gamma}$ distribution. Figure (b) shows the summed background contributions for the $M_{e e \gamma}$ distribution. . . . . . .

9.3 Total background contribution from all sources. The red curve is the central value and the blue band is the error band. . . . . . . . . . 214

9.4 Dielectron invariant mass spectra for the two leading backgrounds, $Z+\gamma$ and $Z+$ jet. Events with $81<M_{e e}<101 \mathrm{GeV}$ are rejected. .

10.1 Event displays for Run=144674, Event=4143240. Figure (a) shows the COT event display with track $p_{T}>0.5 \mathrm{GeV}$. Figure (b) shows the calorimeter physics towers event display with tower $E_{T}>0.5 \mathrm{GeV} .219$

10.2 Phoenix track displays for run $=144674$, event $=4143240$. Figure (a) shows both $X$ vs $Z$ hypotheses (the red curves) and the best fit (negative charge, blue curve) for $p_{2}$. Figure (b) $x-z$ display shows the projected trajectories of the objects. . . . . . . . . . 220

10.3 Event displays for run=147806, event=1167222. Figure (a) shows the COT event display with track $p_{T}>0.5 \mathrm{GeV}$. Figure (b) shows the calorimeter physics towers event display with tower $E_{T}>0.5 \mathrm{GeV} .222$

10.4 Event displays for Run=167866 Event=443088. Figure (a) shows the COT event display with track $p_{T}>0.5 \mathrm{GeV}$. Figure (b) shows the calorimeter physics towers event display with tower $E_{T}>0.5 \mathrm{GeV} . .224$ 
10.5 Figure (a) shows the integrated background prediction from high mass to low mass for the $M_{e *}$ distribution. Figure (b) shows the integrated background prediction from high mass to low mass for the $M_{e e \gamma}$ distribution. The observed events are overlaid in black for run $=147806$, event $=1167222$, blue for run $=144674$, event $=4143240$, and magenta for $r u n=167866$, event $=443088 \ldots \ldots \ldots \ldots$

10.6 Figure (a) shows the integrated background prediction from high mass to low mass for the $M_{e e}$ distribution. Figure (b) shows the integrated background prediction from high energy to low energy for the $E_{T}^{\gamma}$ distribution. The observed events are overlaid in black for run $=147806$, event $=1167222$, blue for run $=144674$, event $=4143240$, and magenta for $r u n=167866$, event $=443088 \ldots \ldots \ldots \ldots$

11.1 This figure is an example of the confidence level. The region $N>N_{95}$ is excluded, where $N$ is the number of expected signal events. . . . 232

11.2 Uncertainty on $e^{*}$ cross-section due to PDFs as a function of $e^{*}$ mass. 237

11.3 Systematic uncertainty due to K-factor as a function of $e^{*}$ mass. Figure (a) is for the contact interaction model and Figure (b) is for the gauge mediated model. . . . . . . . . . . . . . 238

11.4 Total systematic uncertainty as a function of $e^{*}$ mass. Figure (a) is for the contact interaction model and Figure (b) is for the gauge mediated model. . . . . . . . . . . . . . . . . . 239

11.5 Figure (a) shows the expected limit for the gauge mediated model. Figure (b) shows the expected limit for the contact interaction model. 241

11.6 Figure (a) is the $M_{e^{*}}=\Lambda$ mass limit for the gauge mediated model. Figure (b) is the $M_{e^{*}}=\Lambda$ mass limit for the contact interaction model.

11.7 Experimental cross-section limits and theoretical cross-sections for the contact interaction and gauge mediated models, and the corresponding mass limits. . . . . . . . . . . . . .

11.8 The contact interaction model theoretical cross-sections for various values of $\Lambda$ and $M_{e^{*}}$ intersecting with the $95 \%$ confidence level upper limit on the experimental cross-section. . . . . . . . . . . . . 245 
11.9 Contact interaction model exclusion regions. Figure (a) is the $M_{e^{*}} / \Lambda-$ $M_{e^{*}}$ exclusion region. Figure (b) is the $1 / \Lambda-M_{e^{*}}$ exclusion region. 246

11.10Gauge mediated model exclusion region in the $f / \Lambda-M_{e^{*}}$ plane. Shown are the exclusion regions for CDF, L3, H1, and ZEUS. The black curve is the $\Gamma_{e^{*}}=2 \cdot M_{e^{*}}$ region above which the theory is excluded. $\Gamma_{e^{*}}$ is the full theoretical width. . . . . . . . . . 247

12.1 Expected limit for the gauge-mediated model for $M_{e^{*}}=\Lambda$ using $\sim 1 \mathrm{fb}^{-1}$ of data collected at CDF. . . . . . . . . 250 


\section{List of Tables}

1.1 Summary of lepton properties and quantum numbers: mass, spin, electric charge, electron lepton number $\left(L_{e}\right)$, muon lepton number $\left(L_{\mu}\right)$, tau lepton number $\left(L_{\tau}\right)$, lifetimes, primary decay channels, associated antiparticle, and the Standard Model forces each particle interacts with. The masses and lifetimes have been rounded. . . . . 3

1.2 Summary of quark properties: bare mass (Mass), electric charge, spin, and its antiparticle. . . . . . . . . . . . 4

1.3 Summary of weak isospin, electric charge, and weak hypercharge for leptons and quarks. . . . . . . . . . . . . .

1.4 Summary of gauge boson properties. The mass of the photon is given in $e V$, while the masses of the $W^{ \pm}$and $Z$ bosons are given in $G e V$.

1.5 Neutral vector and axial couplings to Standard Model fermions (quarks and leptons). . . . . . . . . . . . . . .

2.1 Neutral vector and axial couplings to Standard Model quarks. . . .

2.2 NLO and NNLO K-Factors for 10 mass choices. The NNLO uncertainty is computed from the fractional difference between the NLO

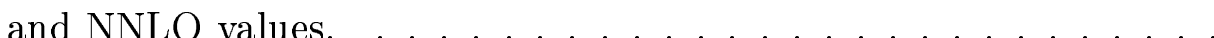

4.1 Names of triggers for each level of the JET_20, JET_50, JET_70, and JET_100 trigger paths. . . . . . . . . . . . 60

4.2 Central electron selection criteria. . . . . . . . . . . . 69

4.3 Central photon selection criteria. . . . . . . . . . . 71

4.4 Plug EM object selection criteria. . . . . . . . . . . 72

5.1 Energy scale factors applied to central EM objects . . . . . . . 75

xxvii 
5.2 Energy scale factors applied to west plug EM objects $\ldots \ldots \ldots 76$

5.3 Energy scale factors applied to east plug EM objects . . . . . . 78

5.4 Energy smearing applied to simulated plug EM objects . . . . . . 81

5.5 Summary of energy smearing applied to central electrons and photons. The energy smearing correction is $\left(3.17_{-0.22}^{+0.24}\right) \% \ldots \ldots \ldots$.

5.6 Summary of energy smearing applied to west plug EM objects. The energy smearing correction is $\left(1.9_{-0.7}^{+0.6}\right) \% \ldots \ldots \ldots \ldots$

5.7 Summary of energy smearing applied to east plug EM objects. The energy smearing correction is $\left(1.9_{-0.9}^{+0.7}\right) \% \ldots \ldots \ldots \ldots$

6.1 $N-1$ central electron identification efficiency. The errors shown are statistical. . . . . . . . . . . . . . .

6.2 Extra tight electron selection criteria used in the central photon and plug EM object identification studies. . . . . . . . . . .

6.3 Sequential efficiencies of photon cuts. Each cut is applied in addition to the previous cuts. The last line has the cumulative efficiency for electrons that behave most like photons. The errors shown are statistical. . . . . . . . . . . . . . . .

6.4 $N-1$ photon ID efficiency without the tight $E / p$ cut. The errors shown are statistical. . . . . . . . . . . . . 96

6.5 $N-1$ photon ID efficiency with the tight $E / p$ cut. The errors shown

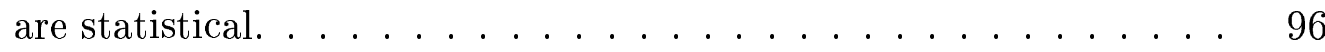

6.6 $N-1$ Plug EM Object Identification Efficiency using $Z \rightarrow e e$ centralplug events. . . . . . . . . . . . . . . . . . 101

6.7 $N-1$ Plug EM Object Identification Efficiency with and without extra minimum-bias interactions using $Z \rightarrow e e$ events. . . . . . 103

7.1 Relative statistical error on total signal acceptance for the contact interaction model. . . . . . . . . . . . . . 116

7.2 Relative difference between various PDF acceptances and the CTEQ5L acceptance. . . . . . . . . . . . . . . . . 118 
7.3 Plug EM object identification efficiency in simulated $e^{*}$ Monte Carlo with and without extra interactions. Also shown is the scale factor:

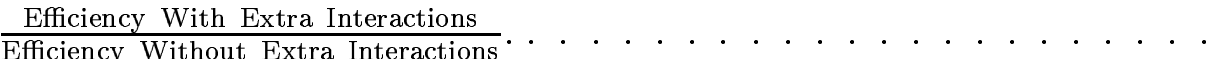

7.4 Relative systematic errors due to identification efficiencies on the contact interaction model total signal acceptance. . . . . . . . . .

7.5 Systematics on the contact interaction model total signal acceptance due to trigger efficiency uncertainty. Errors are relative. . . . . . . .

7.6 Systematics on the contact interaction model central acceptance due to extra material. . . . . . . . . . . . . . . . . . .

7.7 Systematics on the contact interaction model plug acceptance due to extra material. . . . . . . . . . . . . . . . 128

7.8 Systematics on the contact interaction model total (central+plug) acceptance due to extra material. . . . . . . . . . . . . . 129

7.9 Systematics on the contact interaction model total acceptance due to energy scaling. . . . . . . . . . . . . . . . .

7.10 Systematics on the contact interaction model total signal acceptance due to energy smearing corrections. . . . . . . . . . . . . .

7.11 Total signal acceptance and a summary of the uncertainties for the contact interaction model. All errors are $\triangle A / A$ in percentage. The bottom row shows the value used for $M_{e^{*}}>=125 \mathrm{GeV}$. For $M_{e^{*}}=$ $100 \mathrm{GeV}$, we use the value shown in the top row.

7.12 Relative statistical error on total signal acceptance for the gauge mediated model. . . . . . . . . . . . . . . . .

7.13 Relative systematic errors due to identification efficiencies for the gauge mediated model total signal acceptance. . . . . . . . . . .

7.14 Systematic uncertainties on the total signal acceptance due to trigger efficiency uncertainties for the Gauge Mediated model. . . . . . . . 136

7.15 Systematics on gauge mediated model total acceptance due to energy scaling. . . . . . . . . . . . . . . . .

7.16 Systematics on gauge mediated total signal acceptance due to energy smearing corrections. . . . . . . . . . . . . . 
7.17 Total signal acceptance and uncertainties for the gauge mediated model. All errors are $\Delta A / A$ in percentage. The bottom row shows the value used for $M_{e^{*}}>=110 \mathrm{GeV}$. For $M_{e^{*}}=100$ and $110 \mathrm{GeV}$, the value shown in the top row is used. . . . . . . . . . . . 139

8.1 Unbiased jet $\rightarrow$ electron fake rate in $E_{T}$ bins. . . . . . . . 147

8.2 Loose electron cuts for the $Z \rightarrow$ eerejection requirement in the trigger EM object $\rightarrow$ electron fake rate measurement. Events that have more than one EM cluster passing these cuts are rejected. . . . . . . 150

8.3 Trigger EM object $\rightarrow$ electron fake rate in $E_{T}$ bins. . . . . . 152

8.4 Raw $j \rightarrow \gamma$ fake rate. . . . . . . . . . . . . 156

8.5 Observed and true conversion rates for photon candidates. . . . . 168

8.6 Prompt photon fraction of the photon candidates. . . . . . . . 171

8.7 Uncertainty contributions on the QCD fraction $\left(f_{\pi}\right) . \delta f_{\pi} / f_{\pi}$ is given

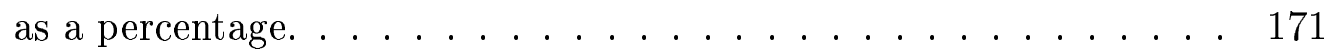

8.8 Corrected $j \rightarrow \gamma$ fake rate $\left(r_{\gamma}\right) \ldots \ldots \ldots \ldots \ldots \ldots \ldots$

8.9 Corrected $j \rightarrow \gamma$ fake rate $\left(r_{\gamma}\right) \ldots \ldots \ldots \ldots \ldots \ldots$

8.10 Raw $j \rightarrow \gamma$ fake rate, $f_{\pi}$, and corrected fake rate for Jet345 in the event. . . . . . . . . . . . . . . 178

8.11 Corrected $j \rightarrow \gamma$ fake rate $(r)$. We apply $f_{\pi}$ from the all jets measurement (jets $2,3,4,5)$ to the raw fake rates for Jet2 and Jet345 to obtain corrected fake rates. . . . . . . . . . . . 178

8.12 Corrected fake rate with asymmetric errors to account for the difference between the fake rate of Jet2 and Jet345 and the $\eta_{d}$ dependence, whichever is greater. . . . . . . . . . . . . .

9.1 Summary of systematic error contributions for the $Z \gamma$ background prediction. . . . . . . . . . . . . . . . . 198

9.2 Summary of the background prediction for the integrated number of events and associated errors. . . . . . . . . . . . 211 
9.3 Summary of integrated number of $e \gamma$ entries background prediction and associated errors. . . . . . . . . . . . . 212

10.1 Detailed information for run 144674 , event $4143240 \ldots \ldots$. . . . . 218

10.2 Event information for run=147806, event=1167222. $p_{2}$ is the fourth EM object in the event. . . . . . . . . . . . 221

10.3 Event information for $r u n=167866$, event $=443088 \ldots \ldots$. . . . .

11.1 Background prediction used in search regions for $M_{e^{*}}=\Lambda$. Units are

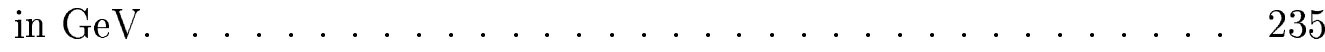

11.2 Number of observed events, relative uncertainty on acceptance and luminosity $(\triangle A / A)$, upper limit on the number of expected events using $\Delta A / A$, the upper limit on $\sigma$ at the $95 \%$ confidence level using $\Delta A / A$, total relative uncertainty $(\Delta L / L)$, upper limit on the number of expected events using $\Delta L / L$, and the upper limit on $\sigma$ at the $95 \%$ confidence level using $\Delta L / L$ for $M_{e^{*}}=\Lambda$ values between $100 \mathrm{GeV}$ and $1000 \mathrm{GeV}$ in the contact interaction model. . . . . . . . . . 242

11.3 Number of observed events, relative uncertainty on acceptance and luminosity $(\Delta A / A)$, upper limit on the number of expected events using $\triangle A / A$, the upper limit on $\sigma$ at the $95 \%$ confidence level using $\Delta A / A$, total relative uncertainty $(\Delta L / L)$, upper limit on the number of expected events using $\Delta L / L$, and the upper limit on $\sigma$ at the $95 \%$ confidence level using $\Delta L / L$ for $M_{e^{*}}=\Lambda$ values between $100 \mathrm{GeV}$ and $300 \mathrm{GeV}$ in the gauge mediated model. . . . . . . . . . 243 


\section{Chapter 1}

\section{Introduction}

The Standard Model of particle physics provides a description of our current understanding of the elementary constituents of matter and the forces which govern their behavior. In this analysis we search for events which could indicate a deviation from the Standard Model. Specifically, we search for excited or exotic electrons $\left(e^{*}\right)$ in the reaction:

$$
p+\bar{p} \rightarrow e^{*}+e \rightarrow e+\gamma+e
$$

This is a signature-based search for an excess of high transverse energy dielectron plus photon production at the Tevatron with a resonance in the electron-photon $(e \gamma)$ channel. We study the invariant mass of the $e \gamma$ combinations and use the search results to set limits on the production of excited or exotic electrons. Phenomenology of excited/exotic electrons is discussed further in Chapter 2.

\subsection{The Standard Model}

A natural starting point is a brief discussion of various components of the current Standard Model. In this section, we provide an overview of the fundamental constituents of matter and forces. 


\subsubsection{Fundamental Constituents of Matter}

The Standard Model categorizes the fundamental particles of matter in two categories: leptons and quarks.

\section{Leptons}

Leptons are spin 1/2 particles. There are six types of leptons organized into 3 generations (or families). The first generation consists of an electron $(e)$ and electronneutrino $\left(\nu_{e}\right)$, the second is a muon $(\mu)$ and mu-neutrino $\left(\nu_{\mu}\right)$, and the third generation is a $\operatorname{tau}(\tau)$ and tau-neutrino $\left(\nu_{\tau}\right)$. Each family has a lepton quantum number associated with it: $L_{e}, L_{\mu}$, and $L_{\tau}$, respectively. Table 1.1 shows a summary of the properties of leptons including their mass, spin, charge, lepton quantum numbers, lifetimes, primary decay channels (if any), corresponding antiparticle, and the forces with which they interact. The antiparticle has the same mass and spin as the associated particle but opposite sign of all the internal quantum numbers.

Parity describes the behavior of a system when it undergoes inversion, ie $\vec{r} \rightarrow$ $\overrightarrow{-r}, \vec{t} \rightarrow \vec{t}$. We introduce here the idea of "helicity" for spin $1 / 2$ particles. Helicity is defined as + if the particle spin is aligned with the direction of motion and - if the particle spin is aligned opposite the direction of motion. Particles with + helicity are "right" handed and particles with - helicity are "left" handed. Experiments have shown that the weak interaction violates conservation of parity and that all neutrinos (antineutrinos) are left (right) handed.

Associated with leptons are two additional quantum numbers: weak isospin $(T)$, whose third component is denoted as $T^{3}$ and weak hypercharge $(Y) . T^{3}$ and $Y$ are related to the electric charge $Q$ by $Q=T^{3}+\frac{Y}{2}$. Left handed leptons, including all neutrinos, have $T=1 / 2$ and $Y=-1$. Right handed leptons have $T=0$ and 
$Y=-2$. The electric charge, weak isospin and weak hypercharge for leptons are summarized in Table 1.3.

\begin{tabular}{|l|c|c|c|c|c|c|}
\hline & \multicolumn{2}{|c|}{ first generation } & \multicolumn{2}{c|}{ second generation } & \multicolumn{2}{c|}{ third generation } \\
\hline & $e^{-}$ & $\nu_{e}$ & $\mu^{-}$ & $\nu_{\mu}$ & $\tau^{-}$ & $\nu_{\tau}$ \\
\hline \hline Mass (MeV) $[2]$ & 0.5110 & $<0.0003$ & 105.7 & $<0.19$ & 1777 & $<18.2$ \\
\hline Spin & $1 / 2$ & $1 / 2$ & $1 / 2$ & $1 / 2$ & $1 / 2$ & $1 / 2$ \\
\hline Charge (e) & -1 & 0 & -1 & 0 & -1 & 0 \\
\hline$L_{e}$ & 1 & 1 & 0 & 0 & 0 & 0 \\
\hline$L_{\mu}$ & 0 & 0 & 1 & 1 & 0 & 0 \\
\hline$L_{\tau}$ & 0 & 0 & 0 & 0 & 1 & 1 \\
\hline Lifetime $[2]$ & $\infty$ & $\infty$ & $2.197 \mu s$ & $\infty$ & $291 f s$ & $\infty$ \\
\hline $\begin{array}{l}\text { Primary } \\
\text { Decay } \\
\text { Channels }\end{array}$ & -- & -- & $e^{-} \overline{\nu_{e}} \nu_{\mu}$ & -- & $\begin{array}{c}\mu^{-} \overline{\nu_{\mu}} \nu_{\tau}, \\
e^{-} \overline{\nu_{e}} \nu_{\tau},\end{array}$ & -- \\
\hline Antiparticle & $e^{+}$ & $\overline{\nu_{e}}$ & $\mu^{+}$ & $\overline{\nu_{\mu}}$ & $\rho^{+}$ & -- \\
\hline Forces & EM & Weak & EM & Weak & EM & Weak \\
& Weak & & Weak & & Weak & \\
\hline
\end{tabular}

Table 1.1: Summary of lepton properties and quantum numbers: mass, spin, electric charge, electron lepton number $\left(L_{e}\right)$, muon lepton number $\left(L_{\mu}\right)$, tau lepton number $\left(L_{\tau}\right)$, lifetimes, primary decay channels, associated antiparticle, and the Standard Model forces each particle interacts with. The masses and lifetimes have been rounded.

If neutrinos are massless particles, the lepton quantum numbers $\left(L_{e}, L_{\mu}\right.$, and $L_{\tau}$ ) are conserved in all interactions. Neutrinos oscillations such as $\nu_{e} \rightarrow \nu_{\mu}$ provide a means for measuring the mass difference between the three types of neutrinos and are also a clear violation of lepton number. Recent experiments at SNO (solar neutrino oscillations) and SuperKamiokande (atmospheric neutrino oscillations) successfully observed neutrino oscillations. These experiments indicate that neutrinos are massive and that the lepton quantum numbers are not conserved in weak interactions. 


\section{Quarks}

Like leptons, there are six flavors of quarks, split into three generations (or families). The first generation are the up $(u)$ and down $(d)$ quark. The charm $(c)$ and strange $(s)$ quarks comprise the second generation. The third family consists of the top $(t)$ and bottom $(b)$ quark. Quarks are spin 1/2 particles with electric charge $n / 3$, where $n$ is an integral depending on the type of quark. Table 1.2 summarizes the properties of the six types of quarks by their bare mass, spin, charge and associated antiparticle. Quarks interact via electromagnetic, weak, and strong interactions. Electromagnetic and strong interactions conserve quark flavor while quark flavor is not conserved in weak interactions.

\begin{tabular}{|l|c|c|c|c|}
\hline Quark & Mass (MeV) [2] & Spin & Charge (e) & Antiquark \\
\hline \hline$d$ & $2.5-5.5$ & $1 / 2$ & $-1 / 3$ & $\bar{d}$ \\
$u$ & $1.5-4.5$ & $1 / 2$ & $+2 / 3$ & $\bar{u}$ \\
\hline$s$ & $80-155$ & $1 / 2$ & $-1 / 3$ & $\bar{s}$ \\
$c$ & $1000-1400$ & $1 / 2$ & $+2 / 3$ & $\bar{c}$ \\
\hline$b$ & $4000-4500$ & $1 / 2$ & $-1 / 3$ & $b$ \\
$t$ & $174300 \pm 5100$ & $1 / 2$ & $+2 / 3$ & $\bar{t}$ \\
\hline
\end{tabular}

Table 1.2: Summary of quark properties: bare mass (Mass), electric charge, spin, and its antiparticle.

Like leptons, quarks have weak hypercharge $(Y)$ quantum number and weak isospin $(T)$. Again, the third component of isospin is related to the weak hypercharge and electric charge by $T^{3}=Q-\frac{Y}{2}$. The values of these quantum numbers depend on whether the quark is left or right handed. The electric charge, weak isospin and weak hypercharge for quarks and leptons are summarized in Table 1.3.

In addition to weak hypercharge and weak isospin, quarks have an additional "charge" associated with the strong force. This charge is called color charge, and 


\begin{tabular}{|c|c|c|c|c|c|c|}
\hline \multicolumn{3}{|c|}{ Generation } & \multirow[t]{2}{*}{$T$} & \multirow[t]{2}{*}{$T^{3}$} & \multirow[t]{2}{*}{$Q$} & \multirow[t]{2}{*}{$Y$} \\
\hline 1 & 2 & 3 & & & & \\
\hline \multicolumn{7}{|c|}{ Leptons } \\
\hline$e$ & $\mu$ & $\tau)$ & & $1 / 2$ & -1 & \\
\hline$\nu_{e}$ & $\nu_{\mu}$ & $\left.\nu_{\tau}\right)_{L}$ & $1 / 2$ & $-1 / 2$ & 0 & -1 \\
\hline$e_{R}$ & $\mu_{R}$ & $\tau_{R}$ & 0 & 0 & -1 & -2 \\
\hline \multicolumn{7}{|c|}{ Quarks } \\
\hline$(d)$ & $s$ & $b)$ & $1 / 2$ & $-1 / 2$ & $-1 / 3$ & \\
\hline$u)$ & $c$ & $t)_{L}$ & $1 / 2$ & $+1 / 2$ & $+2 / 3$ & $1 / 3$ \\
\hline$d_{R}$ & $s_{R}$ & $b_{R}$ & 0 & 0 & $-1 / 3$ & $-2 / 3$ \\
\hline$u_{R}$ & $c_{R}$ & $t_{R}$ & 0 & 0 & $+2 / 3$ & $+4 / 3$ \\
\hline
\end{tabular}

Table 1.3: Summary of weak isospin, electric charge, and weak hypercharge for leptons and quarks.

there are three types of color charge: red $(R)$, green $(G)$, and blue $(B)$. Antiquarks carry charge antired $(\bar{R})$, antigreen $(\bar{G})$, and antiblue $(\bar{B})$.

Quarks are always found as bound states of hadrons - they are never free in nature. Because of this, the "bare" masses of the quarks can not be measured directly and must be inferred from their influence on hadrons. The exception to this is the top quark, which decays before becoming a bound state, thus allowing the measurement of the top mass from its decay products. The masses in Table 1.2 are the inferred bare masses.

There are two types of hadrons: baryons and mesons. Both baryons and mesons are "colorless particles". Baryons consists of three quarks or three antiquarks. They are fermions ( $\operatorname{spin}=n / 2$ where $n$ is an integral). For example, a proton consists of two up quarks and one down quark $(u u d)$. To satisfy the colorless criterion one quark is red, one blue, and one green.

Mesons consist of quark - antiquark pairs and have integral spin. For example, a $\pi^{+}$consists of an up and antidown quark $(u \bar{d})$ and has mass $\sim 139.6 \mathrm{GeV}$. The 
color of the down quark must be the anticolor of the up quark to make it colorless. The $\pi^{+}$has $J^{P}=0^{-}$where $J$ is the total angular momentum and $P$ is the parity. There exist "excited" states of the $u \bar{d}$ bound state, where the first bound state has $J^{P}=1^{-}$. This particle is called a $\rho$ and has mass $\sim 770 \mathrm{GeV}$. This property of "excited" states is not unique to the pion: there exist, for example, excited states of the uud bound state.

\subsubsection{Gauge Bosons and Forces}

Four forces govern the interactions between quarks and leptons: gravity, weak, electromagnetic, and strong forces. The Standard Model accounts for the latter three forces, and are the forces we will discuss in this section. The force of gravity is negligible compared to the strengths of the other forces.

Each force is mediated by a gauge boson, a spin 1 particle. The electromagnetic force carrier is the massless photon $(\gamma)$. The weak force has three massive mediators: $W^{ \pm}$and $Z^{\circ}$. The strong force is mediated by massless gluons. The $S U(3)_{C}$ symmetry group forms nine states: a "color octet" and a "color singlet". The 8 types of gluons that make up the octet are distinguished by their color charge: $r \bar{b}, r \bar{g}, b \bar{g}$, $b \bar{r}, g \bar{r}, g \bar{b}, \frac{1}{\sqrt{2}}(r \bar{r}-b \bar{b})$, and $\frac{1}{\sqrt{6}}(r \bar{r}+b \bar{b}-2 g \bar{g})$. The color singlet, $\frac{1}{\sqrt{3}}(r \bar{r}+b \bar{b}+g \bar{g})$, is "colorless" and does not exist in nature, else gluons would be found as free particles and they are not [1]. A "graviton" is expected to be the force carrier for gravity. Table 1.4 summarizes the properties of the gauge bosons associated with the weak, electromagnetic, and strong forces.

Quarks interact via the electromagnetic, weak and strong forces. The charged leptons (e, $\mu$, and $\tau$ ) interact via electromagnetic and weak interactions while neutrinos experience only the weak interaction. 


\begin{tabular}{|l|c|c|c|c|}
\hline & $\gamma$ & $W^{+}$ & $Z^{\circ}$ & $g$ \\
\hline \hline Force & $\begin{array}{c}\text { Neutral } \\
\text { EM }\end{array}$ & $\begin{array}{c}\text { Charged } \\
\text { Electroweak }\end{array}$ & $\begin{array}{c}\text { Neutral } \\
\text { Weak }\end{array}$ & Strong \\
\hline Mass [2] & $<2 \times 10^{-16}$ & $80.423 \pm 0.039$ & $91.1876 \pm 0.0021$ & 0 \\
\hline Lifetime [2] & stable & $\sim 3.11 \times 10^{-25}(s)$ & $\sim 2.64 \times 10^{-25}(s)$ & $\infty$ \\
\hline Spin & 1 & 1 & 1 & 1 \\
\hline Charge (e) & 0 & +1 & 0 & 0 \\
\hline Antiparticle & $\gamma$ & $W^{-}$ & $Z^{\circ}$ & $g$ \\
\hline
\end{tabular}

Table 1.4: Summary of gauge boson properties. The mass of the photon is given in $e V$, while the masses of the $W^{ \pm}$and $Z$ bosons are given in $G e V$.

We briefly discuss the theory of quantum chromodynamics, which describes the strong interaction, and the electroweak theory which combines the electromagnetic and weak forces.

\section{Quantum Chromodynamics}

Quantum Chromodynamics (QCD) is a "non-Abelian" gauge theory describing the strong interactions between quarks as asymptotically free and infrared confining.

The strong force mediators, gluons, carry color charge. This property renders the theory "non-Abelian" because the force carriers can interact with one another.

QCD interactions can be described by the potential [1]:

$$
V(r)=-\frac{4 \alpha_{s}}{3 r}+F_{\circ} r
$$

where $\alpha_{s}$ is the strong coupling constant and $r$ is the distance between the quarkantiquark pair. As $r$ increases $(r \gtrsim 0.1 \mathrm{fm})$ the second term dominates and the potential increases, forcing the quarks to hadronize forming quark-antiquark pairs. This property of the strong force is referred to by several terms including infrared 
confining, color confinement, and quark confinement. As a result, quarks are never found free and alway found as bound states of colorless hadrons.

For small $r(r \lesssim 0.1 \mathrm{fm})$ the first term in Equation 1.2 dominates. The strong coupling constant, $\alpha_{s}$, is not really a constant. Rather, it is a "running" coupling constant which depends on the distance between the quarks. If we consider a quark being probed, the distance between the probe and the quark corresponds to the probe energy: increases in probe energies correspond to decreases in distance. Then, $\alpha_{s}$ can be written in terms of the energy of the probe momentum transfer [1]:

$$
\alpha_{s}(Q)=\frac{12 \pi}{\left(11 n_{c}-2 n_{f}\right) \ln \left(|Q|^{2} / \Lambda_{Q C D}^{2}\right)}
$$

where $Q$ is the momentum transfer, $n_{c}$ is the number of color charges (3), $n_{f}$ is the number of quark flavors (6), and $\Lambda_{Q C D}$ is a scale factor. This form of the strong coupling constant shows that as the momentum transfer increases, corresponding to a decrease in distance, the interaction strength between quarks decreases. As $Q \rightarrow \infty, \alpha_{s} \rightarrow 0$ : this property is called asymptotic freedom. $\Lambda_{Q C D}$ provides a scale for the energy boundary between asymptotically free quarks and bound states of quarks.

\section{Electroweak Model}

The Glashow-Weinberg-Salam (GWS) electroweak model unifies the abelian electromagnetic $U(1)_{Q}$ gauge theory with the non-abelian $S U(2)_{L}$ weak isospin group to form the $S U(2)_{L} \times U(1)_{Y}$ electroweak group. $U(1)_{Q}$ represents the electromagnetic symmetry group which is generated by the electric charge Q. The $S U(2)_{L}$ weak isospin group acts only on left-handed particles, hence the $L$ subscript. $U(1)_{Y}$ represents the weak hypercharge group which couples to both left and right-handed 
particles.

According to the electroweak model, three weak isospin currents $\left(J_{\mu}^{i}\right)$ couple to an isotriplet of massive vector gauge fields $\left(W_{\mu}^{i}\right)$ with strength $g$, while the weak hypercharge current $\left(j_{\mu}^{Y}\right)$ couples to an isosinglet neutral gauge field $\left(B_{\mu}\right)$ with strength $g^{\prime} / 2$ such that the electroweak interaction can be written as [6]:

$$
-i g\left(J^{i}\right)^{\mu} W_{\mu}^{i}-i \frac{g^{\prime}}{2}\left(j^{Y}\right)^{\mu} B_{\mu}
$$

Then, the physical fields can be written in terms of $W_{\mu}^{i}$ and $B_{\mu}$ :

$$
W_{\mu}^{ \pm}=\sqrt{\frac{1}{2}}\left(W_{\mu}^{1} \mp i W_{\mu}^{2}\right)
$$

and:

$$
\begin{gathered}
A_{\mu}=B_{\mu} \cos \theta_{w}+W_{\mu}^{3} \sin \theta_{w} \\
Z_{\mu}=-B_{\mu} \sin \theta_{w}+W_{\mu}^{3} \cos \theta_{w} .
\end{gathered}
$$

$W_{\mu}^{ \pm}$represent the physical charged massive $W$ bosons, $Z_{\mu}$ represents the physical neutral massive $Z$ boson, and $A_{\mu}$ represents the neutral massless photon field. $\theta_{w}$ is the called the weak mixing angle. Clearly, the physical photon and $Z$ fields are mixes of the neutral $W_{\mu}^{3}$ and $B_{\mu}$ fields.

The weak mixing angle $\left(\theta_{w}\right)$ can be related to the weak isospin and weak hypercharge couplings and to the electromagnetic coupling $(e)$ by:

$$
e=g \sin \theta_{w}=g^{\prime} \cos \theta_{w}
$$


Furthermore, the weak mixing angle relates the $W$ coupling $\left(g_{W}\right)$ and $Z$ coupling $\left(g_{Z}\right)$ to the electromagnetic coupling by:

$$
\begin{array}{r}
g_{W}=\frac{e}{\sin \theta_{w}} \\
g_{Z}=g_{W} \cos \theta_{w}=\frac{e}{\sin \theta_{w} \cos \theta_{w}}
\end{array}
$$

The coupling of charged quarks and leptons to the photon field is purely vector. However, quarks and leptons couple to the $W$ and $Z$ bosons via both vector and axial vector coupling forms. The vertex that couples quarks and leptons to the charged $W$ field is purely $(V-A)$ :

$$
\frac{-i g_{W}}{\sqrt{2}} \gamma^{\mu} \frac{\left(1-\gamma^{5}\right)}{2}
$$

where $\frac{\left(1-\gamma^{5}\right)}{2}$ is the projection operator for left-handed currents. The pure $(V-A)$ coupling of the charged current vertex is a reflection of $W^{ \pm}$bosons coupling only to left handed fermions.

Because both right and left-handed particles couple to the $Z$ field, the vertex factor for coupling quarks and leptons to the neutral $Z$ field is a mix of vector and axial forms:

$$
\frac{-i g_{Z}}{2} \gamma^{\mu}\left(c_{V}^{f}-c_{A}^{f} \gamma^{5}\right)
$$

where $c_{V}^{f}$ and $c_{A}^{f}$ are the neutral vector and axial couplings, respectively. These couplings are specific to the quarks and leptons involved in the process and are specified in Table 1.5 [6].

The Higgs mechanism was introduced to the Standard Model as a means to give the $W$ and $Z$ bosons mass while leaving the photon field massless. This proposed 


\begin{tabular}{|l|c|c|}
\hline $\mathrm{f}$ & $c_{v}^{f}$ & $c_{a}^{f}$ \\
\hline \hline$\nu_{e}, \nu_{\mu}, \nu_{\tau}$ & $\frac{1}{2}$ & $\frac{1}{2}$ \\
\hline$e^{-}, \mu^{-}, \tau^{-}$ & $-\frac{1}{2}+2 \sin ^{2} \theta_{w}$ & $-\frac{1}{2}$ \\
\hline$u, c, t$ & $\frac{1}{2}-\frac{4}{3} \sin ^{2} \theta_{w}$ & $\frac{1}{2}$ \\
\hline$d, s, b$ & $-\frac{1}{2}+\frac{2}{3} \sin ^{2} \theta_{w}$ & $-\frac{1}{2}$ \\
\hline
\end{tabular}

Table 1.5: Neutral vector and axial couplings to Standard Model fermions (quarks and leptons).

Higgs field governs the masses of all particles according to the strength with which they couple to it. The propagator of the Higgs field, the Higgs boson, has not been experimentally observed.

Via the Higgs mechanism and the vector and axial couplings of quarks and leptons to the $W$ and $Z$ bosons, the GWS model relates the $W$ and $Z$ boson masses to the weak mixing angle by:

$$
M_{W}=M_{Z} \cos \theta_{w}
$$

This is a leading order, tree level equation which at higher orders would need to account for radiative corrections.

\subsection{3 $Z+\gamma, Z+Z$, and $W+Z$ Production}

Three electroweak Standard Model processes are important to this analysis because they are backgrounds to the dielectron + photon search signature.

The first, and largest background, is $Z+\gamma^{1}$ production, where the $Z$ decays leptonically in the electron channel. Figure 1.1 show the leading order Feynman

\footnotetext{
${ }^{1}$ When we discuss $Z+\gamma$ production, we include Drell-Yan production and so are considering: $Z / \gamma^{*}+\gamma$ processes.
} 
diagrams for $Z+\gamma$ production. $Z+\gamma$ production can occur when a photon is radiated off an incoming quark, as illustrated in (a) and (b) of Figure 1.1. This is referred to as initial state radiation. Another production mechanism for $Z+\gamma$ events is through final state radiation, where an electron radiates off one of the outgoing electrons, as shown in Figure 1.1(c).

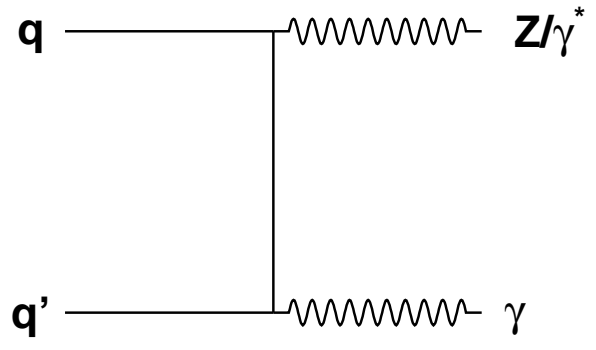

(a)

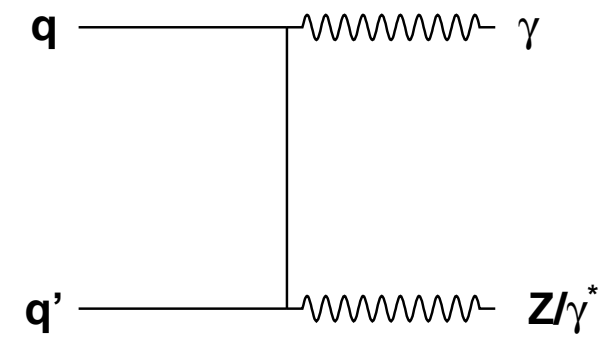

(b)

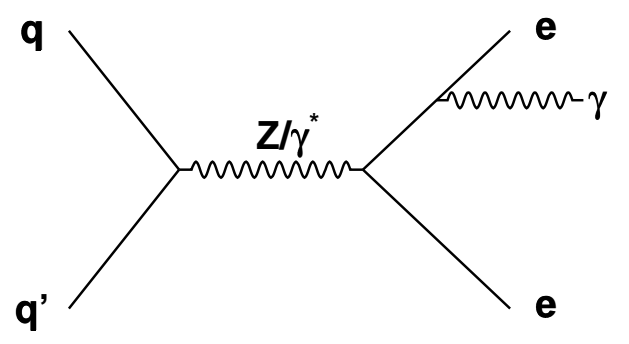

(c)

Figure 1.1: Tree level Feynman diagrams for $Z+\gamma$ production. Figures (a) and (b) are production via initial state radiation in the t-channel and u-channels, respectively. Figure (c) is inner bremsstrahlung production (also called final state radiation).

$W+Z$ events are a background source when both bosons decay in the electron channel: $W \rightarrow e \nu$ and $Z \rightarrow e e$. The result is three electrons in the final state. This becomes a background if one of the electrons travels through a portion of 
the detector that can not distinguish charged particles from neutral ones. More information about the detector is in Section 3.2. For now, we show in Figure 1.2 how $W+Z$ production occurs within the framework of the Standard Model.

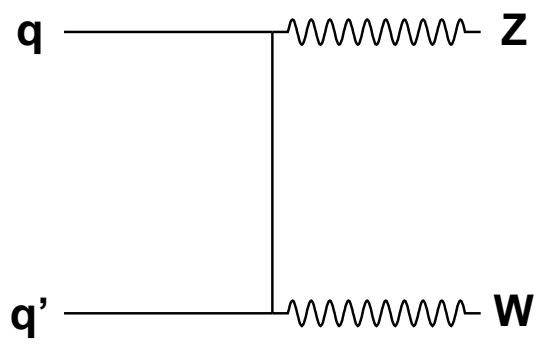

(a)

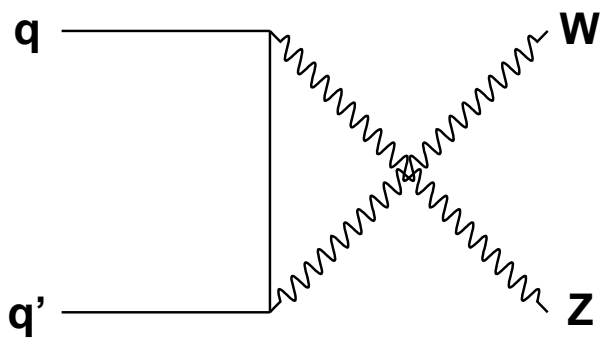

(b)

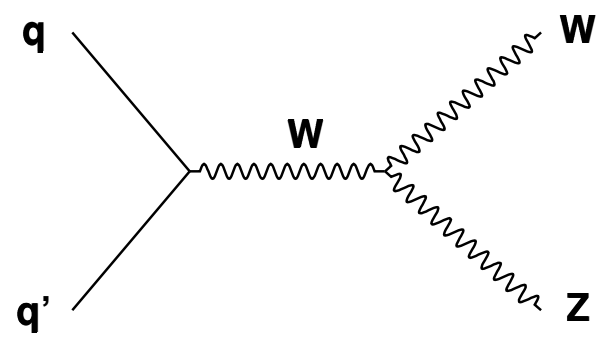

(c)

Figure 1.2: Tree level Feynman diagrams for $W+Z$ production. Figures (a) and (b) and (c) are t-channel, u-channel, and s-channel production, respectively.

Like $W+Z$ production, $Z+Z$ events can be a source of background when both $Z$ bosons decay in the electron channel, and only if one of the electrons is misidentified as a photon due to a lack of charge identification in the detector. Figure 1.2 illustrates how $Z+Z$ production occurs within the framework of the Standard Model. 


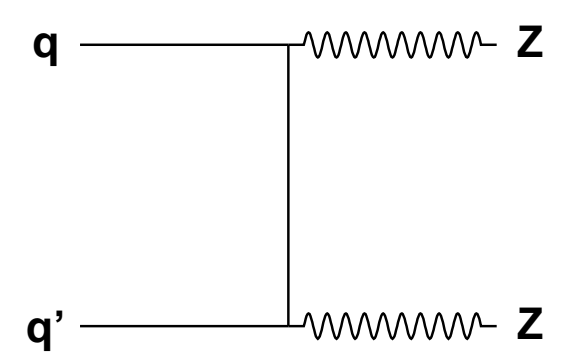

(a)

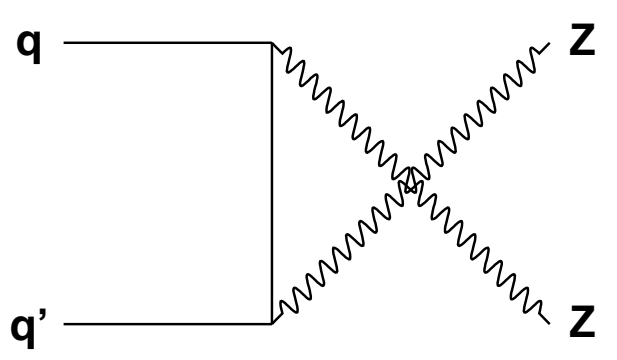

(b)

Figure 1.3: Tree level Feynman diagrams for $Z+Z$ production. Figures (a) and (b) are t-channel and u-channel production, respectively.

\subsubsection{Parton Distribution Functions}

At the Tevatron, protons and antiprotons are collided with a center of mass energy $(\sqrt{s})$ of $1.96 \mathrm{TeV}$. The interesting physics occurs in deep inelastic collisions, when the quarks and/or gluons in the protons interact with the anti-quarks and/or gluons in the antiprotons. However, the center of mass energy of a given quarkantiquark collision will not be $1.96 \mathrm{TeV}$ since the quarks carry only a fraction of the total proton energy. To understand the physics and draw good conclusions, one must have an understanding of the energy of the interacting particles. For this, an understanding of the structure of the proton is required.

We previously mentioned that a proton is a bound state of uud quarks. However, this is not a complete picture of the proton. Protons also have gluons interacting with the quarks and with each other. Gluon splitting spontaneously forms virtual quark-antiquark pairs within the proton. The quark-antiquark pairs are called sea quarks. The gluons constitute a significant portion of the proton momentum, $\sim$ $50 \%$. The net sum of sea quarks and antiquarks must be zero, resulting in a net 
quark content of uud. These uud quarks give the proton its quantum numbers and are called valence quarks.

Parton Distributions Functions (PDFs) describe the parton structure of the proton and the probability that a given parton carries fraction $x$ of the momentum. They are determined from experiments such as those using leptons as probes of the structure of the proton. Leptons will interact with quarks via electroweak forces, but not with gluons, at leading order. Measurements of the scattering cross section of the lepton-proton collisions provide a glimpse into the structure of the proton. The PDFs depend both on $x$ and the momentum transfer of the probe, $Q$. At low momentum transfer, the proton appears to be a point particle. As the momentum transfer increases, the structure of the proton and the sea of partons become more apparent.

The following sum rules must be observed to recover the valence quark structure of the proton:

$$
\begin{array}{r}
\int_{0}^{1}\left[u\left(x, Q^{2}\right)-\bar{u}\left(x, Q^{2}\right)\right] d x=2 \\
\int_{0}^{1}\left[d\left(x, Q^{2}\right)-\bar{d}\left(x, Q^{2}\right)\right] d x=1 \\
\int_{0}^{1}\left[S_{s, c, b}\left(x, Q^{2}\right)-\bar{S}_{s, c, b}\left(x, Q^{2}\right)\right] d x=0
\end{array}
$$

where $x$ is the fraction of momentum and $Q$ is the momentum transfer. Equation 1.14 means that the $u$ quarks minus the number of $\bar{u}$ quarks must be 2, the number of $u$ valence quarks. The $\bar{u}$ are all sea quarks, while the $u$ quarks are both sea and valence quarks. Likewise, Equation 1.15 requires the number of $d$ quarks minus the number $\bar{d}$ quarks equal the number of valence $d$ quarks, 1 . Equation 1.16 requires the sea of the other quark flavors $(s, c, b)$ to sum to zero. 
The total momentum of the proton is the sum of the momentum densities of each of the partons and gluons. The momentum density functions are given by the sum over all parton flavors and gluons of the integral of $x f\left(x, Q^{2}\right)$ over all possible values of $x$ :

$$
\sum_{f} \int_{0}^{1} x f\left(x, Q^{2}\right) d x=1
$$

Measurements from several experiments done over a range of $x$ and $Q^{2}$ have been combined to form sets of "global" parton distribution functions. In this analysis, we use the CTEQ5L ${ }^{2}$ as our primary PDFs. We compare the effect of various PDFs on event kinematics (Section 7.3.2) and generated cross-section (Section 11.4.2).

\section{$1.2 \quad$ Natural Units}

Throughout this thesis, including this chapter, we use a convenient choice of units that is often used in high energy physics: natural units. Natural units set the speed of light, $\mathrm{c}$, and Planck's constant divided by $2 \pi, \hbar$, to unity. Setting the speed of light equal to 1 makes length and time share the same dimensions. An effect of $\hbar$ being set to 1 is that the dimensions of mass and momentum are equivalent and equal to the dimensions of energy.

For example, in standard units, the dimensions of mass, $[\mathrm{M}]$, are $e V / c^{2}$, while in natural units $[\mathrm{M}]$ is simply $\mathrm{eV}$. Similarly, rather than express the dimensions of momentum, [p], as $e V / c$, they are expressed simply as $e V$.

\footnotetext{
${ }^{2}$ CTEQ stands for Coordinated Theoretical-Experiment Project on QCD
} 


\section{Chapter 2}

\section{Excited/Exotic Electrons}

The hierarchical structure of the Standard Model fermion families could be taken as an indication of quark and lepton substructure. Just as there exist "excited" states of mesons and baryons, so too one would expect excited states of quarks and leptons to be produced in high energy collisions if the quarks and leptons are composite. In fact, one might expect the observation of excited fermions to be the first indication of quark and lepton substructure. The proposed constituents of quarks and leptons are referred to as preons. In a compositeness model, a new, strong interaction binds the preons. This new interaction is often compared to the QCD strong force (Section 1.1.2) and would likely have similar properties: specifically non-abelian, asymptotic freedom, and infrared confining. These new, strong interactions may be expected to produce excited electrons strongly, with fairly large cross-sections.

\subsection{Excited Electron Models}

Two models for excited electron production are described: the contact interaction model and the gauge mediated model. The models consider excited electrons with spin and weak isospin equal to $\frac{1}{2}$. 


\subsubsection{Contact Interaction Model}

The contact interaction model describes an interaction in which the preons interact with one another. For collision energies below the compositeness scale $\Lambda$, these interactions may be described by an effective four-fermion Lagrangian [3]:

$$
\mathcal{L}=\frac{g^{2}}{2 \Lambda^{2}} j^{\mu} j_{\mu}
$$

where $j_{\mu}$ is the fermion current

$$
j_{\mu}=\eta_{L} \bar{f}_{L} \gamma_{\mu} f_{L}+\eta_{L}^{\prime} \bar{f}^{*}{ }_{L} \gamma_{\mu} f_{L}^{*}+\eta_{L}^{\prime \prime} \bar{f}^{*}{ }_{L} \gamma_{\mu} f_{L}+\text { H.c. }+(L \rightarrow R)
$$

and $f$ and $f^{*}$ represent the Standard Model and excited fermions respectively. In the calculations of [3], $g^{2}$ is set to $4 \pi$ and the $\eta$ factors for the left-handed currents are set to one, while the right-handed currents are neglected.

This analysis search is for $q+\bar{q} \rightarrow e^{*}+e$ production. Thus, substituting the fermion current, $j_{\mu}$, in Equation 2.1, the applicable effective Lagrangian is:

$$
\mathcal{L}=\frac{g^{2}}{2 \Lambda^{2}}\left(\left[\eta_{L} \bar{f}_{L} \gamma_{\mu} f_{L}\right]\left[\eta_{L}^{\prime \prime} \bar{f}^{*}{ }_{L} \gamma^{\mu} f_{L}\right]+H . C\right)
$$

The Feynman diagram in Figure 2.1 shows this.

The parton-level cross-section for contact interaction $e e^{*}$ production is given by [3]:

$$
\hat{\sigma}\left(q \bar{q} \rightarrow e e^{*}\right)=\frac{\pi}{6 \hat{s}}\left[\frac{\hat{s}}{\Lambda^{2}}\right]^{2}\left[1+\frac{v}{3}\right]\left[1-\frac{M_{e^{*}}^{2}}{\hat{s}}\right]^{2}\left[1+\frac{M_{e^{*}}^{2}}{\hat{s}}\right]
$$

where

$$
v=\frac{\hat{s}-M_{e^{*}}^{2}}{\hat{s}+M_{e^{*}}^{2}}
$$




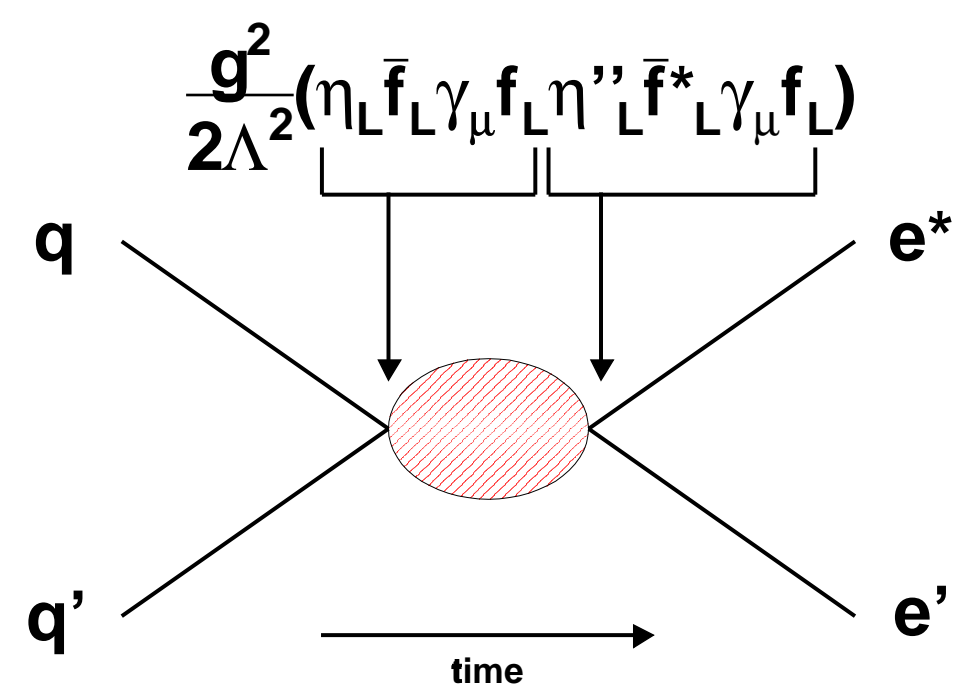

Figure 2.1: Feynman diagram of contact interaction $e^{*}$ production.

\subsubsection{Gauge Mediated Model}

Excited fermions can also be produced via gauge interactions. The model chosen in the literature couples left handed fermions to right handed excited fermions. These interactions may be described by the following Lagrangian [3] [4]:

$$
\mathcal{L}=\frac{1}{2 \Lambda} \bar{f}^{*} \sigma^{\mu \nu}\left[g_{s} f_{s} \frac{\lambda^{a}}{2} G_{\mu \nu}^{a}+g f \frac{\tau}{2} \cdot \mathrm{W}_{\mu \nu}+g^{\prime} f^{\prime} \frac{Y}{2} \mathrm{~B}_{\mu \nu}\right] \frac{1-\gamma_{5}}{2} f+\text { H.c. }
$$

The first term describes the coupling of excited fermions to the QCD gluon field, which would be relevant were we searching for excited quarks, but it is not applicable to excited electrons. Then, the effective Lagrangian becomes:

$$
\mathcal{L}=\frac{1}{2 \Lambda} \bar{f}^{*} \sigma^{\mu \nu}\left[g f \frac{\tau}{2} \cdot \mathrm{W}_{\mu \nu}+g^{\prime} f^{\prime} \frac{Y}{2} \mathrm{~B}_{\mu \nu}\right] \frac{1-\gamma_{5}}{2} f+\text { H.c. }
$$


Now, the first term in Equation 2.7 describes the coupling of excited leptons to the weak field and the second term defines the coupling to the electromagnetic field. Thus, $\mathrm{W}_{\mu \nu}$ and $\mathrm{B}_{\mu \nu}$ are the field strength tensors of the $\mathrm{SU}(2)$ and $\mathrm{U}(1)$ gauge fields. $\tau$ and $Y$ are the generators of the isospin and hypercharge gauge groups. $\Lambda$ is the compositeness scale, and $f$ and $f^{\prime}$ are phenomenological coupling constants. By convention, we set $f=f^{\prime}$ and typically study $e^{*}$ properties in terms of the ratio $f / \Lambda$. $Y$ is the weak hypercharge of the excited states, which is equal to -1 for left handed excited electrons. $g$ and $g^{\prime}$ are the electroweak coupling constants, $g=e / \sin \theta_{w}$ and $g^{\prime}=e / \cos \theta_{w}$, where $e$ is the electric charge and $\theta_{w}$ is the weak mixing angle, as discussed in the electroweak portion of Section 1.1.2.

It is useful to write Equation 2.7 in terms of the physical W, Z, and $\gamma$ fields, $\mathrm{W}_{\mu \nu}^{ \pm}, Z^{\mu \nu}$ and $A^{\mu \nu}$, respectively [4].

$$
\mathcal{L}=\sum_{V=W^{ \pm}, A, Z} \frac{e}{\Lambda} \bar{f}^{*} \sigma^{\mu \nu}\left(c_{V f^{*} f}-\gamma^{5} d_{V f^{*} f}\right) V_{\mu \nu} f
$$

$c_{V f^{*} f}$ and $d_{V f^{* f}}$ represent the couplings of the gauge fields to excited and ordinary electrons. These couplings have several experimental constraints. Measurements of the anomalous magnetic moment of electrons and muons $(g-2)$ imply $\left|c_{V f^{*} f}\right|=\left|d_{V f^{*} f}\right|$ and in this model $c_{V f^{*} f}=d_{V f^{*} f}[4]$. These couplings are defined as $[3][4][5]$ :

$$
\begin{array}{r}
c_{\gamma e^{*} e}=d_{\gamma e^{*} e}=f T_{3}+f^{\prime} \frac{Y}{2} \\
c_{Z e^{*} e}=d_{Z e^{*} e}=f T_{3} \cot \theta_{w}-f^{\prime} \frac{Y}{2} \tan \theta_{w} \\
c_{W^{ \pm} e^{*} \nu}=d_{W^{ \pm} e^{*} \nu}=\frac{f}{2 \sqrt{2} \sin \theta_{w}} .
\end{array}
$$


As usual, $T_{3}$ is the third component of the weak isospin, and $Y$ is the weak hypercharge of the excited states. For gauge mediated excited electron production and decay, $T_{3}=\frac{1}{2}$ and $Y=-1 . \theta_{w}$ is the Standard Model weak mixing angle. $f$ and $f^{\prime}$ are have been previously defined as $f=f^{\prime}=1$.

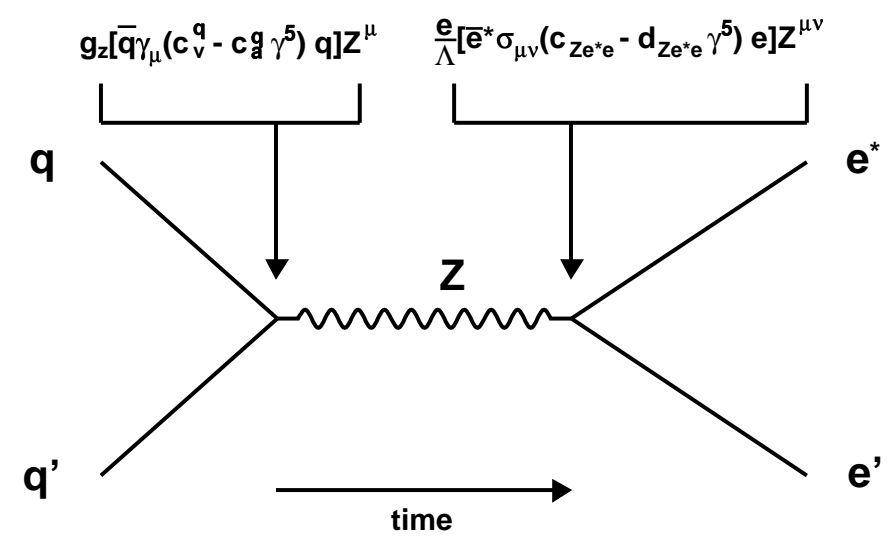

Figure 2.2: Feynman diagram of gauge mediated $e^{*}$ production via $Z$ exchange.

The differential cross-section for gauge mediated production has been calculated in the literature for the reaction $e \bar{e} \rightarrow Z / \gamma^{*} \rightarrow e^{*} e$ [4]. This is used to determine the differential cross-section for $q \bar{q} \rightarrow Z / \gamma^{*} \rightarrow e^{*} e$ by including a factor of three to account for the three color charges of quarks and replacing the lepton couplings with quark couplings. The result is:

$$
\frac{d \sigma}{d t}\left(q \bar{q} \rightarrow e^{*} e\right)=\frac{3 Q^{4}}{8 \pi s^{2} \Lambda^{2}}\left\{s\left(\left[m_{e^{*}}^{2}\left(s-m_{e^{*}}^{2}\right)+t u\right] A_{1}+m_{e^{*}}^{2}(t-u) A_{2}\right) D_{Z}(s) D_{Z}^{*}(s)\right\}
$$

$s, t$, and $u$ are the Mandelstam variables: total center of mass energy squared, momentum transfer squared and crossed momentum transfer squared, respectively. 
$Q$ is the quark charge. $D_{Z}(s)$ denotes the $\mathrm{Z}$ boson propagator:

$$
D_{Z}(s)=\left[s-m_{Z}^{2}\right]^{-1}
$$

The coefficients $A_{1}$ and $A_{2}$ in Equation 2.12 are functions of the coupling constants:

$$
\begin{aligned}
& A_{1}=\left(c_{v}^{q} c_{v}^{* q}+c_{a}^{q} c_{a}^{* q}\right) \cdot\left(c_{Z e^{*} e} c_{Z e^{*} e}^{*}+d_{Z e^{*} e} d_{Z e^{*} e}^{*}\right) \\
& A_{2}=\left(c_{v}^{q} c_{a}^{* q}+c_{a}^{q} c_{v}^{* q}\right) \cdot\left(c_{Z e^{*} e} d_{Z e^{*} e}^{*}+d_{Z e^{*} e} c_{Z e^{*} e}^{*}\right)
\end{aligned}
$$

$c_{Z e^{*} e}$ and $d_{Z e^{*} e}$ are the couplings defined in Equation $2.9 c_{v}^{q}$ and $c_{a}^{* q}$ represent the Standard Model neutral vector and axial couplings, the values of which are shown in Table 2.1 [6].

\begin{tabular}{|l|c|c|}
\hline $\mathrm{q}$ & $c_{v}^{q}$ & $c_{a}^{* q}$ \\
\hline \hline$u, c, t$ & $\frac{1}{2}-\frac{4}{3} \sin ^{2} \theta_{w}$ & $\frac{1}{2}$ \\
\hline$d, s, b$ & $-\frac{1}{2}+\frac{2}{3} \sin ^{2} \theta_{w}$ & $-\frac{1}{2}$ \\
\hline
\end{tabular}

Table 2.1: Neutral vector and axial couplings to Standard Model quarks.

\section{$2.2 e^{*}$ Decay Modes}

Excited electrons decay according to the Lagrangian in Equation 2.7 in three ways: $e^{*} \rightarrow e \gamma, e^{*} \rightarrow e Z$, and $e^{*} \rightarrow \nu W$. The decay mode chosen for this is analysis is $e^{*} \rightarrow e \gamma$, based upon the need for a clean, low background search signature and properties of the $e^{*}$ branching ratios. 


\subsection{1 $e^{*}$ Partial Widths}

The $e^{*}$ width is proportional to $M_{e^{*}}^{3} / \Lambda^{2}$. The partial decay widths can be written in terms of the couplings, $c_{V f^{*} f}$ and $d_{V f * f}$ (as in Equations 2.9-2.11), the fine structure constant $\left(\alpha=\frac{e^{2}}{4 \pi}\right)$, and the compositeness scale $(\Lambda)$. Equation 2.16 is the formula for the $f^{*} \rightarrow f V$ widths, where $f\left(f^{*}\right)$ represents a fermion (excited fermion) and $V$ is $\gamma, W^{ \pm}$, or $Z^{\circ}[3][5]$.

$$
\Gamma\left(f^{*} \rightarrow f V\right)=\frac{\alpha}{4} c_{V f * f}^{2} \frac{m_{f^{*}}^{3}}{\Lambda^{2}}\left(1-\frac{m_{V}^{2}}{m_{f^{*}}^{2}}\right)^{2}\left(1+\frac{m_{V}^{2}}{2 m_{f^{*}}^{2}}\right)
$$

\subsection{2 $e^{*} \rightarrow e \gamma$ Decay Channel}

The decay channel chosen for this analysis is $e^{*} \rightarrow e \gamma$, shown schematically in Figure 2.3. This channel provides a characteristic event signal that is not produced

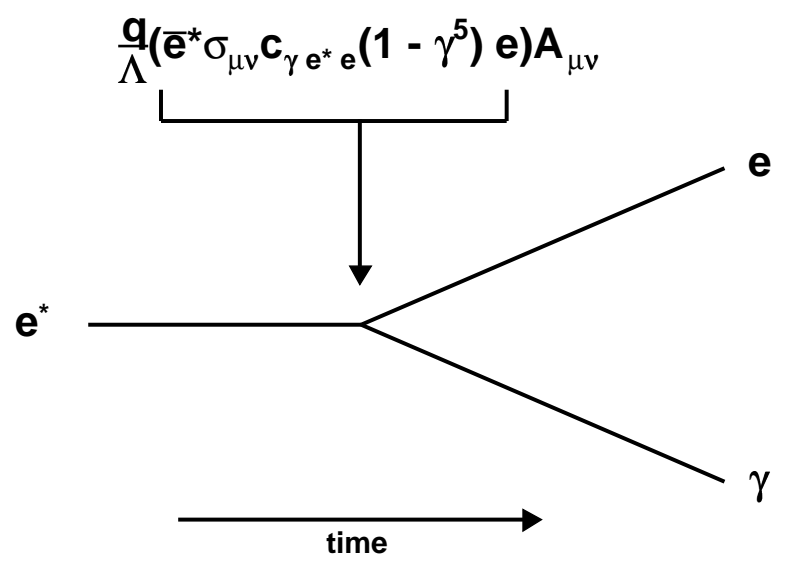

Figure 2.3: Feynman diagram of $e^{*} \rightarrow e \gamma$ decay

by many Standard Model processes. The only pure Standard Model process whose 
event signature is ee $\gamma$ is $Z / \gamma *+\gamma$ production:

$$
p+\bar{p} \rightarrow Z / \gamma^{*}+\gamma \rightarrow e+e+\gamma
$$

As we will see in Chapter 9, the expected background from $Z+\gamma$ production is small.

In the regions of the detector which do not have good tracking of charged particles, electrons can be misidentified as photons, or rather, the identity of an electromagnetic signal is ambiguous due to the absence of tracking information. In these cases, the Standard Model processes $W+Z$ and $Z+Z$ production:

$$
p+\bar{p} \rightarrow Z+Z \rightarrow e+e+e+e .
$$

and

$$
p+\bar{p} \rightarrow W+Z \rightarrow e+\nu_{e}+e+e .
$$

contribute to the background prediction.

The reason for choosing the $e^{*} \rightarrow e \gamma$ channel is more evident by the branching ratios. Figure 2.4 (a) shows the relative branching ratios (B.R.) for $e^{*} \rightarrow e \gamma$, $e^{*} \rightarrow \nu W$, and $e^{*} \rightarrow e Z$ as a function of $e^{*}$ mass, where the branching ratio is defined as:

$$
\text { Branching Ratio }=\frac{\Gamma\left(f^{*} \rightarrow f V\right)}{\sum_{V} \Gamma\left(f^{*} \rightarrow f V\right)}
$$

where $f^{*}$ is an excited electron and $f V$ are the three possible decay channels. As $M_{e^{*}}$ increases, the branching ratios become constant with 0.28 for $e^{*} \rightarrow e \gamma, 0.11$ for $e^{*} \rightarrow e Z$, and 0.61 for $e^{*} \rightarrow \nu W$.

The $Z$ in the decay mode $e^{*} \rightarrow e Z$ would need to decay leptonically to either 
electrons or muons in order to obtain a clean, low background signal eee or $e \mu \mu$. However, the branching ratios for $Z \rightarrow e e$ and $Z \rightarrow \mu \mu$ are $\sim 0.0337$ [2] for each channel. Figure 2.4(b) shows that the branching ratio for $e^{*} \rightarrow e Z \rightarrow$ eee and $e^{*} \rightarrow e Z \rightarrow e \mu \mu$ is negligible compared to the $e^{*} \rightarrow e \gamma$ channel. The branching ratios for $W \rightarrow e \nu_{e}$ and $W \rightarrow \mu \nu_{\mu}$ are $\sim 0.107$ [2] for each channel. Even if the $W$ in $e^{*} \rightarrow \nu W$ decayed leptonically, the signal would be $e \nu_{e} \nu_{e}$ or $\mu \nu_{\mu} \nu_{e}$, which are certainly not unique signals. Clearly, searching in the $e^{*} \rightarrow e \gamma$ channel is the best choice.

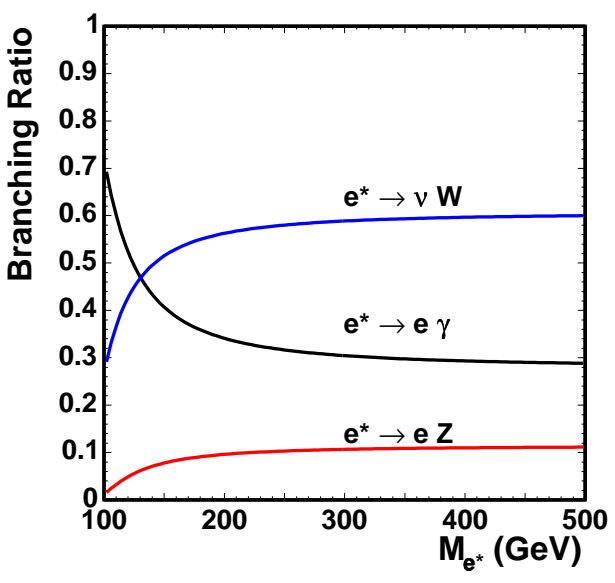

(a)

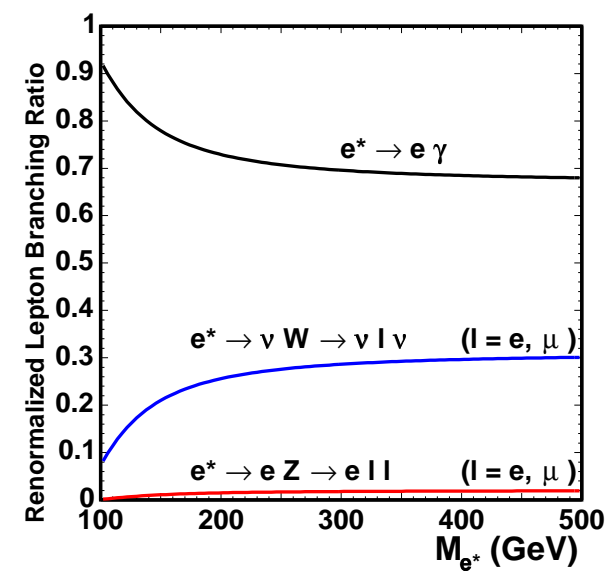

(b)

Figure 2.4: Figure (a) shows the branching ratios for each $e^{*}$ decay channel as a function of $e^{*}$ mass. Figure (b) shows the the renormalized branching ratios for each $e^{*}$ decay channel after forcing the $W$ and $Z$ to decay leptonically to electrons or muons.

\subsection{Monte Carlo Samples}

In general terms, Monte Carlo analysis is a numerical technique that uses sequences of random numbers to perform a simulation by calculating probabilities and related 
quantities. Given a set of input probability density functions which describe the system, the values of various quantities are computed using multiple trials of random numbers until there are enough values to estimate the properties of the quantities at the desired level of statistical precision. High energy physicists use several Monte Carlo programs to calculate cross-sections and generate events containing the kinematic information of various particle interactions.

\subsubsection{Contact Interaction Model}

PYTHIA 6.203 [7] is a Monte Carlo program that generates the kinematics of outgoing particles and calculates cross-sections for multi-particle processes at leading order. Excited electron production via contact interactions is implemented in PYTHIA. This is used to generate events for a series of excited electron mass values between $100 \mathrm{GeV}$ and $1000 \mathrm{GeV}$, with $\Lambda=M_{e^{*}}$, where the excited electron decays to $e \gamma$. The parton distribution function set CTEQ5L is used. Additionally, it is used to calculate the production cross-sections for many combinations of $M_{e^{*}}$ and $\Lambda$.

\subsubsection{Gauge Mediated Model}

The gauge mediated model is not implemented in PYTHIA 6.203. CompHEP [9] is a Monte Carlo program that evaluates the Feynman rules, calculates the matrix elements squared, generates events, and calculates cross-sections at leading order from a given input Lagrangian. There are a few processes already implemented in CompHEP, including the Standard Model processes. To implement the gauge mediated excited electron model in CompHEP, a program called LanHEP is used to write the Feynman rules in CompHEP format using the model Lagrangian as 
input [8].

Samples of excited electron events are generated for $e^{*}$ masses between 100 and $500 \mathrm{GeV}$ with the compositeness scale set equal to the mass. Again, the CTEQ5L parton distribution functions are used. These events are then run through PYTHIA which performs the fragmentation and hadronization processes to generate the final particle states. CompHEP is also used to calculate the cross-sections of several $M_{e^{*}}-\Lambda$ combinations.

\subsection{Excited Electron Properties}

\subsubsection{Invariant Mass Properties}

We look at the electron-photon invariant mass, which is the search channel, and the three-body (dielectron+photon) invariant mass spectra. For $e^{*}$ masses less than the compositeness scale $(\Lambda)$, the electron-photon invariant mass distribution is a narrow resonance peak. This is shown at parton level in Figure 2.5 for three choices $M_{e^{*}}$ and $\Lambda^{1}$. The areas under the curve are normalized to $202 p b^{-1}$ of data for the contact interaction model.

The three-body invariant mass spectra for the contact interaction model and gauge mediated model are shown in Figure 2.6 for three mass choices, where $M_{e^{*}}=$ $\Lambda$. The area under the curves is normalized to unity. The three-body invariant mass distributions are important when calculating higher order QCD corrections to the cross-section. This is discussed further in Section 2.4.3.

\footnotetext{
${ }^{1}$ Parton level means that Monte Carlo generated electron and photon momenta are used, ie. before running the detector simulation.
} 


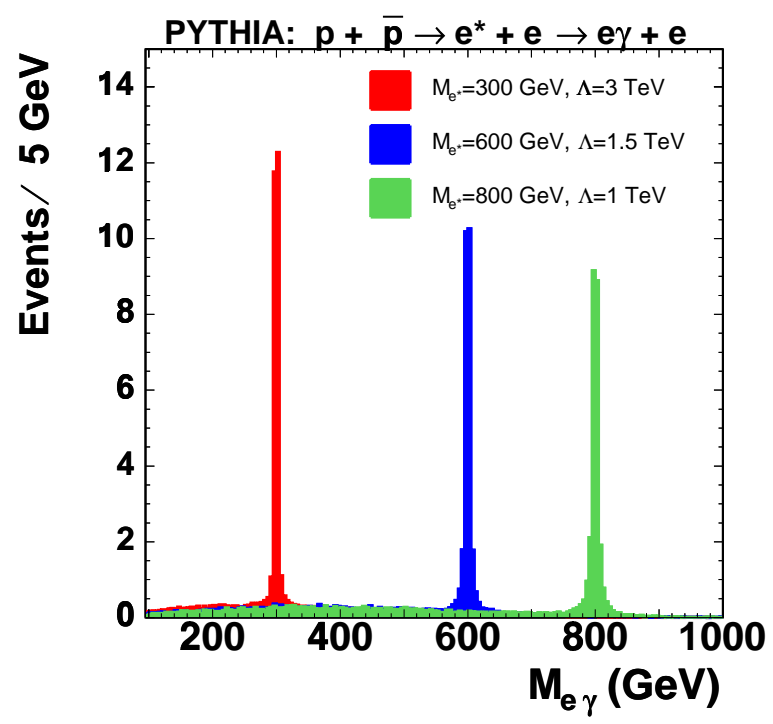

Figure 2.5: Parton level electron-photon invariant mass spectra for three choices of $M_{e^{*}}$ and $\Lambda$.

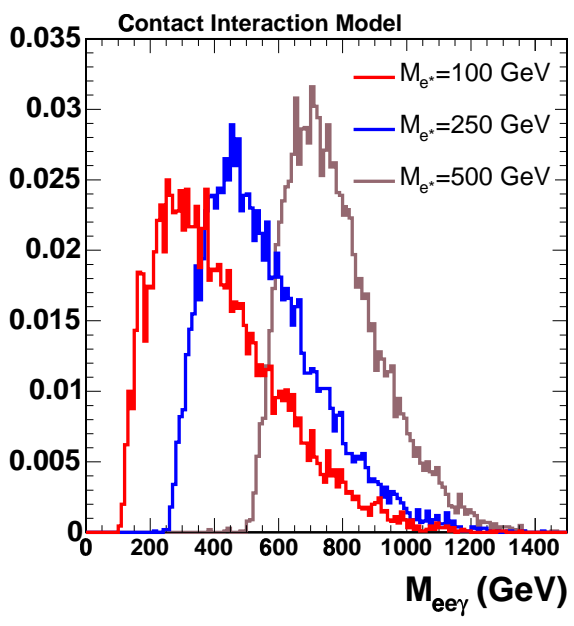

(a)

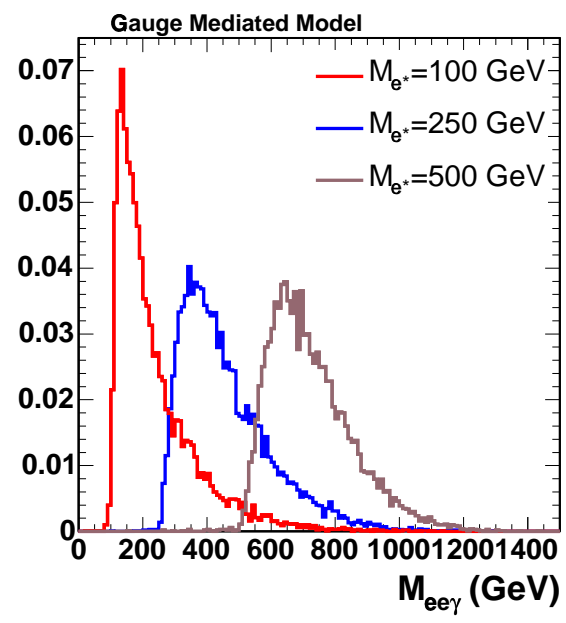

(b)

Figure 2.6: Three body invariant mass distributions for three mass choices for the contact interaction model (a) and the gauge mediated model (b), normalized to unit area. 


\subsubsection{Kinematic Properties}

In this section, we briefly investigate the kinematic properties of excited electrons at Monte Carlo generator level.

Figures $2.7-2.9$ show the transverse energy $\left(E_{T}\right)^{2}$ distributions of the final state particles for both $e^{*}$ models. Each figure has the distribution corresponding to $M_{e^{*}}=100,250,500$, and $700 \mathrm{GeV}$ for the contact interaction model and $M_{e^{*}}=100$, 250, and $500 \mathrm{GeV}$ for the gauge mediated model. Figures 2.7(a) and 2.7(b) are the $E_{T}$ distributions for the electron produced with the $e^{*}$ in the $q \bar{q} \rightarrow e^{*} e$ process. Figures 2.8 (a) and 2.8(b) are the distributions produced by the electron in the $e^{*}$ decay, $e^{*} \rightarrow e \gamma$, while Figures 2.9(a) and 2.9(b) are for the photon from the $e^{*}$ decay. Each plot is normalized to unit area.

These figures show that the electrons and photons have high $E_{T}$ with the $E_{T}$ distribution becoming harder as $e^{*}$ mass increases, as expected. Electrons produced in association with the excited electron are softer for the gauge mediated production than the contact interaction model for low $e^{*}$ mass. As the $e^{*}$ mass increases, this effect decreases.

Figures $2.10-2.12$ show the generated pseudorapidity ${ }^{3}$ distributions of the final state electrons and photon for both $e^{*}$ models. Each figure has the distribution corresponding to $M_{e^{*}}=100,250$, and $700 \mathrm{GeV}$ for the contact interaction model and $M_{e^{*}}=100$ and $250 \mathrm{GeV}$ for the gauge mediated model. Figures 2.10(a) and 2.10(b) are the pseudorapidity distributions for the electron produced with the $e^{*}$ in the $q \bar{q} \rightarrow e^{*} e$ process. Figures 2.11(a) and 2.11(b) are the distributions produced by the electron in the $e^{*}$ decay, $e^{*} \rightarrow e \gamma$, while Figures 2.12(a) and 2.12(b) are for the

\footnotetext{
${ }^{2}$ Transverse energy $\left(E_{T}\right)$ is the amount of energy directed perpendicular to the $Z$ axis.

${ }^{3}$ Pseudorapidity is defined in Section 3.2.1 and is denoted by the symbol $\eta$ in the plots.
} 


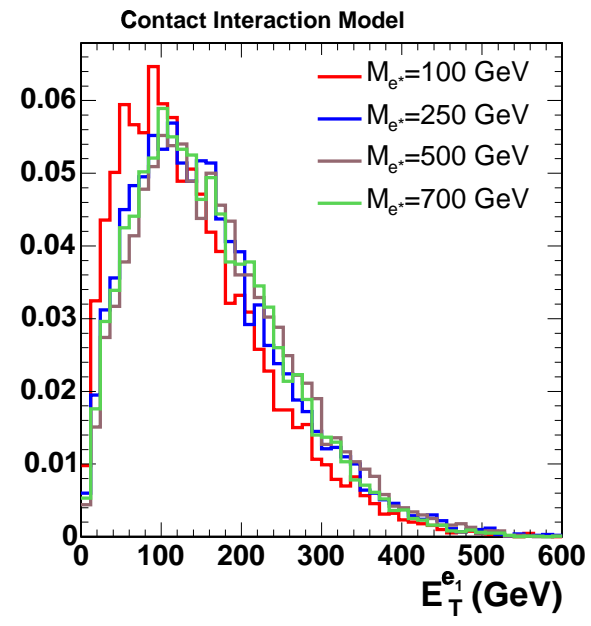

(a)

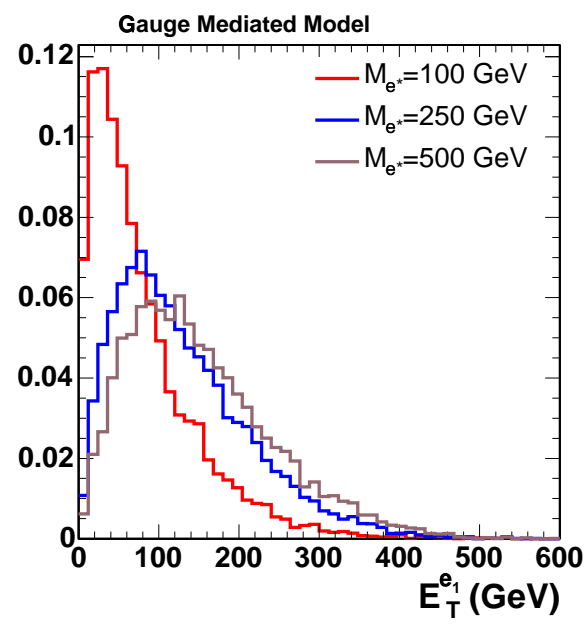

(b)

Figure 2.7: Parton level transverse energy distributions for the electron produced in association with the excited electron.

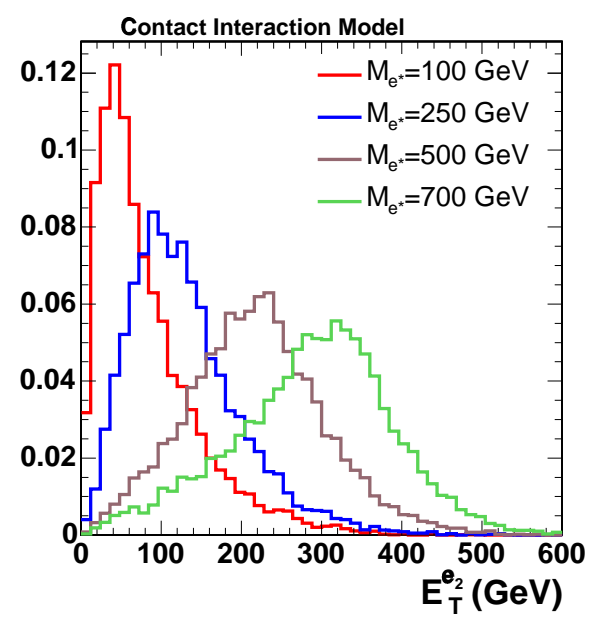

(a)

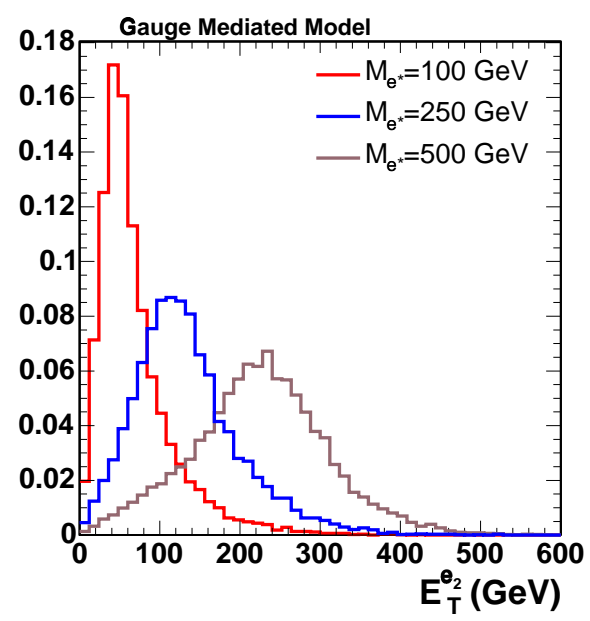

(b)

Figure 2.8: Parton level transverse energy distributions for the electron from the excited electron decay. 


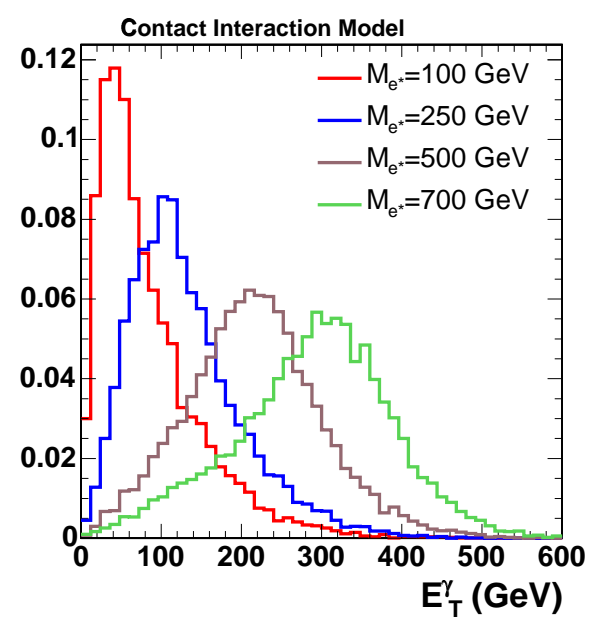

(a)

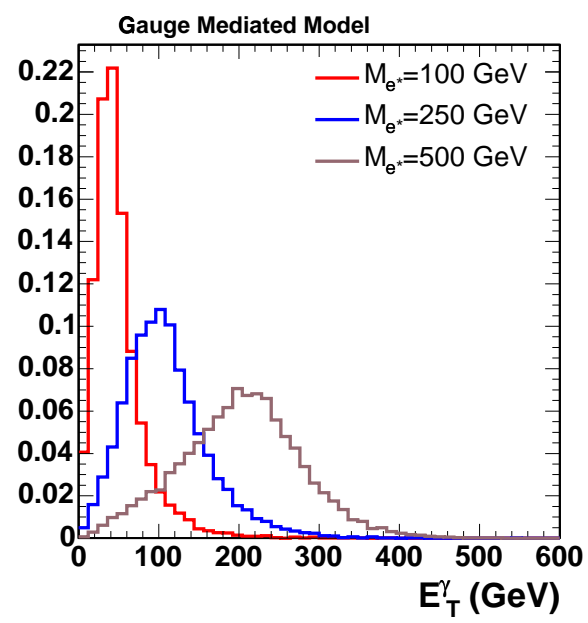

(b)

Figure 2.9: Parton level transverse energy distributions for the photon from the excited electron decay.

photon from the $e^{*}$ decay. Each plot is normalized to unit area.

These figures show that the absolute value of the pseudorapidity of the particles decreases with increasing $M_{e^{*}}$. This means that as $M_{e^{*}}$ increases, the particles are distributed in regions of the detector that have better coverage.

The dependence of the transverse energy and pseudorapidity distributions on $M_{e^{*}}$ indicate that the final state particles are easier to detect as $M_{e^{*}}$ increases.

\subsubsection{Excited Electron K-Factors}

Both Pythia and CompHEP calculate the leading order (LO), or tree-level, crosssections for excited electron production. Higher order QCD corrections due to the production of additional strongly interacting particles, quarks or gluons, need to be applied to the LO cross-sections. A program by Van Neerven [10] computes the Drell-Yan cross-section at LO, next-to-leading order (NLO), and next-to-next-to- 


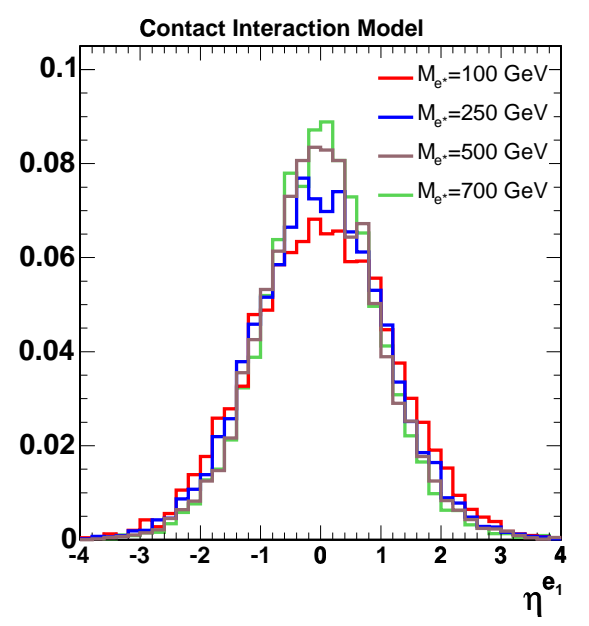

(a)

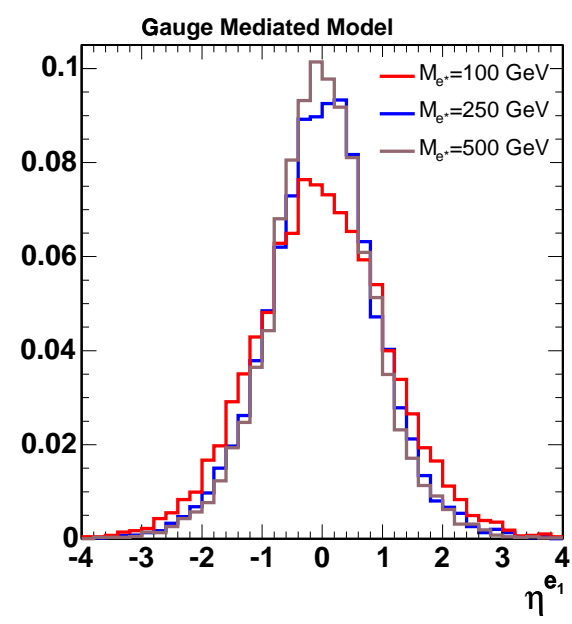

(b)

Figure 2.10: Parton level $\eta$ distributions for the electron produced in association with the excited electron.

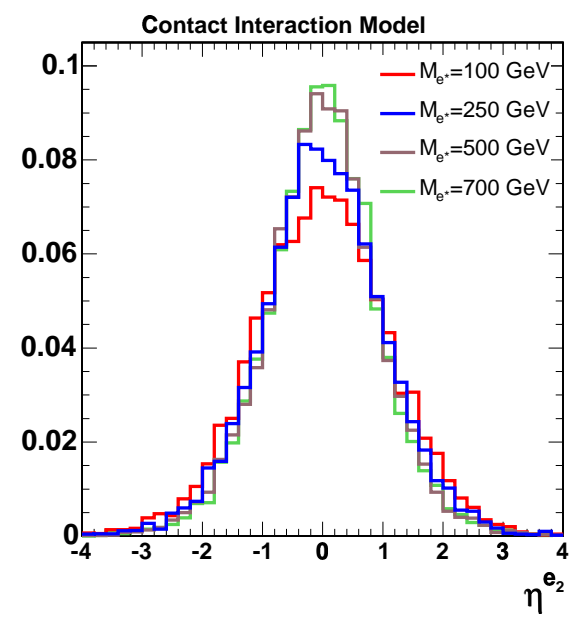

(a)

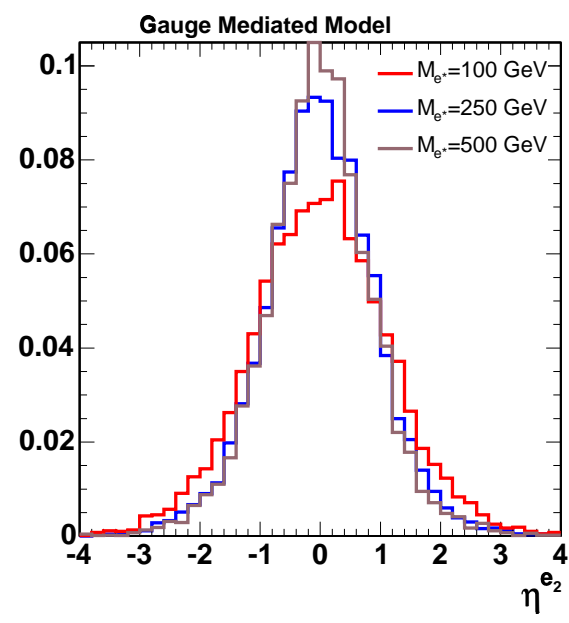

(b)

Figure 2.11: Parton level $\eta$ distributions for the electron from the excited electron decay. 


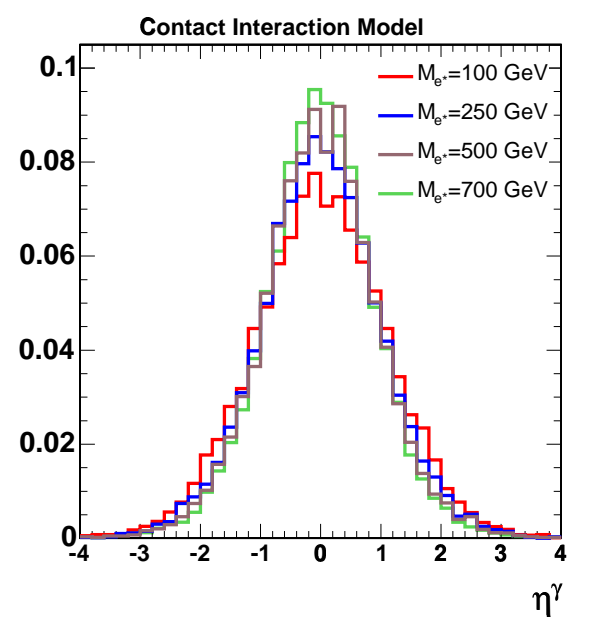

(a)

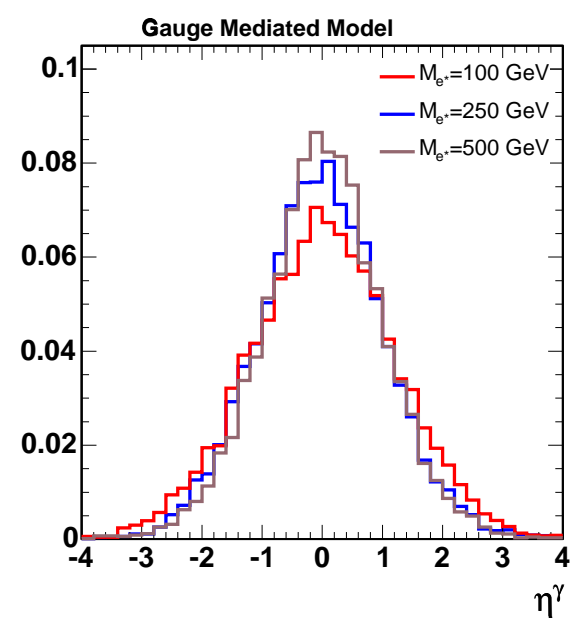

(b)

Figure 2.12: Parton level $\eta$ distributions for the photon from the excited electron decay.

leading order (NNLO) as a function of mass. They are calculated using the MRSDPDFs and the ratios of the NLO to LO cross-sections and the NNLO to LO crosssections determine NLO and NNLO K-factors, respectively. This method provides of means of determining mass dependent K-factors. These K-factors can be applied to any process with colorless final state particles according to the mass of the system produced by the collision. In the case of $e^{*}+e \rightarrow e \gamma+e$, it would be a function of the eqe three body invariant mass.

We find that for a particular set of kinematic cuts, the average $Z+\gamma$ three-body invariant mass is $128 \mathrm{GeV}$, corresponding to a Van Neerven NLO K-Factor of 1.22. We compare this Van Neerven K-Factor result to that given by U. Baur's ZGAMMA NLO cross-section calculation [11]. The Baur result is 1.33, differing from the Van Neervan result by $9 \%(=1.33 / 1.22)$. Thus, we correct the Van Neervan K-factors by applying a multiplicative scale factor of 1.09 . 
Table 2.4.3 shows the Van Neerven NLO and NNLO K-factors with the Baur correction factor and the uncertainty on the NNLO K-factor. The uncertainty is defined as the fractional difference between the NNLO and NLO calculations. This compensates for and assumes that any correction due to cross-section calculations at orders higher than the NNLO calculation is less than the difference between the NNLO and NLO results. Figure 2.13 [18] shows the NNLO K-factors as a function of system invariant mass. We use the shown fit, along with the three-body mass distributions of the generated $e^{*}$ processes (such as those shown in Figure 2.6), to determine the $\mathrm{K}$-factor at each $e^{*}$ mass point. The resulting $\mathrm{K}$-factors as a function of $M_{e^{*}}$, for $M_{e^{*}}=\Lambda$, are shown in Figure 2.14.

\begin{tabular}{|l|c|c|c|}
\hline Mass(GeV) & NLO K-factor & NNLO K-Factor & NNLO Uncertainty \\
\hline \hline 80 & 1.317 & 1.346 & 0.021 \\
200 & 1.344 & 1.395 & 0.036 \\
300 & 1.371 & 1.434 & 0.043 \\
400 & 1.404 & 1.479 & 0.051 \\
500 & 1.440 & 1.530 & 0.059 \\
600 & 1.481 & 1.589 & 0.068 \\
700 & 1.525 & 1.655 & 0.079 \\
800 & 1.572 & 1.728 & 0.090 \\
900 & 1.624 & 1.812 & 0.103 \\
950 & 1.652 & 1.857 & 0.110 \\
\hline
\end{tabular}

Table 2.2: NLO and NNLO K-Factors for 10 mass choices. The NNLO uncertainty is computed from the fractional difference between the NLO and NNLO values. 


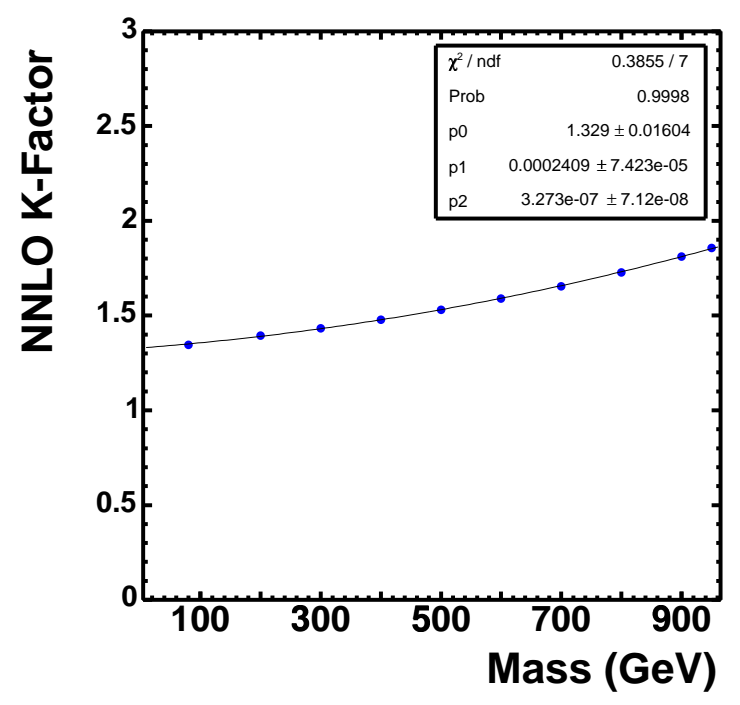

Figure 2.13: NNLO K-factors as a function of the system invariant mass.

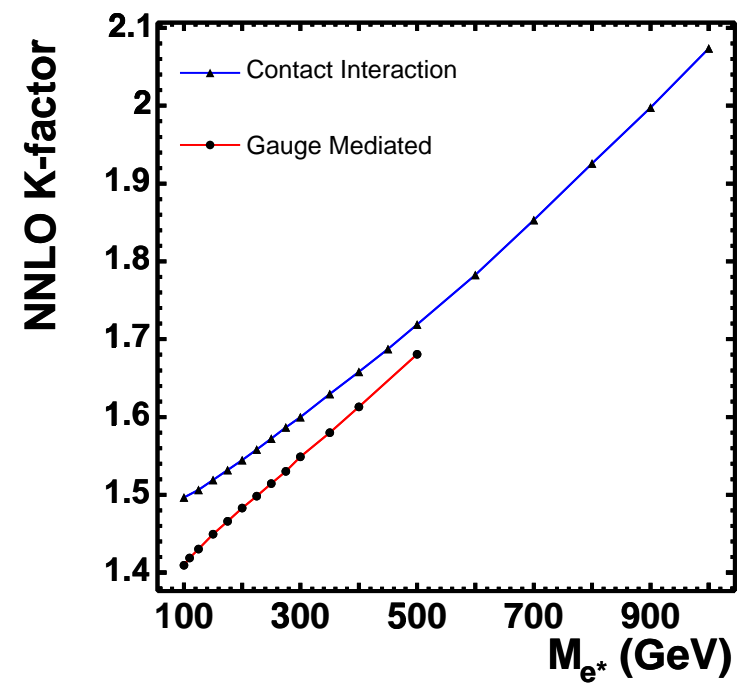

Figure 2.14: NNLO K-factors as a function of $e^{*}$ mass for the gauge mediated and contact interaction excited electron models. 


\section{Chapter 3}

\section{Fermilab Accelerator and CDF Detector}

To learn about the fundamental constituents of nature and their interactions, physicists use a series of apparatuses to accelerate and collide particles at high energies. This analysis uses data collected at Fermi National Accelerator Laboratory (Fermilab) in Batavia, Illinois. Fermilab is a proton-antiproton collider. In this chapter, the components of the accelerator and the detectors which are used to study the collisions are described.

\subsection{Accelerator}

A series of accelerators are used to accelerate protons $(p)$ and antiprotons $(\bar{p})$ to 980 $\mathrm{GeV}$ for a center of mass energy $(\sqrt{s})$ of $1.96 \mathrm{TeV}$. These accelerator components are shown in Figure 3.1.

A proton's journey to $980 \mathrm{GeV}$ begins by ionizing hydrogen gas to produce negative ions consisting of two electrons and a proton. In the Cockcroft-Walton, these ions are accelerated via a positive electrostatic voltage to $750 \mathrm{keV}$. Next, the $H^{-}$ions enter a $150 \mathrm{~m}$ long linear accelerator which boost their energy to $400 \mathrm{MeV}$ via oscillating electric fields. The ions subsequently pass through a carbon foil which 


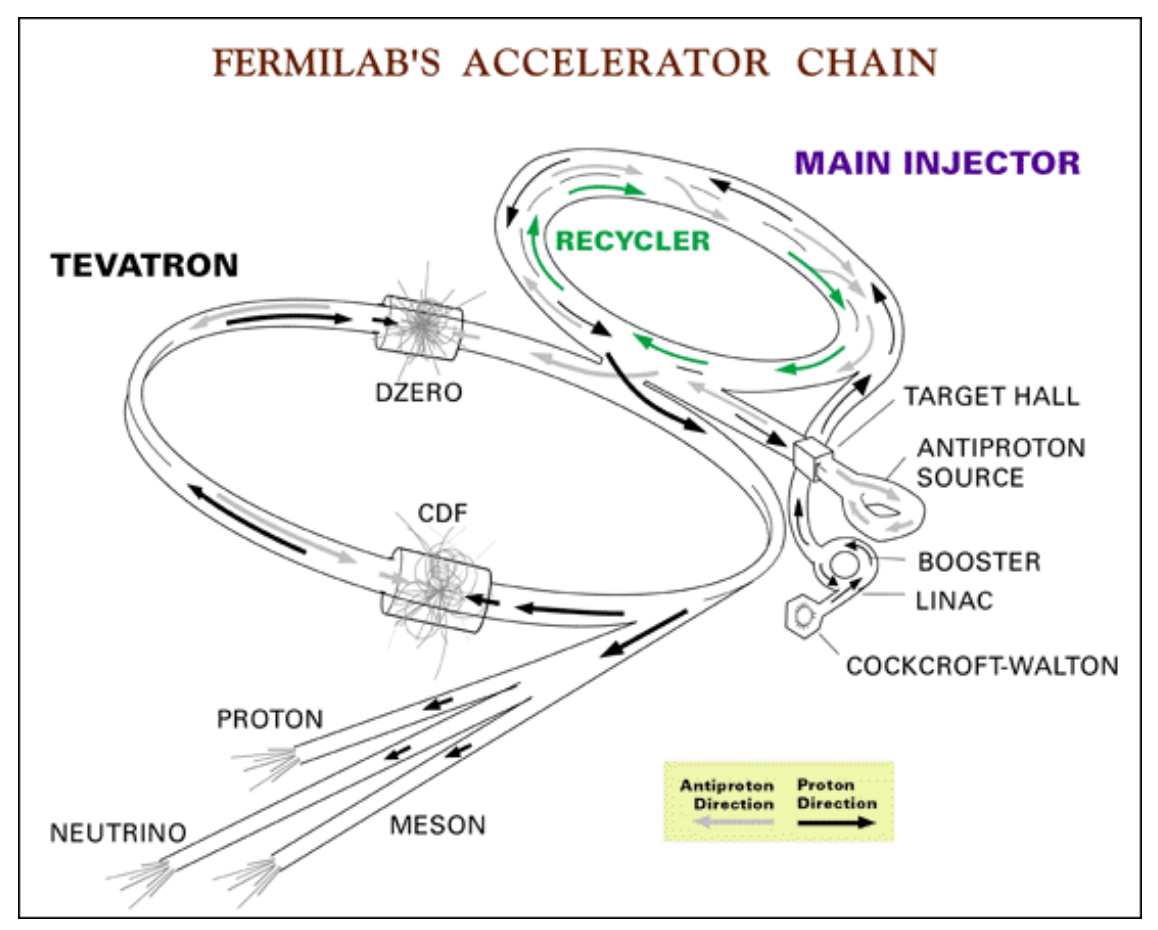

Figure 3.1: Schematic of accelerator components.

strips the electrons off the proton leaving only positively charged protons.

These protons enter the Booster, a synchrotron with a diameter of $150 \mathrm{~m}$. The Booster accelerates the protons to an energy of $8 \mathrm{GeV}$ by using magnetic fields to bend the trajectory of the particles and repeatedly applying the electric field during each revolution. The protons are collected into bunches consisting of $6 \cdot 10^{10}$ particles each.

The protons enter the Main Injector, a synchrotron that is $3 \mathrm{~km}$ in circumference, which accelerates the protons to energy $150 \mathrm{GeV}$. In addition to accelerating protons to $150 \mathrm{GeV}$, the Main Injector serves three other purposes. First, the Main Injector is used to send $120 \mathrm{GeV}$ protons to a nickel target, producing, among several secondary particles, antiprotons. These antiprotons are collected, focused, and stored. Second, the Main Injector accelerates the antiprotons to $150 \mathrm{GeV}$. Finally, 
it injects the protons and antiprotons into the Tevatron. In the same tunnel as the Main Injector is the Recycler. The Recycler is used to recycle antiprotons that are not used during a particular store instead of dumping the unused antiprotons. This saves time during the preparation of future stores and thus helps increase the total luminosity.

The final stage of acceleration of protons and antiprotons occurs in the Tevatron where they are accelerated in opposite directions to $980 \mathrm{GeV}$ each. The Tevatron is also a synchrotron with a circumference of about 4 miles. After 36 bunches of protons and 36 bunches of antiprotons are circulating in the Tevatron, they are focused using quadrupole magnets to collide. On average, collisions occur every 396 $n s$.

As shown in Figure 3.1, protons and antiprotons are collided at two interaction points: D0 and B0. B0's nominal interaction point is at the center of the Collider Detector at Fermilab (CDF). The CDF detector is described in the following sections.

\subsection{Collider Detector at Fermilab}

The Collider Detector at Fermilab (CDF) is a complex instrument used to study the properties of proton-antiproton collisions. It consists of many detector systems that measure various properties of the events. Many of the detector components and all of the data acquisition system were upgraded for Run II. These upgrades improved detector resolution and coverage region and allow the detector to handle the higher luminosity of Run II.

The CDF detector is a forward-backward and azimuthally symmetric detector. The geometric center of the detector is located at the nominal interaction point of 
the proton-antiproton collisions. The detector consists of several systems including tracking detectors, calorimeters and muon detectors. The tracking system, which is used to measure the properties of charged particles, surrounds the beam pipe. Outside the tracking detectors is the calorimetry system which is designed to measure the energies of certain types of particles. Beyond the calorimeters are drift chambers which are used for muon detection.

\subsubsection{Geometrical Coordinate System}

Figure 3.2 shows the geometric coordinate convention of the Tevatron and CDF. For the cylindrical coordinate system employed by CDF, the $+z$ direction is defined by the direction of the proton beam. As shown, the positive $y$ axis is upward while the positive $x$ axis is directed radially outward from the interaction point. The azimuthal angle $\phi$ is measured from the positive $x$ axis while the polar angle $\theta$ is measured from the positive $z$ axis. The $r$ coordinate of the CDF cylindrical coordinate system is defined by $r=z \cdot \cos \theta$.

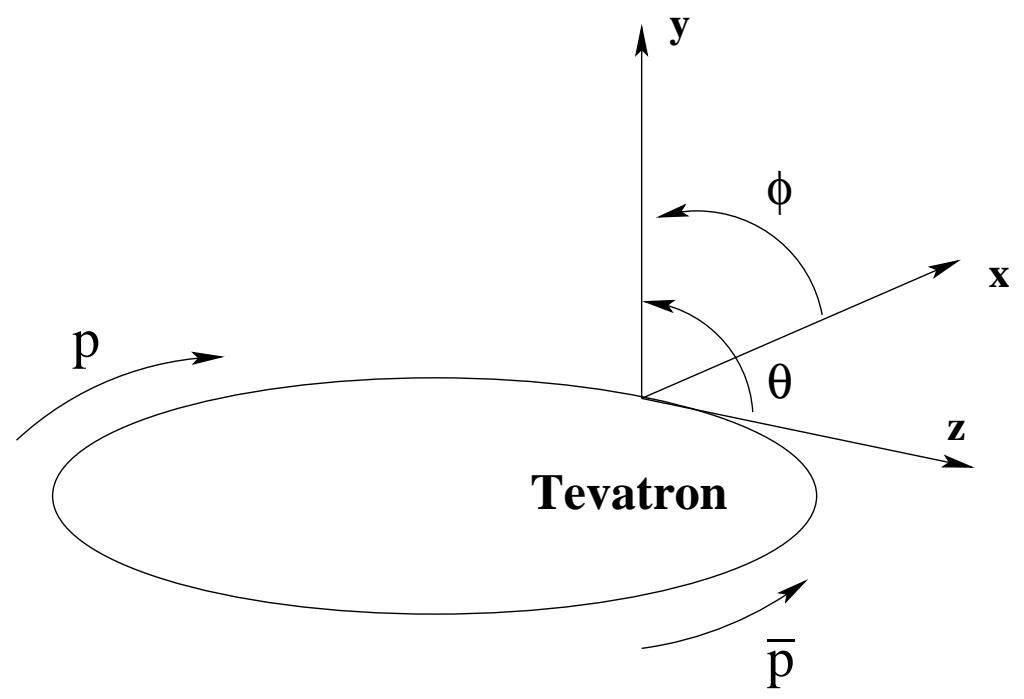

Figure 3.2: Tevatron/CDF coordinate system 
Instead of defining the trajectory of a particle by its polar angle $\theta$, the quantity pseudorapidity $(\eta)$ is used instead. Pseudorapidity is related to the quantity rapidity, $y$, which is defined as:

$$
y=\frac{1}{2} \ln \frac{E+p_{z}}{E-p_{z}}
$$

The pseudorapidity is a massless approximation of the rapidity $(E \approx p)$ and is defined by:

$$
\eta=-\log \left[\tan \frac{\theta}{2}\right]
$$

$\eta$ is symmetric about $\theta=90^{\circ}$ where $\eta=0$. Now, we distinguish between two definitions of $\eta$. The first is detector $\eta$, which is denoted as $\eta_{d}$, and defines the location a particle in the detector with respect to the center of the detector, $z=0$. We use $\eta$ to denote the event $\eta$ which is the actual pseudorapidity of the particle with respect to the interaction point of the event. $\eta$ and $\eta_{d}$ will be used throughout this analysis.

\subsubsection{Tracking System}

The tracking system is used to distinguish charged particles from neutral particles and to measure the position and momentum of charged particles in the detector. Figure 3.3 shows a longitudinal view of the tracking detectors. The innermost tracking detector is the Silicon Vertex Detector (SVX) which provides tracking information out to $\left|\eta_{d}\right|<2$. Surrounding the silicon tracking system is the central outer tracker (COT), a drift chamber, which provides tracking coverage for $\left|\eta_{d}\right|<1$. Finally, the entire tracking system is surrounded by a $1.4 \mathrm{~T}$ solenoid. 


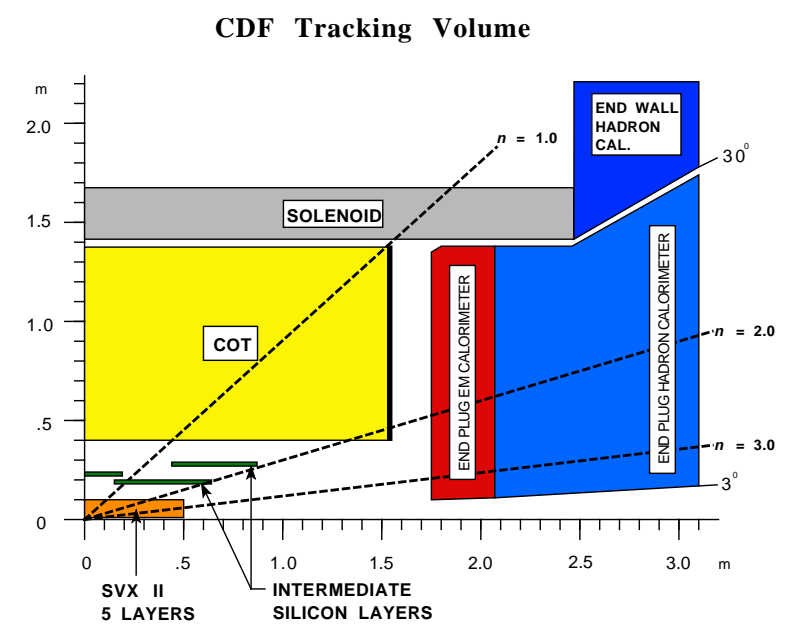

Figure 3.3: Longitudinal view of one quadrant of the CDF tracking system.

\section{Silicon Tracking}

The silicon tracking system consists of three subsystems designed for secondary 3D vertex measurements. The innermost detector, located immediately outside the beampipe is Layer 00 . This is a single sided layer of radiation tolerant silicon.

The SVX is located outside Layer 00 with inner radius $2.44 \mathrm{~cm}$ and outer radius $10.6 \mathrm{~cm}$. It consists of 3 barrels placed end to end. Each barrel is $29 \mathrm{~cm}$ in length for a total combined length of $87 \mathrm{~cm}$. Each barrel consists of 5 layers of double sided silicon and is segmented into 12 wedges in $\phi$. This design is shown in Figure 3.4.

While the SVX provides good impact parameter resolution for secondary vertex tagging, further information is necessary to match the silicon tracks to tracks in the central outer tracker, whose inner radius is located at $43.4 \mathrm{~cm}$. The intermediate silicon layers (ISL) serve this purpose in the central region by supplying a layer of silicon at a radius of $22 \mathrm{~cm}$ with $\left|\eta_{d}\right|<1$ coverage. Additionally, in the plug region, specifically $1<\left|\eta_{d}\right|<2$, the ISL consists of two more silicon layers at radii $20 \mathrm{~cm}$ and $28 \mathrm{~cm}$. As shown in Figure 3.5(b), these layers extend to $|z|<65 \mathrm{~cm}$ and $|z|<87.5 \mathrm{~cm}$, respectively, and provide additional tracking coverage where the 


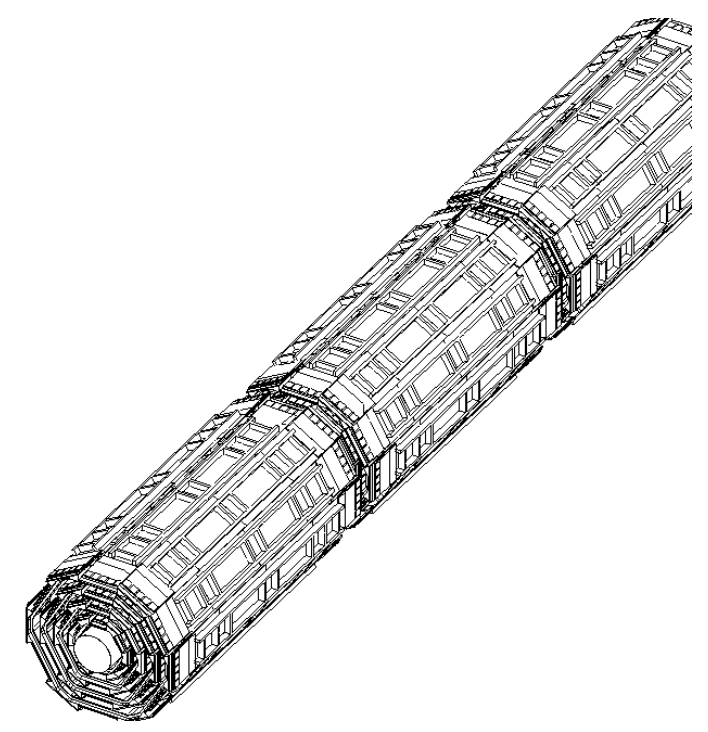

Figure 3.4: Schematic of SVX showing the 3 barrels placed end to end.

COT is incomplete. The geometry of the silicon system is shown in Figure 3.5, where Figure (a) is an end view and Figure (b) shows the ISL geometry in the $r-z$ view.

Further information on the SVX and ISL can be found in the CDF II Detector Technical Design Report [12]. This analysis does not depend on the silicon tracking system. Instead, the properties of central electrons are measured with the Central Outer Tracker (COT).

\section{Central Outer Tracker}

The Central Outer Tracker (COT) is a cylindrical open cell drift chamber used to detect and measure the three-dimensional paths of charged particles in the central region, $|\eta|<1.0$. As measured from the beam pipe, the inner radius of the COT is $43.4 \mathrm{~cm}$ and the outer radius of $132.3 \mathrm{~cm}$. The length of the COT is $310 \mathrm{~cm}$ along the beam direction. The cylindrical nature of the COT provides full coverage in $\phi$.

The chamber consists of 30,240 gold plated tungsten sense wires arranged in 


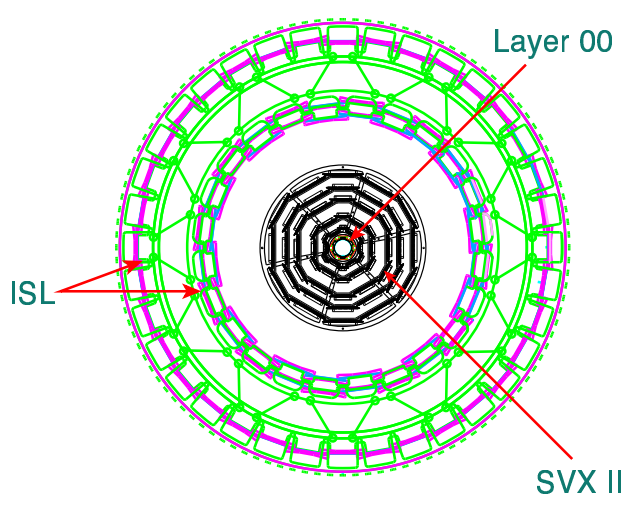

$64 \mathrm{~cm}$

(a)

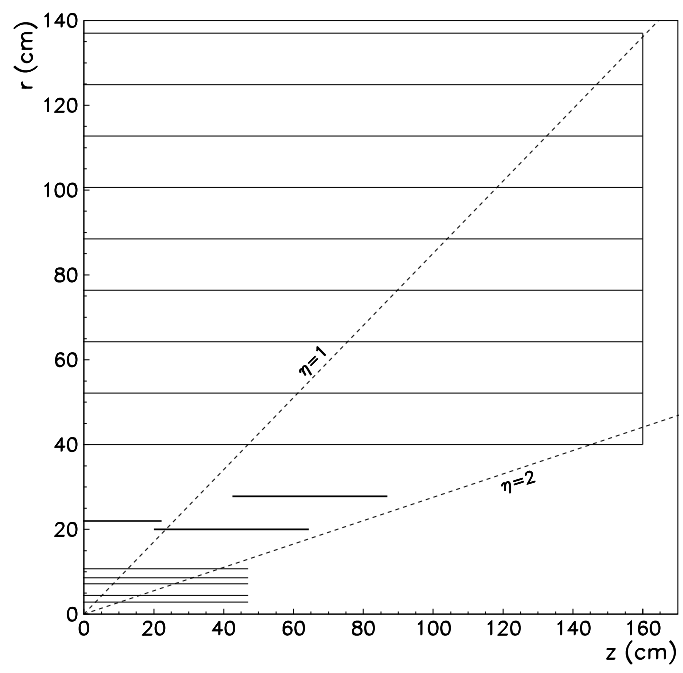

(b)

Figure 3.5: Figure (a) is an end view of silicon tracking system showing layer 00 + SVX + ISL making the $\phi$ segmentation apparent. The diameter of layer 00 is 2.2 $\mathrm{cm}$. Figure (b) shows an $r-Z$ view of the silicon detector layout. The ISL provides one additional silicon layer for matching SVX tracks to COT tracks and two layers for providing tracking coverage out to $\left|\eta_{d}\right|<2$. 
8 superlayers with 12 layers each [13]. The superlayers alternate between "axial" and "stereo" layers, where the innermost layer is a stereo layer. Axial layers, which provide tracking information in the $r-\phi$ plane, are arranged parallel to the Z-axis; stereo layers, which provide tracking information in the $r-z$ plane, are arranged at $\pm 2^{\circ}$ with respect to the beam line. Figure 3.6 depicts $1 / 6$ th of the east endplate, showing the axial and stereo superlayers. Each superlayer is divided into supercells in $\phi$, where each supercell consists of 12 wires. Figure 3.7 shows the wire layout for 3 cells of superlayer 2 .

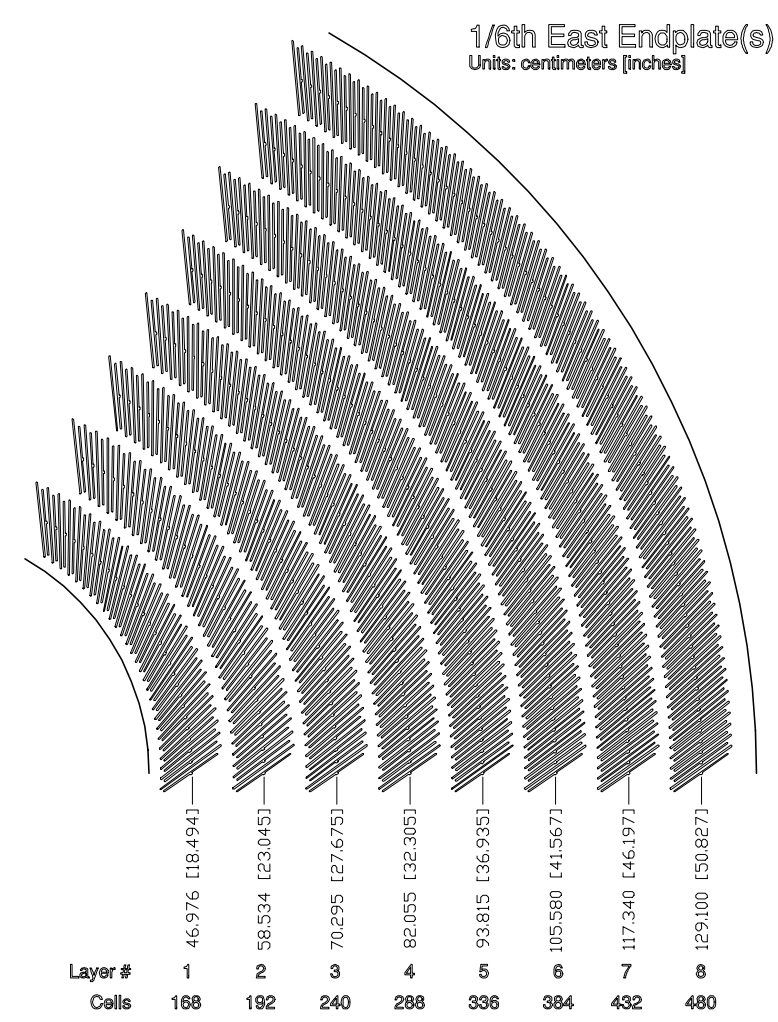

Figure 3.6: East endplate schematic of the 4 axial and 4 stereo superlayers. The odd numbered layers are stereo and the even numbered layers are axial. Shown are the radii of the center of the superlayers in $\mathrm{cm}[\mathrm{in}]$.

Charged particles passing through the chamber ionize the gas and the freed electrons drift to the sense wires. The gas used in the COT is a 50:50 Ar-Et + 
Isopropyl alcohol mixture which allows a maximum drift time of $\sim 177 \mathrm{~ns}$ [13]. The potential wires together with field sheets provide a uniform electric drift field of $1.9 \mathrm{kV} / \mathrm{cm}$. The $1.4 \mathrm{~T}$ solenoid surrounding the tracking chamber cause charged particles to travel in a helical trajectory through the detector. From this, the transverse momentum and charge of particles is measured from the track curvature. Because the crossed electric and magnetic fields cause the ionized electrons to drift with a Lorentz angle, the supercells are tilted $35^{\circ}$ with respect to the radial direction, as shown in Figure 3.7.

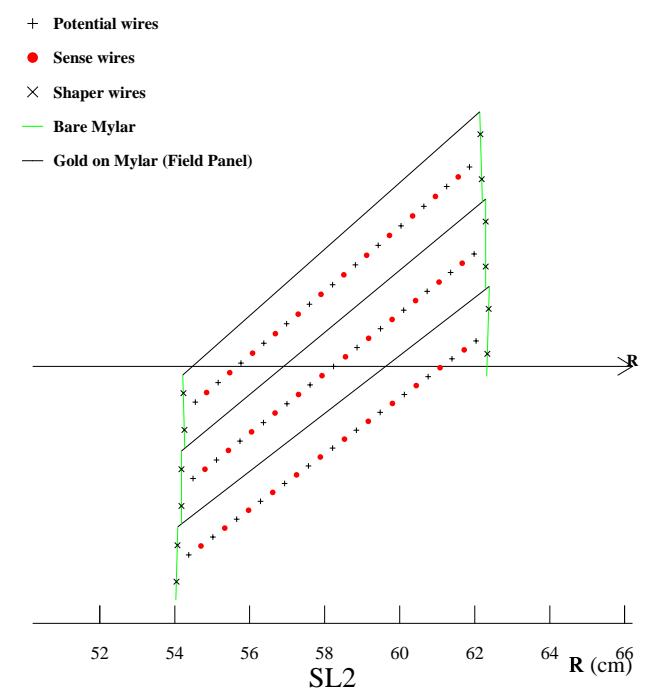

Figure 3.7: Schematic of cell layout for superlayer 2 showing the wire layout for 3 of the cells and the $35^{\circ}$ tilt with respect to $R$ to compensate for the Lorentz angle.

A central reconstructed track is fully defined by the following 5 helix parameters. The helix parameters are defined with respect to the track vertex, which is defined as the track position closest to the Z-axis.

- Curvature is defined as $(2 \cdot r)^{-1}$, where $\mathrm{r}$ is the radius of the track. The sign of the curvature is determined by the path of the track in the $\mathrm{x}-\mathrm{y}$ plane: 
clockwise tracks are denoted by negative curvature, while counter-clockwise tracks are positive. The track transverse momentum, $p_{T}$, is related to the curvature by:

$$
p_{T}=\frac{B q}{2 c}
$$

where $\mathrm{B}$ is the magnetic field, $\mathrm{q}$ is the charge of the particle and $\mathrm{c}$ is the track curvature. The momentum resolution, $\delta p_{T}$, is approximately $0.003 \cdot p_{T}^{2} / \mathrm{GeV}$. Thus, tracks with greater momentum are measured with poorer resolution.

- $d_{0}$, the impact parameter, is defined as the distance between the track vertex and the $z$-axis in the transverse direction. The sign of $d_{0}$ is defined as opposite the track angular momentum about the $z$-axis. The resolution on the impact parameter is approximately 300 microns.

- $z_{0}$ is the $Z$ position of the track vertex.

- $\cot \theta$ is the cotangent of the angle the track makes with the Z-axis. The resolution on the $\cot \theta$ is approximately 0.006 . This parameter is used for determining the event $\eta$ of electrons.

- $\phi_{0}$ is the direction of the track in the $\mathrm{x}$-y plane at the track vertex.

\subsubsection{Calorimetry System}

The calorimeters are sampling detectors that measure the amount of energy deposited by electrons, photons and jets. The calorimeters are positioned outside the tracking system. The total $\left|\eta_{d}\right|$ coverage ranges from 0 to 3.64. The system consists of central, plug, and end wall calorimeter regions. The central region spans $\left|\eta_{d}\right|<1$, and the plug, $1.1<\left|\eta_{d}\right|<3.64$. The end wall hadronic calorimeter covers a region 
between the central and plug region. This analysis utilizes the central and plug regions.

The calorimetry system consists of sandwiched layers of absorbing and sampling material. The absorbing material is a heavy metal which causes particles to shower and lose energy in the process. The sampling material consists of a scintillator which produces photons in response to the particle showers. The photons travel to light guides whose intensity is measured by phototubes. The intensity of the light produced is proportional to the number of particles $(N)$ produced in the shower and thus depends on the initial particle's energy. The uncertainty $(\delta N)$ on the number of particles produced is proportional to $\sqrt{N}$. Hence, since $E \propto N$, then the energy resolution $\delta E / E$ is proportional to $1 / \sqrt{N}$. The energy resolutions of the individual calorimeter regions are discussed in more detail later.

The calorimeter detectors are designed to distinguish energy deposited by electrons and photons (electromagnetic particles) from energy that is deposited by jets (hadronic particles). The electromagnetic calorimeters are located in front of the hadronic portions, with respect to the interaction point. Electrons and photons will generally shower before hadronic particles and thus lose most of their energy in the electromagnetic region of the detector. Conversely, hadronic particles such as pions and kaons, deposit minimal energy in the electromagnetic detector while showering primarily in the hadronic portion.

\section{Central Calorimeter}

The central calorimeter is a cylindrical detector located outside the COT spanning the $\eta_{d}$ region from -1.1 to 1.1. The detector consists of two barrels which meet in the center at $z=0, \eta_{d}=0$ : the west covering $-1.1<\eta_{d}<0$ and the east, $0<\eta_{d}<1.1$. 
Each barrel consists of 24 "wedges", for a total of 48 central calorimeter wedges. With the exception of gaps between the wedges and the "chimney", where the cryogenic connections to the solenoid are located, the central calorimeter provides full $2 \pi$ azimuthal coverage. A wedge spans $15^{\circ}$ in $\phi$ and is segmented into 10 "towers" in $\eta_{d}$. Thus one tower, the finest calorimeter segmentation, subtends $15^{\circ}$ in $\phi$ and approximately 0.11 in $\eta$. Each tower points back to the center of the detector as shown in Figure 3.8.

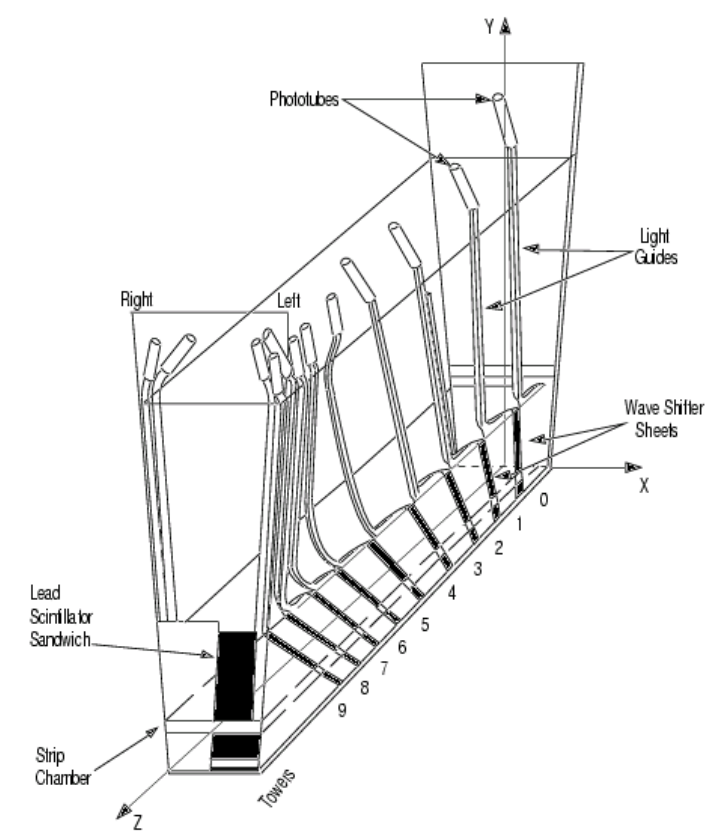

Figure 3.8: Schematic of one wedge of the central calorimeter. Each tower in the wedge is shown pointing back to the interaction point resulting in a projective geometry.

The absorbing material used in the Central ElectroMagnetic (CEM) calorimeter is lead. The layers of lead alternate with plastic scintillator, the sampling material. There are 30 layers of $3.2 \mathrm{~mm}$ lead sheets and 31 layers of $5 \mathrm{~mm}$ scintillator. The electromagnetic system has a longitudinal depth of 19 radiation lengths $\left(X_{\circ}\right)$. 
The fractional energy resolution of the CEM, $\delta E_{T} / E_{T}$, is $\sim 14 \% / \sqrt{E_{T}} \oplus \kappa$, where $E_{T}$ is the tranverse energy in $\mathrm{GeV} . \kappa$ is a non-energy dependent uncertainty due to sources such as mis-calibrations, tower-to-tower gain variations, and energy response differences within each tower. $\kappa$ is $\sim 1 \%$ in the central electromagnetic calorimeter.

Embedded near shower max, approximately 6 radiation lengths deep (between the eighth lead layer and ninth scintillator layer), is the Central Electromagnetic Shower max detector (CES). The CES measures the shape and location of particle showers to within $2 \mathrm{~mm}$. It is a proportional chamber with a drift gas mixture of 95\% argon and 5\% carbon dioxide. The CES consists of 64 anode wires aligned parallel to the beam and 128 cathode strips arranged perpendicular to the beam. The wires provide measurement of the "local X" position, which is defined as the distance from the center of the calorimeter in the $r-\phi$ direction. The strips provide measurement of the electron position in "local Z," along the direction of the beam. The transverse shape of the shower helps distinguish electrons and photons from hadronic particles. The local position of the tower is useful for associating electromagnetic particles with tracks, which are extrapolated to the CES from their helical parameters.

The Central HAdronic calorimeter ( $\mathrm{CHA})$ is contained in the same wedge as and directly behind the CEM wedges. The CHA is composed of 32 layers of 2.5 $\mathrm{cm}$ iron absorber interleaved with $1.0 \mathrm{~cm}$ thick layers of plastic scintillator. The CHA serves to promote the showering of hadronic particles. It provides a fractional energy resolution, $\delta E_{T} / E_{T}$, of $\sim 0.75 / \sqrt{E_{T}} \oplus \kappa$, where $\kappa$ is $\sim 5 \%$ and $E_{T}$ is in GeV. 


\section{Plug Electromagnetic and Hadronic Calorimeter}

The plug calorimeter of Run II is a completely new detector. Figure 3.9 shows a schematic of one quadrant of the plug detector system. As shown, the plug calorimeter spans the polar angle region from $37^{\circ}$ to $3^{\circ}\left(1.1<\left|\eta_{d}\right|<3.6\right)$. The electromagnetic calorimeter consists of 23 layers of $4.5 \mathrm{~mm}$ lead sheets interleaved with $4 \mathrm{~mm}$ polystyrene scintillator layers, corresponding to about $21 X_{\circ}$. The hadronic portion is 22 layers of 2 inch iron absorber alternating with $6 \mathrm{~mm}$ scintillator, for an approximate thickness of $7 \lambda_{\circ}$ (hadronic interaction lengths). The energy resolutions, $\delta E / E$, are proportional to the inverse of the square root of the energy plus a constant term, $\kappa$. The electromagnetic energy resolution is $\sim 0.16 / \sqrt{E} \oplus \kappa$, and the hadronic resolution is $\sim 0.80 / \sqrt{E} \oplus \kappa$. The energy is given in $\mathrm{GeV}$, and $\kappa$ is $\sim 1 \%$ and $\sim 5 \%$ in the electromagnetic and hadronic calorimeters, respectively.

Figure 3.9 portrays the geometry of the upper-east portion of the plug calorimeter. Figure 3.10 shows a schematic of the plug tower segmentation for a portion of the detector. These figures provide information regarding the physical geometry of the detector and the trigger tower geometry. In Figure 3.10, the $z$ axis, or beamline, is perpendicular to the page.

\subsubsection{Central Electromagnetic Pre-Radiator Chambers}

The Central electromagnetic Pre-Radiator detector (CPR) is a collection of multiwire proportional chambers that are located outside of the solenoid and in front of the electromagnetic calorimeter. The CPR can be used for improving the identification of electrons, distinguishing single prompt photons from meson decay, and improving jet resolutions. In this analysis, we use the CPR for distinguishing single prompt photons from those produced by the decay of mesons. The CPR is discussed 


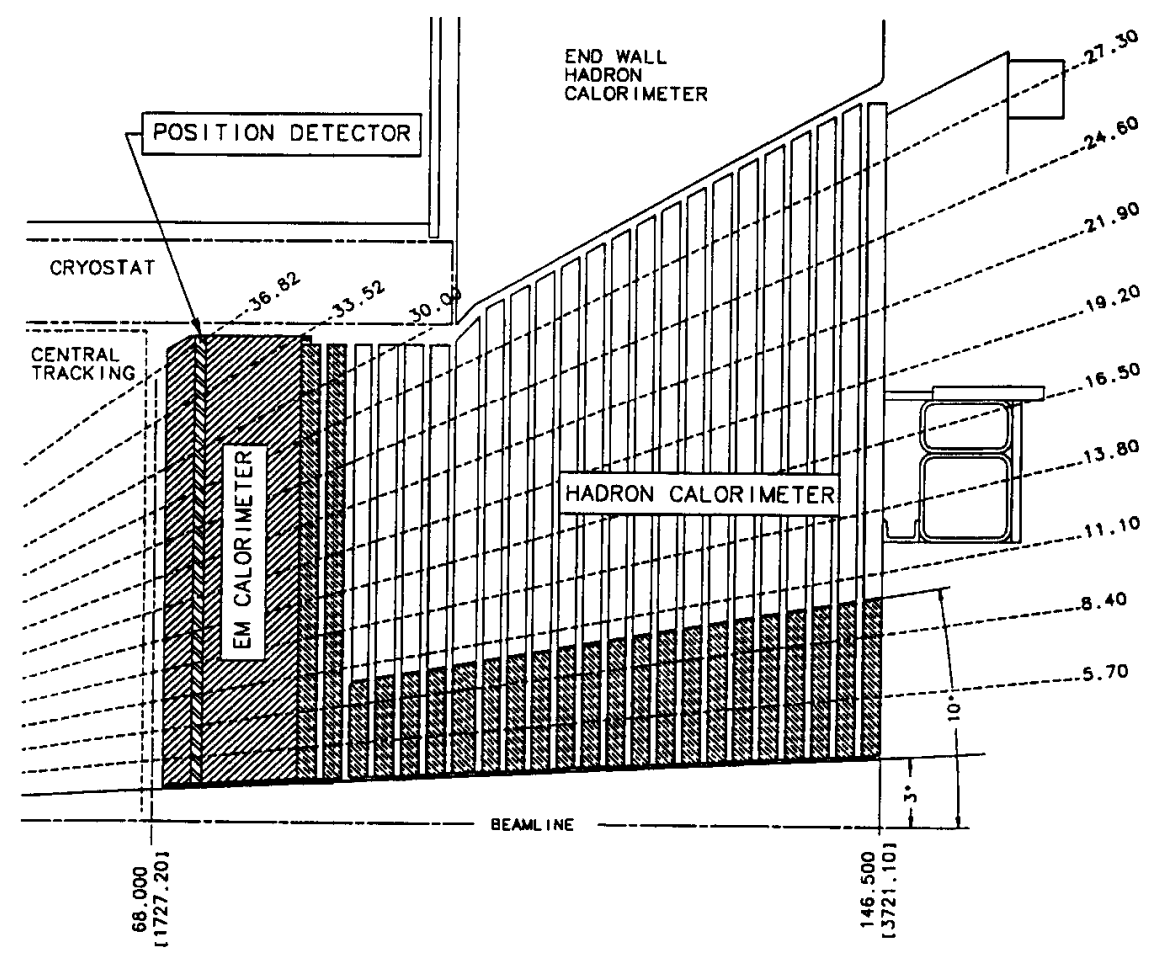

Figure 3.9: Schematic of one quadrant of plug detector system. 


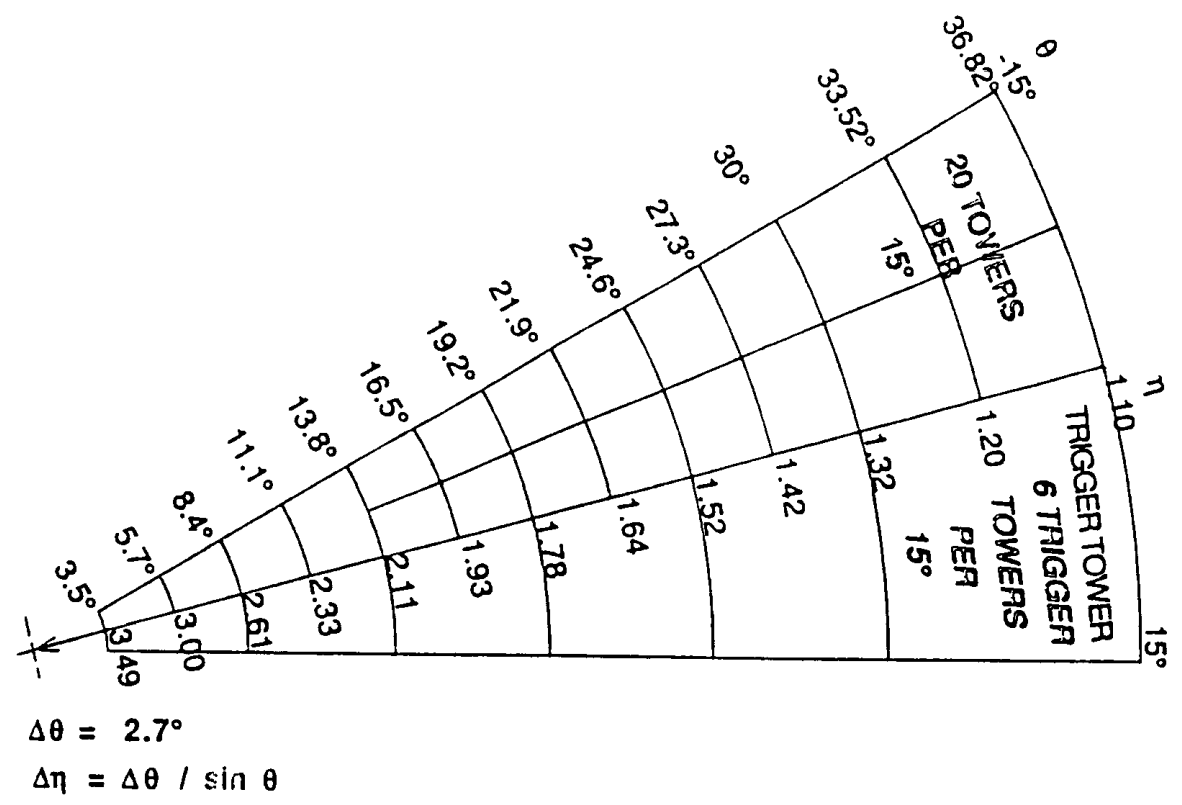

Figure 3.10: Plug tower geometry.

in more detail in Section 8.3.2.

\subsection{Trigger}

The rate at which $p \bar{p}$ collisions occur at the Tevatron is much higher than the rate at which events can be written to tape, and the majority of these collisions are soft collisions whose final state particle content is not interesting. The trigger system is designed to recognize events that are of interest to various physics topics and designate those events to be written to tape. The trigger has a three-level architecture. The goal is for each level to reduce the rate of events that pass to the next level with minimal deadtime. Each level is described in the following sections. In Section 4.1, the specific trigger requirements relevant to this analysis are summarized. 


\subsubsection{Level 1}

The first level in the three level trigger architecture is level- 1 which relies on custom designed hardware that identifies physics object candidates such as electrons, muons, and jets. The decision to pass an event to level-2 is determined by the identification of the object candidates and simple counting of said objects. The level-1 hardware identifies physics objects via three parallel synchronous processing streams which feed input to a single global decision unit. One stream finds calorimeter based objects (such as electrons, photons and jets), the second looks for muons, while the third, the eXtra Fast Tracker (XFT), locates tracks in the central tracking chamber. The information from each of these three parallel streams can be combined using simple AND and OR requirements for a total of 64 different possible triggers. The elements of the level-1 trigger are synchronized to a $132 \mathrm{~ns}$ clock, resulting in a level-1 trigger every $132 \mathrm{~ns}$.

\subsubsection{Level 2}

Events accepted by the level-1 trigger are processed by the level- 2 trigger which is composed of several asynchronous subsystems. These subsystems provide information to programmable level-2 Processors that determine whether any of the level-2 triggers are satisfied.

Figure 3.11 shows the trigger system block diagram. It shows that the level-1 trigger uses calorimeter, muon, and tracking information to make a decision. If an event passes level-1, level-2 uses the same information as level-1, but with better precision and also uses information from CES and SVX.

There are four level-2 buffers to which events that pass level-1 can be written. Each buffer can analyze one and only one event at a time. If all four buffers are 
in use, deadtime occurs. To keep the deadtime at an acceptable level, the time required for a level-2 decision needs to be approximately $80 \%$ of the time between level-1 accepts. To accomplish this, the level-2 trigger is pipelined in two stages that each take approximately $10 \mu \mathrm{s}$. The first stage is an event building stage which uses the level-1 event information at a higher level of precision. All the information about the event is loaded into the memory of the level-2 processors. Event building and the collection of information into the memory encompass the first stage of the pipeline. The event then moves to the second stage which examines whether the event passes any of the criteria required by a level-2 trigger. While an event is being analyzed in the second stage of the pipeline, information from the next event can be loaded into the level-2 memory (stage 1).

\subsubsection{Level 3}

The level-3 trigger uses the full detector resolutions and reconstructs events in a farm of linux computer processors. The level- 1 and level- 2 algorithms use predefined pieces of event information to make fast, yet crude, decisions on whether to pass an event. The level-3 trigger takes all the event information and fully reconstructs the event in the same way as it is done offline. At this level, the trigger determines whether the event passes criteria to be permanently stored on tape.

Further details about the triggers can be found in [12].

\subsection{Detector Simulation}

We need to study the expected properties of various physics processes such as $Z+\gamma$, $Z+Z, W+Z$, and $e^{*}$ production before looking at the data. In order to study such 


\section{RUN II TRIGGER SYSTEM}

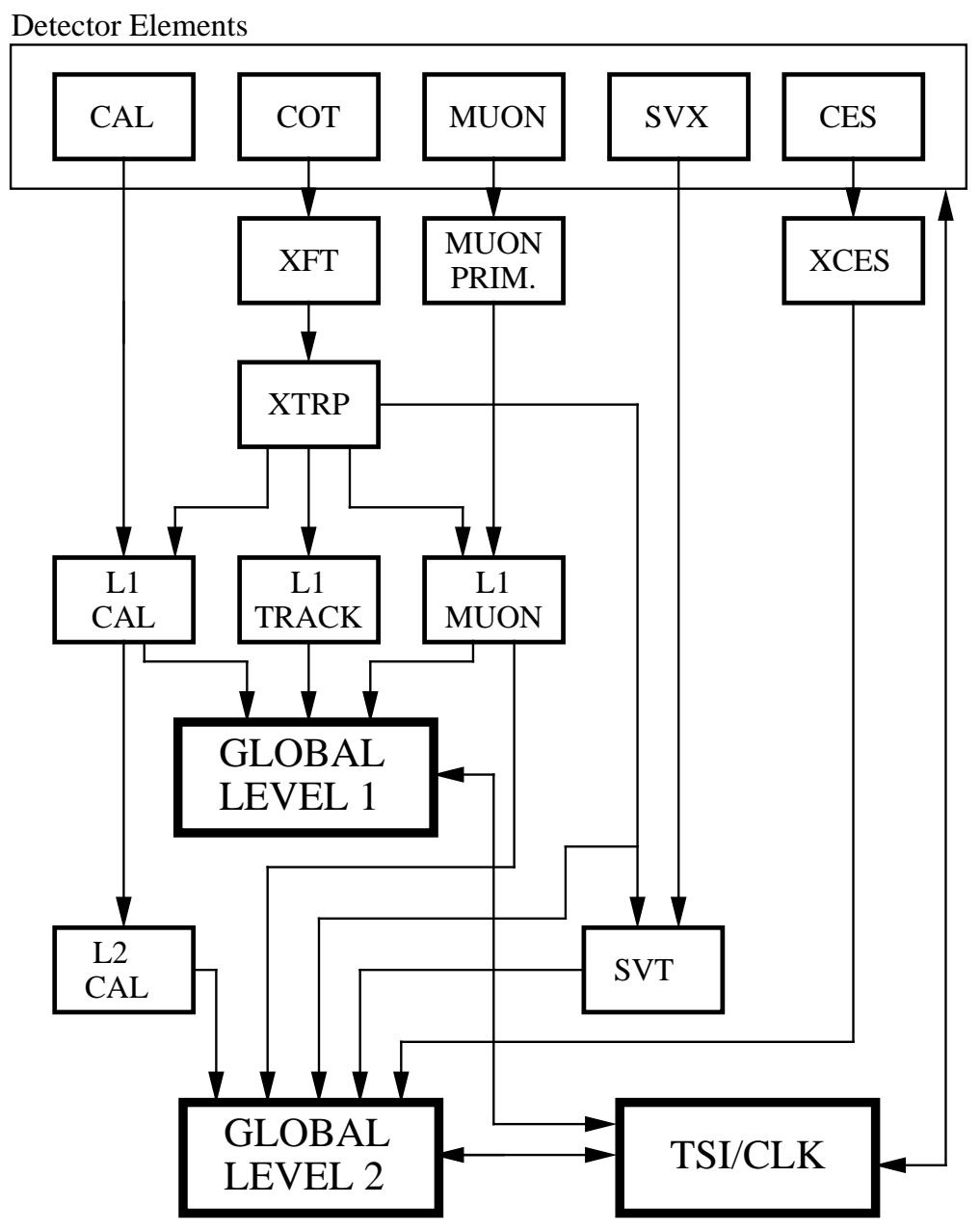

PJW 9/23/96

Figure 3.11: Trigger system flow chart. 
processes, events and cross-sections are generated using an appropriate Monte Carlo event generator. The Monte Carlo events contain information regarding the energy and momenta of incoming and outgoing particles, as described in Section 2.3. This information is then run through CdfSim, which is a GEANT-based simulation of the full CDF detector. This program simulates the behavior of the particles in the detector and the resolutions of the detector components. The output of CdfSim is run through full data reconstruction. At this point, the simulated data "look" like real data. The output can be treated as data, with the exception of applying corrections because the simulation resolution does not perfectly reflect data. These corrections are described further in Chapter 5 and Chapter 6 . 


\section{Chapter 4}

\section{Dataset and Event Selection}

The data used in this analysis are triggered using central electron paths which require the events have at least one high $E_{T}$ central electron candidate. Thus, although we search in both the central and plug detector regions for electron and photon candidates, all of the events in the analysis sample must have at least one central electron. The analysis was split into two searches: a central search and plug search. The central search requires two electrons and a photon be detected in the central region. The plug search requires at least one central electron and one plug object (either the electron or photon); the remaining electron or photon can be central or plug. The results from the central and plug regions are combined. Electrons and photons are identified in the data via "identification" cuts based upon the behavior of electrons and photons in the detector. The electron and photon selection identification requirements in each detector region are discussed in this chapter.

In addition to central electron and photon requirements, we impose another constraint. The dielectron mass $\left(M_{e e}\right)$ must not be between $81 \mathrm{GeV}$ and $101 \mathrm{GeV}$,

the mass window of the $Z$ boson resonance. This requirement helps reject $Z+\gamma$ and $Z+j e t$ events which are the major sources of background in this analysis. The effectiveness of the $M_{e e}$ requirement is discussed further in Section 9.10. 
All data are reprocessed using CDF software version 4.11.1.

\subsection{Triggers}

The primary dataset used for analysis is collected using the ELECTRON_CENTRAL_18 and ELECTRON70_L2_JET paths [14]. The ELECTRON_CENTRAL_18 triggers on electrons with lower $E_{T}$ requirements than the ELECTRON70_L2_JET path, which has a high $E_{T}$ cut with fewer electron selection cuts applied. This allows a path for higher $E_{T}$ electrons which may otherwise have failed the ELECTRON_CENTRAL_18 trigger selection criteria.

Additionally, QCD enriched datasets are used for background predictions. These datasets are triggered on the inclusive JET_20, JET_50, JET_70, and JET_100 paths.

\subsubsection{ELECTRON_CENTRAL_18 Trigger Path}

The ELECTRON_CENTRAL_18 path triggers at level-1 on L1_CEM8_PT8, at level2 on L2_CEM16_PT8, and at level-3 on L3_ELECTRON_CENTRAL_18.

- Level 1: The L1_CEM8_PT8 trigger requires the presence of a central electromagnetic tower with $E_{T}>8 \mathrm{GeV}$ associated with a track from the eXtremely Fast Tracker (XFT track) with $P_{T}>8.34 \mathrm{GeV}$. The XFT track must have at least 10 (or 11) hits in at least 3 (or 4) layers.

- Level 2: The L2_CEM16_PT8 level-2 trigger requires a central electromagnetic cluster with $E_{T}>16 \mathrm{GeV}$ from the "high $E_{T}$ " clustering with an XFT track pointing at the seed tower. Additionally, the ratio of hadronic energy to 
electromagnetic energy $\left(\frac{E_{H a d}}{E_{E M}}\right)^{1}$ in the cluster must not exceed 0.125 .

- Level 3: The L3_ELECTRON_CENTRAL_18 requires an offline calorimeter cluster with $E_{T}>18 \mathrm{GeV}$ associated with a COT track with transverse momentum greater than $9 \mathrm{GeV}$. As in level-2, the cluster must have $\frac{E_{H a d}}{E_{E M}}<$ 0.125. Data taken after January 2003 have the following additional requirements $^{2}: L_{s h r}<0.4,|\Delta Z|<8 \mathrm{~cm}$. Additionally, the transverse component of the energy is computed using the track direction rather than $Z=0$. Also, 3 hadronic towers are used in the $\frac{E_{H a d}}{E_{E M}}$ calculation rather than 2.

\subsubsection{ELECTRON70_L2_JET Trigger Path}

The ELECTRON70_L2_JET path triggers on L1_JET10 at level-1, on L2_JET90 at level-2, and on L3_ELECTRON70_CENTRAL at level-3.

- Level 1: The L1_JET10 level-1 trigger requires a single tower with electromagnetic plus hadronic energy greater than $10 \mathrm{GeV}$.

- Level 2: The L2_JET90 requires a jet with electromagnetic plus hadronic transverse energy greater than $90 \mathrm{GeV}$.

- Level 3: A fully reconstructed offline calorimeter cluster with $E_{T}>70 \mathrm{GeV}$ with a matched COT track with $p_{T}>15 \mathrm{GeV}$ and $\frac{E_{H a d}}{E_{E M}}<0.2+0.001 * E_{E M}$ is required to pass L3_ELECTRON70_CENTRAL trigger. For data taken after January 2003, the $\frac{E_{H a d}}{E_{E M}}$ measurement included 3 hadronic towers rather than 2.

\footnotetext{
$1 \frac{E_{H a d}}{E_{E M}}$ is a quantity that is used to identify electrons. It is defined in Section 4.4

${ }^{2} L_{s h r}$ and $|\Delta Z|$ are quantities that are used to identify electrons. They are defined in Section 4.4
} 


\subsubsection{JET_20, JET_50, JET_70, and JET_100 Trigger Paths}

The JET_20, JET_50, JET_70, and JET_100 trigger paths are used as a source of enriched QCD data for measuring backgrounds. Because they are similar trigger paths, we discuss them concurrently. The trigger names at each level for each trigger path are succinctly summarized in Table 4.1.

\begin{tabular}{|l|c|c|c|}
\hline Trigger Path & $\begin{array}{c}\text { Level 1 } \\
\text { Trigger Name }\end{array}$ & $\begin{array}{c}\text { Level 2 } \\
\text { Trigger Name }\end{array}$ & $\begin{array}{c}\text { Level 3 } \\
\text { Trigger Name }\end{array}$ \\
\hline \hline JET_20 & L1_JET5_PS20 & L2_JET15_PS12 & L3_JET_20 \\
\hline JET_50 & L1_JET5_PS20 & L2_JET40 & L3_JET_50 \\
\hline JET_70 & L1_JET10 & L2_JET60_PS8 & L3_JET_70 \\
\hline JET_100 & L1_JET10 & L2_JET90 & L3_JET_100 \\
\hline
\end{tabular}

Table 4.1: Names of triggers for each level of the JET_20, JET_50, JET_70, and JET_100 trigger paths.

- Level 1: The JET_20 and JET_50 trigger path at level-1 requires that the highest transverse energy tower have total $E_{T}(\mathrm{EM}+\mathrm{HAD})$ greater than 5 $\mathrm{GeV}$. The JET_20 trigger has a prescale ${ }^{3}$ set to 20 , while the JET_50 prescale is set to 12 . The JET_70 and JET_100 level-1 trigger requires a single tower with total $(\mathrm{EM}+\mathrm{HAD})$ transverse energy greater than $10 \mathrm{GeV}$ with no prescale imposed.

- Level 2: At level-2, a jet clustering pass is done. The total jet $E_{T}(\mathrm{EM}+\mathrm{HAD})$ must be greater than $15 \mathrm{GeV}, 40 \mathrm{GeV}, 60 \mathrm{GeV}$, and $90 \mathrm{GeV}$ for JET_20, JET_50, JET_70, and JET_100, respectively. The JET_20 and JET_70 trigger paths have prescales at level-2 set to 12 and 8 , respectively.

\footnotetext{
${ }^{3} \mathrm{~A}$ prescale of $n$, where $n$ is an integer, means that 1 out of every $n$ events passing the criteria is accepted, the rest are rejected. This reduces the total number of events recorded for processes with very high cross-sections.
} 
- Level 3: Fully reconstructed jet clustering is done with a cone of $R=0.7$ in $\eta-$ $\phi$ space using $Z_{v t x}=0$, for simplicity. The minimum $E_{T}$ of the reconstructed jet is $20 \mathrm{GeV}, 50 \mathrm{GeV}, 70 \mathrm{GeV}$, and $100 \mathrm{GeV}$ for the JET_20,JET_50, JET_70, and JET_100 trigger paths, respectively.

\subsection{Central and Plug Electromagnetic Clustering Algorithms}

The offline data reconstruction code reconstructs photons and electrons from the data in two steps [16]. The first step involves identifying electromagnetic "clusters". The second step creates a "CdfEmObject", which is an EM cluster with associated detector objects. For example, in the central region, after EM clusters are found, the code attempts to find tracks, central electromagnetic shower-max clusters, and central pre-radiator clusters which spatially match the EM cluster.

The algorithm for the reconstruction of central EM clusters follows. Central EM clusters are formed by searching the calorimeter towers for "seed" towers, which are defined as towers with electromagnetic transverse energy greater than $3 \mathrm{GeV}$, where the transverse energy is initially calculated using $Z=0.0$. All seed towers are ordered by descending EM transverse energy. Then, the code attempts to attach "shoulder" towers to the seed towers. A shoulder tower is defined as a tower adjacent to the seed tower in $\eta$ with non-zero electromagnetic or hadronic energy. A central electromagnetic cluster will not cross the tower boundary in $\phi$, so all towers will be in the same wedge in $\phi$. Towers which are designated as shoulder towers in a cluster are removed from the list of candidate seed towers, and both the seed and

shoulder towers are added to the "used" towers list. This eliminates the possibility 
that a tower could be used in two clusters.

The preceding algorithm results in the following central electromagnetic cluster properties. A central electromagnetic cluster will consist of, at most, three towers. All towers in a cluster must must be located within the same half of the detector (east or west) and within the same detector region (central or plug). Thus, if the seed tower is located in either tower 0 or tower 9 , the inner and outermost towers, then the cluster can have at most two towers.

Plug electromagnetic clustering is performed using a method called BF_PEM Clustering, or "brute force" clustering. This method is similar to central clustering. First, seed towers with transverse electromagnetic energy greater than $3 \mathrm{GeV}$ are identified. These seed towers are clustered to a "daughter" tower, which is defined as the maximum $E_{T}$ tower bordering the seed tower with EM $E_{T}$ greater than 100 $\mathrm{MeV}$. If found, the daughter tower is removed from the seed tower list and added to the "used" tower list, so as not to be included in another cluster. Following the appointment of a seed and daughter tower, the towers bordering the seed and daughter towers are searched for a pair of tertiary towers with transverse energy greater than $100 \mathrm{MeV}$. The pair with maximum transverse energy is added to the seed and daughter towers. In general, PEM clusters will have a $2 \times 2$ tower structure, however there are exceptions where it is possible to have a 3 tower PEM cluster.

\subsection{CDF Electron and Photon Objects}

Electron and Photon objects, collectively called CdfEMObjects, are created following EM clustering. This is when the offline reconstruction code attempts to associate tracks, shower max clusters, pre-radiator clusters, and other objects used in EM object identification to the EM cluster. Requirements pertinent to this analysis are 
discussed.

Tracks are associated with central EM clusters by extrapolating each track to the radius of the CES. To be associated with the EM cluster, the track must lie within $25 \mathrm{~cm}$ of the tower center in $r-\phi$ space and within $38 \mathrm{~cm}$ of the seed tower in z. The best matched track, or "maxPtTrack" is a track with at least one COT axial hit ${ }^{4}$ that is extrapolated to within the seed tower or within $5 \mathrm{~cm}$ of the seed tower boundary in $Z$. If more than one track meets these requirements, the track with maximum transverse momentum is chosen as the best matched track.

CES clusters are associated with an EM cluster if they lie within the same wedge as the cluster, or if they are seeded by one of the associated tracks. There are two CES cluster "views": strip $(Z)$ and wire $(r-\phi)$. For electrons, the best matched strip and wire CES clusters are required to be seeded by the maxPtTrack. The CES strip(wire) cluster best matched to a photon is the maximum energy strip(wire) cluster within $5 \mathrm{~cm}$ of the seed tower boundary.

CPR clusters are used in this analysis for photon fake rate measurements, as described in Section 8.3. CPR clusters that lie within the same wedge as the EM cluster are associated with it. Reference [16] has further information on EM clustering and the making of CdfEmObjects.

\subsection{Central Electron and Photon Identification}

Electrons in the central region of the detector are identified by the presence of an electromagnetic cluster in the calorimeter accompanied by a track in the central outer tracker. Photons create electromagnetic clusters, but do not have associated

\footnotetext{
${ }^{4}$ One COT axial hit is required to avoid matching a silicon-standalone track to an EM cluster. These tracks are often poorly measured.
} 
tracks. Because photons and electrons behave similarly in the calorimeters, they are identified by several of the same variables. The selection criteria for electrons and photons are shown in Tables 4.2 and 4.3, respectively. The following central identification variables are common to both electrons and photons. The kinematic and geometric requirements used to ensure the detection of well measured high $E_{T}$ electrons and photons are discussed first.

\section{- $E_{T}>25 \mathrm{GeV}$}

The energy is defined as the two-tower total EM energy associated with the EM cluster, where the two towers are the seed tower and the nearest tower to the track direction in $\eta$. The transverse energy is defined as the amount of energy in the transverse direction. For electrons, this is determined using the angle $\theta$ that the COT-only beam-constrained maximum- $p_{T}$ track makes with the beam axis. For photons, the amount of photon energy in the transverse direction is determined from the $Z_{0}$ of the track corresponding to the maximum $E_{T}$ central electron in the event. Corrections made to the EM energy include the CEMCORR correction using the track parameters to determine the local coordinates. This correction takes into account tower mapping corrections, tower-to-tower relative gain variations, and the global energy scale of the CEM [15].

- Detector $\eta_{d}$ is central, $\eta_{d}<1$. The EM cluster must lie within the CEM detector region as determined by the electromagnetic cluster seed tower.

\section{- Fiducial}

The fiducial region of the detector is defined as those regions which are well instrumented. We require EM clusters to be "fiducial" to maximize the energy 
resolution and other quantities. The fiduciality is defined using the CES shower local coordinates. For electrons and photons, we require [15]:

$-\left|X_{C E S}\right|<21 \mathrm{~cm}$. The local X position of the shower profile is measured using the CES wires. We require the shower be located within $21 \mathrm{~cm}$ of the tower center in the $r-\phi$ view. As discussed in Chapter 3, each calorimeter wedge spans $15^{\circ}$ in $\phi$. The boundaries of these wedges consist of poorly instrumented regions which are rejected by this cut.

- $9 \mathrm{~cm}<\left|Z_{C E S}\right|<230 \mathrm{~cm}$. The local $Z$ position of the shower profile is measured with the CES strips. This cut excludes the gap between the east and west barrels of the central calorimeter. $\left|Z_{C E S}\right|<230 \mathrm{~cm}$ excludes the outer portion of the outermost central tower, tower 9 , which is prone to energy leakage.

Additionally, electrons are required to pass the following geometric cuts:

- Tower 9 EM clusters are excluded due to energy leakage into the hadronic calorimeter.

- Allow Tower 7 of wedge 05E (chimney) only if local $Z<193.0 \mathrm{~cm}$. This corresponds to the region of the detector near the "chimney". The chimney houses the cryogenic connections to the solenoid and thus is not well instrumented. This exclusion region corresponds to $0.77<\eta<1.0$ for $75^{\circ}<\phi<90^{\circ}$.

These fiducial requirements leave $\sim 75 \%$ of the CEM available for measurements. 
Electrons and photons shower similarly in the calorimeter detectors. Hence, the calorimeter identification quantities $\frac{E_{H a d}}{E_{E M}}$ and calorimeter isolation are variables used for both electron and photon selection.

- $\frac{E_{H a d}}{E_{E M}}$

Electron and photons deposit the majority of their energy in the electromagnetic calorimeter with minimal energy deposited in the hadronic calorimeter, while hadronic jets deposit energy primarily in the hadronic calorimeters. Thus, a cut on the ratio of energy deposition in the hadronic region to the energy deposition in the electromagnetic region can be used to reject hadronic jets. We use a sliding cut with the energy of the EM cluster because the amount of leakage into the hadronic calorimeter increases with energy. This allows us to maintain the identification efficiency of true electrons and photons. Photons pass the $E_{H a d} / E_{E M}$ requirement $E_{H a d} / E_{E M}<0.125$ or if it passes the sliding cut. Electrons must pass the sliding cut. The sliding cut is given by:

$$
\frac{E_{H a d}}{E_{E M}}<(0.055+0.0045 \cdot E)
$$

- Calorimeter Isolation $(\Delta R<0.4)$

The presence of additional energy in the calorimeter near the electromagnetic cluster indicates that the cluster was likely formed by a jet rather than an electron. Thus we require that the electromagnetic cluster be well separated from other energy in the calorimeter. Calorimeter isolation is defined as the sum of the total (electromagnetic + hadronic) transverse energy in a cone of radius 0.4 in $\eta-\phi$ space, $\Delta R=\sqrt{\Delta \eta^{2}+\Delta \phi^{2}}$, surrounding the EM cluster centroid, excluding the energy of the cluster. The isolation is corrected for 
energy leakage from the electron into neighboring towers in $\eta$. A cut on the ratio of the isolation energy to the EM cluster energy is employed:

$$
I s o(\Delta R<0.4)=\frac{E_{T}^{0.4}-E_{T}}{E_{T}}<0.1
$$

\subsection{Central Electron Identification}

The variables unique to central electron identification are discussed now. These include track $p_{T}, \frac{E}{p},|\Delta X|,|\Delta Z|$, and track $Z_{\circ}$. Electrons with transverse energy less than $100 \mathrm{GeV}$ are selected with a slightly tighter set of cuts than those with greater transverse energy. This maximizes the identification efficiency in the high $E_{T}$ search region where background contamination is low.

- $p_{T}$

The electron's track is defined as the track with maximum $P_{T}$ that is associated with the EM cluster. The track must have transverse momentum greater than $10 \mathrm{GeV}$. Track quantities are determined using only the COT information (no silicon information). Additionally, the track is constrained to the $x-y$ location of the beam. The transverse momentum is calculated using the curvature of the track (Equation 3.3).

- $\frac{E}{p}$

The Energy-Momentum ratio of a relativistic electron should be close to 1.0 since it has negligible mass. Deviations from this value occur. Bremsstrahlung radiation will decrease the measured track momentum. However, the radiated photon will typically be contained within the electron electromagnetic cluster 
and thus be included in the energy measurement. As a result, the energymomentum ratio will be greater than 1 and result in a tail in the distribution. Loss of energy due to cracks in the calorimeter will decrease this ratio. We require $\frac{E}{p}<4$ for $E_{T}<100 \mathrm{GeV}$ and no cut for $E_{T}>100 \mathrm{GeV}$.

\section{- $L_{s h r}$}

$L_{s h r}$ (pronounced $L$-share) is another variable that helps differentiate EM clusters formed by hadronic jets from those resulting from electrons. The lateral shower profile is a measure of the lateral sharing of energy across adjacent towers. It is, in a sense, an isolation cut. It is defined as:

$$
L_{s h r}=0.14 \cdot \sum_{i} \frac{E_{i}^{a d j}-E_{i}^{e x p}}{\sqrt{(0.14)^{2} \cdot E+\left(\Delta E_{i}^{e x p}\right)^{2}}}
$$

where $E_{i}^{a d j}$ is the amount of energy measured in a tower adjacent to and within the same wedge as the seed tower, $E_{i}^{e x p}$ is the predicted energy deposited in that tower, $\mathrm{E}$ is the total EM energy of the cluster, and $\Delta E_{i}^{e x p}$ is the uncertainty on $E_{i}^{e x p}$. The CES is seeded using the track associated with the EM cluster and the sum is over 3 towers.

\section{- $|\Delta X|$}

The track associated with the electron is extrapolated to the CES. $\Delta X$ is defined as the distance between the extrapolated track position and the local CES shower position in the $r-\phi$ view. We require they be at most $3 \mathrm{~cm}$ apart.

- $|\Delta Z|$

The track associated with the electron is extrapolated to the CES. The $Z$ 
position of the track is required to lie within $5 \mathrm{~cm}$ of the CES shower $Z$ coordinate. CES alignment corrections are taken into account for both $|\Delta X|$ and $|\Delta Z|$ quantities.

- Track $Z_{0}$

Track $Z_{0}$ is the COT-only beam constrained $Z$ coordinate of the track associated with the electron, at the point of closest approach to the beam.

\begin{tabular}{|l|c|c|}
\hline Variable & \multicolumn{2}{|c|}{ Selection Criteria } \\
\hline \hline & $E_{T}<100 \mathrm{GeV}$ & $E_{T}>100 \mathrm{GeV}$ \\
\hline$E_{T}$ & $>25 \mathrm{GeV}$ & $>100 \mathrm{GeV}$ \\
\hline Track $p_{T}$ & $>10 \mathrm{GeV}$ & $>25 \mathrm{GeV}$ \\
\hline$\frac{\text { Cal Iso( }\left(\Delta_{R}<0.4\right)}{E_{T}}$ & $<0.1$ & $<0.1$ \\
\hline$\frac{E_{H a d}}{E_{E M}}$ & $<0.055+0.00045 \cdot E$ & $<0.055+0.00045 \cdot E$ \\
\hline$\frac{E}{p}$ & $<4.0$ & - \\
\hline$L_{s h r}$ & $<0.2$ & - \\
\hline$|\Delta X|$ & $<3 \mathrm{~cm}$ & $<3 \mathrm{~cm}$ \\
\hline$|\Delta Z|$ & $<5 \mathrm{~cm}$ & $<5 \mathrm{~cm}$ \\
\hline Track $\left|Z_{0}\right|$ & $<60 \mathrm{~cm}$ & $<60 \mathrm{~cm}$ \\
\hline
\end{tabular}

Table 4.2: Central electron selection criteria.

\subsection{Central Photon Identification}

Photons in the central region of the detector are identified by the presence of an electromagnetic cluster in the calorimeter and the absence of a track in the COT. The $E_{H a d} / E_{E M}$ and calorimeter isolation variables were described in Section 4.5. The selection criteria for photons are summarized in Table 4.3.

- The transverse energy for photons is defined by the the two-tower electromagnetic energy and the angle made from the $\left|Z_{0}\right|$ of the track associated with 
the highest $E_{T}$ central electron in the event to the location of the photon CES cluster.

- The fiducial requirements were described in Section 4.5. The local CES X shower position must be less than $21 \mathrm{~cm}$ from the center of the tower. The $\left|Z_{\text {local }}\right|$ shower position must be between $9 \mathrm{~cm}$ and $230 \mathrm{~cm}$.

- N3d Tracks

Photons require the absence of a COT track associated with an electromagnetic cluster. The N3d cut requires there be no 3D track in the same direction as the EM cluster, or, if a track exists, its transverse momentum be less than $1 \mathrm{GeV}+0.005 \cdot E_{T}$.

- Track Isolation

A cluster which passes the N3d track requirement but still has tracks in its vicinity likely means that it was formed by a jet. We define tracking isolation as the scalar sum of the transverse momenta of all tracks in a cone of 0.4 in $\eta-\phi$ space about the cluster centroid. We require track isolation be less than $5 \mathrm{GeV}+0.005 E_{T}$

- $\chi^{2}$

The $\chi^{2}$ measurement provides a comparison of the electron and photon transverse shower profile in the CES to what is expected from test beam studies. There is a $\chi^{2}$ measurement in the strip $(Z)$ and wire $(r-\phi)$ view. The cut is on the average of the two measurements: $\left(\chi_{\text {strip }}^{2}+\chi_{\text {wire }}^{2}\right) / 2<20$.

- 2nd CES Cluster Energy 
The second CES Cluster energy is the energy of the second highest strip or wire CES cluster matched to the EM cluster. This quantity is multiplied by $\sin \theta$ to account for the amount of material traversed by the photon. This cut removes photons due to neutral meson decay such as $\pi^{0} \rightarrow \gamma \gamma$ events.

\begin{tabular}{|l|c|}
\hline Variable & Selection Criteria \\
\hline \hline$E_{T}$ & $>25 \mathrm{GeV}$ \\
\hline Fiducial & Ces $|X|<21 \mathrm{~cm}$ \\
& $9<\mathrm{Ces}|Z|<230 \mathrm{~cm}$ \\
\hline N3d Tracks & $\begin{array}{c}<=1, \text { where the track has } \\
P_{T}<1 \mathrm{GeV}+0.005 \cdot E_{T}\end{array}$ \\
\hline Calorimeter Iso $E_{T}(\Delta R<0.4)$ & $<0.1 \cdot E_{T}$ \\
\hline Track Iso $p_{T}(\Delta R<0.4)$ & $<5 . \mathrm{GeV}+0.005 \cdot E_{T}$ \\
\hline$\frac{E_{H a d}}{E_{E M}}$ & $<0.055+0.00045 \cdot E$ \\
& or $<0.125$ \\
\hline Average $\chi^{2}$ & $<20$ \\
\hline 2nd CES Cluster Energy $\times \sin \theta$ & $<2.4 \mathrm{GeV}+0.01 \cdot E_{T}$ \\
\hline
\end{tabular}

Table 4.3: Central photon selection criteria.

\subsection{Plug Electron and Photon Identification}

The central outer tracker does not have significant coverage in the plug $(1.1<|\eta|<$ 2.8) region. Therefore, no track requirements are placed on plug region EM clusters for photon or electron identification. The calorimeter response to electrons is similar to photons. Thus, electrons and photons are treated as the same objects in the plug detector region, a PEM of plug EM object. PEM objects must pass the selection criteria in Table 4.4 .

This analysis requires at least one central electron. The track vertex associated with the electron with maximum transverse energy is used to determine the 
transverse plug quantities: $E_{T}$ and calorimeter isolation.

The calorimeter quantities $\frac{E_{H a d}}{E_{E M}}$ and calorimeter isolation have the same definition for plug electromagnetic objects as for central objects. The PEM $\chi^{2}$ provides a measurement of the pattern of electromagnetic energy distributed in the $3 \times 3$ calorimeter towers about the seed tower compared to the expected distribution as predicted by test beam data.

\begin{tabular}{|l|c|}
\hline Variable & Selection Cut \\
\hline \hline$|\eta|$ & $1.2-2.8$ \\
\hline$E_{T}$ & $>25 \mathrm{GeV}$ \\
\hline$\frac{\text { CalIso( }(\Delta R<0.4)}{E_{T}}$ & $<0.1$ \\
\hline$\frac{E_{H a d}}{E_{E M}}$ & $<0.055+0.00045 \cdot E$ \\
\hline $\mathrm{PEM} \chi^{2}$ & $<10$ \\
\hline
\end{tabular}

Table 4.4: Plug EM object selection criteria.

\section{8 e $e \gamma$ and eer Combinations}

We primarily study two invariant mass spectra: the two body $\left(M_{e \gamma}\right)$ and the three body $\left(M_{e e \gamma}\right)$. Events with 0 or 1 plug EM objects will have two entries in the $M_{e \gamma}$ distribution, one for each possible $e \gamma$ combination. Events with a central electron and 2 plug EM objects have three entries in the $M_{e \gamma}$ distribution due to the ambiguity in the plug electron - photon identification. Every event will have only one entry in the $M_{e e \gamma}$ plots. 


\section{Chapter 5}

\section{Energy Corrections}

The data used in this analysis are reprocessed using CDF software version 4.11.1. Several energy corrections are applied to EM objects during the reprocessing, such as tower-to-tower gain corrections and face corrections.

The tower-to-tower corrections refer to corrections necessary due to the unique response of each tower. The gain differences between towers is determined and corrected for, resulting in about 5\% improvement in energy resolution [17].

Additionally, the energy response produced by an electromagnetic object is affected by its location within a tower. Thus, the location of the CES cluster associated with the electromagnetic cluster is used to correct for the position of the cluster within the calorimeter tower. These "face" corrections are applied during reconstruction of electromagnetic clusters.

Several other energy corrections are applied, including for the attenuation of light as it passes through the scintillator. This effect changes with time and must be corrected as a function of run number.

These corrections are not sufficient to accurately measure the energy of electromagnetic objects; we must also scale EM object energies at ntuple-level.

This chapter covers the ntuple-level energy scales applied to EM objects in the data and the energy smearing corrections applied in the simulation. We also 
summarize the systematics on the energy scales and energy smearing corrections. The same corrections are applied to electrons and photons since they exhibit similar behavior in the calorimeters.

\subsection{Energy Scales}

\subsubsection{Central Electrons and Photons}

Figure 5.1(a) shows the dielectron mass distribution for central-central $Z \rightarrow e e$ candidates compared to simulated $Z \rightarrow e e$ events. The data $Z$ boson mass peaks at $(90.99 \pm 0.08) \mathrm{GeV}$ with width $(3.79 \pm 0.09) \mathrm{GeV}$. Thus, we need to scale the EM

cluster energies in the data up such that the peak is at $91.19 \mathrm{GeV}$, the known value of the $Z$ boson mass.

Figure 5.1(b) shows the average dielectron mass as a function of run number. It is a fairly flat distribution, indicating that a constant scale factor is nearly sufficient for correcting the energy of EM clusters. However, there are slight variations and we scale the data differently for three time periods:

- Pre-January 2003 (Runs 141544-156487)

- January-May 2003 (Runs 159603-163527)

- May-September 2003 (Runs 163527-168889)

The choice of time periods is motivated by the December 2002 to January 2003 shut-down when various components of the CDF detector were worked on. The reason for the May-September 2003 time period is that the post-May calibrations may not be completely up-to-date in the database at the time this data were ntupled. 


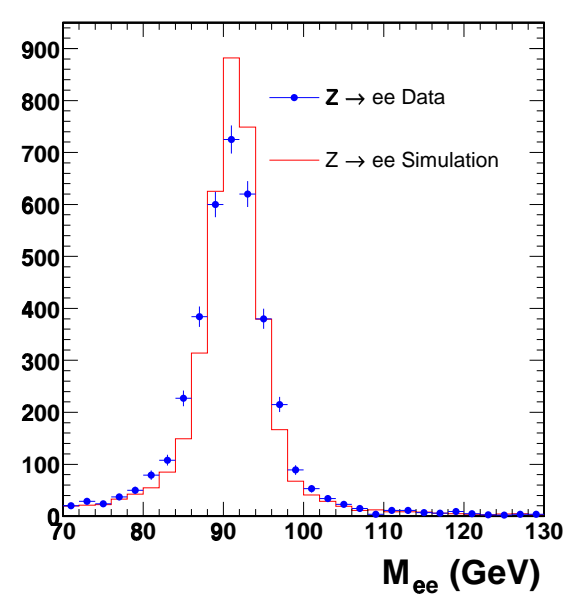

(a)

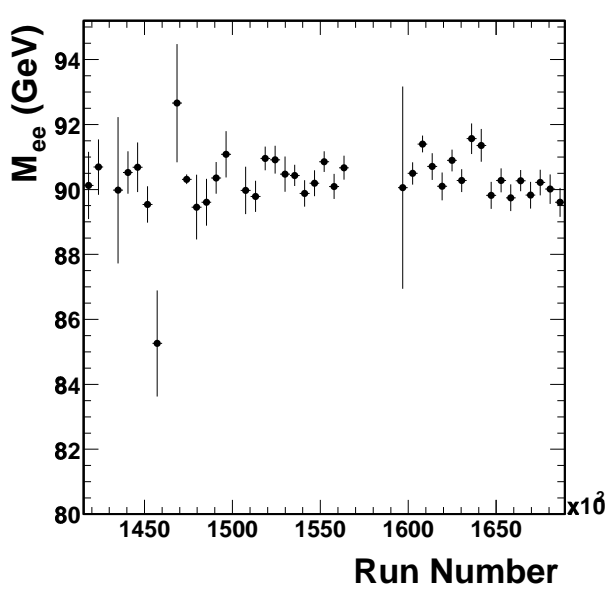

(b)

Figure 5.1: Figure (a) shows the dielectron mass distribution for $Z \rightarrow e e$ centralcentral candidate events in simulation and data before corrections. Figure (b) shows the average dielectron mass as a function of run number before corrections.

We scale the data such that the $Z$ mass peaks at $91.19 \mathrm{GeV}$. To obtain this, the energies are scaled as shown in Table 5.1. The $Z$ mass peak after applying scale factors (integrated over all runs) is $(91.18 \pm 0.08) \mathrm{GeV}$ and width is $(3.78 \pm 0.09)$ $\mathrm{GeV}$. The effect of the energy scale corrections is shown in Figure 5.3.

Table 5.1: Energy scale factors applied to central EM objects

\begin{tabular}{|l|c|c|c|}
\hline Run Period & $\begin{array}{c}M_{e e} \text { Before } \\
\text { Scale Factor }\end{array}$ & $\begin{array}{c}\text { Scale Factor } \\
\text { Applied }\end{array}$ & $\begin{array}{c}M_{e e} \text { After } \\
\text { Scale Factor }\end{array}$ \\
\hline \hline $141544-156487$ & $90.98 \pm 0.10 \mathrm{GeV}$ & 1.003 & $91.20 \pm 0.10 \mathrm{GeV}$ \\
\hline $159603-163527$ & $91.19 \pm 0.15 \mathrm{GeV}$ & 1.000 & $91.19 \pm 0.15 \mathrm{GeV}$ \\
\hline $163527-168889$ & $90.70 \pm 0.13 \mathrm{GeV}$ & 1.005 & $91.16 \pm 0.14 \mathrm{GeV}$ \\
\hline
\end{tabular}




\subsubsection{Plug EM Objects}

The dielectron invariant mass for central-plug $Z \rightarrow e e$ candidate events is $(89.75 \pm$ 0.09) $\mathrm{GeV}$ when the plug object is located in the west barrel. The same quantity for east plug objects is $(89.48 \pm 0.11) \mathrm{GeV}$. Both quantities already have the aforementioned central energy scale applied to the central object. We study the data to determine the appropriate energy corrections for plug EM objects, with separate corrections in the east and west calorimeters.

Figures 5.2(a) and 5.2(b) show the dielectron mass distribution of central-plug $Z \rightarrow e e$ candidates in the west and east calorimeters. Figure 5.2(c) and 5.2(d) show the average dielectron mass of central-plug $Z \rightarrow e e$ candidates as a function of run number. Again, we scale the data such that the dielectron mass peaks at the known $Z$ mass of $91.19 \mathrm{GeV}$. The scales used in the three run periods are shown in Tables 5.2 and 5.3.

The effect of the energy scale corrections are demonstrated in Figure 5.4. As expected, the mass peak is located at $91.2 \mathrm{GeV}$. Additionally, the reconstructed $Z$ mass is now run-independent.

Table 5.2: Energy scale factors applied to west plug EM objects

\begin{tabular}{|l|c|c|c|}
\hline Run Period & $\begin{array}{c}M_{e e} \text { Before } \\
\text { Scale Factors }\end{array}$ & West Scale Factor & $\begin{array}{c}M_{e e} \text { After } \\
\text { Scale Factors }\end{array}$ \\
\hline \hline $141544-156487$ & $88.83 \pm 0.12 \mathrm{GeV}$ & 1.052 & $91.18 \pm 0.12 \mathrm{GeV}$ \\
\hline $159603-163527$ & $87.52 \pm 0.16 \mathrm{GeV}$ & 1.082 & $91.16 \pm 0.16 \mathrm{GeV}$ \\
\hline $163527-168889$ & $87.40 \pm 0.14 \mathrm{GeV}$ & 1.089 & $91.21 \pm 0.16 \mathrm{GeV}$ \\
\hline
\end{tabular}




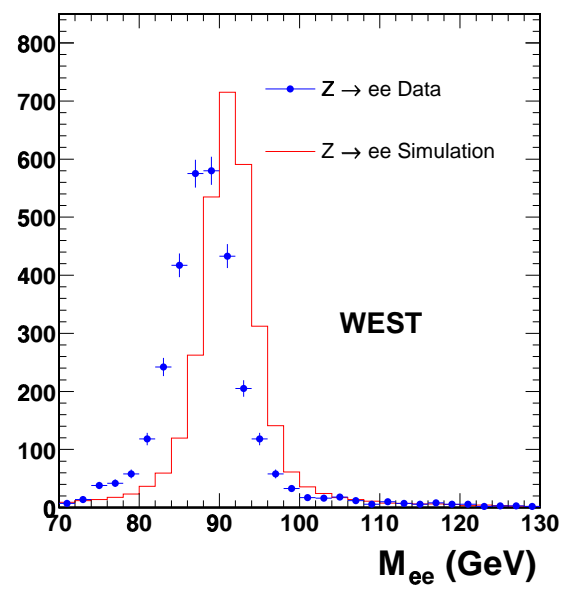

(a)

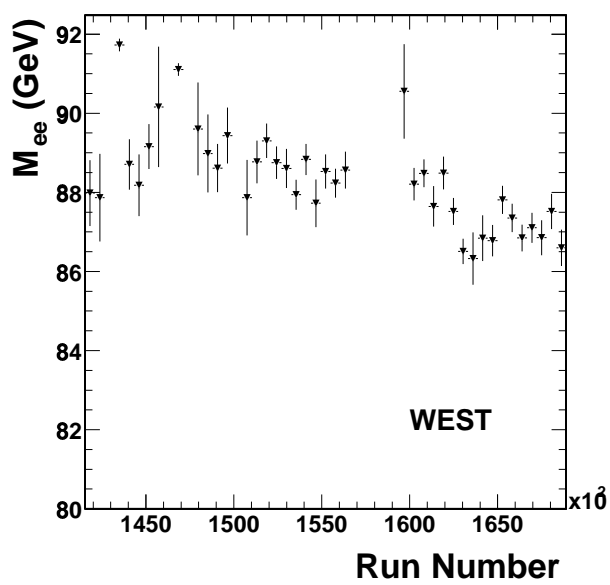

(c)

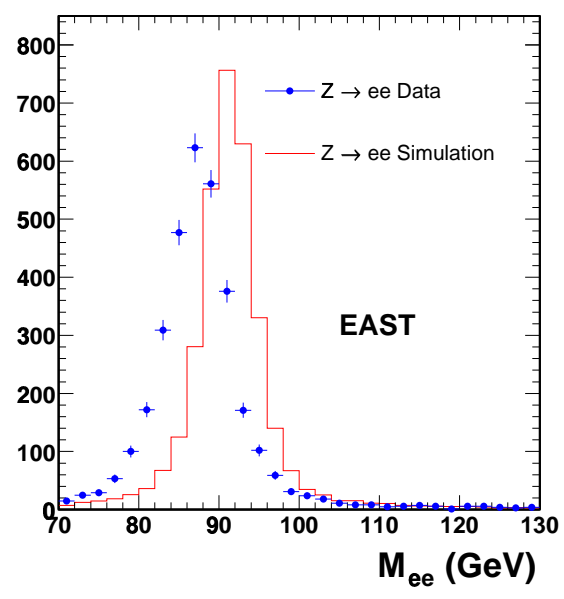

(b)

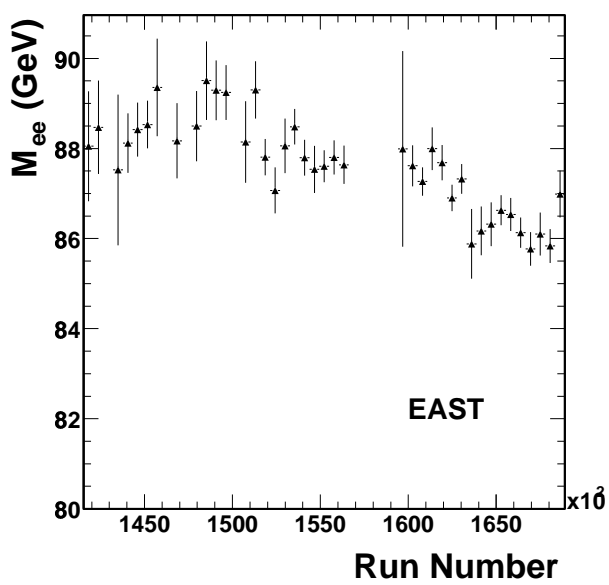

(d)

Figure 5.2: Figure (a) and (b) show the data-simulation comparisons for the $Z$ mass distribution for Central-Plug events before energy corrections, for the west and east barrels, respectively. Figure (c) and (d) show the average $Z$ mass peak as a function of run number, for the west and east barrels, respectively. 
Table 5.3: Energy scale factors applied to east plug EM objects

\begin{tabular}{|l|c|c|c|}
\hline Run Period & $\begin{array}{c}M_{e e} \text { Before } \\
\text { Scale Factors }\end{array}$ & East Scale Factor & $\begin{array}{c}M_{e e} \text { After } \\
\text { Scale Factors }\end{array}$ \\
\hline \hline $141544-156487$ & $88.43 \pm 0.11 \mathrm{GeV}$ & 1.062 & $91.17 \pm 0.12 \mathrm{GeV}$ \\
\hline $159603-163527$ & $87.02 \pm 0.15 \mathrm{GeV}$ & 1.096 & $91.14 \pm 0.16 \mathrm{GeV}$ \\
\hline $163527-168889$ & $86.28 \pm 0.15 \mathrm{GeV}$ & 1.116 & $91.17 \pm 0.17 \mathrm{GeV}$ \\
\hline
\end{tabular}

\subsection{Energy Resolution}

The energy resolution in simulation is typically better than the resolution observed in data. The width of the $Z$ mass distribution is used to better match the simulated energy resolution to the the data. The energy of each EM object is multiplied by $(1+\kappa)$, where $\kappa$ is a Gaussian random number with mean 0 and width equal to the energy smearing correction for the given object. A value for $\kappa$ is generated for each object on an event-by-event basis.

\subsubsection{Central Electrons and Photons}

The width of the $Z$ peak in data integrated over all runs, after applying the energy scale factors, is $(3.78 \pm 0.09) \mathrm{GeV}$. The simulated width of the $Z$ mass distribution is $(3.11 \pm 0.02) \mathrm{GeV}$. To better match the energy resolution observed in the data, a smearing correction factor of 0.0317 , or $3.17 \%^{1}$, is applied to the energies of central electrons and photons. This smearing results in a simulated $Z$ width of $(3.77 \pm 0.03) \mathrm{GeV}$. The $Z$ mass distributions resulting from applying the data energy scale correction and simulation energy smearing corrections are shown in Figure 5.3.

\footnotetext{
${ }^{1}$ The smearing correction factors will now be quoted as percentages (\%).
} 


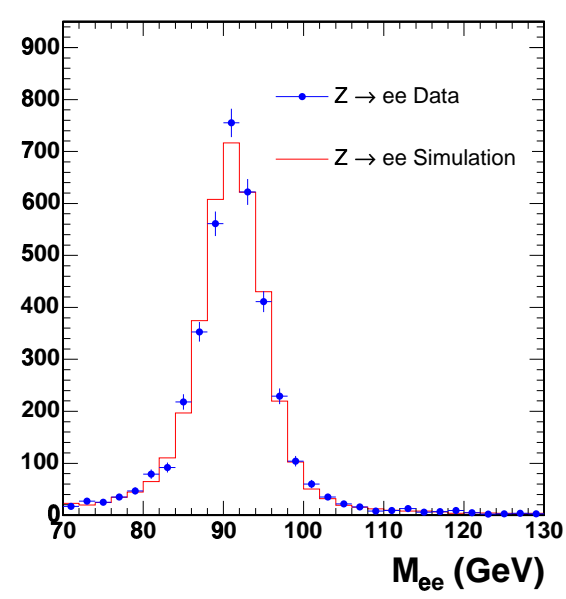

(a)

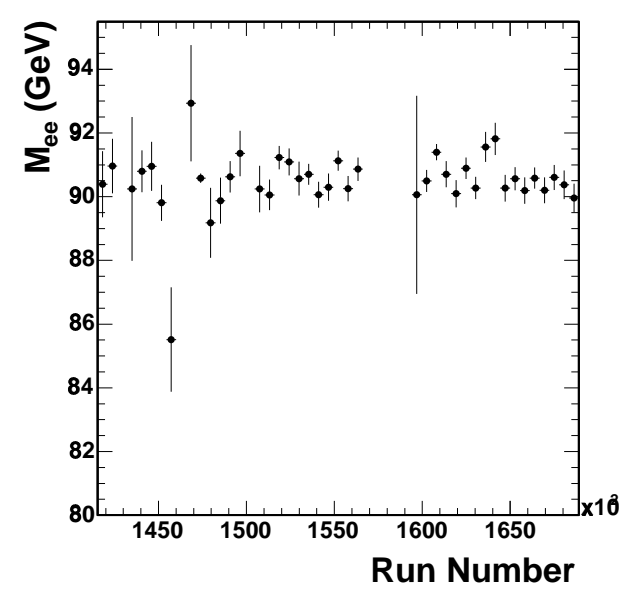

(b)

Figure 5.3: Figure (a) shows the dielectron mass distribution for $Z \rightarrow e e$ centralcentral candidate events in simulation and data after energy scale and smearing corrections. Figure (b) shows the average dielectron mass as a function of run number after energy scale corrections.

\subsubsection{Plug EM Objects}

Figure 5.2 shows that the energy resolution of the simulation does not agree with the data. The width of the $Z$ mass distribution in data is $(3.56 \pm 0.08) \mathrm{GeV}$ for west plug events and $(3.63 \pm 0.09) \mathrm{GeV}$ for east plug events. The widths produced by the simulation are $(3.14 \pm 0.02) \mathrm{GeV}$ in the west and $(3.12 \pm 0.02) \mathrm{GeV}$ in the east. The smearing corrections to the west and east plug calorimeter energies are determined separately and are found to be equal to $1.9 \%$ for both west and east plug objects. This is summarized in Table 5.4.

After the data energy scale factors in Tables 5.2 and 5.3 and the simulation energy smearing corrections in Table 5.4 are applied, the $Z$ mass distributions agree well between data and Monte Carlo. This is demonstrated in Figure 5.4. 


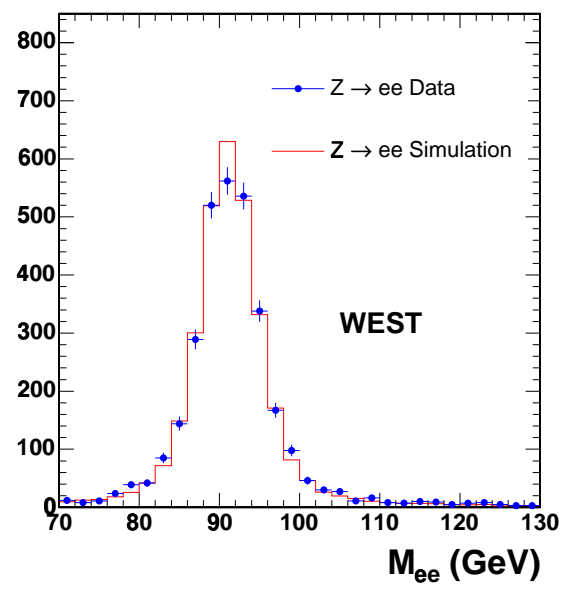

(a)

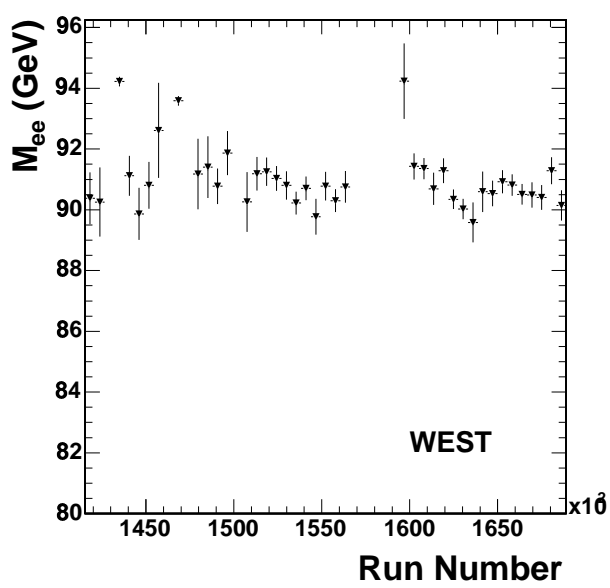

(c)

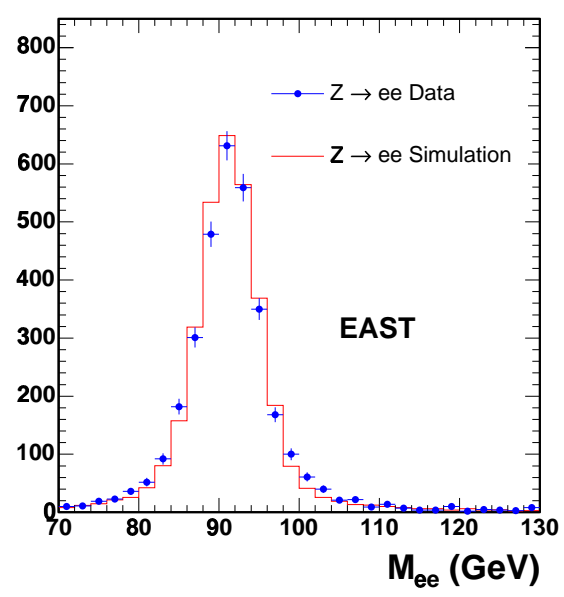

(b)

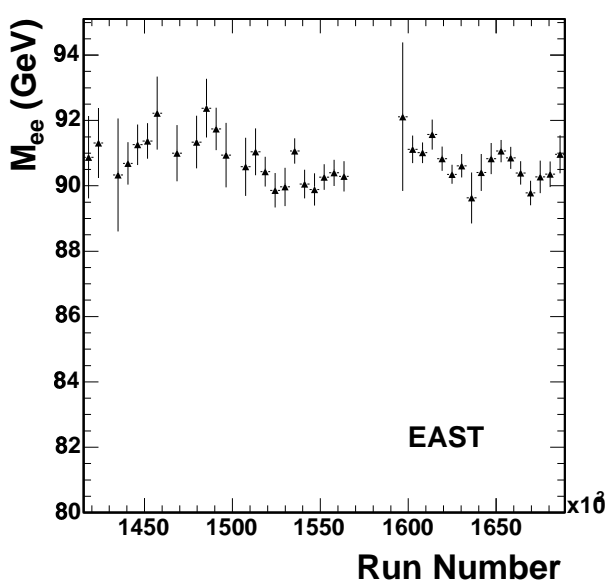

(d)

Figure 5.4: Figure (a) and (b) show the data-simulation comparisons for the $Z$ mass distribution for central-plug events after energy corrections, for the west and east barrels, respectively. Figure (c) and (d) show the average $Z$ mass peak as a function of run number, for the west and east barrels, respectively, after energy corrections. 
Table 5.4: Energy smearing applied to simulated plug EM objects

\begin{tabular}{|l|c|c|c|c|}
\hline Barrel & $\begin{array}{c}Z \text { Width Before } \\
\text { Smearing }\end{array}$ & $\begin{array}{c}\text { Extra } \\
\text { Smearing (\%) }\end{array}$ & $\begin{array}{c}Z \text { Width After } \\
\text { Smearing }\end{array}$ & $\begin{array}{c}Z \text { Width of } \\
\text { Data }\end{array}$ \\
\hline \hline West & $3.14 \pm 0.02 \mathrm{GeV}$ & 1.9 & $3.56 \pm 0.03 \mathrm{GeV}$ & $3.56 \pm 0.08 \mathrm{GeV}$ \\
\hline East & $3.12 \pm 0.02 \mathrm{GeV}$ & 1.9 & $3.64 \pm 0.03 \mathrm{GeV}$ & $3.63 \pm 0.09 \mathrm{GeV}$ \\
\hline
\end{tabular}

\subsection{Systematic Uncertainty on Energy Correc- tions}

The corrections to energy scale and resolution primarily affect the simulated signal acceptance. We determine the errors on these quantities and will use them to quantify their contribution to the systematic error on acceptance.

\subsubsection{Central Electron and Photon Energy Scale Systematic Uncertainty}

In the central region, there is no scaling applied to the energies of simulated electrons and photons. Integrated over all runs, the $Z$ resonance peaks at $(91.18 \pm 0.08)$ $\mathrm{GeV}$, so we need to know how much of an energy scale would be needed in the simulation to fluctuate its peak by the data statistical error of $0.08 \mathrm{GeV}$. Thus, we check what energy scale factors produce $Z$ mass peak at $91.11 \mathrm{GeV}$ and $91.27 \mathrm{GeV}$ in the simulation. We find that scaling the central electron energies by $\pm 0.1 \%$ accomplishes this. Hence, we will apply energy scale factors of 0.999 and 1.001 to the simulation to determine the affect of the energy scale errors on quantities such as acceptance. 


\subsubsection{Central Electron and Photon Energy Resolution Sys- tematic Uncertainty}

For the energy resolution to match the data, we smear the energy of central objects by $3.17 \%$, so that the width of the $Z$ invariant mass resonances matches the width measured in data of $(3.78 \pm 0.09) \mathrm{GeV}$. The statistical error on the data width is $0.09 \mathrm{GeV}$. So, we measure the amount of smearing necessary to produce a $Z$ mass width of $3.69 \mathrm{GeV}$ and $3.87 \mathrm{GeV}(3.78 \mathrm{GeV} \pm 0.09 \mathrm{GeV})$. These results are summarized in Table 5.5. We choose to apply an absolute error of $0.23 \%$ to the smearing correction, half the difference of the minimum and maximum smearing correction.

Table 5.5: Summary of energy smearing applied to central electrons and photons. The energy smearing correction is $\left(3.17_{-0.22}^{+0.24}\right) \%$.

\begin{tabular}{|l|c|c|}
\hline $\begin{array}{l}Z \text { Width } \\
\text { Goal }\end{array}$ & $\begin{array}{c}\text { Smearing } \\
\text { Correction (\%) }\end{array}$ & $\begin{array}{c}\text { Simulated } Z \text { Width } \\
\text { After Smearing }\end{array}$ \\
\hline \hline 3.69 & 2.93 & $3.68 \pm 0.03 \mathrm{GeV}$ \\
\hline 3.78 & 3.17 & $3.77 \pm 0.03 \mathrm{GeV}$ \\
\hline 3.87 & 3.39 & $3.86 \pm 0.03 \mathrm{GeV}$ \\
\hline
\end{tabular}

\subsubsection{Plug EM Object Energy Scale Systematic Uncertainty}

In the data, we scale the energy of west plug EM objects by those factors shown in Tables 5.2 and 5.3. The resulting $Z$ mass peak is $(91.20 \pm 0.08) \mathrm{GeV}$ in the west plug calorimeter and $(91.17 \pm 0.08) \mathrm{GeV}$ in the east. The systematic we apply to the plug energy scale in the simulation is determined by the scale factors necessary to produce a $Z$ mass peak at $91.06 \mathrm{GeV}$ and $91.23 \mathrm{GeV}$, where these values are \pm 0.08 $\mathrm{GeV}$ the central value in the simulation of $91.13 \mathrm{GeV}$. We find that a scale factor 
of $\pm 0.2 \%$ fluctuates the simulated peak appropriately. Hence, we will fluctuate the energy scale of the simulation by 0.998 and 1.002 to measure the effect of the scale uncertainty on such quantities as signal acceptance. This uncertainty is the same for west plug objects as it is for east plug objects.

\subsubsection{Plug EM Object Resolution Systematic Uncertainty}

The energy resolution of simulated plug EM objects was better than those in the data. To match the simulated resolution to that of the data, the energies of simulated plug EM objects are smeared by 1.9\%.Again, we use the statistical error on the width of the data central-plug $Z \rightarrow e e$ peak to determine the error on these smear factors. The error on the width in the data is $0.08 \mathrm{GeV}$ in the west and 0.09 in the east. Thus, we float the smearing factors in the west and east regions such that the simulated width fluctuates up and down by $0.08 \mathrm{GeV}$ and $0.09 \mathrm{GeV}$, respectively, from their central value. The errors are summarized in Tables 5.6 and 5.7. An absolute error of $0.65 \%$ is assigned to the west plug EM object energy smear factor. For the east plug EM object smear factor, $0.8 \%$ is assigned as the absolute error. These errors are approximately half the difference of the minimum and maximum smear factors.

Table 5.6: Summary of energy smearing applied to west plug EM objects. The energy smearing correction is $\left(1.9_{-0.7}^{+0.6}\right) \%$.

\begin{tabular}{|l|c|c|}
\hline $\begin{array}{l}Z \text { Width } \\
\text { Goal }\end{array}$ & $\begin{array}{c}\text { Smearing (\%) } \\
\text { Correction }\end{array}$ & $\begin{array}{c}\text { Simulated } Z \text { Width } \\
\text { After Smearing }\end{array}$ \\
\hline \hline 3.48 & 1.2 & $3.47 \pm 0.03 \mathrm{GeV}$ \\
\hline 3.56 & 1.9 & $3.56 \pm 0.03 \mathrm{GeV}$ \\
\hline 3.64 & 2.5 & $3.64 \pm 0.03 \mathrm{GeV}$ \\
\hline
\end{tabular}


Table 5.7: Summary of energy smearing applied to east plug EM objects. The energy smearing correction is $\left(1.9_{-0.9}^{+0.7}\right) \%$.

\begin{tabular}{|l|c|c|}
\hline $\begin{array}{l}Z \text { Width } \\
\text { Goal }\end{array}$ & $\begin{array}{c}\text { Smearing (\%) } \\
\text { Correction }\end{array}$ & $\begin{array}{c}\text { Simulated } Z \text { Width } \\
\text { After Smearing }\end{array}$ \\
\hline \hline 3.54 & 1.0 & $3.55 \pm 0.03 \mathrm{GeV}$ \\
\hline 3.63 & 1.9 & $3.64 \pm 0.03 \mathrm{GeV}$ \\
\hline 3.72 & 2.6 & $3.73 \pm 0.03 \mathrm{GeV}$ \\
\hline
\end{tabular}

\subsection{Summary}

Because the energies of central and plug EM objects are not properly calibrated in the data, we measure correction factors which scale the energies to agree with the well-known $Z$ boson mass. Because the energy response of the detector changes with time, run-dependent corrections are applied to the energies. These are summarized for central electrons and photons in Table 5.1. Tables 5.2 and 5.3 summarize the run-dependent energy scale factors applied to west and east plug EM objects.

The error on the scale of simulated energies for central electrons and photons is estimated to be $0.1 \%$. The energy scale of simulated plug EM objects is determined to have a $0.2 \%$ error. We will study the effect of these errors in section 7.3.8.

The resolution of the calorimeter is underestimated in the simulation. To account for this, we smear the energies of simulated central electrons and photons by $(3.17 \pm 0.23) \%$, west plug EM objects by $(1.90 \pm 0.65) \%$, and east plug EM objects $(1.90 \pm 0.80) \%$. We discuss the effect of the error on the smear factors in Section 7.3.9. 


\section{Chapter 6}

\section{Efficiency}

In this analysis, we search for events with two electrons and a photon, where at least one electron is central. However, several issues such as object identification, trigger, and $Z_{v t x}$ requirements result in a loss of dielectron + photon candidates. In this chapter we discuss the measurements of efficiencies for the identification of central electrons, central photons and plug EM objects and also the trigger and $Z_{v t x}$ efficiencies.

\subsection{Identification Efficiencies}

Identification efficiency is defined as the rate at which real, prompt electrons, photons, or plug EM objects are identified as such after selection criteria are applied. To study these identification efficiencies, we need pure samples of events containing these objects. $Z \rightarrow e e$ events provide a source of easily identifiable electrons and plug EM objects with minimal background contamination. There is no corresponding source of pure photons, thus electrons from $Z \rightarrow e e$ events are used to the "emulate" photons. We measure and compare the identification efficiencies in data and simulated $Z \rightarrow e e$ Monte Carlo. 


\subsubsection{Central Electron Identification Efficiency}

\section{Measurement}

The central electron identification efficiency is measured using $Z \rightarrow e e$ events where both electrons are central. We define a base sample that consists of two EM objects that pass the following criteria:

- central

- fiducial

- $E_{T}>25 \mathrm{GeV}$

- an associated track with $p_{T}>10 \mathrm{GeV}$

- $\left|Z_{\circ}\right|<60 \mathrm{~cm}$

The invariant mass of the pair of electron candidates must lie in the range of the $Z$ boson mass, $81<M_{e e}<101 \mathrm{GeV}$. We count the number of events for which both candidates pass the central electron selection criteria (see Section 4.2), and the number of events in which one candidate electron passes the selection criteria and one fails at least one identification requirement.

This sample of $Z \rightarrow e e$ candidates contains some background contamination due to QCD events. A jet that fakes an electron is equally likely to be positively charge as it is to be negatively charged. So, we use a "like-sign/unlike-sign" method to subtract background from the measurement. Four categories of events are defined:

- $N_{p p \text { us }}^{\text {raw }}$ is the number of unlike-signed events for which both electrons pass the selection cuts 
- $N_{p p l s}^{r a w}$ is the number of like-signed events for which both electrons pass the selection cuts

- $N_{p f u s}^{r a w}$ is the number of unlike-signed events for which one electron passes and one fails the selection cuts

- $N_{p f \text { us }}^{r a w}$ is the number of like-signed events for which one electron passes and one fails the selection cuts

Then, to remove the QCD background, we subtract the number of like-signed events from the number of unlike-signed events to obtain the number of real $Z \rightarrow e e$ passpass $\left(N_{p p}\right)$ and pass-fail $\left(N_{p f}\right)$ events:

$$
\begin{aligned}
& N_{p p}=N_{p p u s}^{r a w}-N_{p p l s}^{r a w} \\
& N_{p f}=N_{p f u s}^{\text {raw }}-N_{p f l s}^{\text {raw }}
\end{aligned}
$$

The background subtracted efficiency $\left(\epsilon_{e}\right)$ is then defined by the following relation:

$$
\epsilon_{e}=\frac{2 N_{p p}}{2 N_{p p}+N_{p f}}
$$

From $Z \rightarrow e e$ data, we measure $\epsilon_{e}=0.940 \pm 0.003$ (stat).

We validate the simulation by performing the same measurement using the standard sample of PYTHIA+CdfSim $Z \rightarrow e e$ events used by the electroweak $Z \rightarrow e e$ working group, the "zewk1e" sample. In the simulation we measure $\epsilon_{e}=0.927 \pm 0.001($ stat $)$, which is fairly close to the result from data. 


\section{$N-1$ Identification Efficiency}

$N-1$ identification efficiency is defined as the efficiency of cut $N$, after all other cuts have been applied. This is measured by defining the base sample such that it contains two electron candidates that pass all cuts except the $N$ th cut (ie. the $N$ th cut is not tested as part of the base sample). Again, we apply the $M_{e e}$ cut and follow the like-sign/unlike-sign procedure for background subtraction. Then, $N_{p f}$ simply becomes the background subtracted number of events in which one electron passes the $N$ th cut and the second fails. Likewise, $N_{p p}$ denotes the background subtracted number of events for which both electrons pass all the selection cuts. Equation 6.2 can then be used to define the $N-1$ identification efficiency. In Table 6.1, we compare the $N-1$ identification efficiencies of each cut between data and simulation. Overall, there is good data-simulation agreement, but there is some difference in $L_{s h r}$ which is due to a known problem in the simulation that is fixed in later versions of the software.

\begin{tabular}{|l|c|c|}
\hline Variable & Data $N-1 \epsilon_{e}$ & Simulation $N-1 \epsilon_{e}$ \\
\hline$L_{\text {shr }}$ & $0.994 \pm 0.001$ & $0.9843 \pm 0.0005$ \\
\hline$\frac{E_{H a d}}{E_{E M}}$ & $0.992 \pm 0.001$ & $0.9889 \pm 0.0004$ \\
\hline Cal Iso & $0.976 \pm 0.002$ & $0.9778 \pm 0.0006$ \\
\hline$\frac{E}{p}$ & $0.994 \pm 0.001$ & $0.9956 \pm 0.0003$ \\
\hline$\Delta X$ & $0.981 \pm 0.002$ & $0.9810 \pm 0.0006$ \\
\hline$\Delta Z$ & $0.997 \pm 0.001$ & $0.9989 \pm 0.0001$ \\
\hline
\end{tabular}

Table 6.1: $N-1$ central electron identification efficiency. The errors shown are statistical.

\section{$N-1$ Efficiency Distributions}

Figures 6.1 show the $N-1$ distributions for the selection criteria in data and simulation. The distributions show good agreement between data and simulation, 
with the exception of $L_{s h r}$.

Figure 6.2 shows the efficiency as a function of $\eta_{d}, \phi, E_{T}$, and run number. The efficiency is steady with time.

The differences between data and simulation in the $\eta_{d}$ and $E_{T}$ distributions are explained by the $L_{s h r}$ simulation problem. This problem is exacerbated in the west calorimeter, as shown in the plot of $\eta_{d}$. Also, the difference in the $E_{T}$ distribution is due to the $L_{s h r}$ cut. As shown in Figure 6.3, this is confirmed by removing the $L_{s h r}$ requirement and finding that the $\eta_{d}$ and $E_{T}$ efficiency distributions agree nicely.

The difference between data and simulation in the efficiency vs $E_{T}$ distribution increases with $E_{T}$. Since we are searching for high energy electrons, this would pose a problem. However, the $L_{s h r}$ cut is removed at $E_{T}=100 \mathrm{GeV}$, protecting us from the effect of the $L_{s h r}$ cut at high $E_{T}$.

\section{Summary}

We conclude that the simulation adequately describes the electron identification variables. We quote a $1 \%$ systematic uncertainty on the electron selection efficiency due to differences between the simulation and data.

\subsubsection{Central Photon Identification Efficiency}

\section{Measurement}

$Z \rightarrow e e$ events provide a clean source of high $E_{T}$ electrons. Unfortunately, there is no corresponding source of high $E_{T}$ photons from which to measure the photon identification efficiency. Unlike electrons, photons leave no tracks in the tracking detector, but they do exhibit behavior in the calorimeter that is similar to electrons. Thus, electrons from $Z \rightarrow e e$ events are used to emulate the photon cuts and 


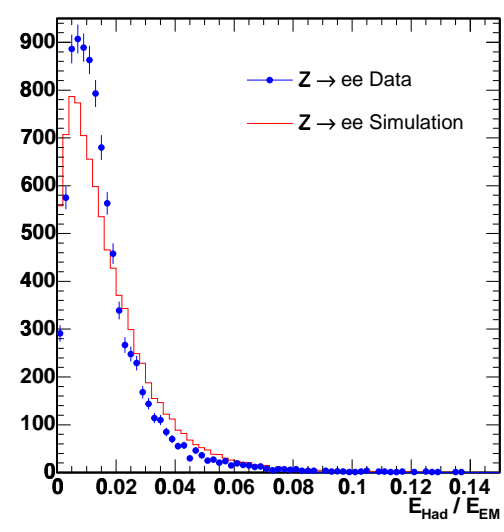

(a)

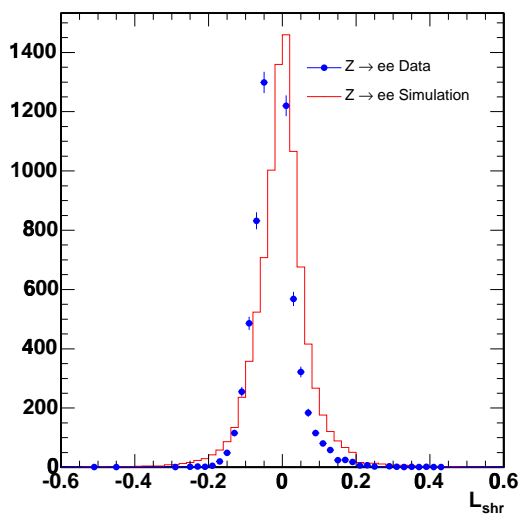

(c)

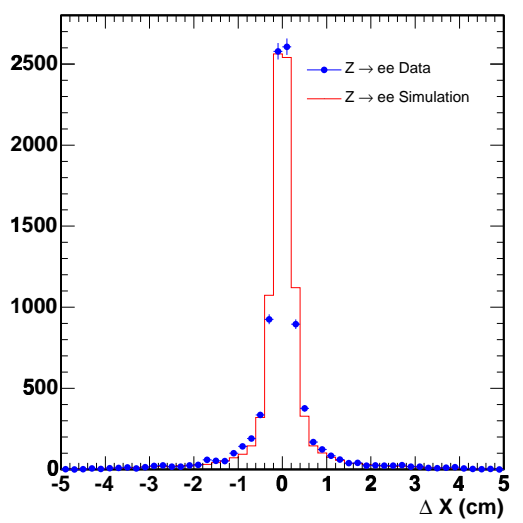

(e)

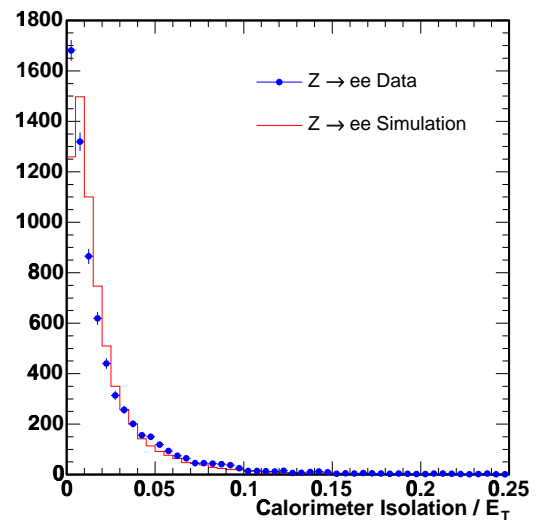

(b)

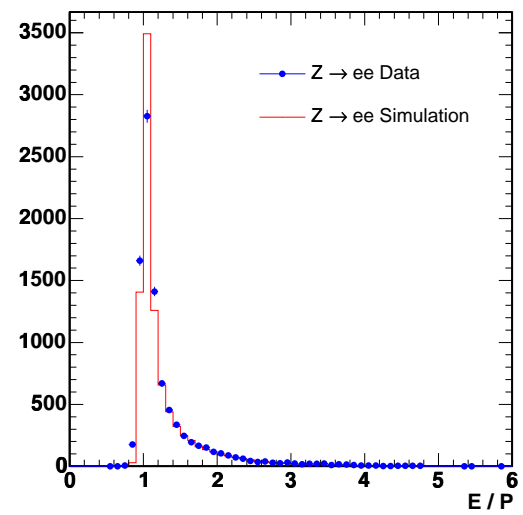

(d)

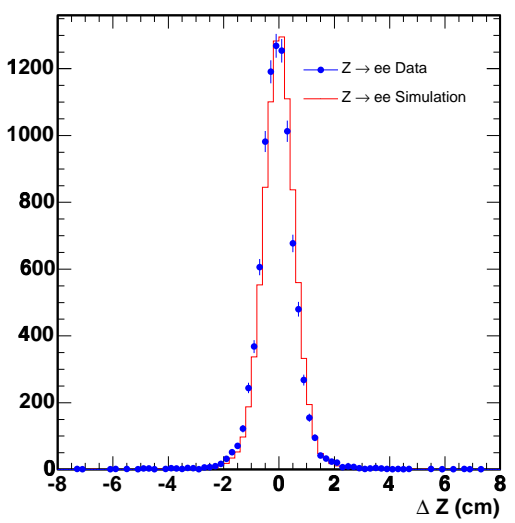

(f)

Figure 6.1: $N-1$ Electron identification distributions. 


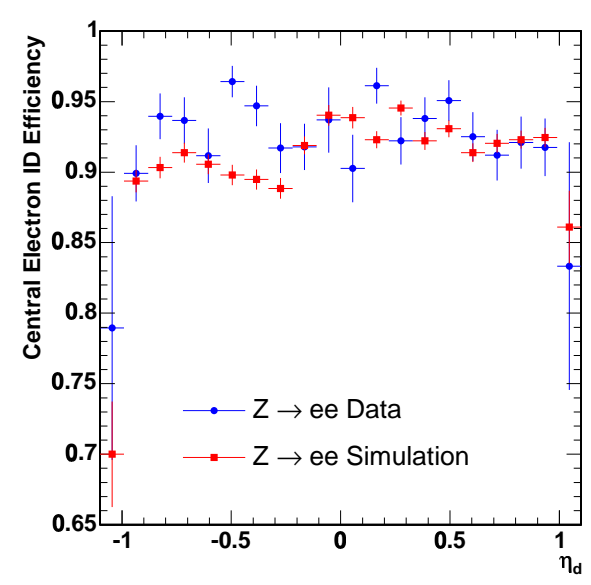

(a)

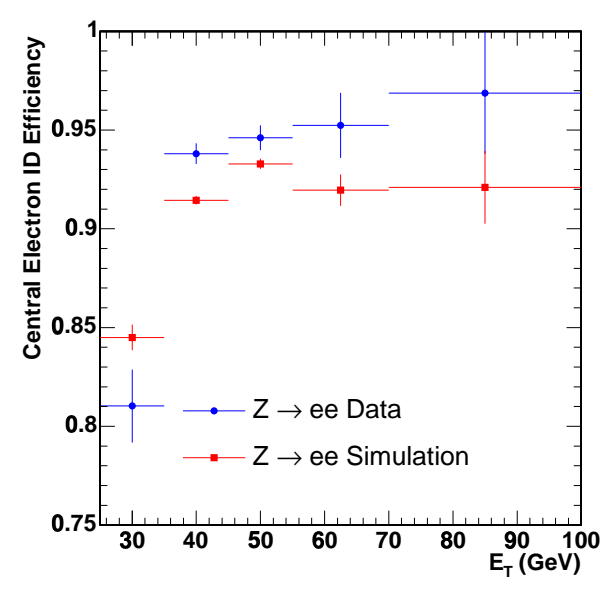

(c)

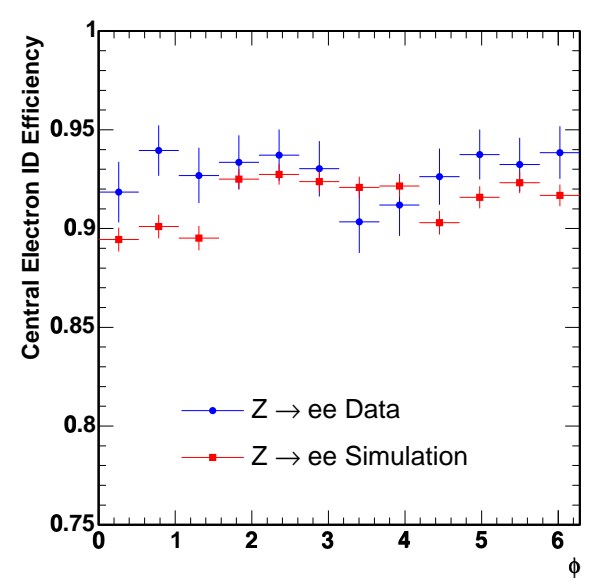

(b)

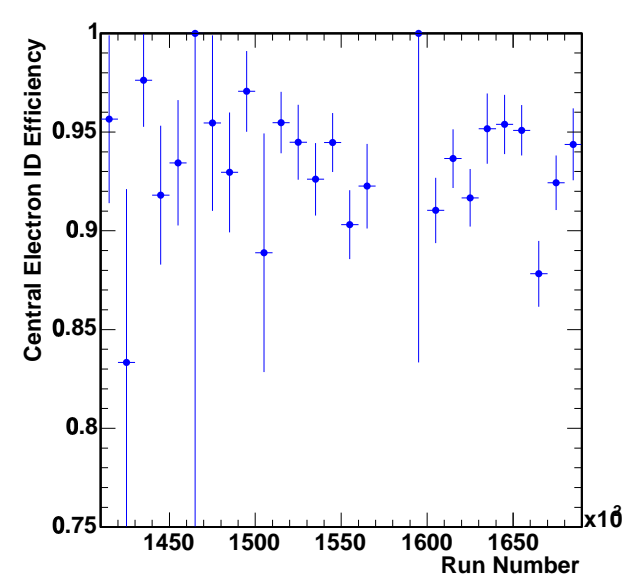

(d)

Figure 6.2: Electron identification efficiency as a function of $\eta_{d}, \phi, E_{T}$, and run number. 


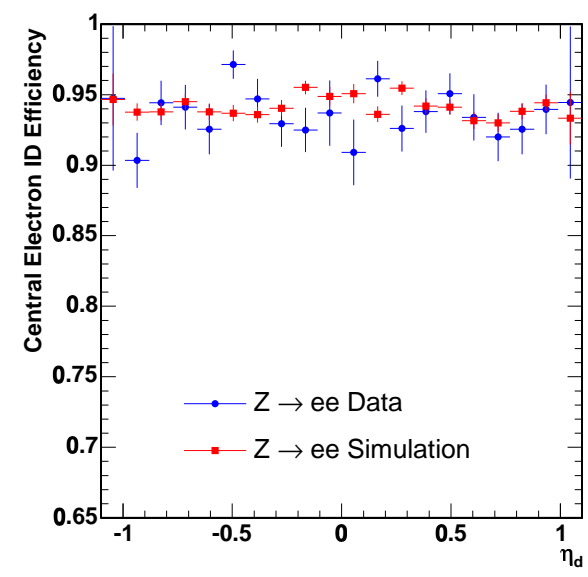

(a)

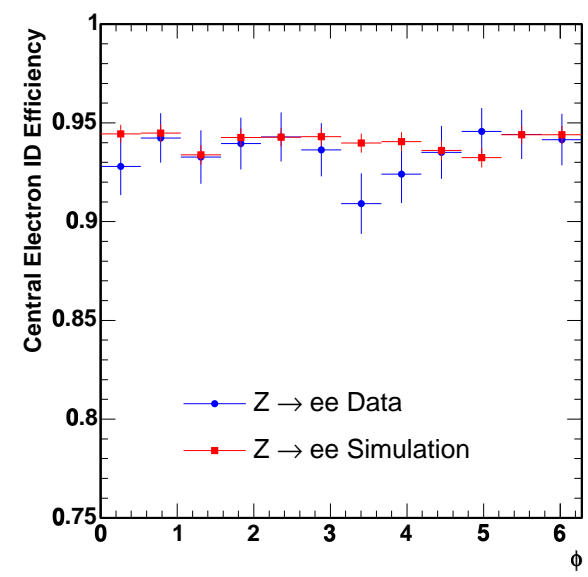

(b)

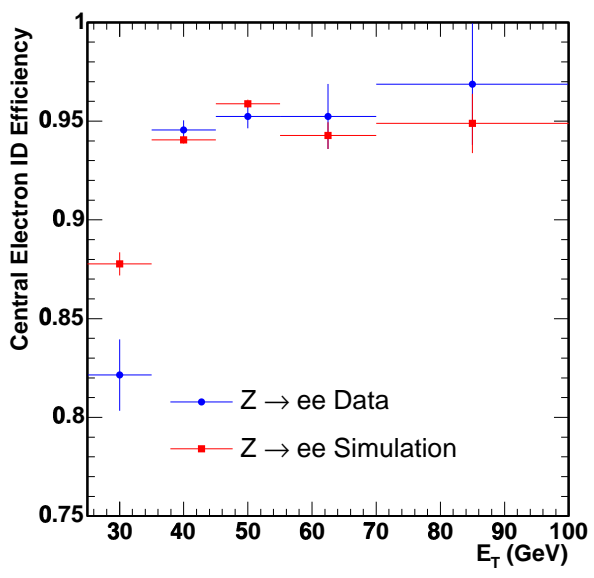

(c)

Figure 6.3: Electron identification efficiency as a function of $\eta_{d}, \phi$, and $E_{T}$ with no $L_{s h r}$ cuts. 
determine the efficiency. The results from data are compared to the simulation to validate the simulation's treatment of the photon variables.

We start by creating a base sample of events. The first step is to select events with two central, fiducial EM objects with $E_{T}>25 \mathrm{GeV}$ and track $p_{T}>10 \mathrm{GeV}$ and two-body invariant mass between 81 and $101 \mathrm{GeV}$. Then, one of the two EM objects is randomly chosen and required to pass a set of extra tight electron cuts listed in Table 6.2. These cuts are similar to those used by the electroweak group for tight electron selection. Events passing these requirements comprise the base sample, which is a clean sample of $Z \rightarrow e e$ events.

Now, we emulate the central photon cuts in Table 4.3 and check how often the second electron passes them. To emulate these cuts, the track associated with the electron must be taken into account. In particular, for the N3D cut, we require the number of tracks be less than or equal to 2. If a second track exists, it must have $p_{T}<1 \mathrm{GeV}+0.005 \cdot \mathrm{E}_{\mathrm{T}}$. Similarly, for tracking isolation, the non-beam constrained $p_{T}$ of the track associated with the electron is subtracted from the value of the tracking isolation. All the other cuts are applied to the electron as shown in Table 4.3. Then, the photon identification efficiency $\left(\epsilon_{\gamma}\right)$ is defined as:

$$
\epsilon_{\gamma}=\frac{\text { number of events that pass emulated photon cuts }}{\text { number of events in base sample }}
$$

Background subtraction is performed on these samples using a like-sign/unlikesign method similar to the one used for the central electron identification efficiency measurement.

The procedure is repeated with simulated $Z \rightarrow e e$ Monte Carlo events and compared to data, the results of which are in Table 6.3. The "emulated photon" efficiency after all cuts is $(75.5 \pm 0.7($ stat $)) \%$ in the data and $(78.3 \pm 0.2($ stat $)) \%$ in 


\begin{tabular}{|l|c|}
\hline Variable & cut \\
\hline \hline$\eta$ & central \\
\hline$E_{T}$ & $>25 \mathrm{GeV}$ \\
\hline Track $p_{T}$ & $>10 \mathrm{GeV}$ \\
\hline Total Iso $(\Delta R<0.4)$ & $<4 \mathrm{GeV}$ \\
\hline$\left|z_{0}\right|$ & $<60 \mathrm{~cm}$ \\
\hline$\frac{E_{H a d}}{E_{E M}}$ & $<0.055+0.00045 \cdot E$ \\
\hline$\frac{E}{p}\left(E_{T}<50 \mathrm{GeV}\right)$ & $<2.0$ \\
\hline$L_{s h r}$ & $<0.2$ \\
\hline charge-signed $\Delta X$ & $-3 .<Q_{t r k} \cdot \Delta X<1.5 \mathrm{~cm}$ \\
\hline$|\Delta Z|$ & $<3 \mathrm{~cm}$ \\
\hline$\chi_{\text {strip }}^{2}$ & $<10$. \\
\hline$\#$ of Axial Layers with 6 hits & $>=3$ \\
\hline$\#$ of Stereo Hits with 6 hits & $>=3$ \\
\hline
\end{tabular}

Table 6.2: Extra tight electron selection criteria used in the central photon and plug EM object identification studies.

the simulation.

\section{Effect of Bremsstrahlung}

Table 6.3 shows the photon ID efficiency cuts as they are applied sequentially. The emulated photon ID efficiency is low due to the $\chi^{2}$ cut. This is partially because we are using electrons, which radiate photons and thereby lower the efficiency of the $\chi_{\text {wire }}^{2}$ cut (in the bend view). The effect of bremsstrahlung is investigated by making an $0.9<E / p<1.1$ cut after the second CES Strip/Wire energy selection requirement and before the $\frac{E_{H a d}}{E_{E M}}$ cut. We use the relative efficiency of the average $\chi^{2}$ and $\frac{E_{H a d}}{E_{E M}}$ cuts to estimate the "emulated" efficiency without bremsstrahlung $\left(\epsilon_{\gamma}^{\text {no brem }}\right)$ using the following equation:

$$
\epsilon_{\gamma}^{\text {no brem }}=\frac{\text { events passing average } \chi^{2} \text { cut with E/p Cut }}{\text { events passing } \frac{\mathrm{E}_{\mathrm{Had}}}{\mathrm{E}_{\mathrm{EM}}} \text { cut with } \mathrm{E} / \mathrm{p} \mathrm{Cut}} \cdot \frac{\mathrm{E}_{\mathrm{Had}}}{\mathrm{E}_{\mathrm{EM}}} \text { cut }
$$


Using this relation, the total photon efficiency is measured at $(84.6 \pm 0.7($ stat $)) \%$ in the data and $(86.7 \pm 0.2($ stat $)) \%$ in the simulation. We conclude that the simulation gives a good description of the photon identification variables and can be used to predict the photon efficiency with an uncertainty of $3 \%$.

\begin{tabular}{|l|c|c|c|c|c|c|}
\hline & \multicolumn{3}{|c|}{ Data } & \multicolumn{3}{|c|}{$Z \rightarrow e e$ Simulation } \\
Variable & Number & $\begin{array}{c}\text { Rel } \\
\text { Events }\end{array}$ & $\begin{array}{c}\text { Cumulative } \\
\epsilon_{\gamma}(\%)\end{array}$ & $\begin{array}{c}\text { Number } \\
\epsilon_{\gamma}(\%)\end{array}$ & $\begin{array}{c}\text { Rel } \\
\text { Events }\end{array}$ & $\begin{array}{c}\text { Cumulative } \\
\epsilon_{\gamma}(\%)\end{array}$ \\
$\epsilon_{\gamma}(\%)$
\end{tabular}

Table 6.3: Sequential efficiencies of photon cuts. Each cut is applied in addition to the previous cuts. The last line has the cumulative efficiency for electrons that behave most like photons. The errors shown are statistical.

\section{$N-1$ Identification Efficiency}

Table 6.4 shows the data-simulation comparison of the $N-1$ efficiencies for the "emulated" photon cuts, indicating good agreement. The $N-1$ efficiency is defined as the efficiency of a cut after all other cuts have been applied. The base sample used in the denominator of Equation 6.3 is the number of events for which the "emulated" 
photon passes all the selection criteria except the $N$ th cut. The numerator of Equation 6.3 is the number of events in the base sample which pass the $N$ th cut. The $N-1$ cut efficiencies are shown in Tables 6.4 and 6.5 for electrons without and with the tight $E / p$ cut applied, respectively.

\begin{tabular}{|l|c|c|}
\hline Variable & Data $N-1 \epsilon_{\gamma}$ & Simulation $N-1 \epsilon_{\gamma}$ \\
\hline \hline Calorimeter Iso $(\Delta R<0.4)$ & $0.989 \pm 0.002$ & $0.988 \pm 0.001$ \\
\hline Track Iso $(\Delta R<0.4)$ & $0.983 \pm 0.003$ & $0.987 \pm 0.001$ \\
\hline $\mathrm{N} 3 \mathrm{~d}$ Tracks & $0.962 \pm 0.003$ & $0.973 \pm 0.001$ \\
\hline$\frac{E_{H a d}}{E_{E M}}$ & $0.9997 \pm 0.0003$ & $0.9994 \pm 0.0001$ \\
\hline Average $\chi^{2}$ & $0.876 \pm 0.006$ & $0.901 \pm 0.002$ \\
\hline 2nd CES Energy & $0.976 \pm 0.003$ & $0.968 \pm 0.001$ \\
\hline
\end{tabular}

Table 6.4: $N-1$ photon ID efficiency without the tight $E / p$ cut. The errors shown are statistical.

\begin{tabular}{|l|c|c|}
\hline Variable & Data $N-1 \epsilon_{\gamma}$ & Simulation $N-1 \epsilon_{\gamma}$ \\
\hline \hline Calorimeter Iso $(\Delta R<0.4)$ & $0.990 \pm 0.002$ & $0.988 \pm 0.001$ \\
\hline Track Iso $(\Delta R<0.4)$ & $0.990 \pm 0.002$ & $0.996 \pm 0.001$ \\
\hline N3d Tracks & $0.976 \pm 0.004$ & $0.978 \pm 0.001$ \\
\hline$\frac{E_{H a d}}{E_{E M}}$ & $0.999 \pm 0.001$ & $0.9994 \pm 0.0002$ \\
\hline Average $\chi^{2}$ & $0.982 \pm 0.003$ & $0.9984 \pm 0.0003$ \\
\hline 2nd CES Energy & $0.979 \pm 0.003$ & $0.979 \pm 0.001$ \\
\hline
\end{tabular}

Table 6.5: $N-1$ photon ID efficiency with the tight $E / p$ cut. The errors shown are statistical.

\section{"Emulated" $N-1$ Identification Distributions}

Figure 6.4 shows the "emulated" $N-1$ distributions for the photon identification variables in $Z \rightarrow e e$ data compared to $Z \rightarrow e e$ simulation without the $E / p$ cut. Figure 6.5 shows the distributions with the tight $E / p$ cut. These show good agreement between data and simulation. 


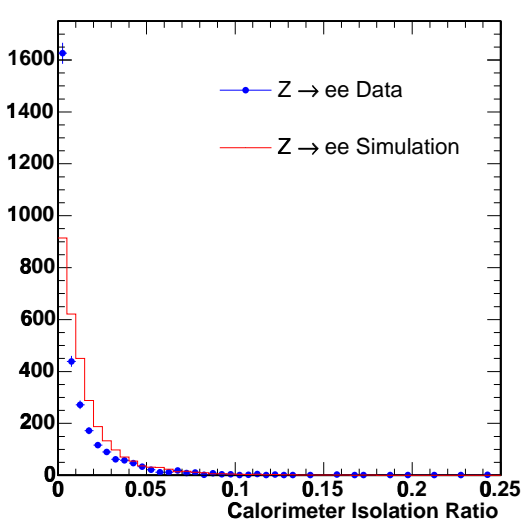

(a)

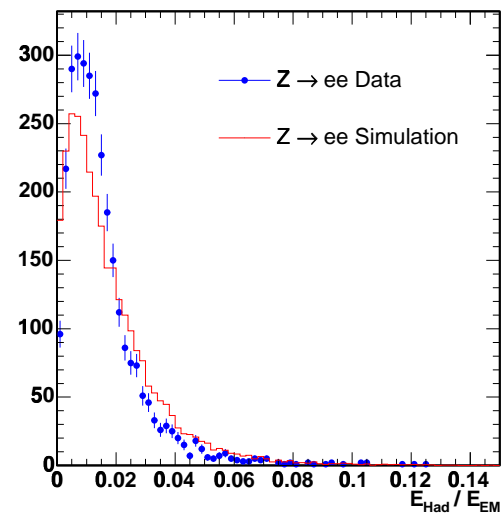

(c)

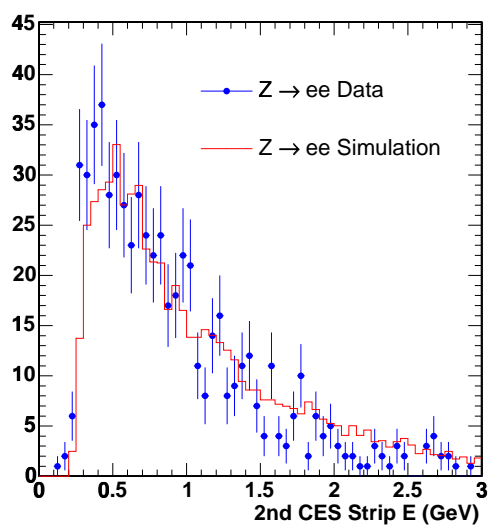

(e)

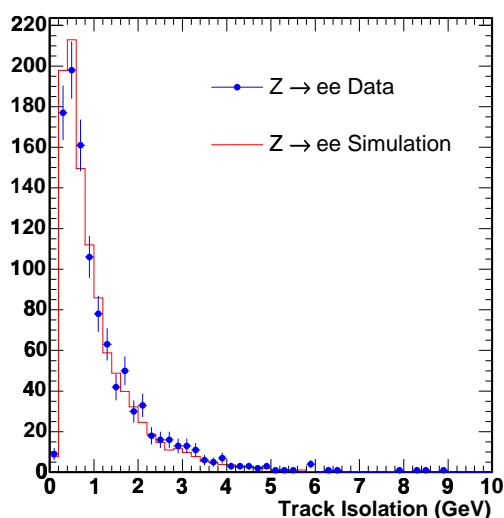

(b)

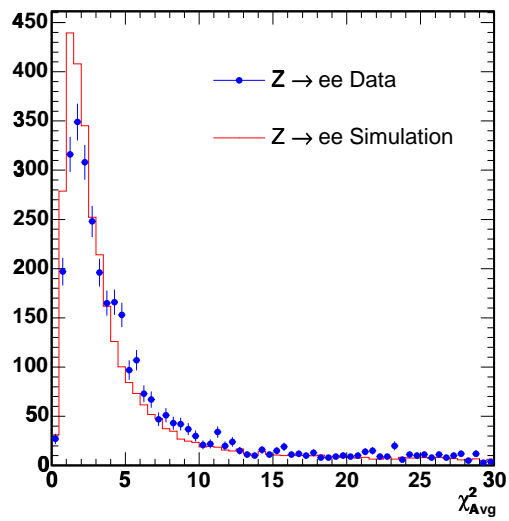

(d)

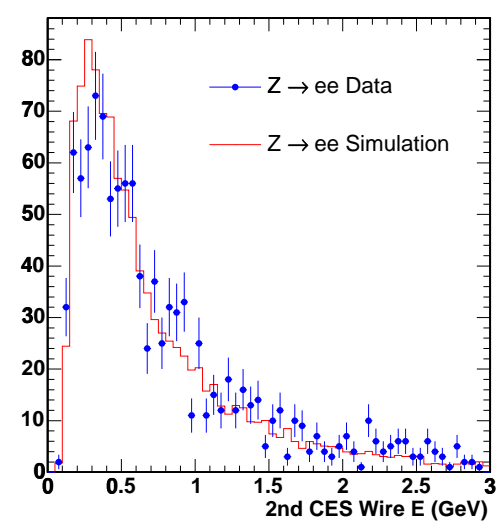

(f)

Figure 6.4: Emulated $N-1$ Photon Identification Distributions without $E / p$ cut. 


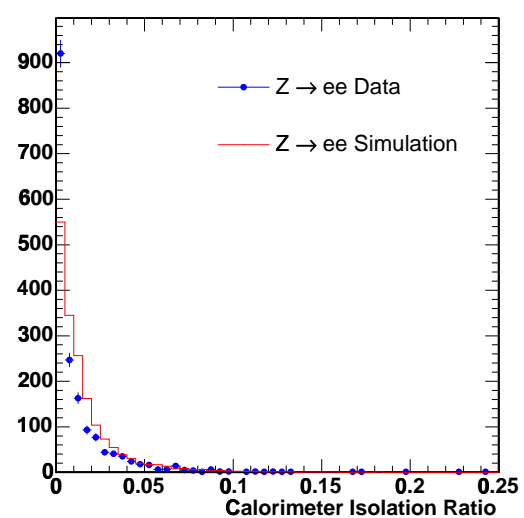

(a)

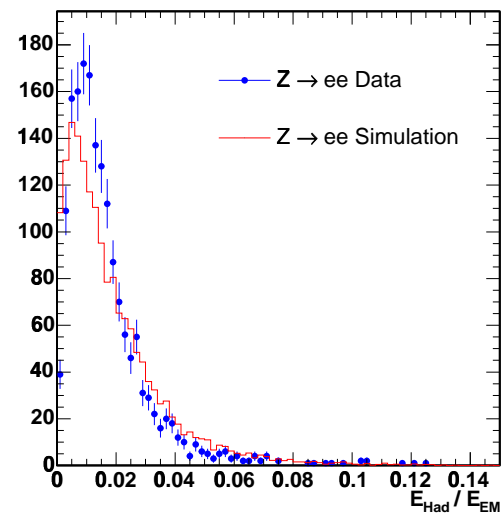

(c)

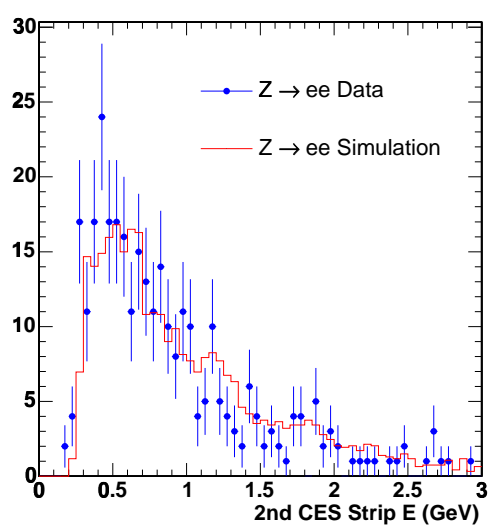

(e)

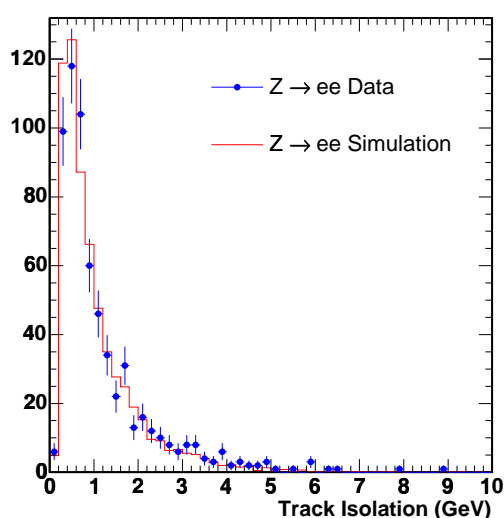

(b)

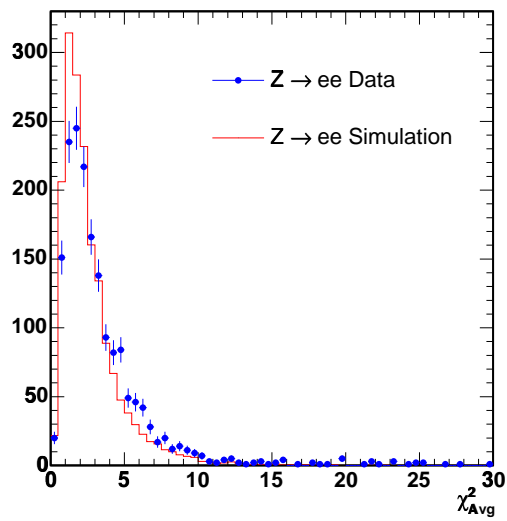

(d)

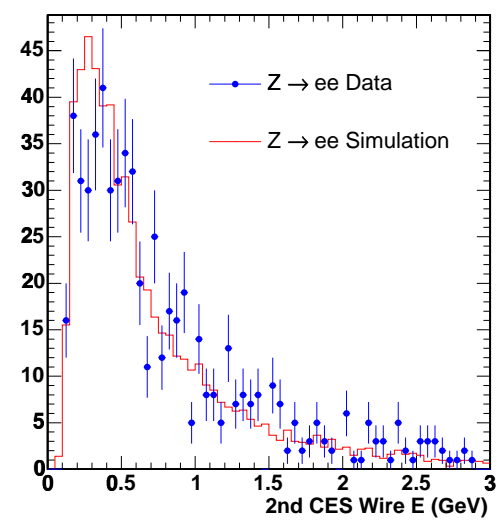

(f)

Figure 6.5: Emulated $N-1$ Photon Identification Distributions with $E / p$ cut. 
Figure 6.6 shows the emulated photon ID efficiency with no $E / p$ requirement as a function of $\eta_{d}, \phi, E_{T}$, and run number. The data efficiency is stable in time. The $\eta_{d}, \phi$, and $E_{T}$ dependencies are similar between data and simulation.

\section{Summary}

We conclude that the simulation adequately describes the photon identification variables. A 3\% systematic error on the photon selection efficiency is quoted.

\subsubsection{Plug EM Object Identification Efficiency}

\subsubsection{Measurement}

Central-plug $Z \rightarrow e e$ data is used to study the efficiency of the plug identification cuts. The base sample is comprised of events with one central electron that passes the extra tight electron cuts in Table 6.2. Additionally, the event must have a plug, fiducial EM object with $E_{T}>25 \mathrm{GeV}$. The invariant mass of the central electronplug EM object pair must lie within the range of the $Z$ boson mass, $81-101 \mathrm{GeV}$. The plug EM object identification efficiency $\left(\epsilon_{p}\right)$ is defined as the fraction of events in the base sample for which the plug EM object passes the identification cuts:

$$
\epsilon_{\mathrm{p}}=\frac{\text { number of events that pass PEM cuts }}{\text { number of events in base sample }}
$$

The plug EM object efficiency in data is $0.856 \pm 0.004$ (stat). However, the sample contains background contamination. If the $M_{e e}$ cut is tightened to between $86 \mathrm{GeV}$ and $96 \mathrm{GeV}$, effectively removing half of the background, the efficiency increases to $(87.4 \pm 0.4($ stat $)) \%$. The efficiency increases to $(88.5 \pm 0.6($ stat $)) \%$ when the 


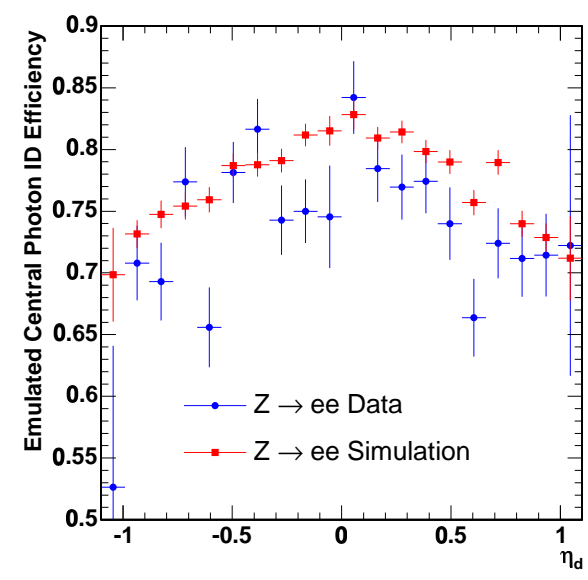

(a)

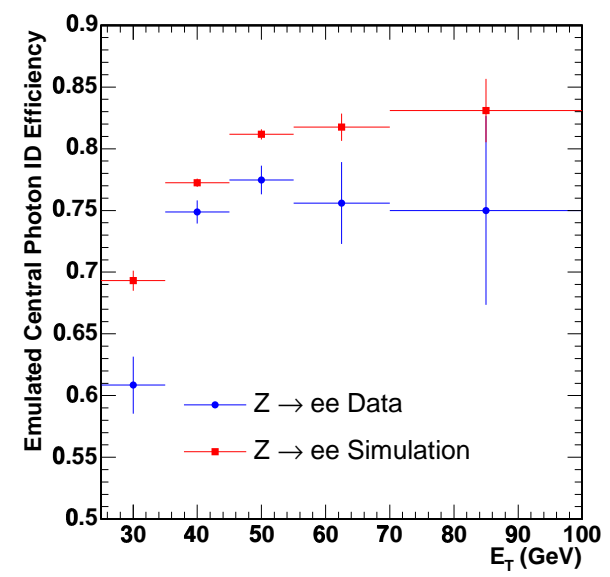

(c)

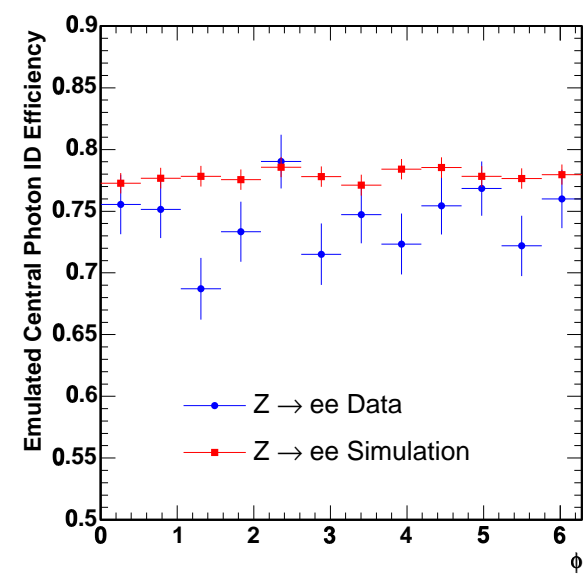

(b)

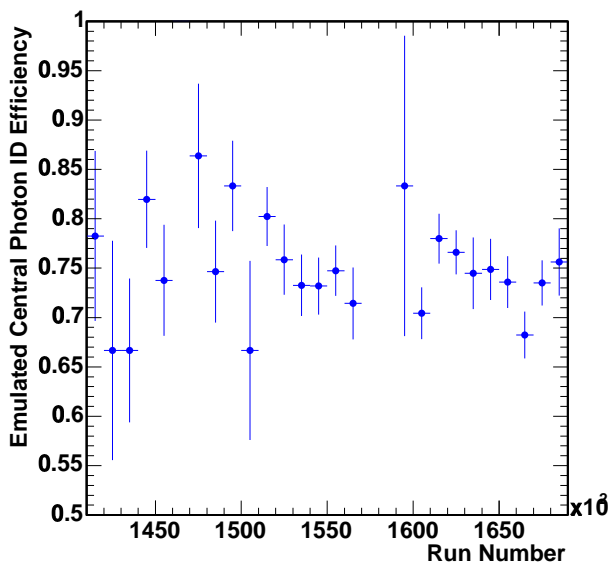

(d)

Figure 6.6: Photon identification efficiency as a function of $\eta_{d}, \phi, E_{T}$, and run number with no $E / p$ cut. 
background is reduced by another factor of two by tightening the isolation ratio cut from $10 \%$ to $5 \%$. Extrapolating these increases in efficiency to zero background, the plug EM object ID efficiency is $(89.0 \pm 0.6($ stat $)) \%$.

The data are compared to simulation by measuring the efficiency of plug EM objects in the "zewk1e" Monte Carlo sample. The simulated efficiency is (93.4 \pm $0.1($ stat $)) \%$. In Table 6.6 and Figures $6.7(\mathrm{a}-\mathrm{c})$, the $N-1$ ID cut efficiencies and distributions are compared between data and simulation. The data-simulation difference comes primarily from the PEM $\chi^{2}$.

\begin{tabular}{|l|c|c|}
\hline Variable & Data $N-1 \epsilon_{p}(\%)$ & Simulation $N-1 \epsilon_{p}(\%)$ \\
\hline \hline$\frac{I s o(\Delta R<0.4)}{E_{T}}$ & $99.2 \pm 0.1$ & $99.3 \pm 0.1$ \\
\hline$\frac{E_{H a d}}{E_{E M}}$ & $99.9 \pm 0.1$ & $99.99 \pm 0.01$ \\
\hline PEM $\chi^{2}$ & $91.3 \pm 0.4$ & $94.5 \pm 0.1$ \\
\hline
\end{tabular}

Table 6.6: $N-1$ Plug EM Object Identification Efficiency using $Z \rightarrow e e$ centralplug events.

In order to correctly calculate the total signal acceptance of the excited electron models, the data-simulation identification efficiency differences need to be understood.

The data-simulation efficiency difference is likely due to extra energy in the plug detector that is not included in the simulation. We study the effect of adding extra minimum-bias interactions to the $Z \rightarrow e e$ Monte Carlo. The number of extra minimum-bias interactions per event is determined by a Poisson distribution with a mean of 1 interaction per event. The resulting efficiency prediction is $(90.0 \pm$ $0.6(s t a t)) \%$. This is in good agreement with the projected plug EM object ID efficiency of $89 \%$ for $Z \rightarrow e e$ data with no background contamination. The $N-1$ ID efficiencies with extra interactions are shown in Table 6.7. The PEM $\chi^{2}$ distribution 

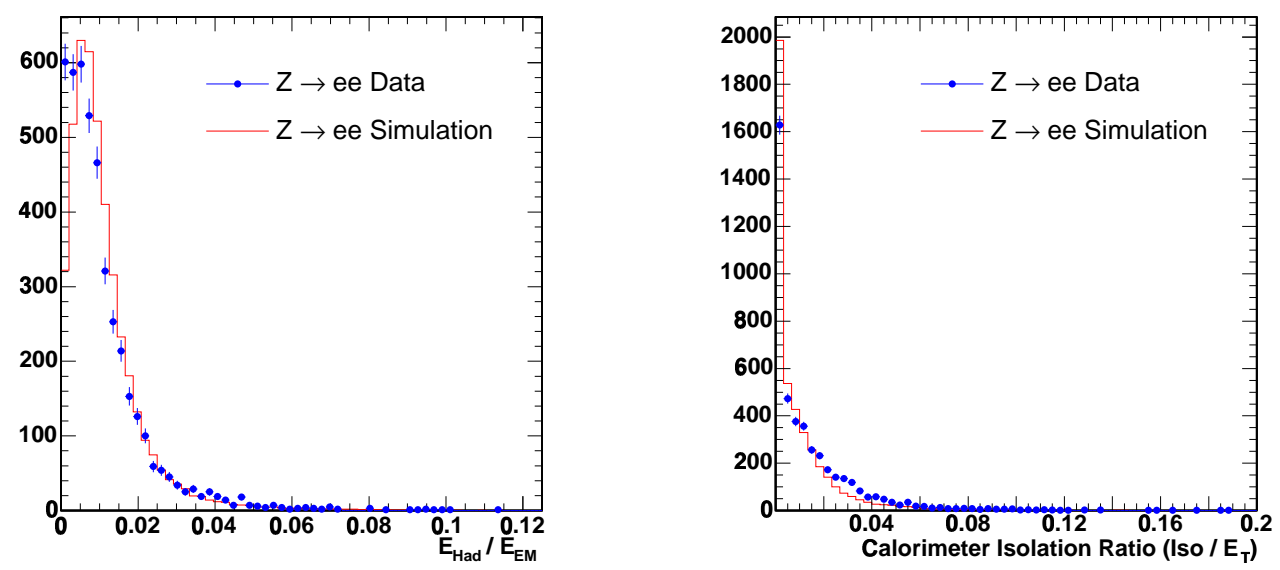

(a)

(b)

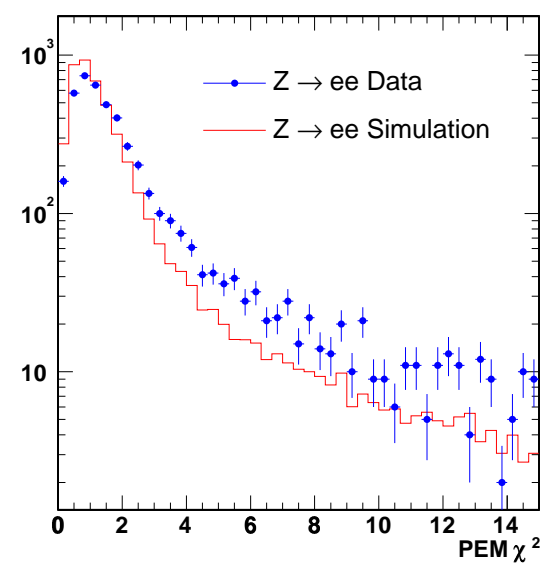

(c)

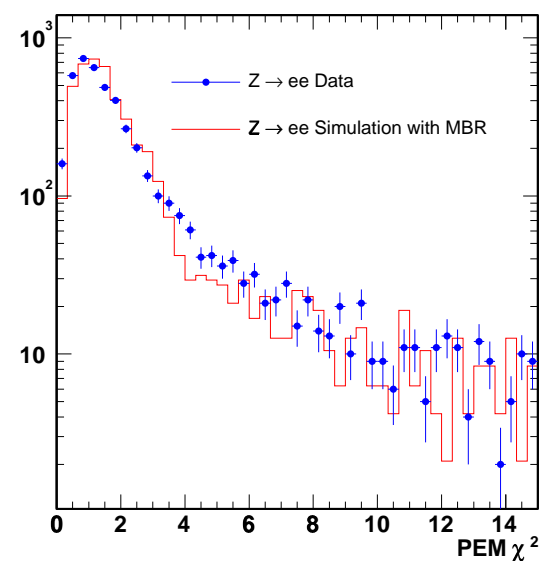

(d)

Figure 6.7: $N-1$ Plug EM object identification distributions. Figures (a)-(c) have no extra interactions. Figure $(d)$ has a mean of 1 extra minimum-bias interaction per event. 
is the one affected by the extra interactions. This $N-1$ ID distribution is shown in Figure 6.7(d).

\begin{tabular}{|l|c|c|c|}
\hline Variable & Data $N-1 \epsilon_{p}$ & $\begin{array}{c}\text { Simulation } N-1 \epsilon \\
\text { Default }\end{array}$ & $\begin{array}{c}\text { Simulation } N-1 \epsilon_{p} \\
\text { Extra Interactions }\end{array}$ \\
\hline \hline Calorimeter Iso & $0.992 \pm 0.001$ & $0.9931 \pm 0.0004$ & $0.993 \pm 0.002$ \\
\hline$\frac{E_{H a d}}{E_{E M}}$ & $0.999 \pm 0.001$ & $0.9991 \pm 0.0001$ & $0.999 \pm 0.001$ \\
\hline PEM $\chi^{2}$ & $0.913 \pm 0.004$ & $0.946 \pm 0.001$ & $0.904 \pm 0.006$ \\
\hline
\end{tabular}

Table 6.7: $N-1$ Plug EM Object Identification Efficiency with and without extra minimum-bias interactions using $Z \rightarrow e e$ events.

Although adding extra minimum-bias interactions to the Monte Carlo helps the plug simulation better match the data, the extra minimum-bias events hurt the simulation's description of the central electron and photon variables. Thus, while there is extra energy in the plug detector that is not simulated properly in the default simulation, its source is not entirely due to extra interactions.

Figure 6.8(c) shows that the plug ID efficiency in data increases with $E_{T}$. So, the kinematics of the physics process being simulated should be considered when determining how to quantify the effect of the extra energy in the plug region. For physics with kinematics similar to $Z \rightarrow e e$ kinematics, such as $Z \gamma, W Z$, and $Z Z$ events, the simulated plug efficiency is scaled by $0.953\left(=\epsilon_{\text {data }} / \epsilon_{\text {sim }}=0.89 / 0.934\right)$. However, higher $E_{T}$ plug electrons and photons, such as those resulting from e* events, will not be as influenced by the extra energy and should not be treated in the same manner. Section 7.2 describes how we deal with this difference.

Figure 6.8 shows the plug EM object identification efficiency as a function of $\eta_{d}, \phi, E_{T}$, and run number. The efficiency is stable with time. The comparisons between data and simulation for the $\eta_{d}, \phi$, and $E_{T}$ are good. The simulation efficiency was scaled at each point by $0.953\left(=\epsilon_{\text {data }} / \epsilon_{\text {sim }}=0.89 / 0.934\right)$ in these 
plots.

\subsubsection{Summary}

We have measured and compared in data and simulation the identification efficiencies of central electrons, central photons, and plug EM objects. The efficiency of central electrons in data is $(94.0 \pm 0.3($ stat $)) \%$ with a systematic error of $1 \%$ due to data-simulation differences.

The "emulated" efficiency of central photons is measured using $Z \rightarrow e e$ electrons to "emulate" the photon cuts. Two separate measurements are performed: one with no $E / p$ cut and one with $0.9<E / p<1.1$ to suppress the effects of bremsstrahlung radiation. The efficiencies in data are $(75.5 \pm 0.7($ stat $)) \%$ and $(84.6 \pm 0.7($ stat $)) \%$ without and with the $E / p$ cut, respectively. There is a $3 \%$ systematic error quoted due to data-simulation differences.

Finally, the plug EM object efficiency is measured using central-plug $Z \rightarrow e e$ data. For electrons with kinematics typical of $Z \rightarrow e e$ events, the simulation over-estimates the plug efficiency. Specifically, the efficiency is measured as $(89.0 \pm$ $0.6($ stat $)) \%$ in data and $(93.4 \pm 0.1($ stat $)) \%$ in simulation. Thus, for simulated plug electrons and photons with kinematics like those of typical $Z \rightarrow e e$ electrons (such as $Z / \gamma^{*}+\gamma, W Z$, and $Z Z$ ), we scale the plug EM object efficiency by $0.953(=$ $\left.\epsilon_{\text {data }} / \epsilon_{\text {sim }}=0.89 / 0.934\right)$. However, the difference between data and simulation decreases as the electron $E_{T}$ increases. In Section 7.3.4, we discuss the treatment of the high $E_{T}$ simulated plug objects in $e^{*}$ events. 


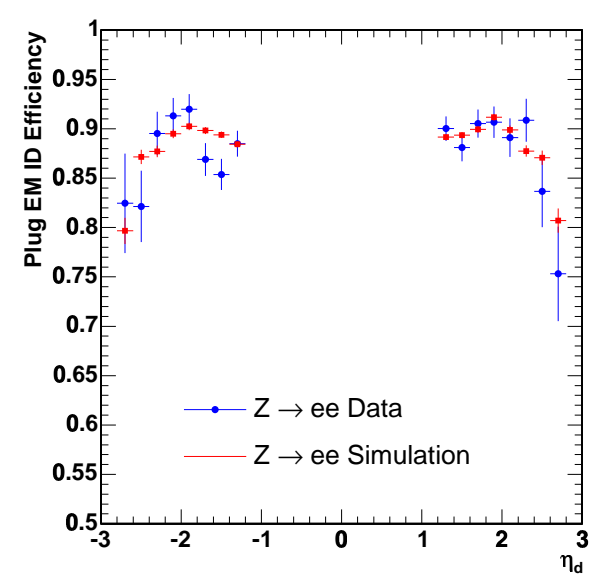

(a)

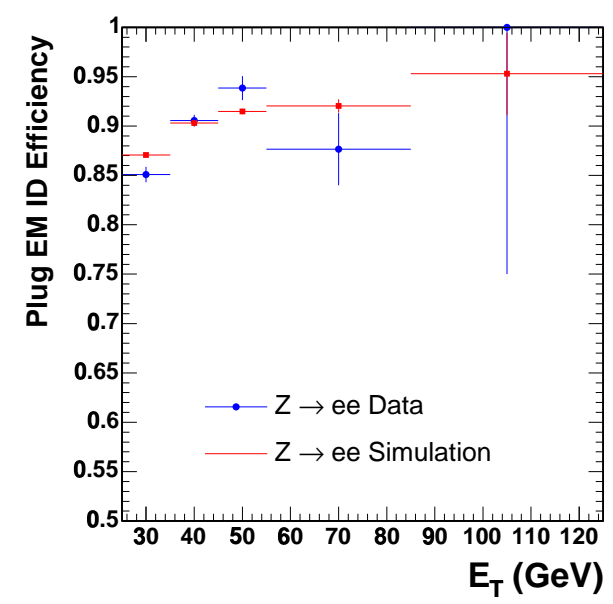

(c)

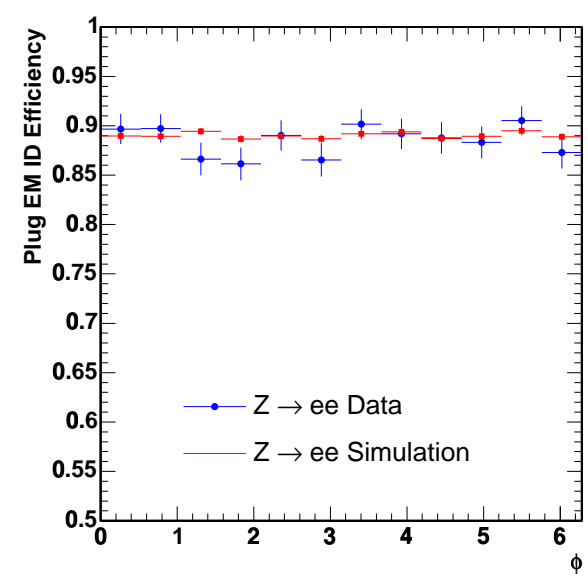

(b)

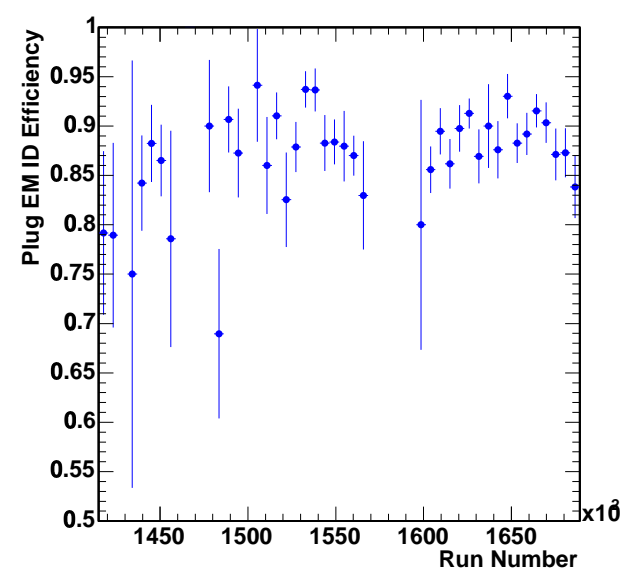

(d)

Figure 6.8: Plug EM object identification efficiency as a function of $\eta_{d}, \phi, E_{T}$, and run number. 


\subsection{Trigger Efficiency}

The signal data are selected from the ELECTRON_CENTRAL_18 and ELECTRON70_L2_JET trigger paths. The requirements of the triggers are defined in Sections 4.1.1 and 4.1.2. The efficiency of the ELECTRON_CENTRAL_18 is described in [28].

There exists a series of "backup" triggers which are designed to measure the efficiency of the signal trigger paths. For example, there is a "W no-track" trigger, W_NOTRACK, which carries all the same calorimeter criteria as the ELECTRON_CENTRAL_18 trigger but no track requirements. So, this trigger can be used to measure the XFT and level-3 tracking efficiency.

This is done by selecting a base sample of events which trigger on the W_NOTRACK trigger and pass a set of tight electron identification criteria and a $\mathbb{E}_{T}$ requirement. The efficiency of the tracking requirements of the trigger is the fraction of base sample events that also fire the ELECTRON_CENTRAL_18 trigger $\left(\epsilon_{\text {trigger }}^{18}\right)$ :

$$
\epsilon_{\text {trigger }}^{18}=\frac{\# \text { of events that also fire ELECTRON_CENTRAL_18 trigger }}{\# \text { of events that fire } \mathrm{W} \_ \text {NOTRACK and pass } \mathrm{W} \rightarrow \mathrm{e} \nu \text { selection }}
$$

The trigger efficiency is measured for several different run periods which are chosen based upon detector performance and changes in trigger requirements. The luminosity weighted average trigger efficiency of the ELECTRON_CENTRAL_18 trigger path is $(96.21 \pm 0.06($ stat $)) \%$ [28].

The ELECTRON_CENTRAL_18 trigger inefficiency is primarily due to the XFT trigger requirements at level-1. For $E_{T} \gtrsim 30 \mathrm{GeV}$, the calorimeter trigger requirements are $100 \%$ efficient [28].

The ELECTRON70_L2_JET trigger has no track requirements and very loose 
calorimeter requirements. This trigger becomes efficient for electrons with energies higher than the trigger threshold. We assume that for electrons that this trigger is $\sim 100 \%$ efficient for $E_{T}>120 \mathrm{GeV}$. This is confirmed by measuring the efficiency of the ELECTRON70_L2_JET trigger with respect to the ELECTRON_CENTRAL_18 trigger $\left(\epsilon_{\text {trigger }}^{70}\right)$. We define a base sample of events that pass the ELECTRON_CENTRAL_18 trigger and have one and only one central electron. The analysis sample consists of events in the base sample that also pass the ELECTRON70_L2_JET trigger. The efficiency is then defined as:

$$
\epsilon_{\text {trigger }}^{70}=\frac{\text { number of events in analysis sample }}{\text { number of events in base sample }}
$$

Figure 6.9 shows the ELECTRON70_L2_JET trigger efficiency with respect to the ELECTRON_CENTRAL_18 trigger as a function of electron $E_{T}$.

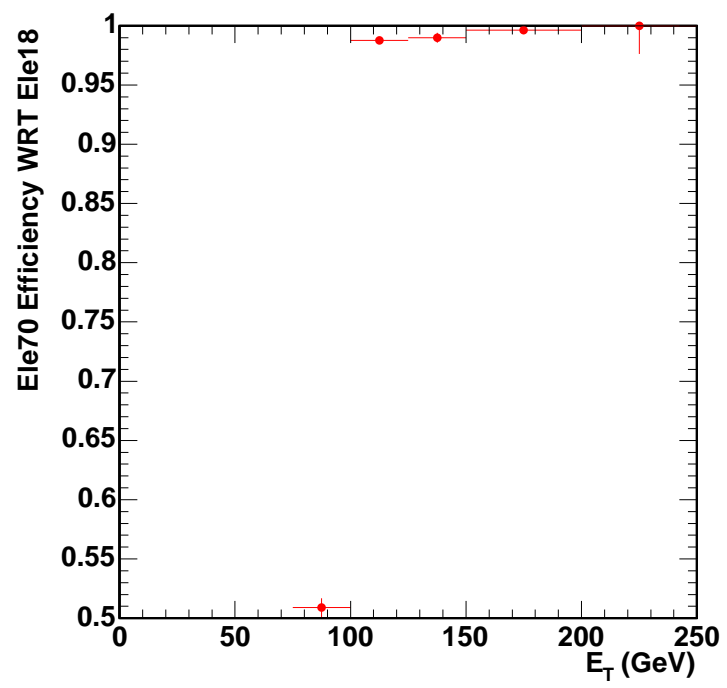

Figure 6.9: ELECTRON70_L2_JET trigger efficiency with respect to the ELECTRON_CENTRAL_18 trigger as a function of electron $E_{T}$. 


\section{3 $Z$ Vertex Efficiency}

The $Z$ vertex of each event, as defined by the $Z$ vertex of the highest $E_{T}$ central electron, is required to be less than $60 \mathrm{~cm}$. The luminosity reported by the Cherenkov Luminosity Counter (CLC) is not restricted to this region, but rather the full luminous region of the $p \bar{p}$ interactions. Thus, the fraction of events produced within 60 $\mathrm{cm}$ must be predicted or measured. The simulation does not reliably model this. Instead the $Z$ vertex efficiency as measured in the data is applied to acceptance calculations. This efficiency is measured to be $\epsilon_{v t x}=0.951 \pm 0.005$ [29].

The measurement of the $Z$ vertex efficiency is described in detail in reference [29]. Minimum-bias data are used to measure the $Z_{v t x}$ distribution and is fit to the following $p \bar{p}$ beam luminosity function [30]:

$$
\frac{d \mathcal{L}(z)}{d z}=N_{\circ} \frac{\exp \left(\frac{-z^{2}}{2 \sigma_{z}^{2}}\right)}{1+\left(\frac{z-z_{\min }}{\beta^{*}}\right)^{2}}
$$

where $z$ is the $Z_{v t x}, N_{\circ}$ is a normalization factor related to the $p, \bar{p}$ fluxes, and $\beta^{*}$ and $\sigma_{z}$ are transverse beam widths defined in [30]. The $\mathrm{Z}$ vertex efficiency for a given range $\left(Z_{c u t}\right)$ can then be measured by:

$$
\epsilon_{Z_{v t x}}=\frac{\int_{-Z_{c u t} \mathrm{~cm}}^{+Z_{\text {cm }}} d \mathcal{L}(z)}{\int_{-\infty \mathrm{cm}}^{+\infty \mathrm{cm}} d \mathcal{L}(z)}
$$

where $Z_{c u t}$ is $60 \mathrm{~cm}$ in this analysis. The efficiency is computed on a run-by-run basis and then luminosity weighted to get the average $Z_{v t x}$ efficiency of $0.951 \pm 0.005$. 


\subsection{Summary}

The central electron, central photon, and plug EM object selection efficiencies were measured in data and simulation using $Z \rightarrow e e$ events. The differences between the data and simulation were studied and used to determine systematic errors. Additionally, the trigger efficiencies and $Z_{v t x}$ efficiency measurements were discussed. 


\section{Chapter 7}

\section{Signal Acceptance}

Having measured the efficiency of identifying central electrons, central photons, and plug EM objects and understood the differences between data and simulation, we can now use simulation to determine the fraction of $e^{*}$ events that would be detected at CDF. This fraction is called "acceptance".

Ideally, the acceptance would be $100 \%$. However, there are several sources of acceptance loss. A first source is the fiducial, or geometric, cuts on electrons and photons that require their EM clusters be located in a well instrumented region of the central or plug detector. The kinematic requirement that fiducial electrons and photons have $E_{T}>25 \mathrm{GeV}$ also causes a loss in acceptance. Additionally, the requirements for electron and photon identification, the trigger, and the $\mathrm{Z}$ vertex lower the fraction of detectable events.

For each acceptance plot, three distributions will be displayed: the central acceptance where both electron candidates and the photon candidate are located in the central region of the detector, the plug acceptance where one electron candidate is central and at least one object is in the plug, and the total acceptance which is simply the addition of the central and plug acceptances. 


\subsection{Maximum Acceptance}

We first discuss the "maximum acceptance" which is the geometric and kinematic acceptance without any object identification, trigger, or $Z_{v t x}$ requirements. Thus, the maximum acceptance is defined as the fraction of generated events which pass fiducial and kinematic cuts. To measure this, we start by requiring one central EM cluster with an associated track. The cluster $E_{T}$ must be greater than 25 $\mathrm{GeV}$, track $p_{T}$ greater than $10 \mathrm{GeV}$, and pass the fiducial requirements described in Chapter 4. Then two more fiducial EM clusters with $E_{T}$ greater than $25 \mathrm{GeV}$ are required. These two clusters have no track requirements. The track vertex of the maximum $E_{T}$ central cluster with a track is used for measuring the transverse energy of the other two clusters. This measurement does not consider the identification efficiencies, trigger efficiency, $Z$ vertex efficiency, or dielectron mass cut efficiency.

\subsubsection{Contact Interaction $e^{*}$}

Figure 7.1 shows the maximum acceptance for the contact interaction $e^{*}$ model for $e^{*}$ masses between $100 \mathrm{GeV}$ and $1000 \mathrm{GeV}$. At low mass, the maximum acceptance is $\sim 32 \%$ and rises to $\sim 49 \%$ at high mass. This is a reflection of the mass dependence of $E_{T}$ and $\eta$, as discussed in Section 2.4.2. Figure 7.1 reproduces the $\eta$ dependence that was shown in Figures 2.10 - Figures 2.12 in that the plug distribution starts decreasing while the central acceptance increases with increasing $e^{*}$ mass.

\subsubsection{Gauge Mediated $e^{*}$}

Figure 7.2(a) shows the maximum acceptance for the gauge mediated $e^{*}$ model for $M_{e^{*}}=100 \mathrm{GeV}$ to $M_{e^{*}}=500 \mathrm{GeV}$. Figure $7.2(\mathrm{~b})$ has the maximum acceptance 


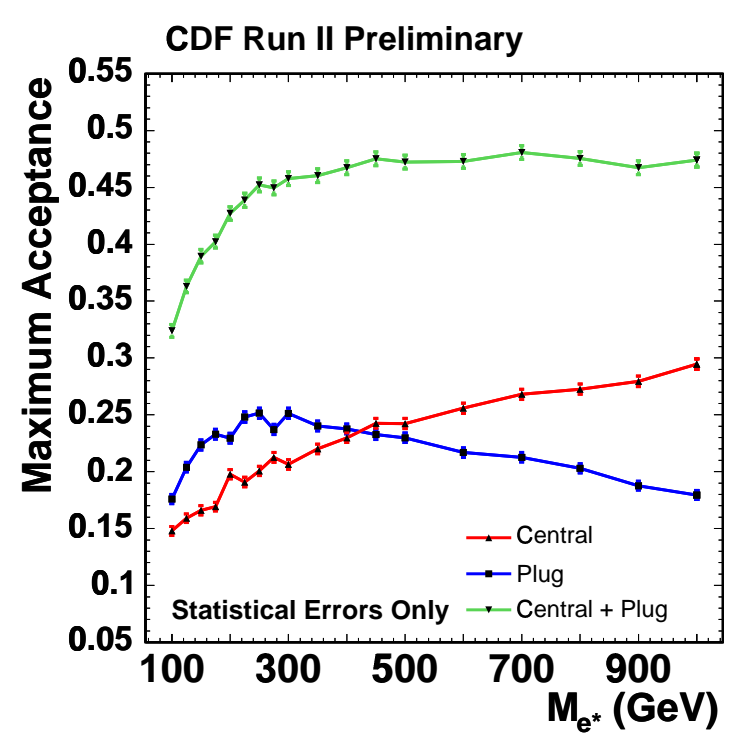

Figure 7.1: Maximum acceptance for contact interaction model.

for the contact interaction $e^{*}$ model for $M_{e^{*}}=100 \mathrm{GeV}$ to $M_{e^{*}}=500 \mathrm{GeV}$, for comparison. The gauge mediated $e^{*}$ model has maximum acceptance of $\sim 28 \%$ for $M_{e^{*}}=100 \mathrm{GeV}$, which is lower than the contact interaction model acceptance. However, the acceptance rises quicker than the contact interaction model acceptance and stabilizes at $\sim 52 \%$.

\subsection{Total Signal Acceptance}

Having measured the maximum acceptance that only took into account the kinematic and fiducial acceptances, we now measure the total signal acceptance which takes into consideration the effect of the electron, photon and plug EM object identification, trigger, $Z_{v t x}$, and dielectron mass requirements. Thus, the total signal acceptance is defined as:

$$
A_{t o t}=\epsilon_{Z_{v t x}} \cdot \epsilon_{t r g} \cdot \epsilon_{M_{e e}} \cdot \epsilon_{i d} \cdot \epsilon_{f i d k i n}
$$




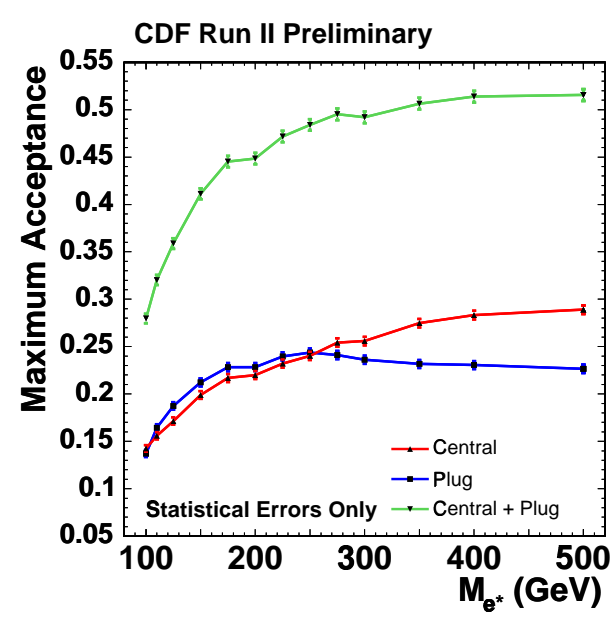

(a)

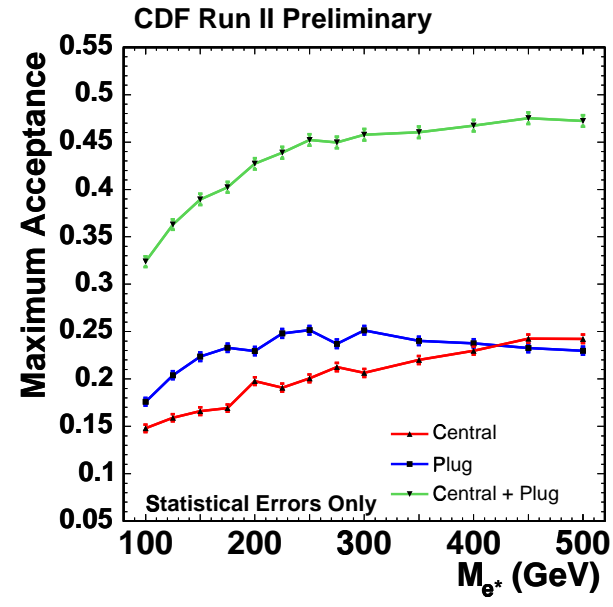

(b)

Figure 7.2: Figure (a) is the maximum acceptance for the gauge mediated $e^{*}$ production. Figure (b) shows the maximum acceptance for contact interaction $e^{*}$ production for $M_{e^{*}}$ up to $500 \mathrm{GeV}$ for comparison with the gauge mediated model.

where $\epsilon_{Z_{v t x}}$ is the efficiency of $\left|Z_{v t x}\right|<60 \mathrm{~cm}, \epsilon_{t r g}$ is the trigger efficiency, $\epsilon_{M_{e e}}$ is the efficiency of the dielectron mass cut which excludes masses in the window of the $Z$ boson resonance, $\epsilon_{i d}$ is the electron, photon, and plug EM object identification efficiency, and $\epsilon_{f i d k i n}$ is the fiducial and kinematic acceptance of the $e^{*}$ model.

\subsubsection{Contact Interaction $e^{*}$}

The total signal acceptance for contact interaction $e^{*}$ production is shown in Figure 7.3. For $e^{*}$ mass equal to $100 \mathrm{GeV}$, the total signal acceptance is particularly low due to the $M_{e e}$ cut. This is especially true for the plug acceptance because if an event contains two plug objects and their two-body invariant mass is in the $Z$ boson mass range, the event is vetoed. As the $e^{*}$ mass increases away from the $Z$ pole, this effect no longer contributes significantly to the acceptance. For masses greater than $\sim 275 \mathrm{GeV}$, the total signal acceptance is $\sim 31 \%$. 


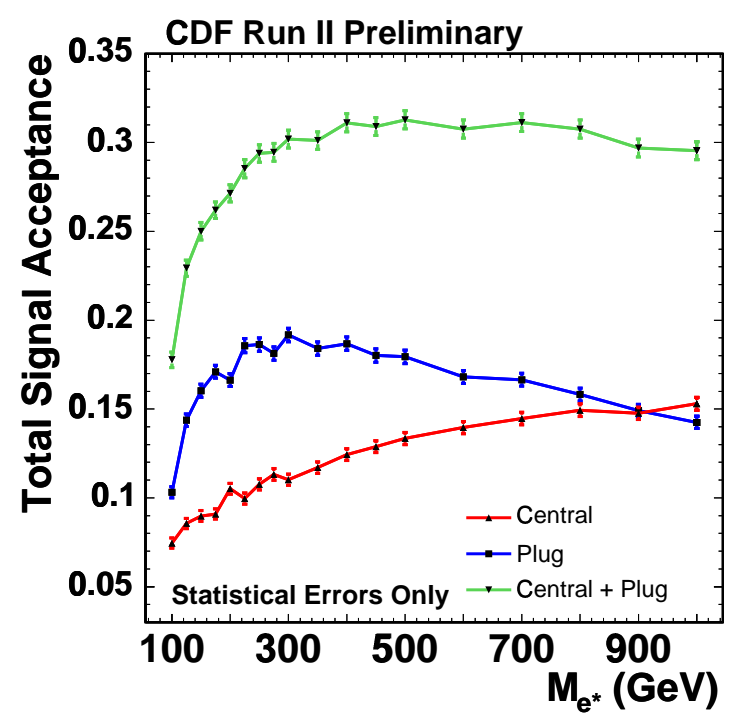

Figure 7.3: Total signal acceptance for contact interaction model.

\subsubsection{Gauge Mediated $e^{*}$}

The total signal acceptance for gauge mediated $e^{*}$ production is shown in Figure 7.4(a) for masses between $100 \mathrm{GeV}$ and $500 \mathrm{GeV}$. For easy comparison, Figure $7.4(\mathrm{~b})$ is the contact interaction model acceptance in the same mass range. With the exception of the effect of the dielectron mass cut, which only affects low masses, the shape of the total acceptance distributions are primarily determined by the geometrical and kinematic properties of the $e^{*}$ models. The major difference between the total acceptance and maximum acceptance is that the total acceptance at each mass point is suppressed due to inefficiencies in the object selection criteria, trigger requirements, $Z_{v t x}$ cut, and $M_{e e}$ cut. 


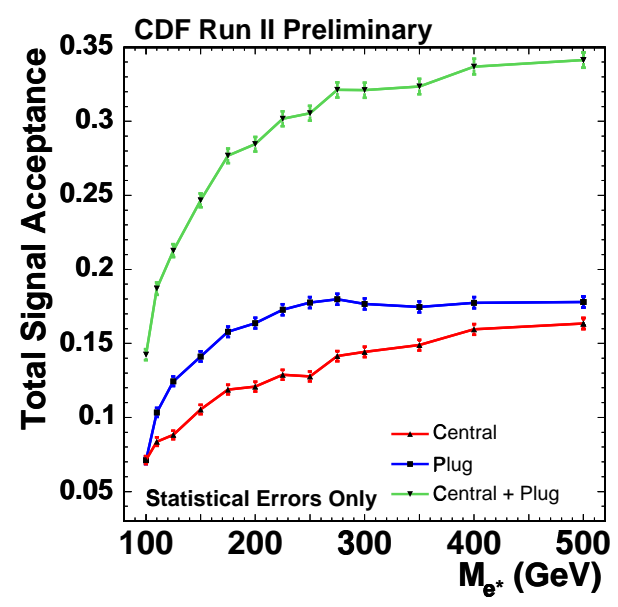

(a)

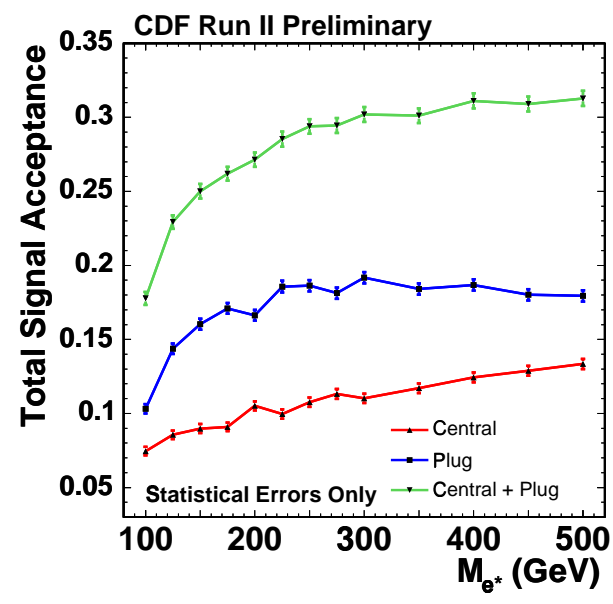

(b)

Figure 7.4: Figure (a) is the total signal acceptance for the gauge mediated $e^{*}$ production. Figure (b) shows the total signal acceptance for contact interaction $e^{*}$ production for $M_{e^{*}}$ up to $500 \mathrm{GeV}$ for comparison with the gauge mediated model.

\subsection{Contact Interaction Model Acceptance Un- certainties}

The uncertainties addressed in this section are from studies of the simulated contact interaction Monte Carlo samples.

\subsubsection{Statistical Error}

For each mass value, 9500 to $10000 e^{*}$ events are generated and simulated. Binomial errors are used to determine the uncertainty due to the statistics of the samples ${ }^{1}$. The statistical error at each mass point is shown in Table 7.1. The statistical percent error averaged over all masses is $1.75 \%$.

${ }^{1}$ The binomial error is given by $\sqrt{\epsilon(1-\epsilon) / N}$, where $\mathrm{N}$ is the number of events. 


\begin{tabular}{|l|c|}
\hline Mass $(\mathrm{GeV})$ & $\begin{array}{c}\text { Statistical } \\
\text { Error } \Delta A / A(\%)\end{array}$ \\
\hline \hline 100 & 2.39 \\
\hline 125 & 1.96 \\
\hline 150 & 1.96 \\
\hline 175 & 1.80 \\
\hline 200 & 1.77 \\
\hline 225 & 1.80 \\
\hline 250 & 1.68 \\
\hline 275 & 1.70 \\
\hline 300 & 1.65 \\
\hline 350 & 1.66 \\
\hline 400 & 1.63 \\
\hline 450 & 1.64 \\
\hline 500 & 1.63 \\
\hline 600 & 1.65 \\
\hline 700 & 1.63 \\
\hline 800 & 1.65 \\
\hline 900 & 1.69 \\
\hline 1000 & 1.69 \\
\hline \hline Average & 1.75 \\
\hline & \\
\hline
\end{tabular}

Table 7.1: Relative statistical error on total signal acceptance for the contact interaction model. 


\subsubsection{Parton Distribution Functions}

Parton Distribution Functions were described in Section 1.1.4. Event kinematics depend on the choice of parton distribution functions (PDFs). This dependence is included as a systematic error on the acceptance. The PDFs used for the central value are CTEQ5L. The effect of different PDFs on the acceptance is investigated by generating 5000 events for each $e^{*}$ mass at the HEPG (parton) level using the CTEQ3L, CTEQ3M, CTEQ5M1, GRV94M, and GRV94L parton distribution functions. A summary of the systematic error due to each PDF set is shown in Table 7.2.

The acceptance at HEPG level is compared to the CTEQ5L HEPG acceptance. The $\%$ differences shown in Table 7.2 are defined as:

$$
\% \operatorname{Diff}_{\mathrm{PDF}}=\frac{\mathrm{A}_{\mathrm{PDF}}^{\mathrm{HEPG}}-\mathrm{A}_{\mathrm{CTEQ}}^{\mathrm{HEPG}}}{\mathrm{A}_{\mathrm{CTEQ}}^{\mathrm{HEPG}}} * 100
$$

The far right column is the maximum PDF \% difference for each mass value. Because the PDF uncertainty is measured with independent samples, the statistical error on $\triangle A^{H E P G} / A^{H E P G}$ can be easily calculated. The statistical errors on the acceptances are propagated to determine the statistical uncertainty on the PDF \% difference. The statistical uncertainty on $\triangle A^{H E P G} / A^{H E P G}$ is calculated to be $\sim$ 1\%. With the statistics available, these differences can not be deemed significant, so the average PDF relative uncertainty, $1.04 \%$, is used for all mass points.

\subsubsection{Extra Minimum-Bias Events}

In Section 6.1.4, the plug EM object identification efficiency is discussed. For $Z \rightarrow e e$ electrons, the simulation overestimates the efficiency and requires a correction factor of $0.953\left(=\epsilon_{\text {data }} / \epsilon_{\text {sim }}=0.89 / 0.934\right)$. This difference is attributed to extra energy in 


\begin{tabular}{|l|c|c|c|c|c|c|}
\hline Mass & $\begin{array}{c}\text { CTEQ3L } \\
\text { \% Diff }\end{array}$ & $\begin{array}{c}\text { CTEQ3M } \\
\text { \% Diff }\end{array}$ & $\begin{array}{c}\text { CTEQ5M1 } \\
\text { \% Diff }\end{array}$ & $\begin{array}{c}\text { GRV94L } \\
\text { \% Diff }\end{array}$ & $\begin{array}{c}\text { GRV94M } \\
\text { \% Diff }\end{array}$ & $\begin{array}{c}\text { Maximum } \\
\text { \% Diff }\end{array}$ \\
\hline \hline 100 & -0.51 & -1.44 & -0.98 & -1.24 & -0.17 & $1.44 \pm 1.03$ \\
\hline 125 & 1.55 & 1.86 & 2.77 & 1.72 & 1.77 & $2.77 \pm 0.98$ \\
\hline 150 & -0.40 & 0.15 & 0.31 & 0.07 & 0.06 & $0.40 \pm 0.88$ \\
\hline 175 & -0.73 & -0.29 & 0.36 & 0.04 & 0.65 & $0.73 \pm 0.82$ \\
\hline 200 & -1.80 & -0.54 & 1.33 & -0.46 & 0.84 & $1.80 \pm 0.78$ \\
\hline 225 & -0.90 & 0.18 & 0.05 & -0.15 & 0.46 & $0.90 \pm 0.75$ \\
\hline 250 & -0.27 & -0.39 & 0.34 & -0.22 & -0.91 & $0.91 \pm 0.73$ \\
\hline 275 & 0.47 & 0.31 & -0.25 & -0.14 & 0.15 & $0.47 \pm 0.72$ \\
\hline 300 & 0.24 & 0.36 & 0.90 & 0.39 & -0.55 & $0.90 \pm 0.71$ \\
\hline 350 & -0.38 & 0.48 & 0.28 & -0.07 & -0.31 & $0.48 \pm 0.68$ \\
\hline 400 & -0.63 & 0.76 & 0.49 & -0.38 & 0.06 & $0.76 \pm 0.67$ \\
\hline 450 & 0.71 & 1.30 & 0.54 & 1.50 & 0.31 & $1.50 \pm 0.67$ \\
\hline 500 & 0.05 & -0.35 & 0.59 & -0.11 & -0.06 & $0.59 \pm 0.66$ \\
\hline 600 & -0.47 & -0.54 & -0.57 & -0.32 & -1.06 & $1.06 \pm 0.66$ \\
\hline 700 & 0.31 & -0.53 & -0.37 & 0.09 & 0.38 & $0.53 \pm 0.67$ \\
\hline 800 & 0.38 & 0.17 & -1.26 & -0.48 & 0.04 & $1.26 \pm 0.69$ \\
\hline 900 & 0.80 & -0.06 & -0.05 & 0.13 & -1.32 & $1.32 \pm 0.72$ \\
\hline 1000 & 0.96 & 0.51 & -0.57 & 0.42 & -0.66 & $0.96 \pm 0.74$ \\
\hline \hline Average & & & & & & 1.04 \\
\hline
\end{tabular}

Table 7.2: Relative difference between various PDF acceptances and the CTEQ5L acceptance. 
the plug region that is not modeled properly in the simulation and primarily affected the PEM $\chi^{2}$ (see Table 6.7). The efficiency of plug EM objects was reproduced by adding extra minimum-bias interactions to the simulation, essentially throwing extra energy to the plug region. However, these extra minimum-bias interactions hurt the central region cuts, so we study the effect of extra minimum-bias interaction on plug EM objects in the $e^{*}$ simulation.

Table 7.3 shows the plug ID efficiency at three $e^{*}$ mass points with and without extra interactions. The average ratio of plug ID efficiency with extra minimumbias interactions to the default Monte Carlo efficiency is 0.993. Therefore, in the analysis, we use the default Monte Carlo and weigh the plug object efficiency by 0.993. We estimate the systematic error on this weight factor to be 0.0035 , half the difference between the weight factor and 1.

\begin{tabular}{|l|c|c|c|}
\hline$e^{*}$ Mass & $\begin{array}{c}\text { Efficiency Without } \\
\text { Extra Interactions }\end{array}$ & $\begin{array}{c}\text { Efficiency With } \\
\text { Extra Interactions }\end{array}$ & Scale Factor \\
\hline \hline 100 & $0.964 \pm 0.003($ stat $)$ & $0.953 \pm 0.004($ stat $)$ & 0.989 \\
\hline 500 & $0.974 \pm 0.003($ stat $)$ & $0.965 \pm 0.003($ stat $)$ & 0.991 \\
\hline 900 & $0.977 \pm 0.002($ stat $)$ & $0.975 \pm 0.003($ stat $)$ & 0.999 \\
\hline
\end{tabular}

Table 7.3: Plug EM object identification efficiency in simulated $e^{*}$ Monte Carlo with and without extra interactions. Also shown is the scale factor: $\frac{\text { Efficiency With Extra Interactions }}{\text { Efficiency Without Extra Interactions }}$.

The decision to use the 0.993 scale factor for plug objects in the simulation is based upon the assumption that the plug EM object efficiency increases with $E_{T}$. Figure 7.5 shows the distribution of efficiency as a function of $E_{T}$. Within errors, the data shows a rise in $\epsilon_{p}$ with $E_{T}$, confirming the prediction from the simulation. 


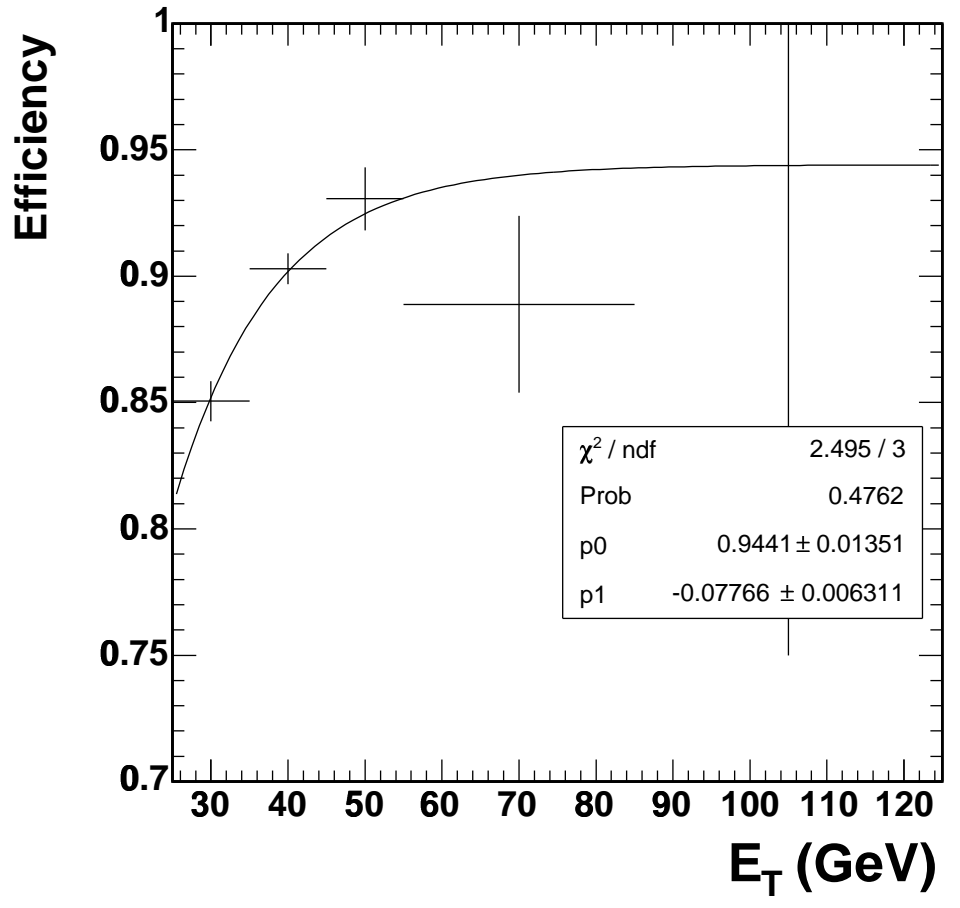

Figure 7.5: Plug EM object ID efficiency $\left(\epsilon_{p}\right)$ as a function of $E_{T}$. It is fit to $\epsilon=p_{0} \cdot\left(1-e^{p_{1} \cdot E_{T}}\right)$. 


\subsubsection{Identification Efficiency}

The systematic error on the central electron identification efficiency is 1\%. Each central electron in an event is weighted by 1 and then the weight is fluctuated by $0.01(1 \%)$ to obtain the error on total signal acceptance due to uncertainty in the central electron identification efficiency measurement.

The systematic error quoted on the central photon identification efficiency is $3 \%$. Similarly, each central photon is given a weight of 1 and then the effect of fluctuating this weight by $0.03(3 \%)$ is studied. From this we obtain the error due to photon ID efficiency.

Plug objects are weighted by $0.993 \pm 0.0035$. The error on the acceptance due to the error on the weight factor is determined by fluctuating the central value of the weight by its error.

Errors due to ID efficiencies are shown in Table 7.4. The average error due to uncertainty in object identification efficiency is $2.63 \%$. Statistical errors on the relative error are not quoted because it is measured using the same sample and changing the weights of objects in the sample.

\subsubsection{Trigger Efficiency}

As discussed in Section 6.2, the efficiency of the L3_ELECTRON_CENTRAL_18 trigger is measured to be $(96.21 \pm 0.06) \%$. Reference [28] discusses in detail the trigger efficiency. The calorimeter requirements of the L3_ELECTRON_CENTRAL_18 trigger are fully efficient for $E_{T} \gtrsim 30 \mathrm{GeV}$. The track requirements, however, are not, and it is these requirements that reduce the L3_ELECTRON_CENTRAL_18 trigger efficiency.

Events with two central electrons have two chances to fire the trigger. The trigger 


\begin{tabular}{|l|c|c|c|c|}
\hline Mass $(\mathrm{GeV})$ & $\begin{array}{c}\text { Central } \\
\text { Electron } \\
\text { ID } \epsilon_{e} \\
\Delta A / A(\%)\end{array}$ & $\begin{array}{c}\text { Central } \\
\text { Photon } \\
\text { ID } \epsilon_{\gamma}\end{array}$ & $\begin{array}{c}\text { Plug } \\
\text { EM Object } \\
\text { ID } \epsilon_{p} \\
\Delta / A(\%)\end{array}$ & $\begin{array}{c}\text { Total } \\
\text { ID } \epsilon \\
\Delta A / A(\%)\end{array}$ \\
\hline \hline 100 & 1.59 & 2.13 & 0.25 & 2.67 \\
\hline 125 & 1.56 & 1.98 & 0.28 & 2.53 \\
\hline 150 & 1.56 & 1.96 & 0.28 & 2.53 \\
\hline 175 & 1.57 & 1.92 & 0.28 & 2.50 \\
\hline 200 & 1.60 & 2.05 & 0.26 & 2.61 \\
\hline 225 & 1.59 & 1.94 & 0.27 & 2.52 \\
\hline 250 & 1.59 & 2.01 & 0.26 & 2.57 \\
\hline 275 & 1.60 & 2.04 & 0.26 & 2.61 \\
\hline 300 & 1.59 & 2.02 & 0.26 & 2.59 \\
\hline 350 & 1.61 & 2.06 & 0.25 & 2.62 \\
\hline 400 & 1.61 & 2.10 & 0.24 & 2.66 \\
\hline 450 & 1.62 & 2.10 & 0.24 & 2.66 \\
\hline 500 & 1.62 & 2.12 & 0.24 & 2.68 \\
\hline 600 & 1.63 & 2.15 & 0.23 & 2.71 \\
\hline 700 & 1.61 & 2.20 & 0.23 & 2.74 \\
\hline 800 & 1.63 & 2.17 & 0.23 & 2.73 \\
\hline 900 & 1.62 & 2.19 & 0.23 & 2.74 \\
\hline 1000 & 1.64 & 2.19 & 0.22 & 2.75 \\
\hline \hline Average & 1.60 & 2.07 & 0.25 & 2.63 \\
\hline
\end{tabular}

Table 7.4: Relative systematic errors due to identification efficiencies on the contact interaction model total signal acceptance. 
efficiency for events with two central electrons is given by $\epsilon_{t r g}^{e e}=\epsilon_{t r g}^{e}+\left(1-\epsilon_{t r g}^{e}\right) \epsilon_{t r g}^{e}=$ $99.9 \%$. Thus, there is no need to scale for trigger efficiency if the event has two central electrons.

The ELECTRON70_L2_JET has no Level 1 or Level 2 track requirements (see Section 4.1.2). At Level 3, a track with $p_{T}>15 \mathrm{GeV}$ is required. According to [28], the Level 3 tracking efficiency is $99.5 \%$. As discussed in Section 6.2, we conclude that for $E_{T}>120 \mathrm{GeV}$, the ELECTRON70_L2_JET becomes fully efficient. Thus, we apply the trigger efficiency to events with only one central electron with $E_{T}<120$ $\mathrm{GeV}$.

Two errors due to trigger efficiency are calculated. One is due to the error on the L3_ELECTRON_CENTRAL_18 trigger, $(96.21 \pm 0.06) \%$. The second is due to the possibility that the ELECTRON70_L2_JET trigger is not fully efficiency for $E_{T}>120 \mathrm{GeV}$. To account for this, the difference in acceptance between using a cut of $E_{T}>120 \mathrm{GeV}$ and $E_{T}>150 \mathrm{GeV}$ is quoted. The two sources of error are assumed to be uncorrelated.

Errors due to trigger efficiency are shown in Table 7.5. The dependence of $\Delta A / A$ due to trigger efficiency error is a reflection of the kinematic behavior of $e^{*}$ production as a function of mass. Thus, an relative error of $0.3 \%$, the maximum $\Delta A / A$ which is reported at $M_{e^{*}}=300 \mathrm{GeV}$, is used. The statistical error on $\Delta A / A$ due to the trigger efficiency uncertainty is not reported since $\Delta A / A$ is not measured from an independent sample.

\subsection{6 $Z_{v t x}$ Efficiency}

Section 6.3 described the measurement of the fraction of events whose interaction vertex falls within $60 \mathrm{~cm}$ in $Z$ of the center of the detector. This $Z$ vertex efficiency 


\begin{tabular}{|l|c|c|c|}
\hline Mass (GeV) & $\begin{array}{c}\text { ELECTRON_18 } \\
\text { Trigger Error } \\
\Delta A / A(\%)\end{array}$ & $\begin{array}{c}\text { ELECTRON_70 } \\
\text { Trigger Error } \\
\Delta A / A(\%)\end{array}$ & $\begin{array}{c}\text { Total } \\
\text { Trigger Error } \\
\Delta A / A(\%)\end{array}$ \\
\hline \hline 100 & 0.017 & 0.169 & 0.170 \\
\hline 125 & 0.018 & 0.193 & 0.193 \\
\hline 150 & 0.016 & 0.219 & 0.220 \\
\hline 175 & 0.015 & 0.245 & 0.246 \\
\hline 200 & 0.013 & 0.246 & 0.246 \\
\hline 225 & 0.012 & 0.265 & 0.265 \\
\hline 250 & 0.011 & 0.281 & 0.281 \\
\hline 275 & 0.010 & 0.287 & 0.287 \\
\hline 300 & 0.007 & 0.301 & 0.301 \\
\hline 350 & 0.005 & 0.256 & 0.256 \\
\hline 400 & 0.004 & 0.210 & 0.210 \\
\hline 450 & 0.003 & 0.145 & 0.145 \\
\hline 500 & 0.003 & 0.119 & 0.119 \\
\hline 600 & 0.003 & 0.113 & 0.113 \\
\hline 700 & 0.003 & 0.099 & 0.099 \\
\hline 800 & 0.003 & 0.093 & 0.093 \\
\hline 900 & 0.004 & 0.084 & 0.084 \\
\hline 1000 & 0.004 & 0.103 & 0.103 \\
\hline \hline Average & 0.008 & 0.190 & 0.191 \\
\hline
\end{tabular}

Table 7.5: Systematics on the contact interaction model total signal acceptance due to trigger efficiency uncertainty. Errors are relative. 
is measured to be $\epsilon_{v t x}=0.951 \pm 0.005$. The error on the $Z$ vertex efficiency measurement translates to a $0.53 \%$ relative error on the total signal acceptance at all mass points.

\subsubsection{Extra Material in the Simulation}

Comparisons of data and the 4.9.1 default simulation showed that the material in the detector was not properly modeled and that it was necessary to "add" extra "phantom" layers of passive material to the default simulation to better model the detector. Studies of the $E / p$ and $Q \cdot \Delta X$ distributions for electrons in the central tracking region showed that adding a cylinder of $(4.5 \pm 1.5) \% X_{\circ}{ }^{2}$ of material was necessary in the central region. Because copper cables were not sufficiently accounted for in the material description in GEANT, a cylinder of copper is "added" to the simulation in the central region [27]. In the plug region, comparisons of the plug pre-radiator (PPR) energy response from electrons were used to determine that $(1 / 3 \pm 1 / 6) X_{\circ}$ of material in front of the plug calorimeter needed to be added. Due to the absence of the silicon readout cables in the plug region, iron is used as the extra material added to the default simulation in the plug region [27].

The effect of adding the phantom layers of extra material on the total signal acceptance is determined by studying the central and plug acceptances separately. The change in central acceptance due to adding the extra materials is divided by 3 to obtain the error because the error on the amount of material in the central region is $1 / 3$ of the amount of material added. Similarly, the effect of extra material on the

\footnotetext{
${ }^{2} X_{\circ}$ is a unit of radiation length, where a radiation length is defined as the average distance over which electrons with high energy lose all but $1 / e$ if their energy via bremsstrahlung radiation. $7 / 9 X_{\circ}$ is the conversion length which is defined as the distance over which a high energy photon loses energy via pair production [2].
} 
plug acceptance is divided by 2 to obtain the error in the plug region. The central and plug errors are assumed to be correlated and are added linearly to obtain the total error.

For simplicity, the acceptances calculated in Tables 7.6 - 7.8 are calculated without the trigger efficiency, $Z$ vertex efficiency, and energy scaling and smearing applied. This does not affect the relative error measurement.

Table 7.8 shows the average uncertainty on the total signal acceptance (central+plug) is $1.44 \%$. The errors on the relative error that are shown in the last column of Tables 7.6 - 7.8 are the propagated statistical errors. These show that the fluctuations as a function of mass are not statistically significant. However, it is logical that the acceptance for low mass $e^{*}$ events, such as $M_{e^{*}}=100 \mathrm{GeV}$, is more affected by the material. So, for $M_{e^{*}}=100 \mathrm{GeV}$, we choose to use $\Delta A / A=3 \%$ due to missing material in the simulation. For the remaining mass points $\left(M_{e^{*}}>100\right.$ $\mathrm{GeV})$, the average $\Delta A / A$, which is $1.44 \%$, is used.

\subsubsection{Energy Scale}

The energy scale is discussed in Chapter 5. We determined a 0.001 energy scale error in the central calorimeters and 0.002 energy scale uncertainty in the plug calorimeters. The energies of central (plug) objects are multiplied by $1 \pm 0.001$ $(1 \pm 0.002)$ to determine the uncertainty due to energy scale.

Systematics due to energy scale are shown in Table 7.9. The west and east plug scale factor errors are added in quadrature because they are not correlated. The central scale factor error is correlated with the west and east plug energy and so they are added linearly.

As in the case of the extra material uncertainty and the PDF uncertainty, fluc- 


\begin{tabular}{|l|c|c|c|c|}
\hline Mass (GeV) & $\begin{array}{c}\text { Acceptance } \\
\text { With Extra } \\
\text { Material }\end{array}$ & $\begin{array}{c}\text { Acceptance } \\
\text { Without Extra } \\
\text { Material }\end{array}$ & $\begin{array}{c}\text { Error Due } \\
\text { to Extra } \\
\text { Material=(diff/3) }\end{array}$ & $\begin{array}{c}\text { Relative } \\
\text { Error } \\
\Delta A / A(\%)\end{array}$ \\
\hline \hline 100 & 0.0786 & 0.0888 & 0.0034 & $4.33 \pm 1.87$ \\
\hline 125 & 0.0900 & 0.0947 & 0.0016 & $1.74 \pm 1.55$ \\
\hline 150 & 0.0944 & 0.0998 & 0.0018 & $1.90 \pm 1.56$ \\
\hline 175 & 0.0956 & 0.0997 & 0.0014 & $1.42 \pm 1.49$ \\
\hline 200 & 0.1113 & 0.1163 & 0.0017 & $1.50 \pm 1.36$ \\
\hline 225 & 0.1048 & 0.1112 & 0.0021 & $2.03 \pm 1.51$ \\
\hline 250 & 0.1127 & 0.1219 & 0.0031 & $2.71 \pm 1.39$ \\
\hline 275 & 0.1189 & 0.1283 & 0.0031 & $2.62 \pm 1.35$ \\
\hline 300 & 0.1160 & 0.1221 & 0.0021 & $1.77 \pm 1.95$ \\
\hline 350 & 0.1231 & 0.1335 & 0.0035 & $2.83 \pm 1.32$ \\
\hline 400 & 0.1304 & 0.1420 & 0.0039 & $2.99 \pm 1.31$ \\
\hline 450 & 0.1355 & 0.1478 & 0.0041 & $3.03 \pm 1.24$ \\
\hline 500 & 0.1404 & 0.1492 & 0.0029 & $2.08 \pm 1.19$ \\
\hline 600 & 0.1470 & 0.1609 & 0.0046 & $3.15 \pm 1.18$ \\
\hline 700 & 0.1524 & 0.1614 & 0.0030 & $1.96 \pm 1.13$ \\
\hline 800 & 0.1573 & 0.1708 & 0.0045 & $2.85 \pm 1.12$ \\
\hline 900 & 0.1553 & 0.1682 & 0.0043 & $2.76 \pm 1.13$ \\
\hline 1000 & 0.1611 & 0.1790 & 0.0060 & $3.72 \pm 1.12$ \\
\hline \hline Average & & & & 2.52 \\
\hline
\end{tabular}

Table 7.6: Systematics on the contact interaction model central acceptance due to extra material. 


\begin{tabular}{|l|c|c|c|c|}
\hline Mass $(\mathrm{GeV})$ & $\begin{array}{c}\text { Acceptance } \\
\text { With Extra } \\
\text { Material }\end{array}$ & $\begin{array}{c}\text { Acceptance } \\
\text { Without Extra } \\
\text { Material }\end{array}$ & $\begin{array}{c}\text { Error Due } \\
\text { to Extra } \\
\text { Material=(diff/2) }\end{array}$ & $\begin{array}{c}\text { Relative } \\
\text { Error } \\
\Delta A / A(\%)\end{array}$ \\
\hline \hline 100 & 0.1139 & 0.1186 & 0.0024 & $2.08 \pm 2.12$ \\
\hline 125 & 0.1553 & 0.1588 & 0.0017 & $1.11 \pm 1.63$ \\
\hline 150 & 0.1726 & 0.1803 & 0.0038 & $2.23 \pm 1.59$ \\
\hline 175 & 0.1846 & 0.1872 & 0.0013 & $0.71 \pm 1.45$ \\
\hline 200 & 0.1789 & 0.1859 & 0.0035 & $1.95 \pm 1.50$ \\
\hline 225 & 0.1988 & 0.1966 & -0.0011 & $-0.55 \pm 1.42$ \\
\hline 250 & 0.1999 & 0.2064 & 0.0032 & $1.62 \pm 1.38$ \\
\hline 275 & 0.1935 & 0.1944 & 0.0004 & $0.22 \pm 1.40$ \\
\hline 300 & 0.2052 & 0.2063 & 0.0006 & $0.27 \pm 1.96$ \\
\hline 350 & 0.1961 & 0.1974 & 0.0006 & $0.32 \pm 1.38$ \\
\hline 400 & 0.1987 & 0.2063 & -0.0021 & $-1.04 \pm 1.38$ \\
\hline 450 & 0.1919 & 0.1974 & 0.0001 & $0.05 \pm 1.39$ \\
\hline 500 & 0.1911 & 0.1946 & 0.0011 & $0.60 \pm 1.41$ \\
\hline 600 & 0.1791 & 0.1921 & 0.0022 & $1.22 \pm 1.49$ \\
\hline 700 & 0.1776 & 0.1934 & 0.0010 & $0.56 \pm 1.48$ \\
\hline 800 & 0.1686 & 0.1835 & 0.0013 & $0.77 \pm 1.54$ \\
\hline 900 & 0.1592 & 0.1795 & 0.00005 & $0.03 \pm 1.58$ \\
\hline 1000 & 0.1519 & 0.1712 & -0.0006 & $-0.41 \pm 1.62$ \\
\hline \hline Average & & & & 0.65 \\
\hline
\end{tabular}

Table 7.7: Systematics on the contact interaction model plug acceptance due to extra material. 


\begin{tabular}{|l|c|c|c|}
\hline Mass $(\mathrm{GeV})$ & $\begin{array}{c}\text { Acceptance } \\
\text { With Extra } \\
\text { Material }\end{array}$ & $\begin{array}{c}\text { Error Due } \\
\text { to Extra } \\
\text { Material }\end{array}$ & $\begin{array}{c}\text { Relative } \\
\text { Error } \\
\Delta A / A(\%)\end{array}$ \\
\hline \hline 100 & 0.1925 & 0.0058 & $3.00 \pm 1.51$ \\
\hline 125 & 0.2453 & 0.0033 & $1.34 \pm 1.19$ \\
\hline 150 & 0.2670 & 0.0056 & $2.11 \pm 1.18$ \\
\hline 175 & 0.2802 & 0.0027 & $0.95 \pm 1.09$ \\
\hline 200 & 0.2902 & 0.0052 & $1.78 \pm 1.07$ \\
\hline 225 & 0.3036 & 0.0010 & $0.34 \pm 1.08$ \\
\hline 250 & 0.3126 & 0.0063 & $2.01 \pm 1.03$ \\
\hline 275 & 0.3124 & 0.0035 & $1.14 \pm 1.03$ \\
\hline 300 & 0.3212 & 0.0026 & $0.81 \pm 1.44$ \\
\hline 350 & 0.3192 & 0.0041 & $1.29 \pm 1.01$ \\
\hline 400 & 0.3291 & 0.0018 & $0.56 \pm 1.00$ \\
\hline 450 & 0.3273 & 0.0042 & $1.29 \pm 0.99$ \\
\hline 500 & 0.3315 & 0.0041 & $1.23 \pm 0.97$ \\
\hline 600 & 0.3261 & 0.0068 & $2.09 \pm 1.00$ \\
\hline 700 & 0.3300 & 0.0040 & $1.21 \pm 0.97$ \\
\hline 800 & 0.3259 & 0.0060 & $1.78 \pm 0.99$ \\
\hline 900 & 0.3145 & 0.0043 & $1.38 \pm 1.00$ \\
\hline 1000 & 0.3129 & 0.0054 & $1.71 \pm 1.01$ \\
\hline \hline Average & & & 1.44 \\
\hline
\end{tabular}

Table 7.8: Systematics on the contact interaction model total (central+plug) acceptance due to extra material. 
tuations in the error due to energy scale are not statistically significant. However, due to the low energy tail of the energy distribution, the uncertainty due to energy scale for $e^{*}$ mass equal to $100 \mathrm{GeV}$ may be greater than at higher mass values. Thus, for $M_{e^{*}}=100 \mathrm{GeV}$, the $\Delta A / A$ value of $0.46 \%$ is used. The average, $0.13 \%$, is used for all masses greater than $100 \mathrm{GeV}$.

\begin{tabular}{|l|c|c|c|c|}
\hline Mass $(\mathrm{GeV})$ & $\begin{array}{c}\text { Central Scale } \\
\Delta A / A(\%)\end{array}$ & $\begin{array}{c}\text { West Plug Scale } \\
\Delta A / A(\%)\end{array}$ & $\begin{array}{c}\text { East Plug Scale } \\
\Delta A / A(\%)\end{array}$ & $\begin{array}{c}\text { Total Scale } \\
\Delta A / A(\%)\end{array}$ \\
\hline \hline 100 & 0.27 & 0.06 & 0.18 & 0.46 \\
\hline 125 & 0.15 & 0.05 & 0.13 & 0.29 \\
\hline 150 & 0.03 & 0.06 & 0.06 & 0.12 \\
\hline 175 & 0.04 & 0.17 & 0.12 & 0.25 \\
\hline 200 & 0.03 & 0.06 & 0.05 & 0.11 \\
\hline 225 & 0.06 & 0.04 & 0.02 & 0.11 \\
\hline 250 & 0.02 & 0.04 & 0.05 & 0.10 \\
\hline 275 & 0.09 & 0.09 & 0.02 & 0.19 \\
\hline 300 & 0.04 & 0.03 & 0.02 & 0.08 \\
\hline 350 & 0.02 & 0.07 & 0.05 & 0.10 \\
\hline 400 & 0.02 & 0.02 & 0.02 & 0.05 \\
\hline 450 & 0.03 & 0.03 & 0. & 0.06 \\
\hline 500 & 0.01 & 0.01 & 0. & 0.02 \\
\hline 600 & 0.001 & 0.03 & 0.05 & 0.06 \\
\hline 700 & 0.02 & 0.04 & 0.07 & 0.10 \\
\hline 800 & 0.02 & 0.04 & 0.01 & 0.07 \\
\hline 900 & 0.01 & 0.04 & 0.03 & 0.07 \\
\hline 1000 & 0.01 & 0.07 & 0.07 & 0.11 \\
\hline \hline Average & 0.05 & 0.05 & 0.05 & 0.13 \\
\hline
\end{tabular}

Table 7.9: Systematics on the contact interaction model total acceptance due to energy scaling.

\subsubsection{Energy Resolution}

In Chapter 5 we compared the energy resolution of the data to the resolution in the simulation. It was necessary to smear central electrons and photons by $(3.17 \pm$ 
$0.23) \%$, west plug EM objects by $(1.90 \pm 0.65) \%$, and east plug EM objects by $(1.90 \pm 0.80) \%$ in the simulation. This section shows the effect of the smearing uncertainty on the acceptance measurement.

The systematic uncertainties due to energy resolution are shown in Table 7.10. The fluctuations in uncertainty due to energy resolution are statistical, except at $M_{e^{*}}=100 \mathrm{GeV}$, where it is reasonable that energy resolution would have a greater effect on the acceptance. Thus, we use $0.29 \%$ for $\Delta A / A$ at $M_{e^{*}}=100 \mathrm{GeV}$, and the average, $0.13 \%$, for $M_{e^{*}}>100 \mathrm{GeV}$.

\begin{tabular}{|c|c|c|c|c|}
\hline Mass $(\mathrm{GeV})$ & $\begin{array}{c}\text { Central } \\
\text { Smearing } \\
\Delta A / A(\%)\end{array}$ & $\begin{array}{c}\text { West Plug } \\
\text { Smearing } \\
\Delta A / A(\%)\end{array}$ & $\begin{array}{c}\text { East Plug } \\
\text { Smearing } \\
\Delta A / A(\%)\end{array}$ & $\begin{array}{c}\text { Total Energy } \\
\text { Smearing } \\
\Delta A / A(\%)\end{array}$ \\
\hline 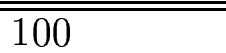 & 年.06 & 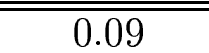 & $\overline{0.19}$ & 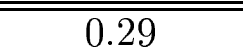 \\
\hline 125 & 0.11 & 0.17 & 0.04 & 0.29 \\
\hline 150 & 0.03 & 0.07 & 0.13 & 0.19 \\
\hline 175 & 0.04 & 0.10 & 0.12 & 0.20 \\
\hline 200 & 0.04 & 0.05 & 0.05 & 0.11 \\
\hline 225 & 0.08 & 0.04 & 0.04 & 0.14 \\
\hline 250 & 0.01 & 0.10 & 0.08 & 0.13 \\
\hline 275 & 0.09 & 0.08 & 0.11 & 0.23 \\
\hline 300 & 0.03 & 0.03 & 0.04 & 0.09 \\
\hline 350 & 0.02 & 0.04 & 0.08 & 0.11 \\
\hline 400 & 0.02 & 0.03 & 0.05 & 0.08 \\
\hline 450 & 0.04 & 0.01 & 0.01 & 0.06 \\
\hline 500 & 0.03 & 0.04 & 0.04 & 0.10 \\
\hline 600 & 0.01 & 0.06 & 0.05 & 0.10 \\
\hline 700 & 0.03 & 0.05 & 0.03 & 0.10 \\
\hline 800 & 0.03 & 0.02 & 0.04 & 0.08 \\
\hline 900 & 0.01 & 0.02 & 0.03 & 0.05 \\
\hline 1000 & 0.01 & 0.02 & 0.03 & 0.05 \\
\hline$\overline{~ A v e r a g e ~}$ & $\overline{0.04}$ & 0.06 & 0.07 & 0.13 \\
\hline
\end{tabular}

Table 7.10: Systematics on the contact interaction model total signal acceptance due to energy smearing corrections. 


\subsubsection{Summary}

Table 7.11 has a summary of the contact interaction model total signal acceptance and uncertainty contributions due to each error source at a series of mass values. All sources of error are assumed uncorrelated. The total error at each mass point is determined by adding the individual errors in quadrature. For $M_{e^{*}}=100 \mathrm{GeV}$, we use $5.0 \%$ for $\Delta A / A$. For $M_{e^{*}}>100 \mathrm{GeV}$, we use $3.7 \%$. This reflects the average $\Delta A / A$ due to each source of error, with the exception of the trigger efficiency, where the maximum $\Delta A / A$ due to trigger efficiency is used.

\begin{tabular}{|l|c|c|c|c|c|c|c|c|c|}
\hline Mass & Acc & Stat & PDF & Trig & $Z_{v t x}$ & ID & Energy & Mat & Total \\
\hline \hline 100 & 0.178 & 2.39 & 1.44 & 0.17 & 0.53 & 2.67 & 0.55 & 3.00 & 4.96 \\
\hline 125 & 0.229 & 1.96 & 2.76 & 0.19 & 0.53 & 2.53 & 0.41 & 1.34 & 4.50 \\
\hline 150 & 0.250 & 1.96 & 0.40 & 0.22 & 0.53 & 2.53 & 0.22 & 2.11 & 3.90 \\
\hline 175 & 0.262 & 1.80 & 0.73 & 0.25 & 0.53 & 2.50 & 0.32 & 0.95 & 3.37 \\
\hline 200 & 0.271 & 1.77 & 1.80 & 0.25 & 0.53 & 2.61 & 0.15 & 1.78 & 4.09 \\
\hline 225 & 0.285 & 1.79 & 0.90 & 0.27 & 0.53 & 2.52 & 0.18 & 0.34 & 3.30 \\
\hline 250 & 0.294 & 1.68 & 0.91 & 0.28 & 0.53 & 2.57 & 0.06 & 2.01 & 3.83 \\
\hline 275 & 0.294 & 1.70 & 0.47 & 0.29 & 0.53 & 2.61 & 0.30 & 1.14 & 3.42 \\
\hline 300 & 0.302 & 1.65 & 0.90 & 0.30 & 0.53 & 2.59 & 0.12 & 0.81 & 3.36 \\
\hline 350 & 0.301 & 1.66 & 0.48 & 0.26 & 0.53 & 2.62 & 0.15 & 1.29 & 3.45 \\
\hline 400 & 0.311 & 1.63 & 0.76 & 0.21 & 0.53 & 2.66 & 0.10 & 0.56 & 3.31 \\
\hline 450 & 0.309 & 1.64 & 1.50 & 0.15 & 0.53 & 2.66 & 0.09 & 1.29 & 3.74 \\
\hline 500 & 0.313 & 1.63 & 0.58 & 0.12 & 0.53 & 2.68 & 0.10 & 1.23 & 3.46 \\
\hline 600 & 0.308 & 1.65 & 1.06 & 0.11 & 0.53 & 2.71 & 0.11 & 2.09 & 3.98 \\
\hline 700 & 0.311 & 1.63 & 0.53 & 0.10 & 0.53 & 2.74 & 0.14 & 1.21 & 3.50 \\
\hline 800 & 0.308 & 1.65 & 1.26 & 0.09 & 0.53 & 2.73 & 0.11 & 1.78 & 3.90 \\
\hline 900 & 0.297 & 1.69 & 1.32 & 0.08 & 0.53 & 2.74 & 0.09 & 1.38 & 3.78 \\
\hline 1000 & 0.295 & 1.69 & 0.96 & 0.10 & 0.53 & 2.75 & 0.12 & 1.71 & 3.82 \\
\hline \hline$M_{e^{*}>100 \mathrm{GeV}}$ & & 1.75 & 1.04 & 0.30 & 0.53 & 2.63 & 0.15 & 1.44 & 3.69 \\
\hline
\end{tabular}

Table 7.11: Total signal acceptance and a summary of the uncertainties for the contact interaction model. All errors are $\Delta A / A$ in percentage. The bottom row shows the value used for $M_{e^{*}}>=125 \mathrm{GeV}$. For $M_{e^{*}}=100 \mathrm{GeV}$, we use the value shown in the top row. 


\subsection{Gauge Mediated Model Acceptance Uncer- tainties}

In Section 7.3 the uncertainty measurements for the contact interaction model acceptance due to the following sources were described: statistical, PDFs, identification efficiency, trigger efficiency, $Z_{v t x}$ efficiency, extra material in the simulation, energy scale, and energy resolution. To a good approximation, the error due to PDFs, extra material, and $Z_{v t x}$ efficiency will be the same for the gauge mediated model acceptances. We remeasure the uncertainties due to statistics, ID efficiencies, trigger efficiency, energy scale, and energy resolution.

\subsubsection{Statistical Uncertainty}

We generate 9000-10,000 events at each mass value. The statistical uncertainties are shown in Table 7.1. The average relative uncertainty is $1.82 \%$.

\subsubsection{Identification Efficiency}

The method for measuring the uncertainty due to identification efficiency was described in Section 7.3.4. We apply the same method to the gauge mediated model acceptances. The uncertainties are shown in Table 7.13.

\subsubsection{Trigger Efficiency}

The measurement of the uncertainty due to trigger efficiency is described in Section 7.3.5. Errors due to uncertainty in the trigger efficiency are shown in Table 7.14. The dependence of $\Delta A / A$ due to trigger efficiency error is a reflection of the kinematic behavior of $e^{*}$ production as a function of mass. We conservatively use a 


\begin{tabular}{|l|c|}
\hline Mass $(\mathrm{GeV})$ & $\begin{array}{c}\text { Statistical } \\
\text { Error } \Delta A / A(\%)\end{array}$ \\
\hline \hline 100 & 2.61 \\
\hline 110 & 2.15 \\
\hline 125 & 2.17 \\
\hline 150 & 1.89 \\
\hline 175 & 1.86 \\
\hline 200 & 1.73 \\
\hline 225 & 1.66 \\
\hline 250 & 1.73 \\
\hline 275 & 1.60 \\
\hline 300 & 1.60 \\
\hline 350 & 1.59 \\
\hline 400 & 1.55 \\
\hline 500 & 1.54 \\
\hline \hline Average & 1.82 \\
\hline
\end{tabular}

Table 7.12: Relative statistical error on total signal acceptance for the gauge mediated model.

relative error of $0.3 \%$ for all mass points, the maximum $\Delta A / A$, which is reported at $M_{e^{*}}=300 \mathrm{GeV}$.

\subsubsection{Energy Scale}

The method for determining the acceptance uncertainty due to the energy scale is described in Section 7.3.8. Table 7.15 shows the uncertainties due to energy scale at each mass point. We use $0.31 \%$ for $\Delta A / A$ at $M_{e^{*}}=100 \mathrm{GeV}$ and $110 \mathrm{GeV}$. The average uncertainty, $0.18 \%$, is used for all masses greater than $100 \mathrm{GeV}$.

\subsubsection{Energy Resolution}

The method for determining the acceptance uncertainty due to the energy resolution is described in Section 7.3.9. Table 7.15 shows the uncertainties due to energy 


\begin{tabular}{|c|c|c|c|c|}
\hline Mass $(\mathrm{GeV})$ & $\begin{array}{c}\text { Central } \\
\text { Electron } \\
\text { ID } \epsilon \\
\Delta A / A(\%)\end{array}$ & $\begin{array}{c}\text { Central } \\
\text { Photon } \\
\text { ID } \epsilon \\
\Delta A / A(\%)\end{array}$ & $\begin{array}{c}\text { Plug } \\
\text { EM Object } \\
\text { ID } \epsilon \\
\Delta A / A(\%)\end{array}$ & $\begin{array}{c}\text { Total } \\
\text { ID } \epsilon \\
\Delta A / A(\%)\end{array}$ \\
\hline$\overline{100}$ & $\overline{\overline{1.69}}$ & 2.18 & $\overline{0.21}$ & 2.77 \\
\hline$\overline{110}$ & 1.64 & 2.06 & 0.24 & 2.65 \\
\hline 125 & 1.64 & 2.00 & 0.25 & 2.59 \\
\hline 150 & 1.67 & 2.00 & 0.24 & 2.62 \\
\hline 175 & 1.67 & 2.00 & 0.24 & 2.62 \\
\hline 200 & 1.68 & 1.99 & 0.23 & 2.62 \\
\hline 225 & 1.68 & 2.01 & 0.23 & 2.63 \\
\hline 250 & 1.69 & 1.97 & 0.23 & 2.61 \\
\hline 275 & 1.69 & 2.03 & 0.22 & 2.65 \\
\hline 300 & 1.69 & 2.07 & 0.22 & 2.68 \\
\hline 350 & 1.70 & 2.04 & 0.22 & 2.67 \\
\hline 400 & 1.70 & 2.07 & 0.22 & 2.69 \\
\hline 500 & 1.69 & 2.05 & 0.22 & 2.67 \\
\hline Average & 1.68 & 2.04 & 0.23 & 2.65 \\
\hline
\end{tabular}

Table 7.13: Relative systematic errors due to identification efficiencies for the gauge mediated model total signal acceptance. 


\begin{tabular}{|l|c|c|c|}
\hline Mass $(\mathrm{GeV})$ & $\begin{array}{c}\text { ELECTRON_18 } \\
\text { Trigger Error } \\
\Delta A / A(\%)\end{array}$ & $\begin{array}{c}\text { ELECTRON_70 } \\
\text { Trigger Error } \\
\Delta A / A(\%)\end{array}$ & $\begin{array}{c}\text { Total } \\
\text { Trigger Error } \\
\Delta A / A(\%)\end{array}$ \\
\hline \hline 100 & 0.017 & 0.078 & 0.080 \\
\hline 110 & 0.019 & 0.109 & 0.111 \\
\hline 125 & 0.019 & 0.128 & 0.129 \\
\hline 150 & 0.015 & 0.171 & 0.172 \\
\hline 175 & 0.014 & 0.208 & 0.208 \\
\hline 200 & 0.012 & 0.244 & 0.244 \\
\hline 225 & 0.010 & 0.267 & 0.267 \\
\hline 250 & 0.009 & 0.278 & 0.278 \\
\hline 275 & 0.007 & 0.278 & 0.279 \\
\hline 300 & 0.006 & 0.291 & 0.291 \\
\hline 350 & 0.005 & 0.234 & 0.234 \\
\hline 400 & 0.004 & 0.171 & 0.171 \\
\hline 500 & 0.003 & 0.125 & 0.125 \\
\hline \hline Average & 0.011 & 0.199 & 0.191 \\
\hline
\end{tabular}

Table 7.14: Systematic uncertainties on the total signal acceptance due to trigger efficiency uncertainties for the Gauge Mediated model. 


\begin{tabular}{|l|c|c|c|c|}
\hline Mass $(\mathrm{GeV})$ & $\begin{array}{c}\text { Central Scale } \\
\Delta A / A(\%)\end{array}$ & $\begin{array}{c}\text { West Plug Scale } \\
\Delta A / A(\%)\end{array}$ & $\begin{array}{c}\text { East Plug Scale } \\
\Delta A / A(\%)\end{array}$ & $\begin{array}{c}\text { Total Scale } \\
\Delta / A(\%)\end{array}$ \\
\hline \hline 100 & 0.18 & 0.07 & 0.09 & 0.31 \\
\hline 110 & 0.14 & 0.10 & 0.08 & 0.28 \\
\hline 125 & 0.12 & 0.02 & 0.13 & 0.25 \\
\hline 150 & 0.12 & 0.03 & 0.08 & 0.21 \\
\hline 175 & 0.13 & 0.05 & 0.07 & 0.22 \\
\hline 200 & 0.11 & 0.05 & 0.14 & 0.26 \\
\hline 225 & 0.01 & 0.01 & 0.05 & 0.07 \\
\hline 250 & 0.02 & 0.08 & 0.04 & 0.12 \\
\hline 275 & 0.14 & 0.07 & 0.05 & 0.22 \\
\hline 300 & 0.01 & 0.05 & 0.02 & 0.07 \\
\hline 350 & 0.05 & 0.02 & 0.06 & 0.12 \\
\hline 400 & 0.04 & 0.05 & 0.11 & 0.16 \\
\hline 500 & 0.02 & 0.04 & 0.04 & 0.07 \\
\hline \hline Average & 0.08 & 0.05 & 0.07 & 0.18 \\
\hline
\end{tabular}

Table 7.15: Systematics on gauge mediated model total acceptance due to energy scaling.

resolution for each mass value. We use $0.50 \%$ for $\Delta A / A$ at $e^{*}$ masses equal to 100 Gev and $110 \mathrm{GeV}$. The average uncertainty, $0.22 \%$, is used for all masses greater than $100 \mathrm{GeV}$.

\subsubsection{Summary}

Table 7.17 summarizes the gauge mediated model total signal acceptance and the uncertainty contributions due to each source at a series of mass values. All sources of error are assumed uncorrelated, and the total error at each mass point is determined by adding the individual errors in quadrature. For $M_{e^{*}}=100 \mathrm{GeV}$ and $110 \mathrm{GeV}$, a relative uncertainty of $5.1 \%$ is used. For $M_{e^{*}}>110 \mathrm{GeV}, 3.8 \%$ is used for $\Delta A / A$. This comes from the average $\Delta A / A$ due to each source of error, with the exception of the trigger efficiency, where the maximum $\Delta A / A$ due to trigger efficiency is used. 


\begin{tabular}{|c|c|c|c|c|}
\hline Mass $(\mathrm{GeV})$ & $\begin{array}{c}\text { Central } \\
\text { Smearing } \\
\Delta A / A(\%)\end{array}$ & $\begin{array}{l}\text { West Plug } \\
\text { Smearing } \\
\Delta A / A(\%)\end{array}$ & $\begin{array}{c}\text { East Plug } \\
\text { Smearing } \\
\Delta A / A(\%)\end{array}$ & $\begin{array}{c}\text { Total Energy } \\
\text { Smearing } \\
\Delta A / A(\%)\end{array}$ \\
\hline 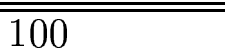 & "0.29 & $\overline{\overline{0.07}}$ & $\overline{\overline{0.11}}$ & $\overline{\overline{0.42}}$ \\
\hline 110 & 0.24 & 0.12 & 0.23 & 0.50 \\
\hline 125 & 0.05 & 0.21 & 0.15 & 0.33 \\
\hline 150 & 0.07 & 0.09 & 0.13 & 0.24 \\
\hline 175 & 0.08 & 0.10 & 0.10 & 0.22 \\
\hline 200 & 0.08 & 0.06 & 0.10 & 0.19 \\
\hline 225 & 0.05 & 0.08 & 0.02 & 0.14 \\
\hline 250 & 0.01 & 0.09 & 0.09 & 0.15 \\
\hline 275 & 0.08 & 0.01 & 0.06 & 0.14 \\
\hline 300 & 0.04 & 0.01 & 0.06 & 0.10 \\
\hline 350 & 0.04 & 0.02 & 0.08 & 0.13 \\
\hline 400 & 0.05 & 0.06 & 0.11 & 0.19 \\
\hline 500 & 0.02 & 0.03 & 0.05 & 0.07 \\
\hline Average & $\overline{0.09}$ & 0.07 & $\overline{0.10}$ & 0.22 \\
\hline
\end{tabular}

Table 7.16: Systematics on gauge mediated total signal acceptance due to energy smearing corrections. 


\begin{tabular}{|l|c|c|c|c|c|c|c|c|c|}
\hline Mass & Acc & Stat & PDF & Trig & $Z_{v t x}$ & ID & Energy & Mat & Total \\
\hline \hline 100 & 0.142 & 2.61 & 1.44 & 0.08 & 0.53 & 2.76 & 0.52 & 3.00 & 5.11 \\
\hline 110 & 0.187 & 2.15 & - & 0.11 & 0.53 & 2.65 & 0.57 & - & - \\
\hline 125 & 0.213 & 2.17 & 2.76 & 0.13 & 0.53 & 2.59 & 0.42 & 1.34 & 4.62 \\
\hline 150 & 0.247 & 1.89 & 0.40 & 0.17 & 0.53 & 2.62 & 0.32 & 2.11 & 3.93 \\
\hline 175 & 0.277 & 1.86 & 0.73 & 0.21 & 0.53 & 2.62 & 0.31 & 0.95 & 3.49 \\
\hline 200 & 0.285 & 1.73 & 1.80 & 0.24 & 0.53 & 2.62 & 0.32 & 1.78 & 4.09 \\
\hline 225 & 0.302 & 1.66 & 0.90 & 0.27 & 0.53 & 2.63 & 0.16 & 0.34 & 3.32 \\
\hline 250 & 0.305 & 1.73 & 0.91 & 0.28 & 0.53 & 2.60 & 0.19 & 2.01 & 3.88 \\
\hline 275 & 0.321 & 1.60 & 0.47 & 0.28 & 0.53 & 2.65 & 0.27 & 1.14 & 3.40 \\
\hline 300 & 0.321 & 1.60 & 0.90 & 0.29 & 0.53 & 2.68 & 0.12 & 0.81 & 3.41 \\
\hline 350 & 0.324 & 1.59 & 0.48 & 0.23 & 0.53 & 2.67 & 0.17 & 1.29 & 3.45 \\
\hline 400 & 0.337 & 1.55 & 0.76 & 0.17 & 0.53 & 2.69 & 0.25 & 0.56 & 3.30 \\
\hline 500 & 0.341 & 1.54 & 0.58 & 0.13 & 0.53 & 2.67 & 0.10 & 1.23 & 3.41 \\
\hline \hline$M_{e^{*}}>110 \mathrm{GeV}$ & & 1.82 & 1.04 & 0.29 & 0.53 & 2.65 & 0.29 & 1.44 & 3.79 \\
\hline
\end{tabular}

Table 7.17: Total signal acceptance and uncertainties for the gauge mediated model. All errors are $\Delta A / A$ in percentage. The bottom row shows the value used for $M_{e^{*}}>=110 \mathrm{GeV}$. For $M_{e^{*}}=100$ and $110 \mathrm{GeV}$, the value shown in the top row is used. 


\subsection{Simulation of Electron + Photon Invariant} Mass

Figure 2.5 in Section 2.4.1 demonstrates that for $M_{e^{*}}<\Lambda$ the $e+\gamma$ invariant mass distributions are very narrow. In Figure 7.6 the reconstructed $M_{e \gamma}$ distribution is plotted for three $M_{e^{*}}$ and $\Lambda$ choices. If one compares Figure 7.6 to Figure 2.5, it is clear that for $M_{e^{*}}<\Lambda$ the observed width of the resonance is dominated by detector resolution.

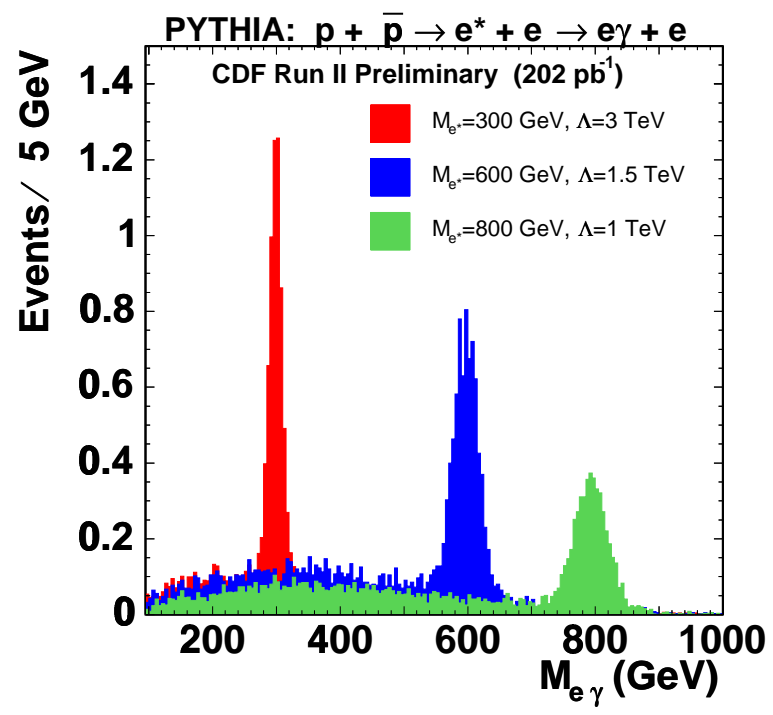

Figure 7.6: Fully simulated electron-photon invariant mass spectra for three choices of $M_{e^{*}}$ and $\Lambda$. 


\section{Chapter 8}

\section{Fake Rates}

A detector background is one in which an object that is not an electron or photon passes the electron or photon selection requirements. For example, a hadronic jet that is formed from a final state quark or gluon could contain hadrons that decay to electrons (e.g. from $b$ decay) or photons (e.g. from $\pi^{\circ}, \eta$ decay). Furthermore, electrons are produced by photon conversions. If an electron or photon decay particle contains a large fraction of the jet energy, it may be misidentified as a single, prompt electron or photon.

To measure background contamination due to QCD jets faking electrons and photons, measurements of "fake rates" are made. The fake rates are defined as the rate at which QCD jets fake a given particle. We start by assuming universal fake rates such that the rate for a given jet does not depend on the properties of other objects in the event. In practice, the process producing the final state particles could be different; an example is differences in quark and gluon fragmentation. We ignore these differences at first order and later study them as sources of systematic uncertainty.

The search signal consists of two electrons, at least one of which has fired the electron trigger. Therefore two fake rates are measured for central electrons: one for a loose central EM object which fires the trigger to fake an electron and a second 
fake rate for an unbiased central jet to fake an electron. Additionally, the signal consists of a central photon for which we measure the unbiased central jet $\rightarrow$ photon fake rate. For plug data, we measure the unbiased jet $\rightarrow$ plug EM object fake rate.

The fake rates are assumed to be independent and can thus be multiplied together to determine the background due to multiple misidentified electrons and photons. Using the four measured fake rates, multijet background can be predicted by re-weighting multijet events from the electron trigger sample. The fake rates are also used to measure $(Z \rightarrow e e)+$ jet, $(W \rightarrow e \nu)+$ jet, and diphoton + jet backgrounds. The re-weighting details are discussed in Chapter 9.

The central jet $\rightarrow$ electron fake rate, central jet $\rightarrow$ photon fake rate, and jet $\rightarrow$ plug EM object fake rates are measured using the QCD enriched samples from the JET_20, JET_50, JET_70, and JET_100 datasets that were described in Section 4.1.3. The trigger EM object $\rightarrow$ electron fake rate is measured using the signal dataset.

\subsection{Unbiased Central Jet $\rightarrow$ Electron Fake Rate}

The central jet $\rightarrow$ electron fake rate is defined as the rate at which central jets are misidentified as electrons. This rate is applied to unbiased jets in the electron signal dataset, or, in other words, those jets that likely did not cause the electron trigger to fire.

\subsubsection{Data Samples}

QCD data triggered on the JET_20, JET_50, JET_70, and JET_100 triggers are used to obtain a sample of unbiased jets. We assume that the maximum $E_{T}$ jet 
in the event is the one that fired the jet trigger. To avoid any bias due to the jet trigger, we do not use the maximum $E_{T}$ jet and thus use only non-leading jets.

\subsubsection{Measurement}

The rate for an unbiased central jet to fake an electron $\left(r_{e}\right)$ is measured by selecting non-leading jets and counting the rate at which electrons are matched to the selected jets. Jets are considered if they are central, have $E_{T}>25 \mathrm{GeV}$, and $\left|Z_{v t x}\right|<$ $60 \mathrm{~cm}$. Electron candidates are selected using the electron selection criteria listed in Table 4.2. A jet is matched to an electron if $\Delta R<0.15$, where $\Delta R=\sqrt{\Delta \eta^{2}+\Delta \phi^{2}}$. This choice is motivated by Figure 8.1, which shows the difference in $\eta-\phi$ space between jets and electrons in an event.

Therefore, the unbiased central jet $\rightarrow$ electron fake rate is defined as $r_{e}=N_{e}^{o} / N_{j}$, where $N_{j}$ is the number of non-leading central jets with $E_{T}>25 \mathrm{GeV}$ and $\left|Z_{v t x}\right|<$ $60 \mathrm{~cm}$, and $N_{e}^{o}$ is the number of observed electrons matched to these jets. The fake rate is dependent on the jet $E_{T}$ and so is measured as a function of $E_{T}$. The unbiased jet to electron fake rate is shown in Figure 8.2.

We study the $\eta_{d}$ dependence of the fake rate. Figure 8.3 shows the measured fake rate as a function of $\eta_{d}$. Excluding the points at $\eta_{d} \sim \pm 1.05$, where the fake rate is low due to fiducial cuts imposed because of detector inefficiency, the weighted average is $0.00044 \pm 0.00001$. The standard deviation of the fake rate as a function of $\eta_{d}$ is $\sim 0.0003$, or $63.3 \%$ relative to the weighted average. The spread in fake rate is incorporated into the systematic error.

The fake rate also depends on whether the jet is the second leading $E_{T}$ jet or one of the remaining lower $E_{T}$ jets in the event. We refer to the second leading $E_{T}$ jet as "Jet2" and all remaining jets with lower $E_{T}$ as "Jet345". Figure 8.4 shows 


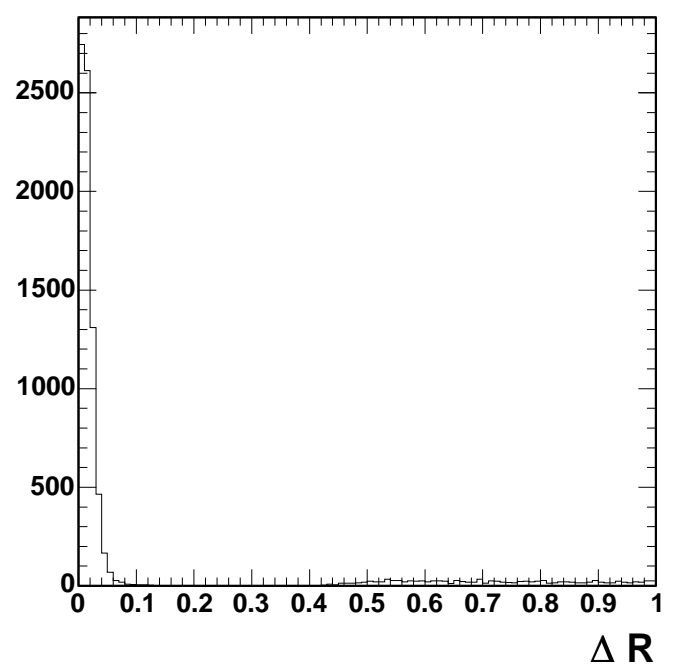

Figure 8.1: $\Delta R$ between jets and electron candidates. We use a cut of $\Delta R<0.15$ to match jets to electrons.

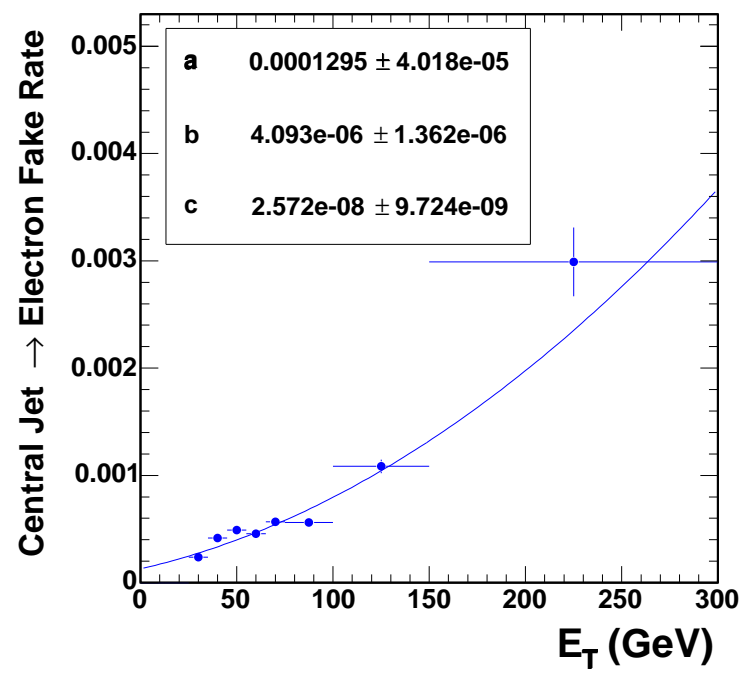

Figure 8.2: Unbiased jet $\rightarrow$ electron fake rate as a function of $E_{T}$. The errors shown are statistical only. Shown are the quadratic fit results where $r_{e}=a+b \cdot E_{T}+c \cdot E_{T}^{2}$. 


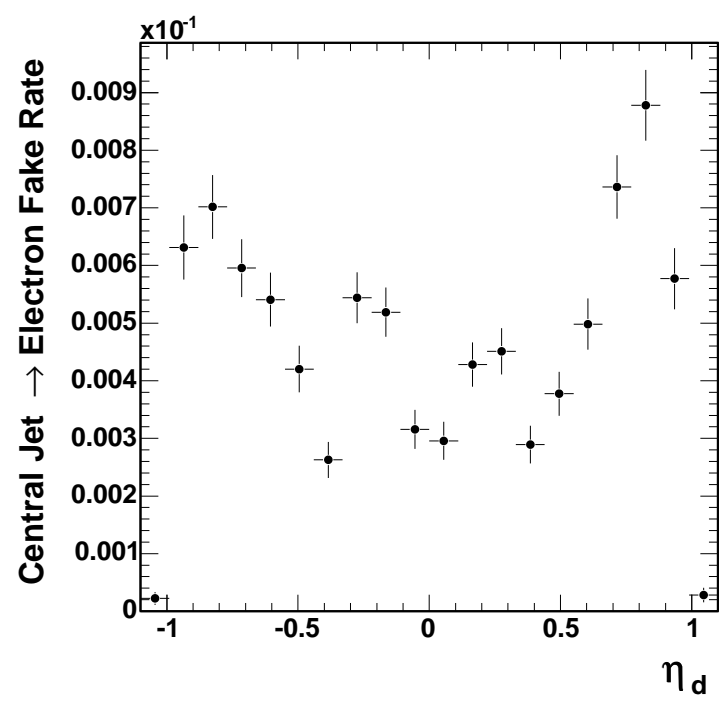

Figure 8.3: Unbiased jet $\rightarrow$ electron fake rate as a function of detector $\eta$. The errors shown are statistical only.

the fake rate distributions for Jet2 and Jet345. Additionally, the combined fake rate for all jets is superposed. For transverse energies less than $55 \mathrm{GeV}$, the fake rates for all jet types are approximately consistent. For transverse energies greater than $55 \mathrm{GeV}$, the fake rate for Jet345 is significantly higher than the combined fake rate. The Jet2 fake rate is not significantly different than the combined fake rate because most jets are Jet2.

In Figure 8.2, the combined fake rate is fit to a quadratic; the fit values are used to obtain the central value of the fake rate at a given transverse energy. Likewise, we apply a fit to the Jet345 distribution. The difference in fits is folded into the systematic error. These fits are shown in Figure 8.4.

$r_{e}$ is a "raw" fake rate, meaning that the jet sample could contain real electrons, and thus need to be corrected. However, the contamination due to $W$ candidates in our jet samples was investigated by requiring missing $E_{T}\left(\mathscr{E}_{T}\right)<20 \mathrm{GeV}$; the effect of the contamination was negligible. The production cross-section for $W \rightarrow e \nu$ is 


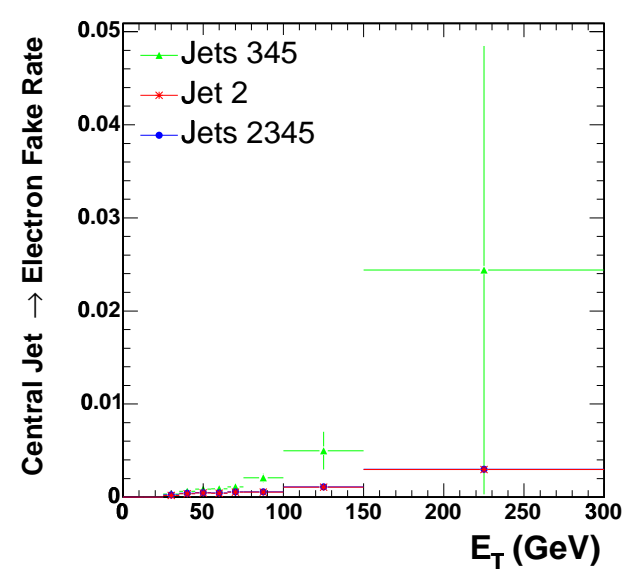

(a)

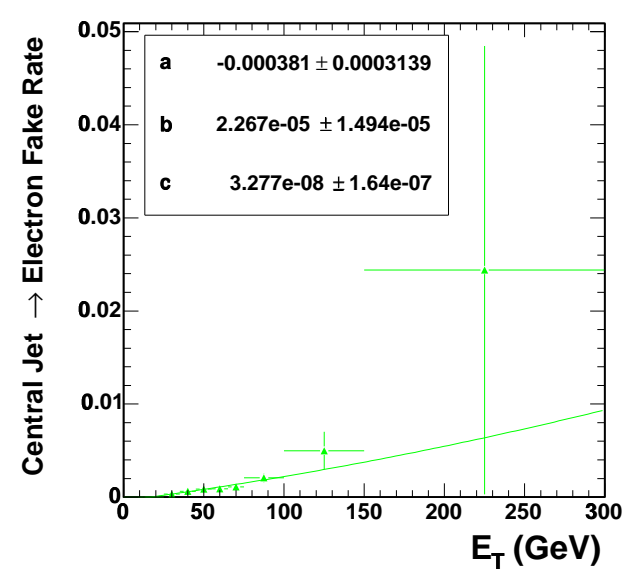

(b)

Figure 8.4: Figure (a) shows the fake rate for Jet2 and Jet345 jets and the combined fake rate. Figure (b) shows the fit for the fake rate of Jet345 jets. The fit function is $r_{e}=a+b \cdot E_{T}+c \cdot E_{T}^{2}$.

about 10 times more than for $Z \rightarrow e e$, so it follows that contamination from $Z$ electrons would also be negligible.

\subsubsection{Systematics}

Based on the $\eta_{d}$ and Jet2 vs Jet345 dependencies on the fake rate, we assign asymmetric systematic errors using the following prescription. Because the Jet2 fake rate does not differ significantly from the combined fake rate, we apply the $63 \%$ systematic error due to the $\eta_{d}$ dependence for the lower limit on the fake rate. For determining the upper limit on the fake rate, if the relative error on the fake rate between the Jet345 fit and the combined fit is greater than $63 \%$, we quote the Jet 345 difference. Else, we assign a $63 \%$ systematic error. Table 8.1 .3 shows the fake rate at various transverse energies. 


\begin{tabular}{|l|c|}
\hline$E_{T}$ & $r_{t} \times 1000$ \\
\hline \hline $30 \mathrm{GeV}$ & $0.28 \pm 0.06($ stat $) \pm 0.17($ sys $)$ \\
\hline $40 \mathrm{GeV}$ & $0.33 \pm 0.07(\text { stat })_{-0.21}^{+0.24}($ sys $)$ \\
\hline $50 \mathrm{GeV}$ & $0.39 \pm 0.08(\text { stat })_{-0.25}^{+0.44}($ sys $)$ \\
\hline $60 \mathrm{GeV}$ & $0.47 \pm 0.10(\text { stat })_{-0.29}^{+0.63}($ sys $)$ \\
\hline $70 \mathrm{GeV}$ & $0.54 \pm 0.11(\text { stat })_{-0.34}^{+0.82}($ sys $)$ \\
\hline $87.5 \mathrm{GeV}$ & $0.68 \pm 0.15(\text { stat })_{-0.43}^{+1.17}($ sys $)$ \\
\hline $125 \mathrm{GeV}$ & $1.04 \pm 0.23(\text { stat })_{-0.66}^{+1.96}($ sys $)$ \\
\hline $225 \mathrm{GeV}$ & $2.35 \pm 0.58(\text { stat })_{-1.48}^{+4.03}($ sys $)$ \\
\hline
\end{tabular}

Table 8.1: Unbiased jet $\rightarrow$ electron fake rate in $E_{T}$ bins.

\subsubsection{Jet $E_{T} \rightarrow$ Electron $E_{T}$ Fragmentation Function}

The energy of the jet that fakes an electron is higher than the electron-like object. This is because the jet energy includes all electromagnetic and hadronic energy in a cone of $\Delta R<0.4$, while the electron energy is restricted to the electromagnetic energy of the cluster's two towers.

When we apply the jet $\rightarrow$ electron fake rate to jet objects in the signal sample, a measure of the energy of the electron that the jet could have faked, or a jet $E_{T}$ $\rightarrow$ electron $E_{T}$ fragmentation function, is needed. To obtain this, the ratio of jet $E_{T}\left(E_{T}^{j}\right)$ to electron $E_{T}\left(E_{T}^{e}\right)$ as a function of jet $E_{T}$ is plotted in Figure 8.5(a). Figure 8.5(b) shows the distribution of the ratio of jet energy $\left(E^{j}\right)$ to electron energy $\left(E^{e}\right)$ as a function of jet energy, and the distribution is similar to Figure 8.5(a). The jet $E_{T} \rightarrow$ electron $E_{T}$ fragmentation function is used because the fake rate is measured as a function of $E_{T}$. It is fit to an exponential function plus a constant, as shown in Figure 8.5(a).

Figures 8.6(a) - (d) show the distributions of the ratios of jet $E_{T}$ to electron $E_{T}$ for all $E_{T}$ and for different ranges of $E_{T}$. The width of the Gaussian distributions shows that the electron $E_{T}$ to jet $E_{T}$ ratios for various $E_{T}$ do not differ much. Thus, 
the fragmentation function of Figure 8.5(a) adequately describes the jet to electron transverse energy relationship.

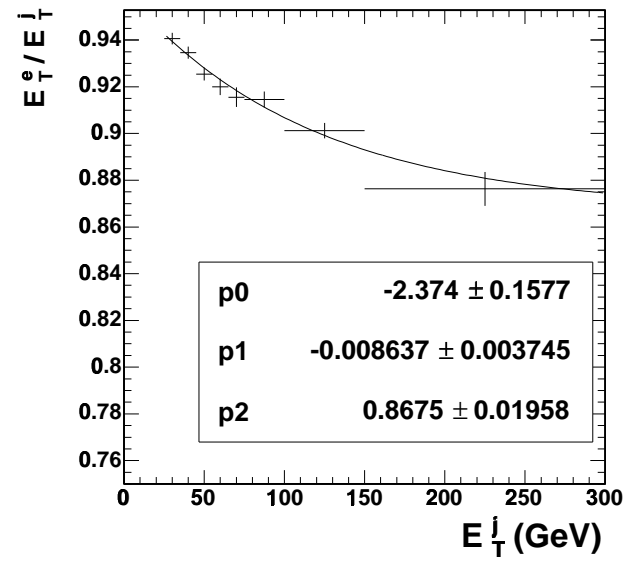

(a)

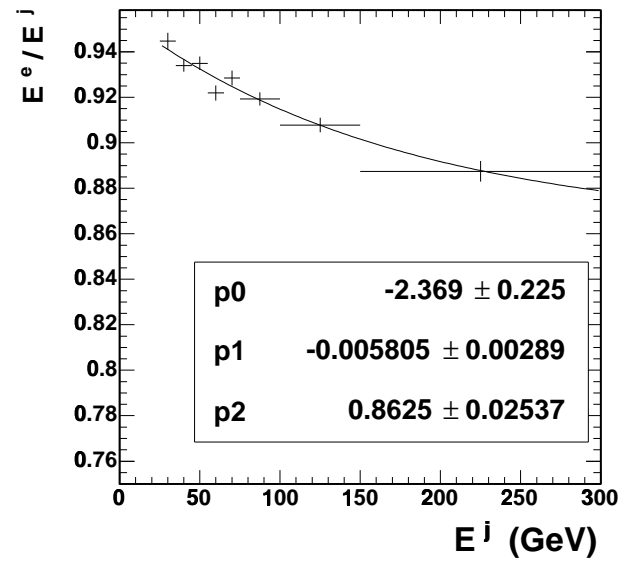

(b)

Figure 8.5: Figure (a) is the $E_{T}^{e} / E_{T}^{j}$ fragmentation function versus jet $E_{T}$. Figure (b) is the $E^{e} / E^{j}$ as a function of jet $\mathrm{E}$. The distributions are fit to $e^{p 0+p 1 \cdot E}+p 2$.

\subsection{Trigger Electromagnetic Object $\rightarrow$ Electron Fake Rate}

The rate at which a jet that fired the trigger will fake an electron is biased by the trigger and thus different than the unbiased jet $\rightarrow$ electron fake rate. To measure this rate, we measure a trigger EM object $\rightarrow$ electron fake rate $\left(r_{t}\right)$.

\subsubsection{Data Samples}

The trigger EM object $\rightarrow$ electron fake rate is measured from the signal dataset, bhel08 and bhel09 data, the events of which are triggered by an electron trigger 


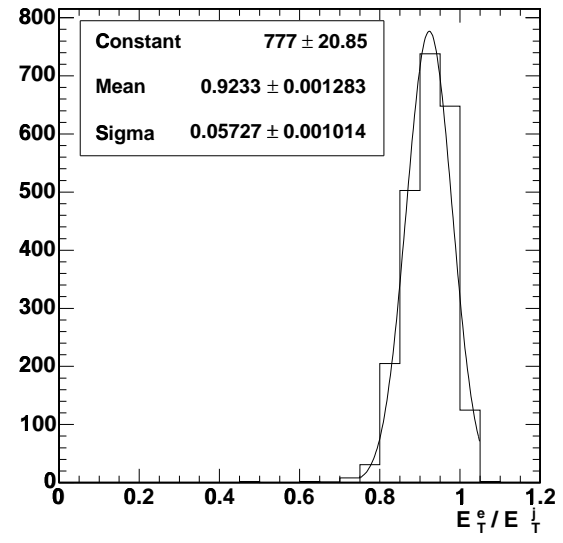

(a)

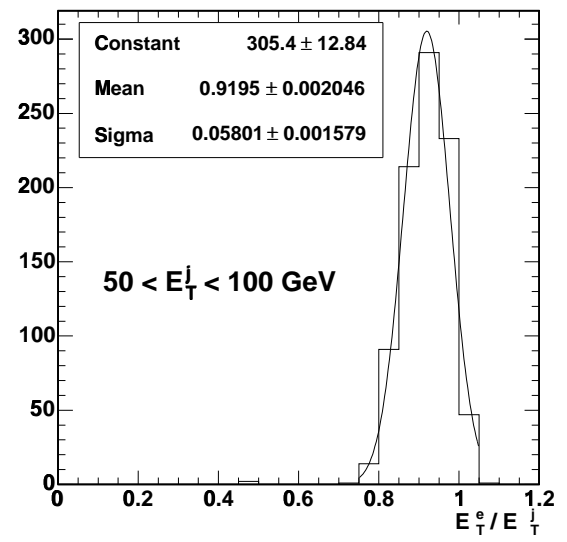

(c)

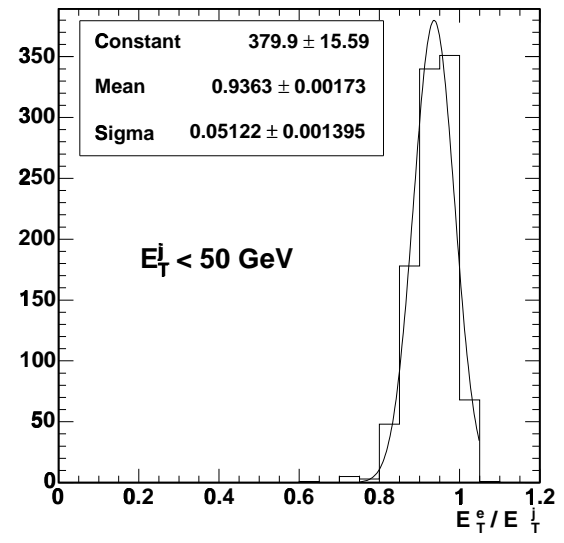

(b)

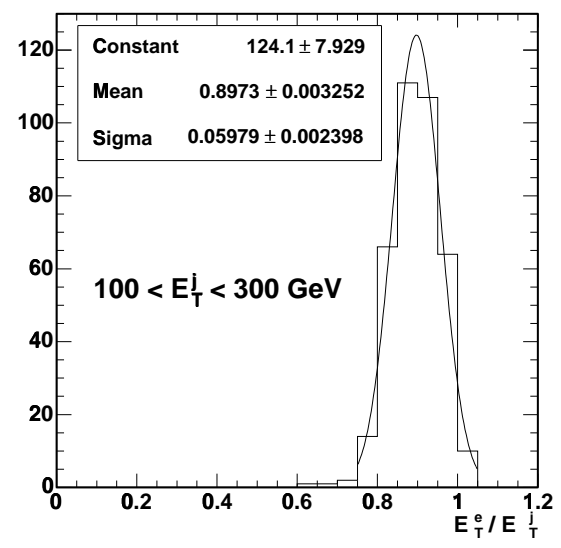

(d)

Figure 8.6: Distributions of $E_{T}^{e} / E_{T}^{j}$ for different ranges of $E_{T}^{j}$. Figure (a) is for all $E_{T}^{j}$. Each is fit to a Gaussian. 
path.

\subsubsection{Measurement}

The majority of real electrons that fire the trigger come from $W \rightarrow e \nu$ and $Z \rightarrow e e$ events. Thus, $r_{t}$ is defined as the rate at which non-W and non-Z trigger EM objects pass the central electron identification criteria. All other objects passing the cuts are classified as "fakes" 1 .

We consider the highest $E_{T}$ central, fiducial electromagnetic object with track $p_{T}>10 \mathrm{GeV}$ in an event. The event must not be a $W \rightarrow e \nu$ or $Z \rightarrow e e$ candidate. To remove $W \rightarrow e \nu$ candidates, we require the event have $\mathbb{E}_{T}<10 \mathrm{GeV}$. The $\mathbb{E}_{T}$ is measured from the vertex of the track associated with the electron. To remove $Z \rightarrow e e$ candidates, we require the event have no more than one EM object, central or plug, passing a loose set of electron selection cuts shown in Table 8.2.

\begin{tabular}{|l|c|c|}
\hline Variable & Central & Plug \\
\hline \hline$|\eta|$ & $<1.0$ & $1.2-2.8$ \\
\hline Fiducial Requirement & standard & - \\
\hline$E_{T}$ & $>25 \mathrm{GeV}$ & $>25 \mathrm{GeV}$ \\
\hline$p_{T}$ & $>10 \mathrm{GeV}$ & - \\
\hline$\frac{\text { Callso }(\Delta R<0.4)}{E_{T}}$ & $<0.1$ & - \\
\hline$\frac{E_{H a d}}{E_{E M}}$ & $<0.055+0.00045 \cdot E$ & $<0.055+0.00045 \cdot E$ \\
\hline $\mathrm{Z}_{\circ}$ & $<60 \mathrm{~cm}$ & - \\
\hline$\frac{E}{p}$ & $<4$ or $E_{T}>100 \mathrm{GeV}$ & - \\
\hline
\end{tabular}

Table 8.2: Loose electron cuts for the $Z \rightarrow$ ee rejection requirement in the trigger EM object $\rightarrow$ electron fake rate measurement. Events that have more than one EM cluster passing these cuts are rejected.

If the event passes the $W \rightarrow e \nu$ and $Z \rightarrow e e$ rejection criteria, we consider

\footnotetext{
${ }^{1}$ The electrons we classify as "fake" may include real electrons from conversions, b-decays, etc
} 
the maximum $E_{T}$ central, fiducial EM cluster and additionally require it pass the following:

- Track $p_{T}>10 \mathrm{GeV}$

- $\frac{\operatorname{CalIso}(\Delta R<0.4)}{E_{T}}<0.1$

- $\frac{E_{H a d}}{E_{E M}}<0.055+0.00045 \cdot E$. The calorimeter isolation and $\frac{E_{H a d}}{E_{E M}}$ cuts are applied because it is a jet that fakes the electron which makes the fake rate a function of jet $E_{T}$. Thus, we must select on EM objects whose energy is isolated and therefore close to the originating jet energy.

- $L_{s h r}<0.2$ or $E_{T}>100 \mathrm{GeV}$ and track $p_{T}>15 \mathrm{GeV} . L_{s h r}$ is required for the ELECTRON_CENTRAL_18 trigger but not at higher $E_{T}$ by the ELECTRON70_L2_JET trigger path. However, because the ELECTRON70_L2_JET trigger path has a higher track $p_{T}$ cut of $15 \mathrm{GeV}$ than the ELECTRON_CENTRAL_18 trigger, we require track $p_{T}>15 \mathrm{GeV}$ for $E_{T}>100 \mathrm{GeV}$.

- $|\Delta Z|<5 \mathrm{~cm}$. A $|\Delta Z|$ cut of $8 \mathrm{~cm}$ is required by the ELECTRON_CENTRAL_18 trigger path. Although it is not a requirement on the ELECTRON70_L2_JET trigger path, we do not loosen it for high $E_{T}$ because this cut is not released at analysis level for high $E_{T}$ objects.

If the EM object passes these criteria, the event is part of the base sample $\left(N_{E M}\right) . N_{e}^{o}$ is defined as the number of events in the base sample which pass the tight central electron selection criteria in Table 4.2. Thus, the trigger EM object $\rightarrow$ electron fake rate $\left(r_{t}\right)$ is defined as $r_{t}=N_{e}^{o} / N_{E M}$.

The fake rate is measured as a function of $E_{T}$. The measured values of $r_{t}$ are given in Table 8.3 and the distribution is shown in Figure 8.7. The fake rate is 
maximum at low $E_{T}$ with a value of approximately $95 \%$. It decreases to and levels off at around $44 \%$ for $E_{T}$ greater than $75 \mathrm{GeV}$. The distribution is fit to a Gaussian plus a constant.

\begin{tabular}{|l|c|}
\hline$E_{T}$ bin & $r_{t} \times 100$ \\
\hline \hline $25<E_{T}<35 \mathrm{GeV}$ & $96.0 \pm 0.1($ stat $)$ \\
\hline $35<E_{T}<45 \mathrm{GeV}$ & $92.1 \pm 0.1($ stat $)$ \\
\hline $45<E_{T}<55 \mathrm{GeV}$ & $77.6 \pm 0.4($ stat $)$ \\
\hline $55<E_{T}<65 \mathrm{GeV}$ & $64.4 \pm 0.8($ stat $)$ \\
\hline $65<E_{T}<75 \mathrm{GeV}$ & $55.9 \pm 1.2($ stat $)$ \\
\hline $75<E_{T}<100 \mathrm{GeV}$ & $44.8 \pm 1.3($ stat $)$ \\
\hline $100<E_{T}<150 \mathrm{GeV}$ & $43.7 \pm 1.8($ stat $)$ \\
\hline$E_{T}>150 \mathrm{GeV}$ & $48.1 \pm 5.6($ stat $)$ \\
\hline
\end{tabular}

Table 8.3: Trigger EM object $\rightarrow$ electron fake rate in $E_{T}$ bins.

Additionally, we investigate the dependence of fake rate on detector $\eta$. Figure 8.8 shows the average fake rate as a function of $\eta_{d}$. Because most of the EM objects have low $E_{T}$, where the fake rate is high, the weighted average fake rate is $92.7 \%$. There is no appearant $\eta_{d}$ dependence on the fake rate, and the standard deviation is $\sigma \sim 1.3$, or $1.4 \%$ relative to the average.

Figure 8.9 shows that the trigger EM object $\rightarrow$ electron fake rate is stable in time.

\subsubsection{Measurement Uncertainty}

The statistical error at low $E_{T}$ is negligible. For $E_{T}$ between 65 and $150 \mathrm{GeV}$, we apply a $1.5 \%$ absolute statistical error. For $E_{T}$ greater than 150 , a $5.6 \%$ absolute statistical error is used. To account for the small spread in $\eta_{d}$, we apply a $1.4 \%$ relative error to the fake rate for all values of $E_{T}$. We studied the "contamination" of the background sample due to real electrons from $W \rightarrow e \nu$ and $Z \rightarrow e e$ events 


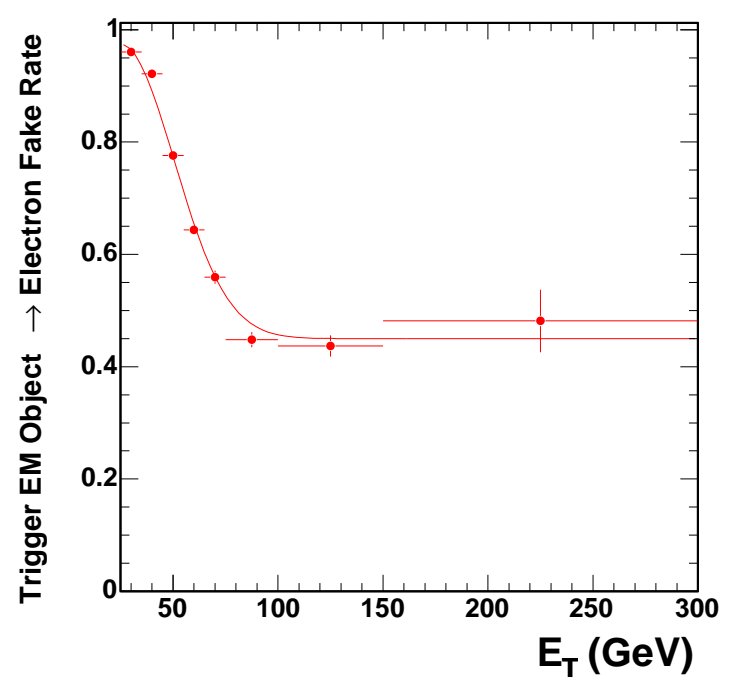

Figure 8.7: Trigger EM object $\rightarrow$ electron fake rate as a function of $E_{T}$. The errors shown are statistical. The distribution is fit to a Gaussian plus a constant. The fit parameters are 0.52 for Gaussian norm, $25 \mathrm{GeV}$ for Gaussian mean, 25.5 $\mathrm{GeV}$ for Gaussian sigma, and 0.45 for the constant.

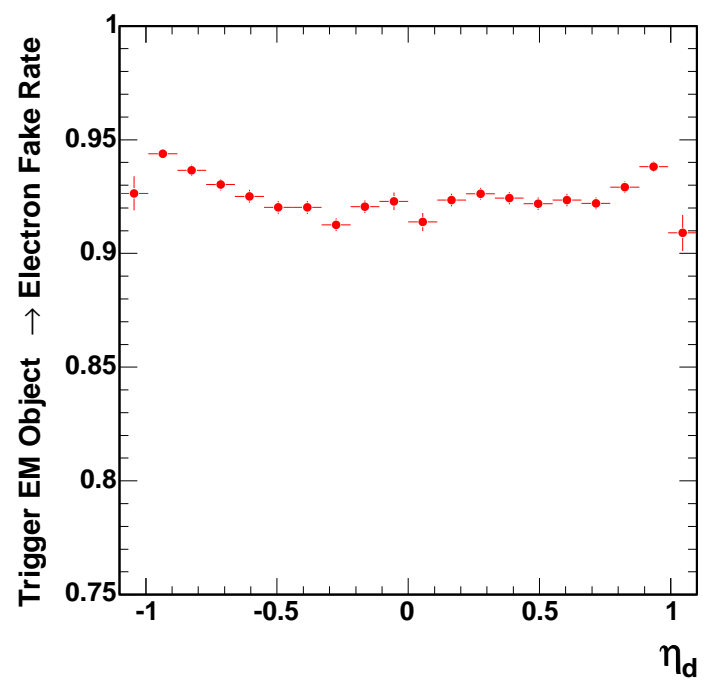

Figure 8.8: Trigger EM object $\rightarrow$ electron fake rate as a function of detector $\eta$. The errors shown are statistical. 


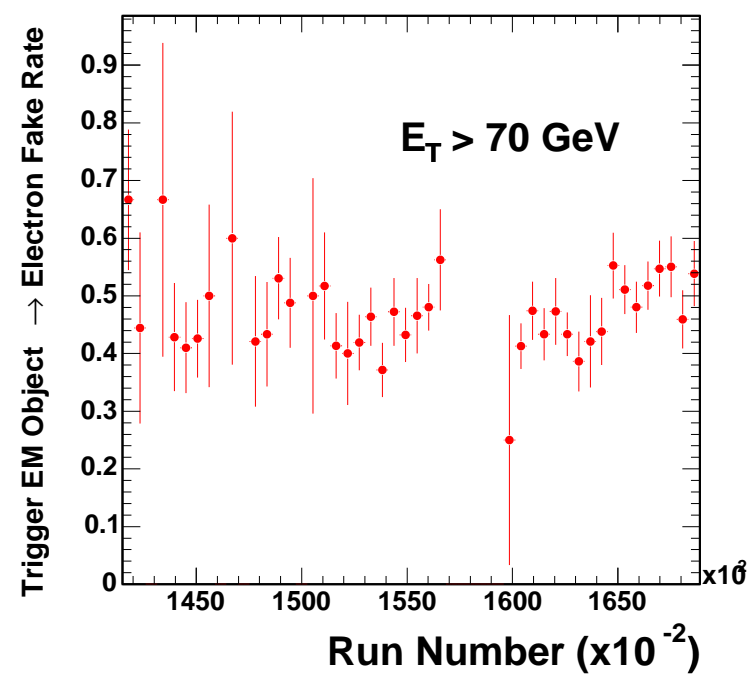

Figure 8.9: Trigger EM object $\rightarrow$ electron fake rate as a function of run number. The errors shown are statistical.

and found the effect to be negligible.

\subsection{Unbiased Central Jet $\rightarrow$ Photon Fake Rate}

We now describe the measurement of the rate of unbiased central jets to be misidentified as photons. This study was documented in [20] and is reproduced with some modifications here. Similar to the unbiased jet $\rightarrow$ electron fake rate (Section 8.1), the raw fake rate is simply defined by the fraction of non-trigger jets that pass the central photon identification cuts.

The data from which we measured the electron fake rate did not contain significant prompt electron contamination. This is not the case for photons. The data contain true prompt photons that are classified as jets, and the photons pass the selection criteria with high efficiency. Thus, the raw fake rate overestimates the probability of QCD jets faking photons. The prompt photon fraction of the jet 
candidates is measured on a statistical basis by exploiting the difference between the conversion probability of true single photons and multiple collinear photons in a QCD jet. The Central Pre-Radiator (CPR) detector is used to identify candidates that convert upstream of the CPR.

\subsubsection{Raw Fake Rate}

\section{Data Samples}

As for the unbiased jet $\rightarrow$ electron fake rate, QCD data triggered on the JET_20, JET_50, JET_70, and JET_100 trigger paths are used to obtain a sample of unbiased jets. The highest- $E_{T}$ jet in the event is assumed to be the trigger jet and is excluded from the measurement. All other jets in the event in a given $E_{T}$ bin are used for measuring the jet $\rightarrow \gamma$ fake rate.

\section{Measurement}

The raw fake rate is defined as the probability that a jet matches a photon candidate. We select unbiased, central jets with $E_{T}>25 \mathrm{GeV}$ and $Z_{v t x}<60 \mathrm{~cm}$, reconstructed with a cone of radius $\mathrm{R}=0.4$. Photon candidates are selected using the standard tight photon cuts listed in Table 4.3. A jet is considered to also be a photon candidate if it matches the photon with $\Delta R<0.15$, where $\Delta R=\sqrt{\Delta \eta^{2}+\Delta \phi^{2}}$. Figure 8.10 shows the $\Delta R$ distribution between jets and photons. Thus, for $N_{j}$ jets considered, if we find $N_{\gamma}^{o}$ photon candidate matches, the raw jet $\rightarrow$ photon fake rate is $r_{\text {raw }}=N_{\gamma}^{o} / N_{j}$. Table 8.4 and Figure 8.11 shows the raw fake rates measured in $E_{T}$ bins. Because the statistics for photon fake rate measurement are more limited, wider bins are used than for the other fake rate measurements. 


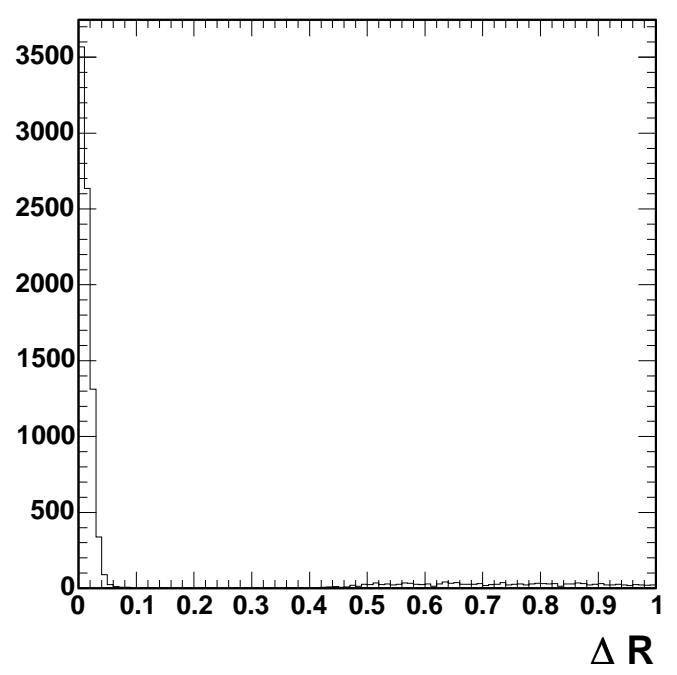

Figure 8.10: $\Delta R$ between jets and photon candidates. We use a cut of $\Delta R<0.15$ to match jets to photons.

\begin{tabular}{|l|c|c|c|c|}
\hline$E_{T}$ bin & $N_{j}$ & $N_{\gamma}^{o}$ & $r_{\text {raw }} \times 1000$ & $\delta r_{\text {raw }} / r_{\text {raw }}(\%)$ \\
\hline \hline $25<E_{T}<39 \mathrm{GeV}$ & 1851450 & 1563 & $0.84 \pm 0.02$ & 2.5 \\
\hline $39<E_{T}<70 \mathrm{GeV}$ & 2042020 & 1403 & $0.69 \pm 0.02$ & 2.7 \\
\hline $70<E_{T}<200 \mathrm{GeV}$ & 1034450 & 640 & $0.62 \pm 0.02$ & 4.0 \\
\hline
\end{tabular}

Table 8.4: Raw $j \rightarrow \gamma$ fake rate.

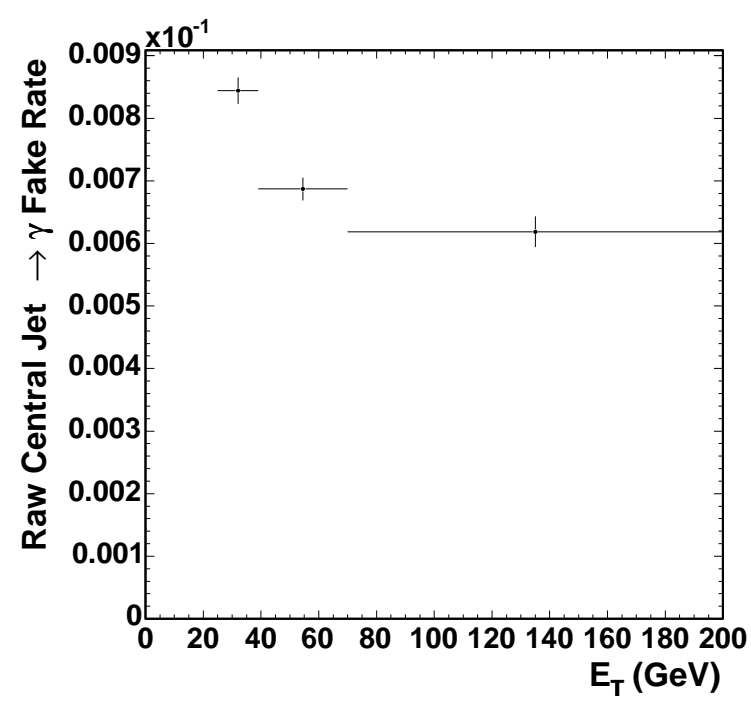

Figure 8.11: Raw $j \rightarrow \gamma$ fake rate. 


\subsubsection{Prompt Photon Subtraction}

The fake rate is labeled "raw" because some of the jet candidates are true prompt photons. The fake rate needs to be corrected for the prompt photon "contamination". We have measured $r_{\text {raw }}$ :

$$
r_{\text {raw }}=\frac{N_{\gamma}^{\circ}}{N_{j}}=\frac{N_{\gamma}^{\text {true }}+N_{\gamma}^{\text {fake }}}{N_{j}}
$$

where $N_{\gamma}^{\text {true }}$ is the number of true prompt photons and $N_{\gamma}^{f a k e}$ is the number of faked photons in the sample of observed photons, $N_{\gamma}^{\circ}$. We measure the fraction of true prompt photons $\left(f_{\gamma}\right)$ in the sample of photon fake candidates, $N_{\gamma}^{\circ}$, by using the Central Pre-Radiator (CPR) detector to measure the fraction of photon candidates which deposit a conversion signal. $f_{\gamma}$, which is given by $f_{\gamma}=N_{\gamma}^{\text {true }} / N_{\gamma}^{\circ}$, is then used to determine the true central jet $\rightarrow$ photon fake rate $\left(r_{\gamma}\right)$ by subtracting the number of true photon candidates from the number of observed photon fakes. Thus $N_{\gamma}^{\text {true }}$ is given by $N_{\gamma}^{\text {true }}=f_{\gamma} N_{\gamma}^{\circ}$ and the true fake rate is given by :

$$
r_{\gamma}=\frac{N_{\gamma}^{\text {true }}+N_{\gamma}^{\text {fake }}-f_{\gamma} N_{\gamma}^{\circ}}{N_{j}}=r_{\text {raw }}-f_{\gamma} r_{\text {raw }}=r_{\text {raw }}\left(1-f_{\gamma}\right)
$$

\section{Data Samples}

To study the CPR detector response to single minimum-ionizing particles, we use muons from a cosmic-ray sample and from the inclusive $W \rightarrow \mu \nu$ sample. Electrons from the inclusive $W \rightarrow e \nu$ sample are used to provide additional information about the CPR. Additionally, the $W \rightarrow \mu \nu$ sample is used for studying the underlying event fluctuations in the CPR detector. Finally, for some photon studies, we use data that were selected on a photon trigger, specifically PHOTON_25 data. 


\section{Prompt Photon Fraction Measurement Using the CPR}

The CPR detector consists of a set of chambers placed between the solenoid coil and the electromagnetic calorimeter. The CPR detects the charge signal of electrons produced by those photons that convert in the coil. We classify the photon candidates as being either single, prompt photons or collinear photons from $\pi^{\circ}$ decays ${ }^{2}$. Different conversion probabilities are expected for these categories. The observed average conversion rate allows us to infer the relative fractions of the two categories.

Figure 8.12 shows the CPR charge $(\mathrm{Q})$ detected for high $p_{T}$ muons and photon candidates from the PHOTON_25 sample. Muons are obtained from a very clean cosmic ray sample (with no concurrent $p \bar{p}$ collision) and from the inclusive $W \rightarrow \mu \nu$ sample. Figure 8.13 shows the predicted dimuon CPR $Q$ distribution, calculated by convoluting the cosmic ray CPR $Q$ distribution with itself. The latter distribution serves as an illustration of the expected CPR $Q$ distribution from a pair of conversion electrons.

We define a $\gamma \rightarrow e e$ conversion as one where $\mathrm{CPR} Q>500 \mathrm{fC}$ is detected. The dimuon CPR $Q$ distribution indicates that this cut is $>99 \%$ efficient. We measure the CPR detection efficiency using cosmic rays with $Q>100 \mathrm{fC}$, which has the same efficiency for the single muon signal.

Having defined an observed photon conversion, we must take into account effects that cause differences between the observed number of photon conversions and the true number of photon conversions. The true number of photon conversions will be used to extract the prompt photon fraction. CPR detector efficiency and fluctuations due to underlying event are two effects considered in the calculation of the

\footnotetext{
${ }^{2}$ The majority of the pairs of photons are from single $\pi^{\circ}$ decays. However, a portion may be from other meson decays such as neutral kaon decay to two $\pi^{\circ}$ which then decay to photons. These sources of background photons are also taken into account in the analysis.
} 


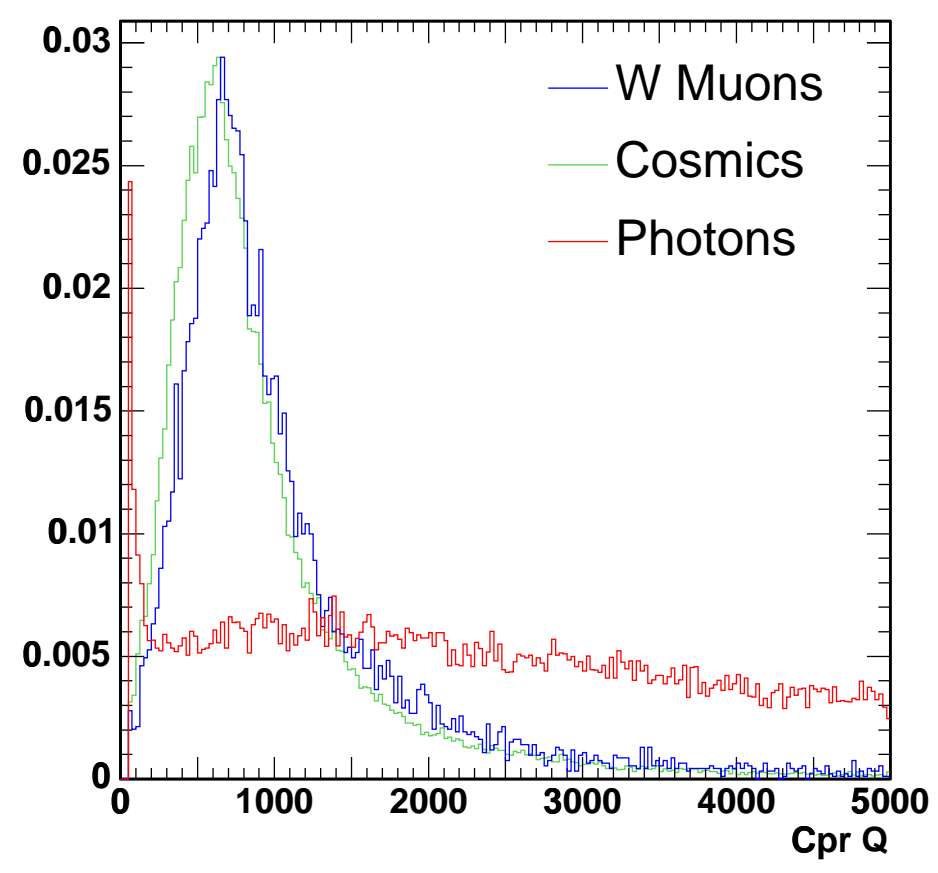

Figure 8.12: CPR $Q$ distributions for cosmic ray and $W \rightarrow \mu \nu$ muons and the photon candidates. The units of $\mathrm{Q}$ are $\mathrm{fC}$.

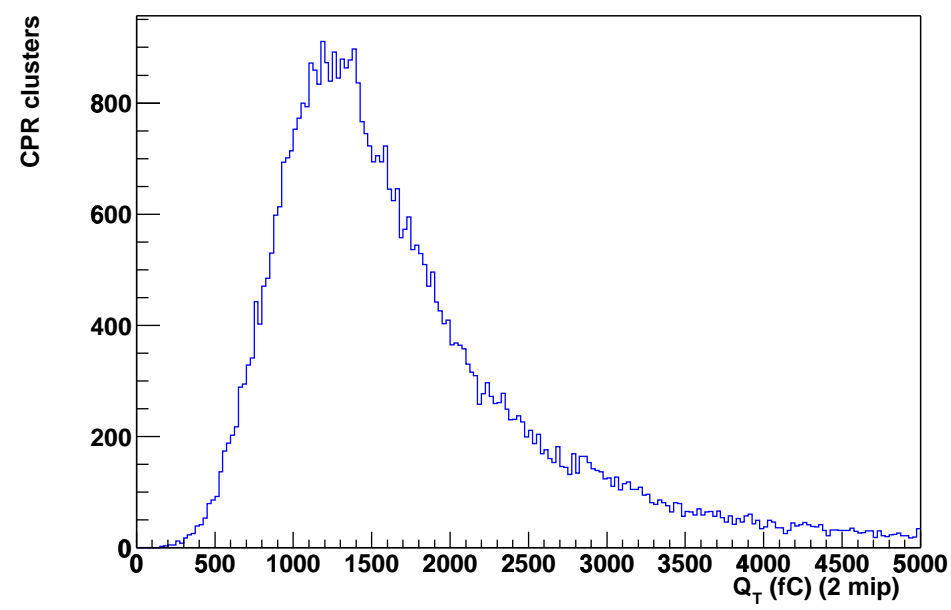

Figure 8.13: Predicted distribution of $\mathrm{CPR} Q_{T}=Q \sin \theta$ for two collinear minimum-ionizing particles [18]. 
true number of photon conversions.

\section{CPR Detection Efficiency}

The probability of detecting photon conversions depends on CPR detection efficiency. Inefficiency in the CPR will result in some photon conversions being counted as non-conversions. Thus, we measure the CPR detection efficiency and later take it into account as part of the prompt photon fraction measurement. An upper estimate of the efficiency is provided by electrons from the $W \rightarrow e \nu$ sample. The CPR $Q$ signal for electrons is significantly larger than that for muons or photon candidates (see Figure 8.14) due to the presence of a "photon-cloud" near the electron. Since the photon candidate signal in the CPR is intermediate between the muon and electron signal, we average the CPR detection efficiency measurements using muons and electrons to obtain the conversion detection efficiency, and quote half their difference as the systematic error.

Some regions of the central detector are not well instrumented by the CPR. The measurement is restricted to those regions with good CPR coverage. To determine the fiducial region of the CPR, the muon detection efficiency is studied as a function of the local $X$ (transverse) and $Z$ (longitudinal) coordinates, and as a function of CPR barrel and module. Figure 8.15 shows the muon detection efficiency for different barrels and modules. The efficiency shown is for "track-seeded" CPR hits, which simply means that the CPR cluster is formed using information about the position of the muon track. Modules 5 and 20 in the east barrel and modules 9 , 19 and 22 in the west barrel are identified as low-efficiency modules. Thus, photon candidates from these modules are excluded in the measurement of the prompt photon fraction. Figure 8.16 shows the dependence of the efficiency on local $X$ and 


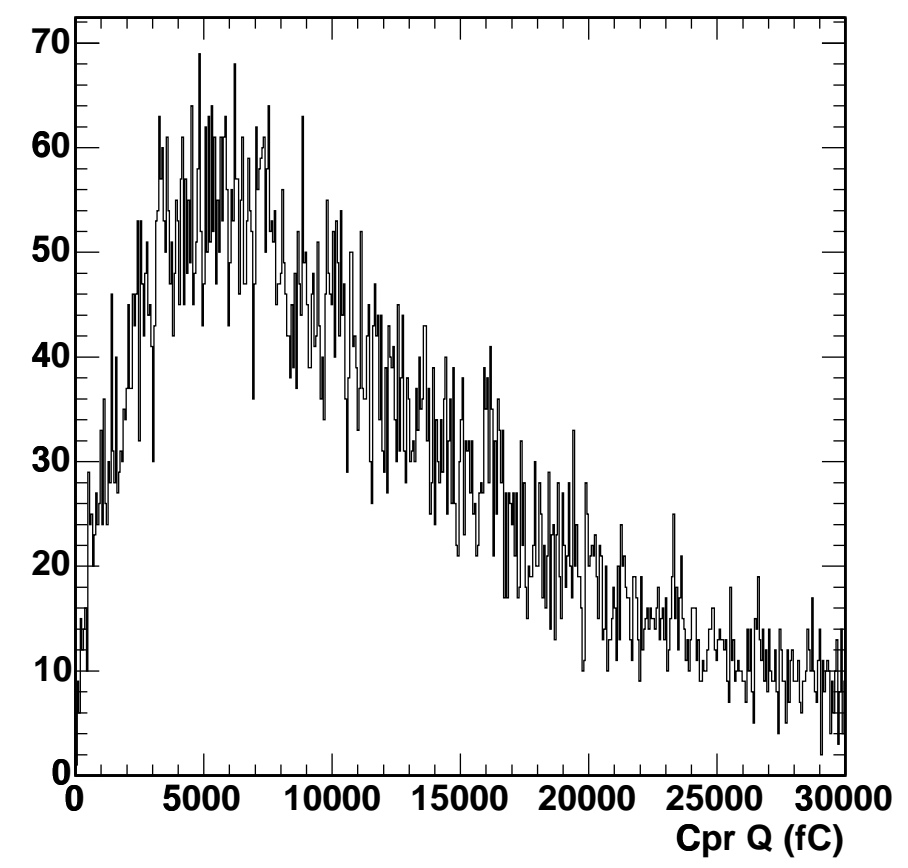

Figure 8.14: CPR $Q$ distribution for electrons from $W \rightarrow e \nu$ candidates. Note the difference in the $Q$ scale between electrons and the photon candidates (Figure 8.12). 
local $Z$. On the basis of these plots, we define the CPR fiducial region using the CES cluster to be

- $\left|X_{C E S}\right|<16 \mathrm{~cm}$

- $10 \mathrm{~cm}<\left|Z_{C E S}\right|<110 \mathrm{~cm}$ or $130 \mathrm{~cm}<\left|Z_{C E S}\right|<210 \mathrm{~cm}$

The unbiased (not track-seeded) CPR detection efficiency measured using muons located in the CPR fiducial regions of the detector is $\epsilon_{C P R}^{\mu}=(97.6 \pm 0.1) \%$ and the same measurement using electrons is $\epsilon_{C P R}^{e}=(99.01 \pm 0.04) \%$. The average is used $\epsilon_{C P R}=(98.3 \pm 0.5) \%$ as the conversion detection efficiency.

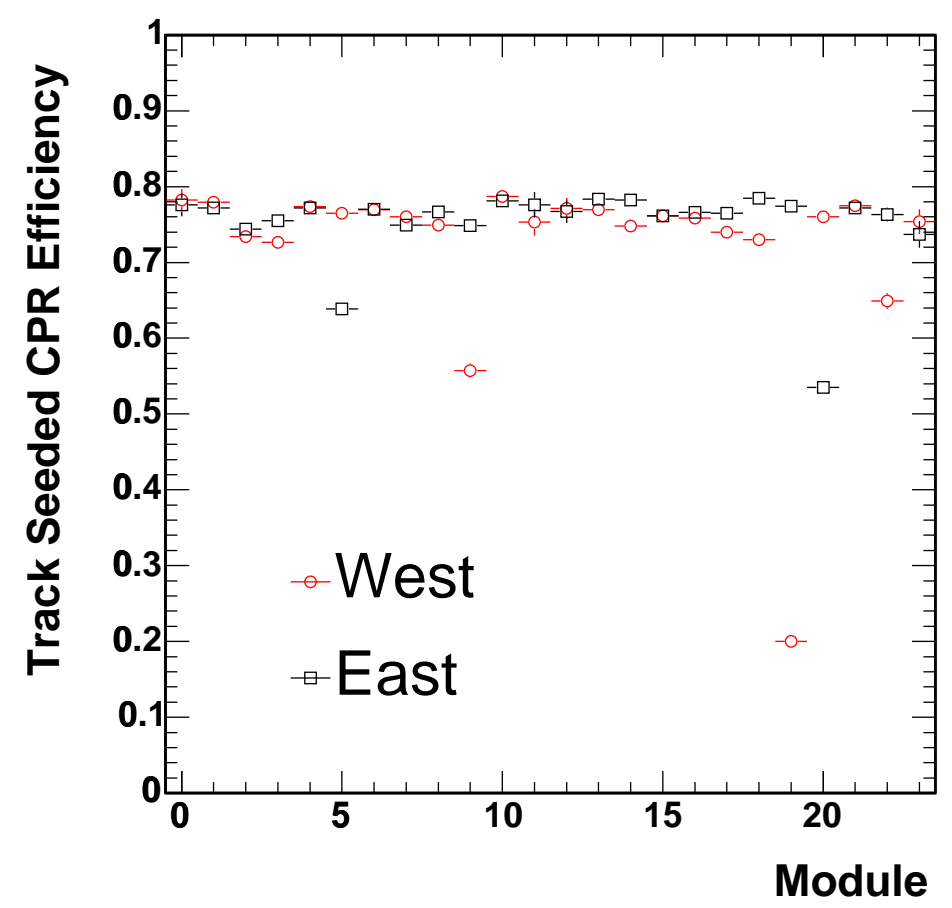

Figure 8.15: Dependence of CPR detector efficiency on barrel and module. 


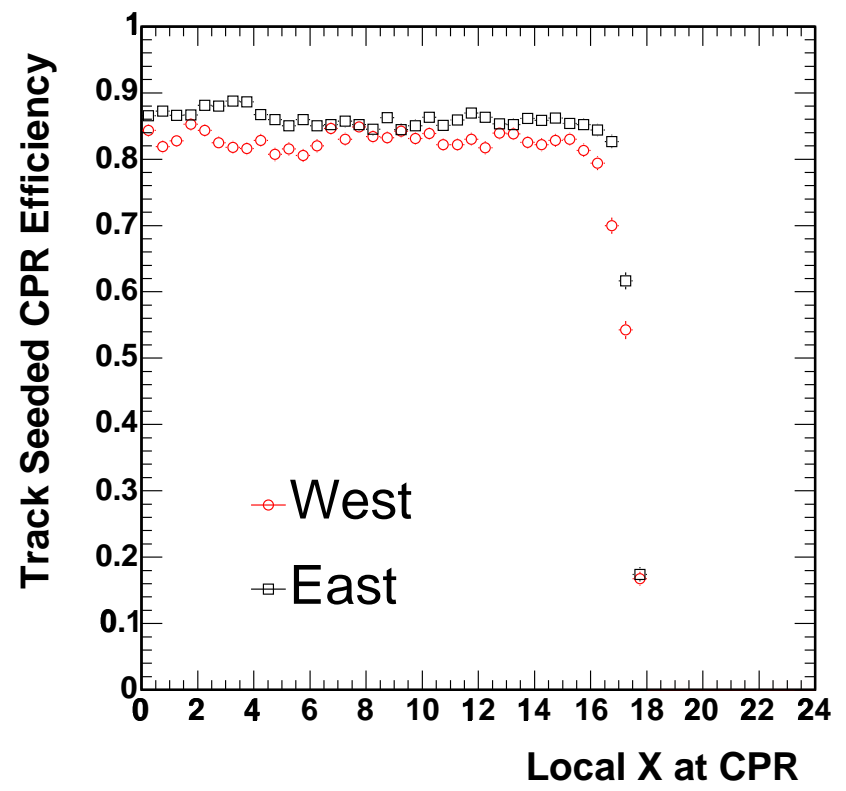

(a)

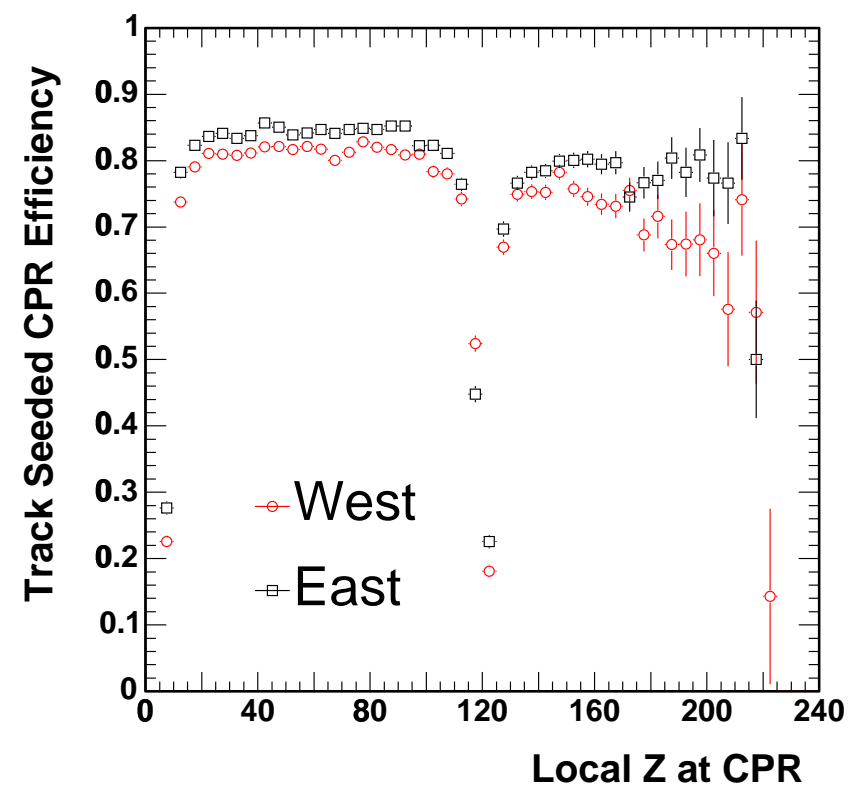

(b)

Figure 8.16: Dependence of CPR detector efficiency on local $X$ (a) and local $Z$ (b). 


\section{Underlying Event Fluctuations in the CPR}

We are measuring the rate at which the photon candidates deposit a conversion signal in the CPR. Sometimes an underlying event fluctuation, or electronic noise, creates a CPR signal which is falsely associated with a non-converted photon candidate, mislabeling it as a converted candidate. The fractional rate of this misassignment is denoted by $F_{U E}$.

Figure 8.17 shows the distribution of $\Delta X$ between the local $X$ coordinate of all CPR clusters and the local $X$ coordinate of the CES cluster associated with the photon candidate (in the same module). We see the true conversion signal as the peak at $\Delta X \sim 0$, with a broad distribution from all other CPR clusters not produced by the photon candidate. As a validation of the peak width, we look at the position resolution of the CES and CPR detectors. We have independently measured the CPR position resolution using muons to be $\delta X \sim 0.85 \mathrm{~cm}$ (see Figure 8.18) and the CES position resolution using electrons to be $\delta X \sim 1 \mathrm{~cm}$. Hence we expect the $\Delta X$ distribution for true conversions to have an r.m.s. $\sim 1.5 \mathrm{~cm}$, consistent with the peak visible in Figure 8.17.

Figure 8.18 shows the distribution of $\Delta X$ between the local $X$ coordinate of the muon track from $W \rightarrow \mu \nu$ events and all CPR clusters in the same module. The sidebands of this distribution are primarily due to underlying event fluctuations. We expect the underlying event contribution would be greater around real activity in the event. Thus, we estimate $F_{U E}$ by measuring the rate (per muon candidate) of observing at least one CPR cluster with $Q>500 \mathrm{fC}$ in each of three $\Delta X$ windows: $5 \mathrm{~cm}<|\Delta X|<10 \mathrm{~cm}, 10 \mathrm{~cm}<|\Delta X|<15 \mathrm{~cm}$, and $15 \mathrm{~cm}<|\Delta X|<20 \mathrm{~cm}$. We measure the rates to be $(7.3 \pm 0.2) \%,(5.4 \pm 0.2) \%$ and $(4.2 \pm 0.2) \%$ in these windows respectively. Then we extrapolate the underlying event in the vicinity of the muon 


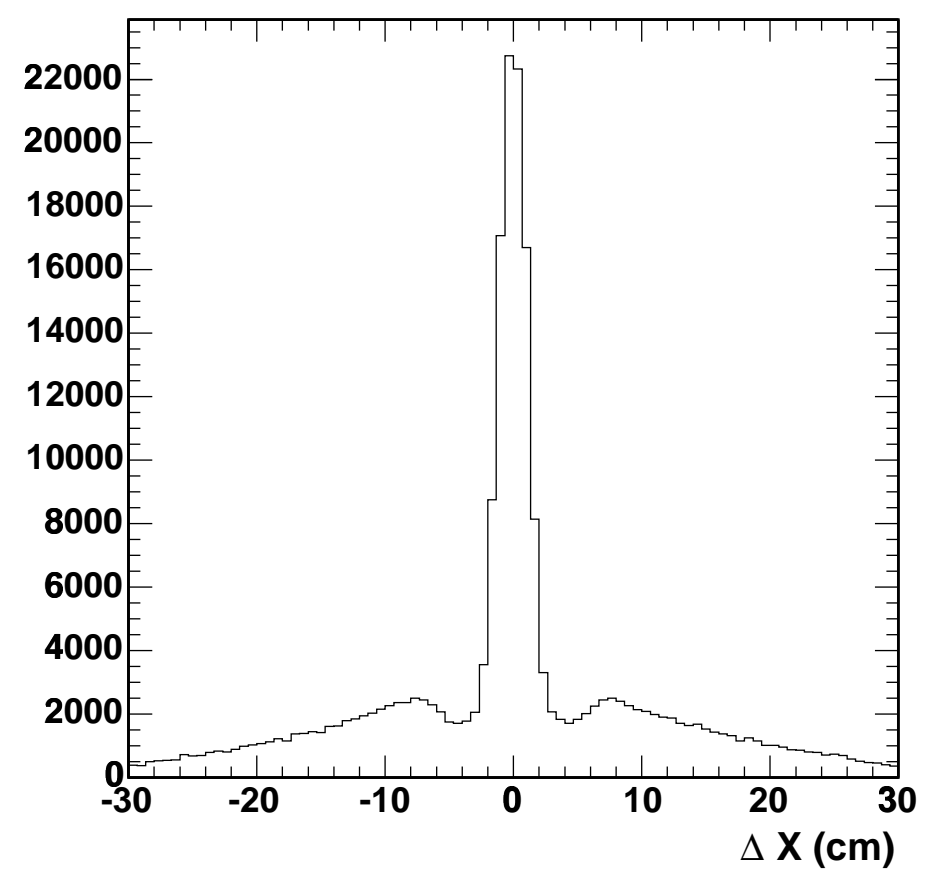

Figure 8.17: Distribution of $\Delta X$ between the local $X$ coordinate of the photon candidate's CES cluster and all CPR clusters in the same half-module. 
track by extrapolating into the $|\Delta X|<5 \mathrm{~cm}$ bin. The first two bins are used in the extrapolation to obtain $F_{U E}=(9.2 \pm 0.7) \%$. The uncertainty includes statistical error $(0.7 \%)$ and systematic contributions from possible non-linear extrapolation $(0.2 \%)$

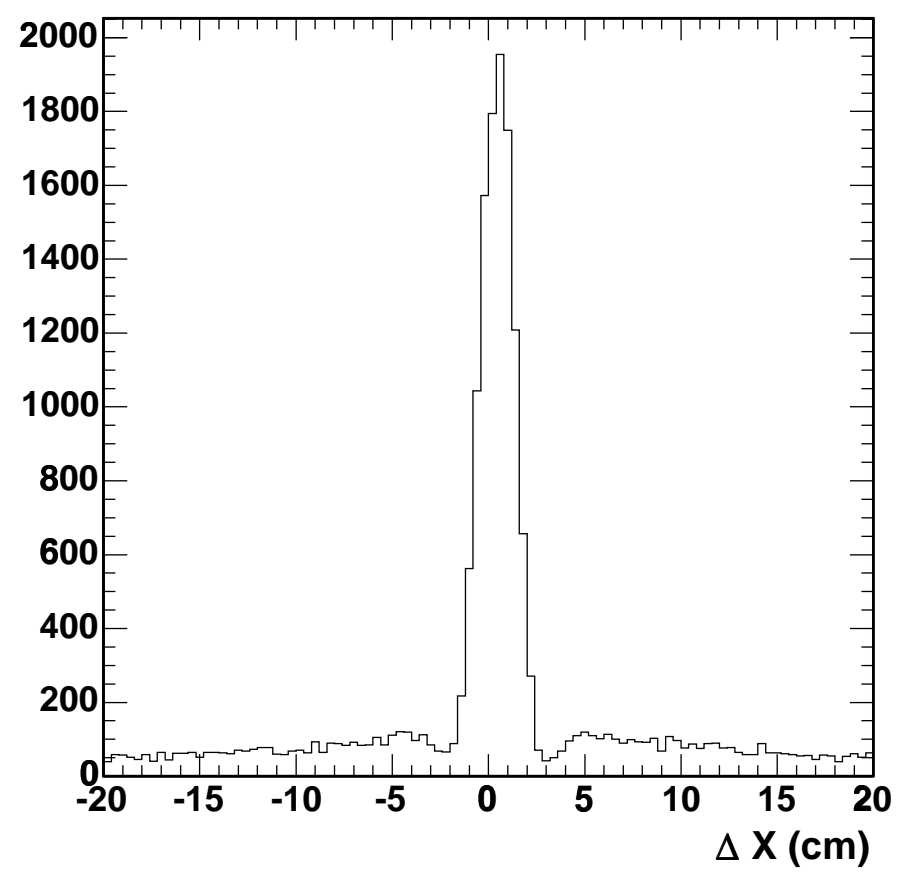

Figure 8.18: Distribution of $\Delta X$ between the local $X$ coordinate of the muon track from $W \rightarrow \mu \nu$ sample and all CPR clusters in the same module.

\section{True Conversion Rate}

The effects described above cause migration of non-converted photon candidates to the converted category (underlying event) and vice versa (CPR detection inefficiency). By correcting for these effects, we can use the observed conversion rate to extract the true conversion rate.

In a given $E_{T}$ bin, of the observed number of photon candidates $\left(N_{\gamma}^{o}\right)$, we de- 
fine $N_{c}^{o}$ and $N_{n c}^{o}$ as the number of candidates with and without a conversion signal in the CPR, respectively. The following equations relate the number of observed conversions to the number of true conversions $\left(N_{c}\right)$, the number of true non-conversions $\left(N_{n c}\right)$, the number of non-conversions mis-assigned as conversions due to $F_{U E}\left(N_{n c \rightarrow c}^{U E}\right)$, and the number of conversions that are not detected due to CPR detection inefficiency $\left(N_{c \rightarrow n c}^{C P R \text { ineff }}\right)[21]$ :

$$
\begin{gathered}
N_{c}^{o}=N_{c}+N_{n c \rightarrow c}^{U E}-N_{c \rightarrow n c}^{C P R \text { ineff }} \\
N_{n c}^{o}=N_{n c}-N_{n c \rightarrow c}^{U E}+N_{c \rightarrow n c}^{C P R} \text { ineff }
\end{gathered}
$$

where we compute the number of events migrating from the non-conversion to the conversion category (due to underlying event) as:

$$
N_{n c \rightarrow c}^{U E}=F_{U E} \cdot N_{n c}
$$

and the number of events migrating from the conversion to the non-conversion category (due to CPR inefficiency) as

$$
N_{c \rightarrow n c}^{C P R \text { ineff }}=\left(1-\epsilon_{C P R}\right) \cdot\left(1-F_{U E}\right) \cdot N_{c} .
$$

Using these relations and some algebra, the true number of conversions and nonconversions can be written as:

$$
\begin{aligned}
N_{c} & =\frac{a \cdot N_{c}^{o}-d \cdot N_{n c}^{o}}{a \cdot c-b \cdot d} \\
N_{n c} & =\frac{c \cdot N_{n c}^{o}-b \cdot N_{c}^{o}}{a \cdot c-b \cdot d}
\end{aligned}
$$


where the following variables are defined

$$
\begin{aligned}
& a=1-F_{U E} \\
& b=\left(1-F_{U E}\right) \cdot\left(1-\epsilon_{C P R}\right) \\
& c=1-b \\
& d=1-a
\end{aligned}
$$

Table 8.5 shows the observed number of converted and non-converted photon candidates and the corresponding true numbers extracted using the relations above.

\begin{tabular}{|l|c|c|c|c|}
\hline$E_{T}$ bin & $N_{c}^{o}$ & $N_{n c}^{o}$ & $N_{c}$ & $N_{n c}$ \\
\hline \hline $25<E_{T}<39 \mathrm{GeV}$ & 705 & 208 & 695.8 & 217.2 \\
\hline $39<E_{T}<70 \mathrm{GeV}$ & 625 & 183 & 616.9 & 191.1 \\
\hline $70<E_{T}<200 \mathrm{GeV}$ & 299 & 101 & 293.8 & 106.2 \\
\hline
\end{tabular}

Table 8.5: Observed and true conversion rates for photon candidates.

\section{Expected Conversion Probabilities}

We now describe how the number of photon conversions are used to measure the fraction of true prompt photons in the jet sample. This procedure is also described in detail in reference [21]. From reference [22], the Run II radiation lengths for electrons upstream of the CPR is $X_{0}^{e}=1.072 \pm 0.018 X_{0}$ (compared to $1.075 \pm 0.023 X_{0}$ in Run I). The conversion length for photons is $7 / 9$ the radiation length for electrons. Therefore $X_{0}^{\gamma}=\frac{7}{9} X_{o}^{e}=0.834 \pm 0.014 X_{0}$.

For an oblique angle of incidence, the thickness of material increases as $\operatorname{cosec} \theta$. The average value of this correction factor has been computed in Run 1, for prompt 
photons and QCD background (primarily $\pi^{\circ}$ events ${ }^{3}$ ):

- $<F>_{\gamma}^{c o r r}=1.1578 \pm 0.0005$

- $<F>_{\pi}^{c o r r}=1.1697 \pm 0.0010$

This gives us the average radiator thickness (using Run 1 correction):

- $<X_{0}^{\gamma}>=<F>_{\gamma}^{\text {corr }} \cdot X_{0}^{\gamma}=0.966 \pm 0.016 X_{0}$

- $<X_{0}^{\pi}>=<F>_{\pi}^{\text {corr }} \cdot X_{0}^{\gamma}=0.976 \pm 0.016 X_{0}$

Therefore the average probability of conversion for a single, prompt photon is:

- $P_{n c}^{\gamma}=e^{-<X_{0}^{\gamma}>}=0.3806 \pm 0.0061$

- $P_{c}^{\gamma}=1-P_{n c}^{\gamma}=0.6194 \pm 0.0061$

The QCD background is predominantly $\pi^{0} / \eta \rightarrow \gamma \gamma$, and a conversion signal is obtained if any of the decay photons convert. The effective number of photons differs from two in an $E_{T}$-dependent way due to two effects: other particle decays produce more than two photons, and some of the photons are not collinear at low $E_{T}{ }^{4}$. We define $m_{\gamma}$ as the collinear photon multiplicity, calculated in the Run 1 analysis [23]. Using the collinear photon multiplicity, the probability of QCD background converting upstream of the CPR is:

- $P_{n c}^{\pi}=e^{-m_{\gamma}<X_{0}^{\pi}>}=0.1420 \pm 0.0045\left(\right.$ for $m_{\gamma}=2$ )

- $P_{c}^{\pi}=1-P_{n c}^{\pi}=0.8580 \pm 0.0045\left(\right.$ for $\left.m_{\gamma}=2\right)$

\footnotetext{
${ }^{3}$ Because the primary source of QCD photon backgrounds are from $\pi^{\circ}$, we refer to the fraction of QCD events in the raw fake rate as $f_{\pi}$ even though decays from other particles contribute.

${ }^{4}$ Examples of particle decays to photons are $K^{\circ} \rightarrow \pi^{\circ} \pi^{\circ} \rightarrow \gamma \gamma \gamma \gamma$ and $\eta \rightarrow \pi^{\circ} \pi^{\circ} \pi^{\circ} \rightarrow \gamma \gamma \gamma \gamma \gamma \gamma$.
} 
Back-splash from the electromagnetic shower in the calorimeter enhances the effective conversion probability by creating a matched CPR cluster. This probability $\left(F_{b}\right)$ is dependent on the electromagnetic shower energy and was estimated in Run 1 photon analyses [23] to be $F_{b}=0.00074 \cdot E$. Since the detector configuration has not changed, we use the same back-splash probability in Run 2 .

The number of observed photon candidates $N_{\gamma}^{o}$ can now be decomposed into the true number of prompt photons $\left(N_{\gamma}\right)$ and the number due to meson decays that are primarily $\pi^{\circ}$ decays $\left(N_{\pi}\right)$ :

$$
\begin{aligned}
N_{c} & =P_{c}^{\gamma} \cdot N_{\gamma}+P_{c}^{\pi} \cdot N_{\pi} \\
N_{n c} & =P_{n c}^{\gamma} \cdot N_{\gamma}+P_{n c}^{\pi} \cdot N_{\pi}
\end{aligned}
$$

Inverting these relations gives:

$$
\begin{aligned}
N_{\gamma} & =\frac{P_{c}^{\pi} \cdot N_{n c}-P_{n c}^{\pi} \cdot N_{c}}{P_{n c}^{\gamma}-P_{n c}^{\pi}} \\
N_{\pi} & =\frac{P_{n c}^{\gamma} \cdot N_{c}-P_{c}^{\gamma} \cdot N_{n c}}{P_{n c}^{\gamma}-P_{n c}^{\pi}}
\end{aligned}
$$

\section{Prompt Photon Fraction}

We have described the process by which the probability of photon conversion is used to determine the number of true prompt photons. Thus, the prompt photon fraction is defined as:

$$
f_{\gamma}=N_{\gamma} / N_{\gamma}^{o}
$$

and the fraction of QCD events as $f_{\pi}=1-f_{\gamma}$.

Table 8.6 shows the non-conversion probabilities for prompt photons and $\pi^{0}$, the 
extracted number of true prompt photons and $\pi^{0}$, and the prompt photon fraction. Figure 8.19 shows the distribution of $f_{\pi}$ in bins of $E_{T}$.

\begin{tabular}{|l|c|c|c|c|c|c|}
\hline$E_{T}$ bin $(\mathrm{GeV})$ & $P_{n c}^{\gamma}$ & $m_{\gamma}$ & $P_{n c}^{\pi}$ & $N_{\gamma}$ & $N_{\pi}$ & $f_{\gamma}$ \\
\hline \hline $25-39$ & 0.370 & 2.05 & 0.132 & 406.5 & 506.5 & $0.445 \pm 0.070$ \\
\hline $39-70$ & 0.363 & 2.14 & 0.118 & 389.9 & 418.1 & $0.483 \pm 0.073$ \\
\hline $70-200$ & 0.346 & 2.23 & 0.103 & 267.7 & 132.3 & $0.669 \pm 0.104$ \\
\hline
\end{tabular}

Table 8.6: Prompt photon fraction of the photon candidates.

The uncertainty on $f_{\pi}$ receives contributions from the statistical uncertainties on the observed number of converted and non-converted candidates, and systematic uncertainties on the CPR detector efficiency, the underlying event fluctuation rate, and the uncertainty on the photon conversion length. These contributions are shown in Table 8.7.

\begin{tabular}{|l|c|c|c|c|c|c|c|c|}
\hline$E_{T}$ bin $(\mathrm{GeV})$ & $f_{\pi}$ & $\delta N_{c}^{o}$ & $\delta N_{n c}^{o}$ & $\delta \epsilon_{C P R}$ & $\delta F_{U E}$ & $\delta X_{0}^{\gamma}$ & $\delta f_{\pi}$ & $\delta f_{\pi} / f_{\pi}$ \\
\hline \hline $25-39$ & 0.555 & 0.031 & 0.057 & 0.016 & 0.008 & 0.021 & 0.071 & 12.8 \\
\hline $39-70$ & 0.517 & 0.032 & 0.059 & 0.016 & 0.008 & 0.020 & 0.073 & 14.0 \\
\hline $70-200$ & 0.331 & 0.050 & 0.087 & 0.015 & 0.009 & 0.021 & 0.104 & 31.4 \\
\hline
\end{tabular}

Table 8.7: Uncertainty contributions on the QCD fraction $\left(f_{\pi}\right) . \delta f_{\pi} / f_{\pi}$ is given as a percentage.

\subsubsection{Corrected Jet $\rightarrow \gamma$ Fake Rate}

The corrected jet $\rightarrow \gamma$ fake rate $\left(r_{\gamma}\right)$ is defined as the probability for a QCD jet to pass the photon identification cuts, excluding prompt photons:

$$
\begin{aligned}
r_{\gamma} & =N_{\pi} / N_{j}=\frac{N_{\gamma}^{o}}{N_{j}} \frac{N_{\pi}}{N_{\gamma}^{o}} \\
& =r_{\text {raw }} \cdot f_{\pi}
\end{aligned}
$$




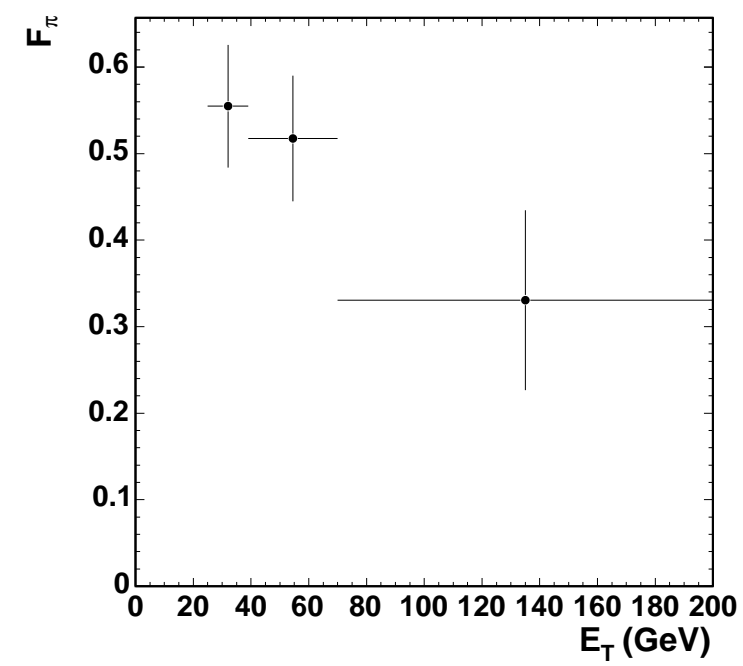

Figure 8.19: Fraction of fakes in the jet $\rightarrow$ photon fake rate sample, $f_{\pi}$.

Table 8.8 and Figure 8.20 show the corrected fake rate in bins of $E_{T}$. The uncertainty on $f_{\pi}$ dominates the uncertainty on $r_{\gamma}$.

\begin{tabular}{|l|c|c|}
\hline$E_{T}$ bin & $r_{\gamma} \times 1000$ & $\delta r_{\gamma} / r_{\gamma}(\%)$ \\
\hline \hline $25<E_{T}<39 \mathrm{GeV}$ & $0.47 \pm 0.06$ & 13.0 \\
\hline $39<E_{T}<70 \mathrm{GeV}$ & $0.36 \pm 0.05$ & 14.3 \\
\hline $70<E_{T}<200 \mathrm{GeV}$ & $0.20 \pm 0.06$ & 31.7 \\
\hline
\end{tabular}

Table 8.8: Corrected $j \rightarrow \gamma$ fake rate $\left(r_{\gamma}\right)$.

\section{Comparisons of Results to an Alternate CES Method}

The CES can be used as a discriminant between prompt photons and $\pi^{0}$, and a similar strategy to the one described here can be used to compute the raw fake rate, the prompt photon fraction and the corrected fake rate. The CES method works well at low $E_{T}$ where the photons from the $\pi^{0}$ are well separated [24]. This method is complementary to the CPR method. We compare the results from the 


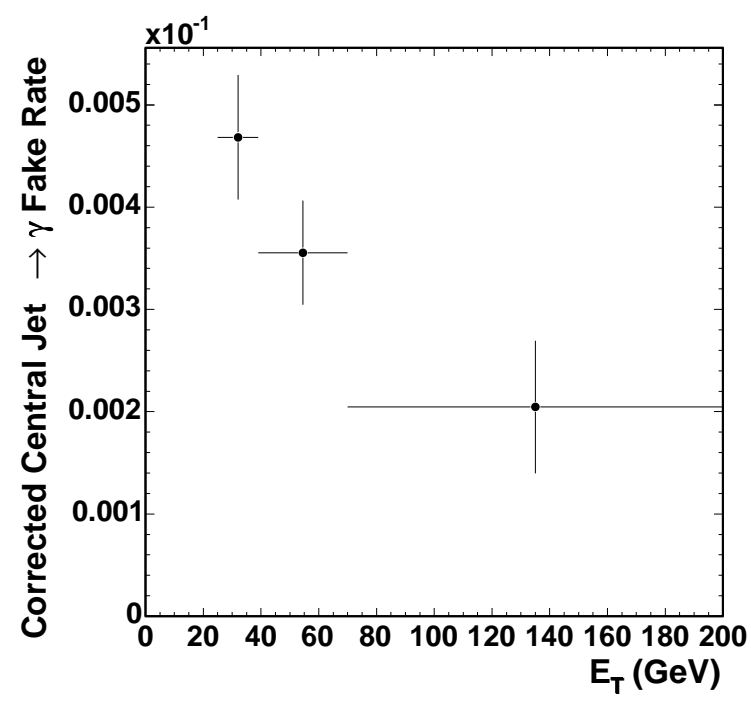

Figure 8.20: Corrected $j \rightarrow \gamma$ fake rate.

CPR method to the CES method using only Pre-January 2003 data and a slightly modified set of photon cuts [25]. We find good agreement between the two methods.

Figures 8.21 - 8.23 show the comparisons between the results obtained using the two methods. The discrepancy between the raw rates shown in Figure 8.21 is due to a difference in methods used to obtain the raw fake rate. The CES method required that all jets be well separated from one another $(\Delta R$ between all jets was $>0.8)$ in order to be included in the fake rate calculation. The CPR method did not have this requirement. Thus, it has a smaller raw fake rate since more non-isolated jets, incapable of faking a photon, are included in the denominator of $r_{\text {raw }}=N_{\gamma}^{o} / N_{j}$. This difference is then accounted for in the measurement of $f_{\pi}$ resulting in good agreement between the true fake rate for the two methods. 


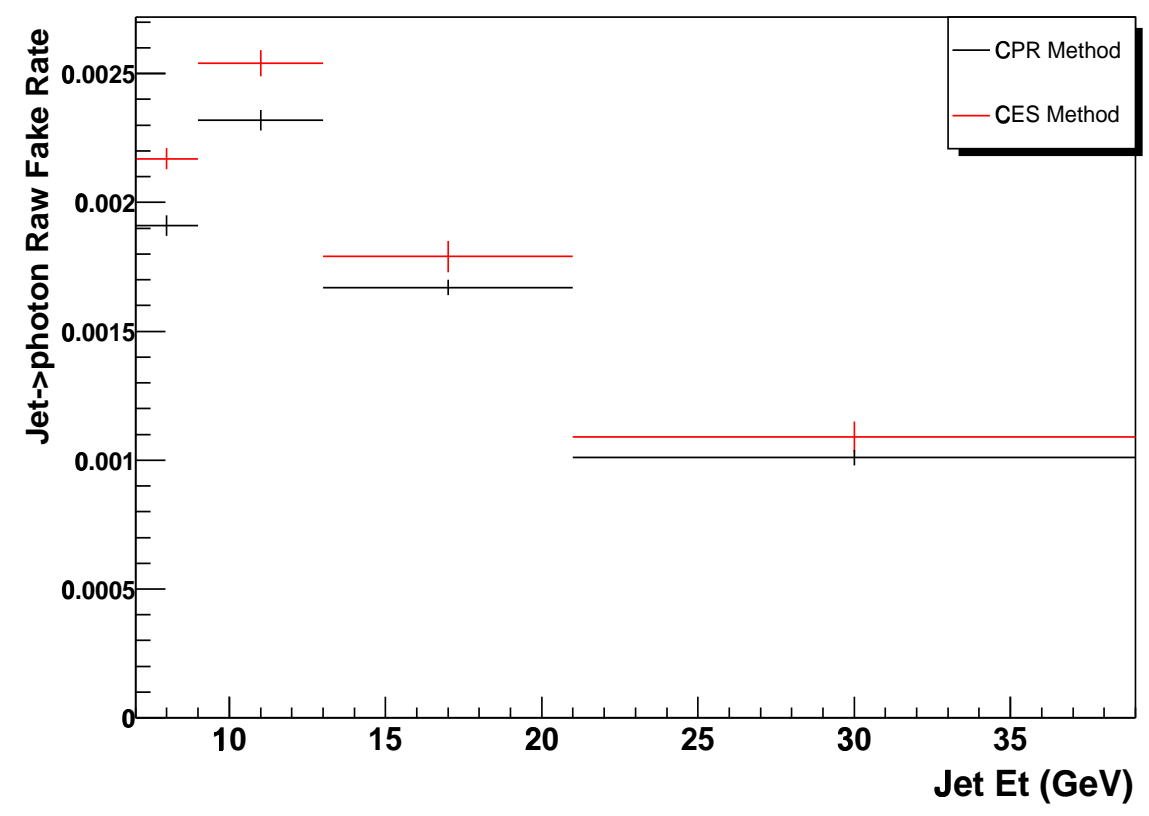

Figure 8.21: Comparison between the raw $j \rightarrow \gamma$ fake rate measured in this CPR-based analysis and the CES-based analysis [25].

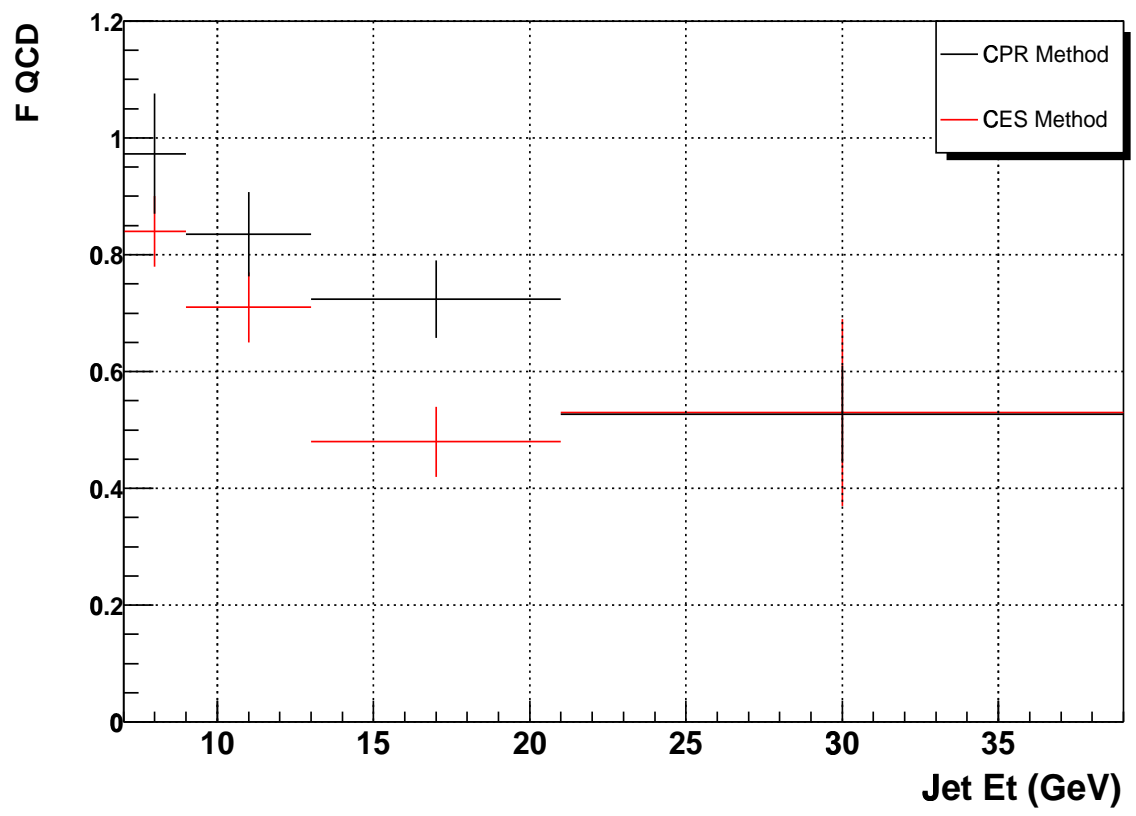

Figure 8.22: Comparison between the QCD fraction $f_{\pi}$ measured in this CPR based analysis and the CES based analysis [25]. 


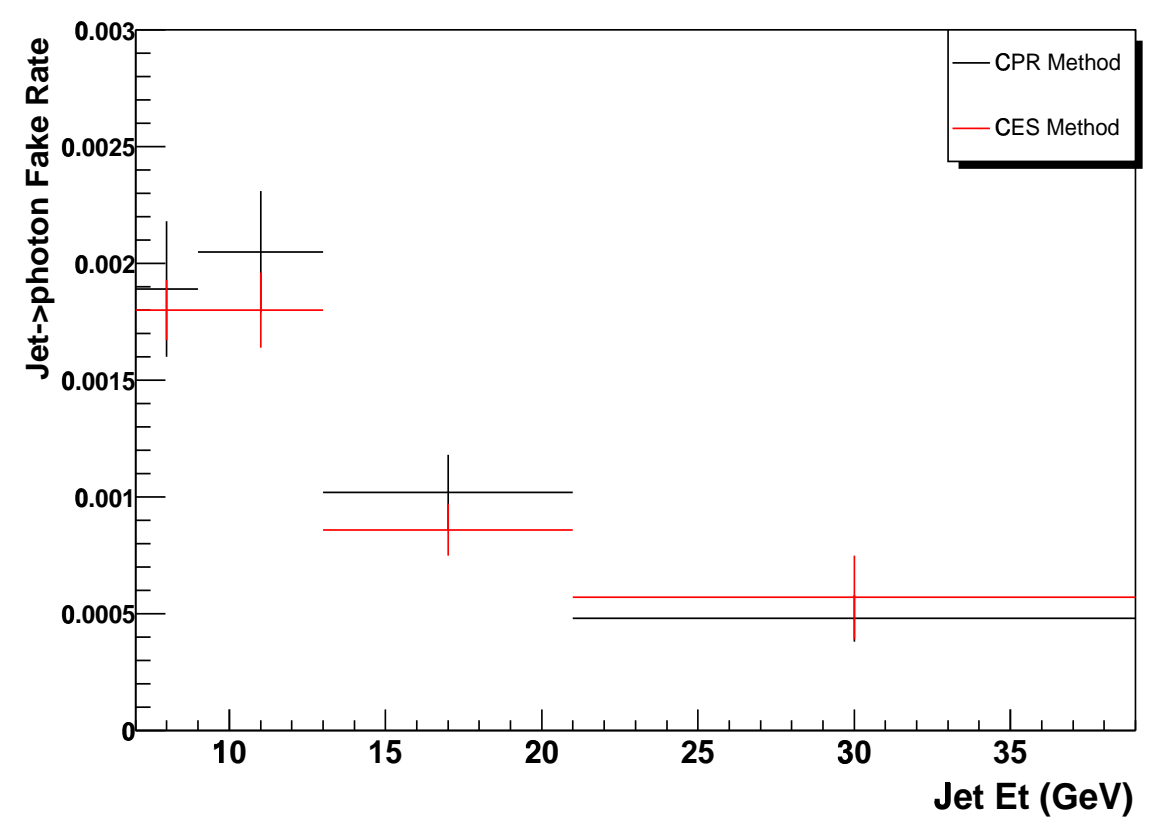

Figure 8.23: Comparison between the corrected $j \rightarrow \gamma$ fake rate measured in this CPR based analysis and the CES based analysis [25].

\subsubsection{Systematics}

The errors in Table 8.8 include the systematic error due to the error on the $f_{\pi}$ measurement. In addition, we study the uncertainty on $r_{\gamma}$ due to $\eta_{d}$ dependence and the difference in fake rate between the second-leading jet and the remaining jets in an event.

We measure the dependence of $r_{\gamma}$ on $\eta_{d}$ in Figure 8.24. The weighted average value of $r_{\gamma}$ is $\sim 0.00066 \pm 0.00001$. The standard deviation is 0.00034 , or $51 \%$ with respect to the mean.

In this analysis, we have considered all jets in the event except the leading $E_{T}$ jet, which we assume is the trigger jet. We now study the fake rate for the secondleading $E_{T}$ jet in the event (we refer to this jet as "Jet2") compared to the fake rate for remaining jets (we refer to these jets as "Jet345", regardless of how many jets 


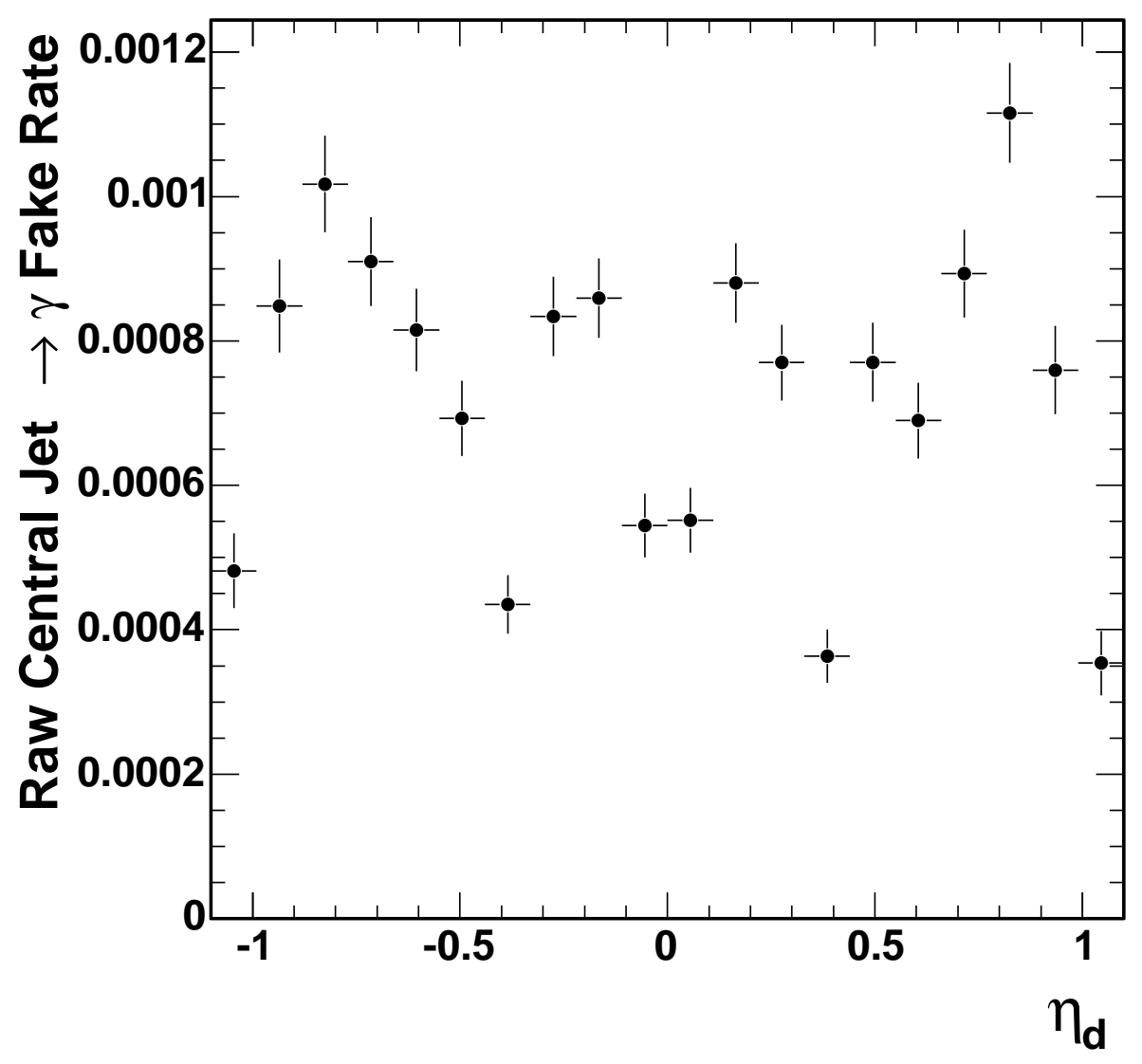

Figure 8.24: Raw $j \rightarrow \gamma$ fake rate measured as a function of detector $\eta$. 
are in the event). As shown in Figure 8.25, the raw fake rate for Jet2 is lower than for Jet345. The raw fake rate for Jet2 is around 0.0006, and for Jet345 it is around 0.0015 in the first $E_{T}$ bin.

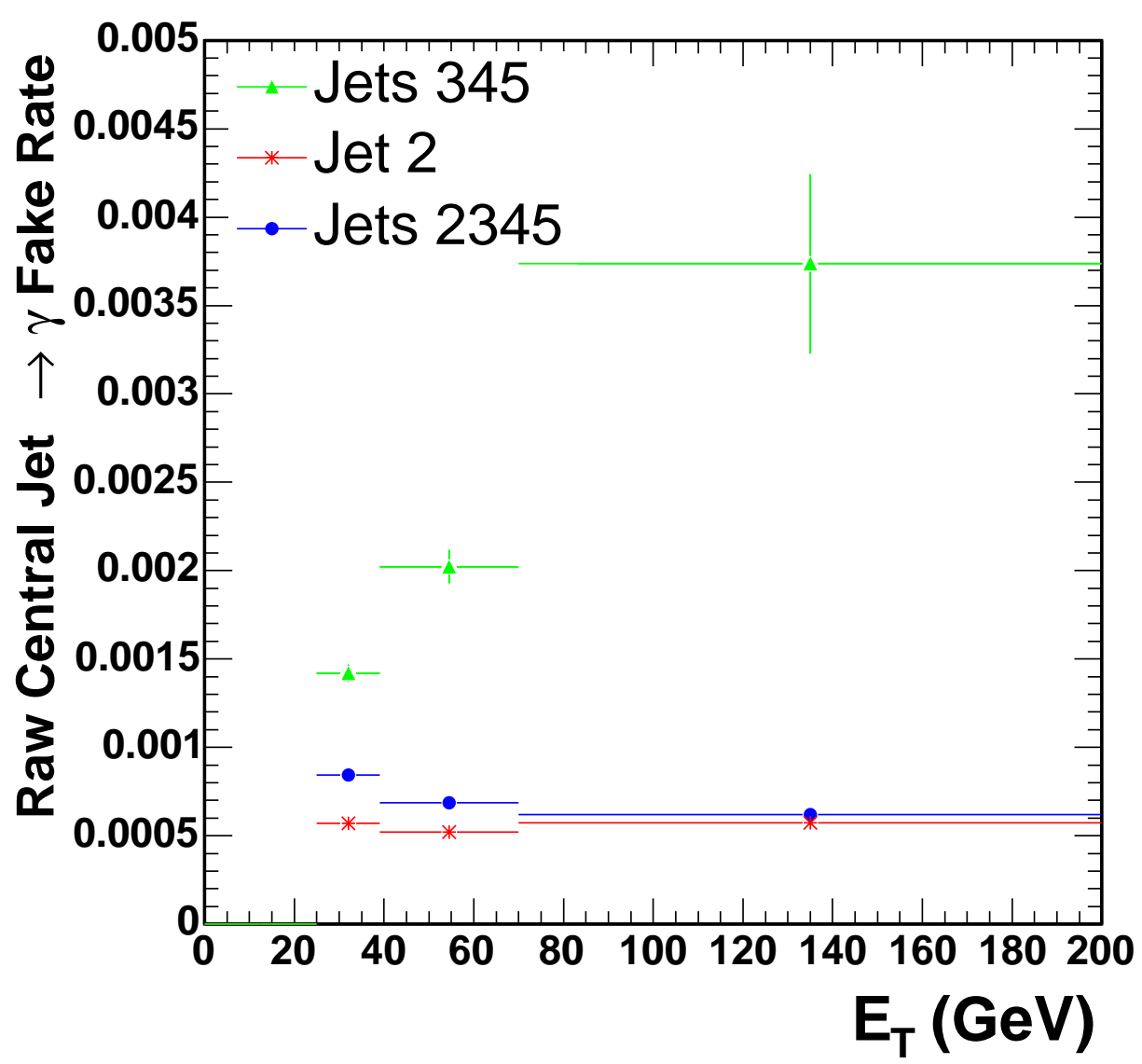

Figure 8.25: Raw $j \rightarrow \gamma$ fake rate measured in this CPR based analysis for jet 2, jets 345 and the combined fake rate.

Table 8.9 shows the Jet2 raw fake rate and $f_{\pi}$ in three $E_{T}$ bins. Table 8.10 shows the Jet345 raw fake rate and $f_{\pi}$ in bins of $E_{T}$. These tables clearly show that the raw fake rate for Jet2 is lower than for Jet345 in each $E_{T}$ bin. However, due in part to lack of statistics, we are unable to clearly see whether the $f_{\pi}$ for the two types of jets show similarly divergent behavior. 


\begin{tabular}{|l|c|c|c|c|c|}
\hline$E_{T}$ bin & $N_{j}$ & $N_{\gamma}^{o}$ & $r_{\text {raw }} \times 1000$ & $f_{\pi}$ & $r_{\gamma} \times 1000$ \\
\hline \hline $25<E_{T}<39 \mathrm{GeV}$ & 1253590 & 714 & $0.57 \pm 0.02$ & $0.644 \pm 0.098$ & $0.37 \pm 0.06$ \\
\hline $39<E_{T}<70 \mathrm{GeV}$ & 1815010 & 944 & $0.52 \pm 0.02$ & $0.510 \pm 0.085$ & $0.27 \pm 0.04$ \\
\hline $70<E_{T}<200 \mathrm{GeV}$ & 1020000 & 586 & $0.57 \pm 0.02$ & $0.330 \pm 0.109$ & $0.19 \pm 0.06$ \\
\hline
\end{tabular}

Table 8.9: Corrected $j \rightarrow \gamma$ fake rate $\left(r_{\gamma}\right)$.

\begin{tabular}{|l|c|c|c|c|c|}
\hline$E_{T}$ bin & $N_{j}$ & $N_{\gamma}^{o}$ & $r_{\text {raw }} \times 1000$ & $f_{\pi}$ & $r_{\gamma} \times 1000$ \\
\hline \hline $25<E_{T}<39 \mathrm{GeV}$ & 597856 & 849 & $1.42 \pm 0.05$ & $0.481 \pm 0.094$ & $0.68 \pm 0.13$ \\
\hline $39<E_{T}<70 \mathrm{GeV}$ & 227011 & 459 & $2.02 \pm 0.09$ & $0.535 \pm 0.124$ & $1.08 \pm 0.25$ \\
\hline $70<E_{T}<200 \mathrm{GeV}$ & 14454 & 54 & $3.74 \pm 0.51$ & $0.342 \pm 0.338$ & $1.28 \pm 1.26$ \\
\hline
\end{tabular}

Table 8.10: Raw $j \rightarrow \gamma$ fake rate, $f_{\pi}$, and corrected fake rate for Jet345 in the event.

Because the behavior of $f_{\pi}$ for Jet2 versus Jet345 is inconclusive, we use the $f_{\pi}$ measured using the combined results from both Jet2 and Jet345 (see Table 8.6) to determine the corrected fake rates for Jet2 and Jet345 separately. The corrected fake rates for Jet2 and Jet345 are shown in Table 8.11. The difference between these numbers and the combined corrected fake rates are used to quote asymmetric errors on the fake rate.

\begin{tabular}{|l|c|c|c|}
\hline$E_{T}$ bin & $\begin{array}{c}\text { Combined } \\
r_{\gamma} \times 1000\end{array}$ & $\begin{array}{c}\text { Jet } 2 \\
r_{\gamma} \times 1000\end{array}$ & $\begin{array}{c}\text { Jet345 } \\
r_{\gamma} \times 1000\end{array}$ \\
\hline \hline $25<E_{T}<39 \mathrm{GeV}$ & 0.47 & 0.32 & 0.79 \\
\hline $39<E_{T}<70 \mathrm{GeV}$ & 0.36 & 0.27 & 1.05 \\
\hline $70<E_{T}<200 \mathrm{GeV}$ & 0.20 & 0.19 & 1.24 \\
\hline
\end{tabular}

Table 8.11: Corrected $j \rightarrow \gamma$ fake rate $(r)$. We apply $f_{\pi}$ from the all jets measurement (jets $2,3,4,5)$ to the raw fake rates for Jet2 and Jet345 to obtain corrected fake rates.

The systematic error is determined by whichever of the following is greater: the uncertainty resulting from the $51 \%$ relative uncertainty due to $\eta_{d}$ fake rate 
dependence or the error due to the difference between the combined fake rate and the Jet2 (lower bound on error) or Jet345 (upper bound error). The resulting $r_{\gamma}$ and uncertainties is shown in Table 8.12.

\begin{tabular}{|l|c|}
\hline$E_{T}$ bin & $r_{\gamma} \times 1000$ \\
\hline \hline $25<E_{T}<39 \mathrm{GeV}$ & $0.47 \pm 0.06_{-0.24}^{+0.32}$ \\
\hline $39<E_{T}<70 \mathrm{GeV}$ & $0.36 \pm 0.05_{-0.18}^{+0.69}$ \\
\hline $70<E_{T}<200 \mathrm{GeV}$ & $0.20 \pm 0.06_{-0.10}^{+1.04}$ \\
\hline
\end{tabular}

Table 8.12: Corrected fake rate with asymmetric errors to account for the difference between the fake rate of Jet2 and Jet345 and the $\eta_{d}$ dependence, whichever is greater.

\subsubsection{Jet $E_{T} \rightarrow \gamma E_{T}$ Fragmentation Function}

The photon matched to the jet contains only a fraction of the jet energy. To apply $r_{\gamma}$, we need to know what photon energy corresponds to the jet energy. Figure 8.26 shows the profile distribution of $E_{T}^{\gamma} / E_{T}^{j}$ as a function of jet $E_{T}$ and $E^{\gamma} / E^{j}$ as a function of jet energy. The fragmentation of jet energy to photon energy is flat and about the same for both the $E_{T}$ and energy distributions. Thus, a jet's transverse energy is scaled by 0.944 to obtain the corresponding photon transverse energy. Figure 8.27 shows histograms of $E_{T}^{\gamma} / E_{T}^{j}$ fit to Gaussian distributions for a series of jet $E_{T}$ ranges. The plots show that 0.944 is a reasonable fragmentation value to use and that there is not much spread in the $E_{T}^{\gamma} / E_{T}^{j}$ distributions. 


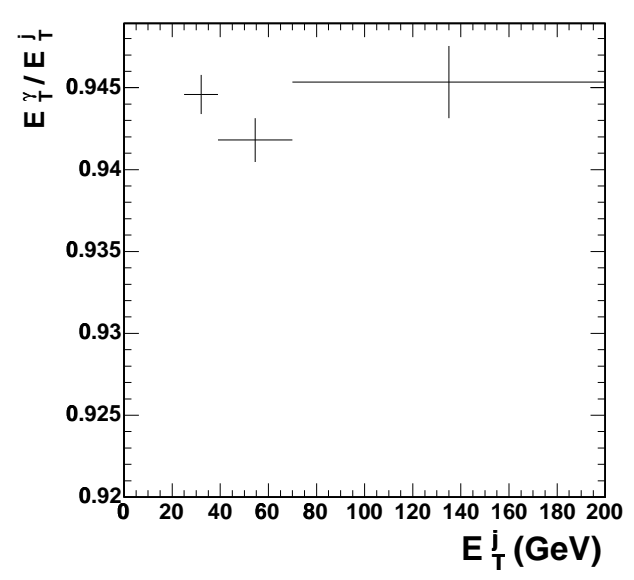

(a)

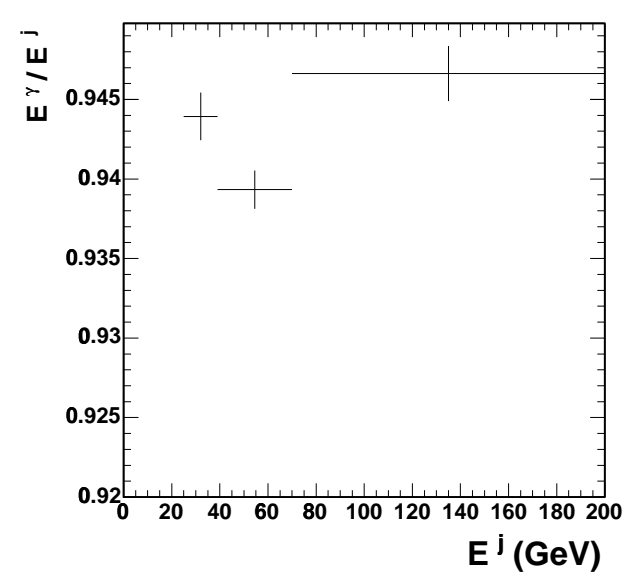

(b)

Figure 8.26: Figure (a) is a profile of the $E_{T}^{\gamma} / E_{T}^{j}$ as a function of jet $E_{T}$. Figure (b) is a profile of $E^{\gamma} / E^{j}$ as a function of jet energy.

\subsection{Unbiased Plug Jet $\rightarrow$ Electromagnetic Object Fake Rate}

The final fake rate to discuss is the unbiased plug jet $\rightarrow$ EM object fake rate for predicting background contributions in the plug region. This rate measures the probability that an unbiased jet is misidentified as a plug EM object.

\subsubsection{Data Samples}

As before, we obtain a sample of unbiased jets by using data triggered on the JET_20, JET_50, JET_70, and JET_100 triggers. The maximum $E_{T}$ jet is assumed to be the trigger jet, and to avoid any bias due to the jet trigger, we exclude this leading jet from the measurement. 


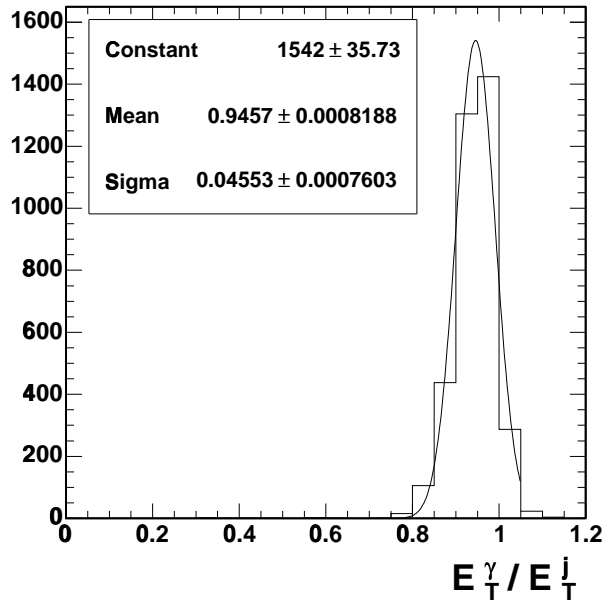

(a)

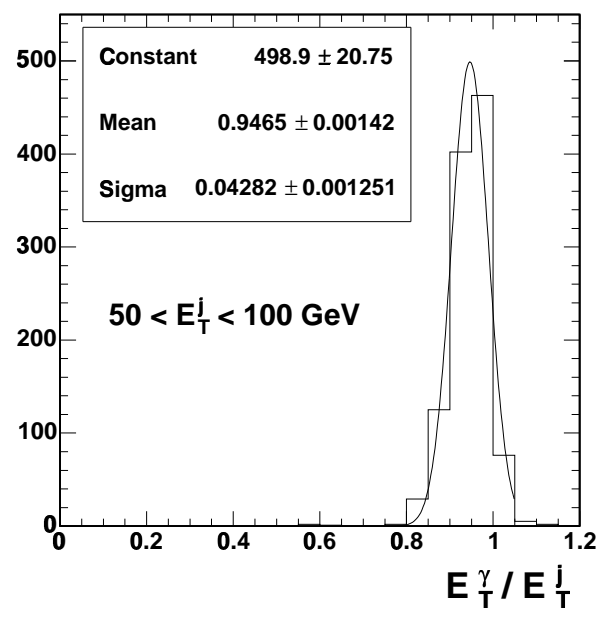

(c)

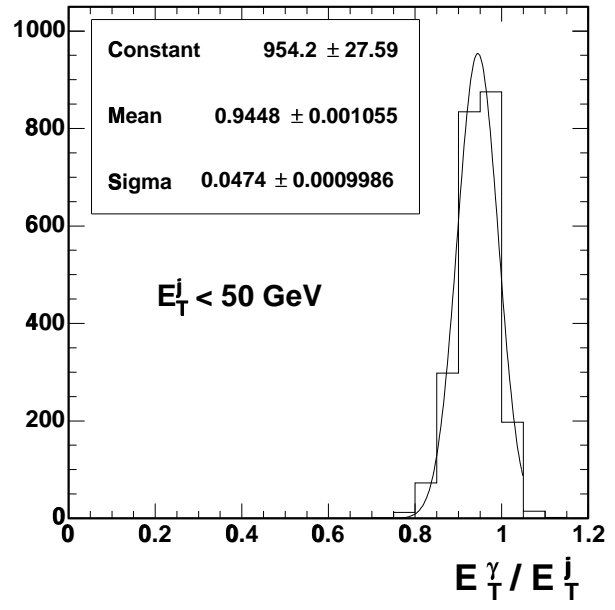

(b)

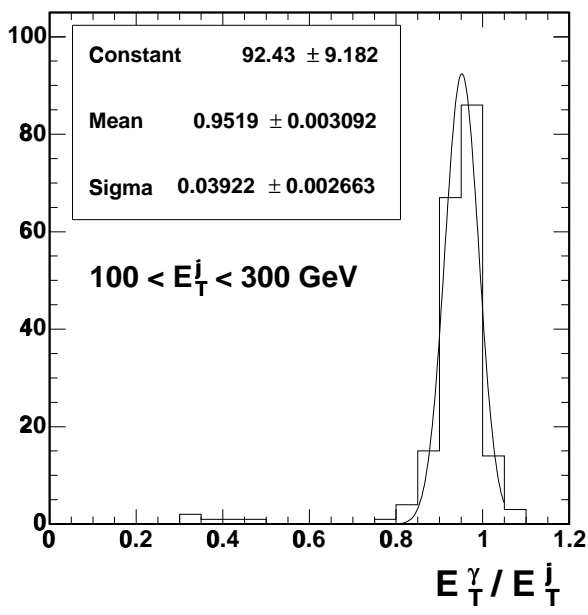

(d)

Figure 8.27: Distributions of $E_{T}^{\gamma} / E_{T}^{j}$ for different ranges of $E_{T}^{j}$. Figure (a) is for all $E_{T}^{j}$. Each is fit to a Gaussian. 


\subsubsection{Measurement}

The rate for an unbiased jet to fake a plug EM object $\left(r_{p}\right)$ is measured by selecting non-leading jets from the JET_20, JET_50, JET_70, and JET_100 samples, and counting the rate at which plug EM objects match the selected jets with $\Delta R<0.2$. The plug EM object fake rate, $r_{p}$, is defined as the rate at which unbiased jets are identified as plug EM objects. $r_{p}$ is given by $r_{p}=N_{p}^{o} / N_{j}$, where $N_{j}$ is the number of non-leading plug jets with $E_{T}>25 \mathrm{GeV}$ and $\left|Z_{v t x}\right|<60 \mathrm{~cm}$, and $N_{p}^{o}$ is the number of observed plug EM objects matched to these jets in a cone of 0.2 . The plug EM objects are selected using the selection criteria in Table 4.4. Figure 8.28 shows the separation of plug jets from plug EM objects in the events. Clearly, a $\Delta R<0.2$ cut is appropriate for matching plug jets to plug EM objects.

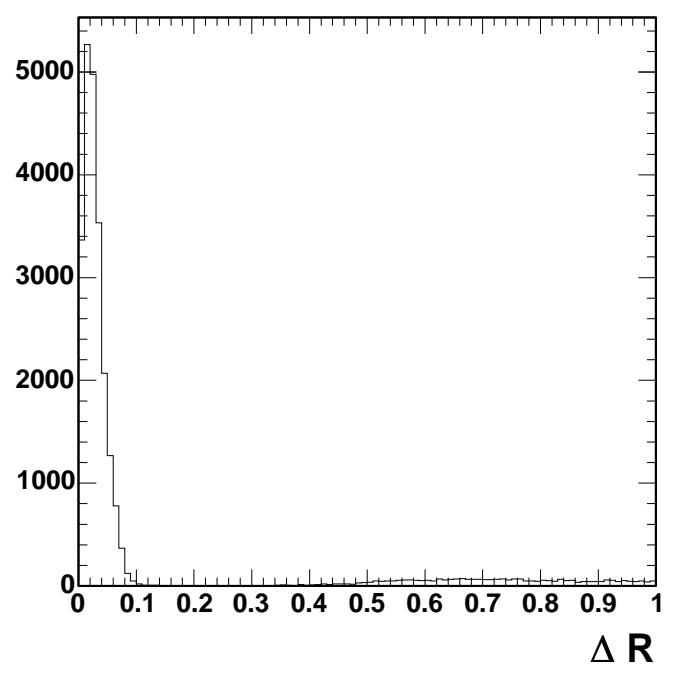

Figure 8.28: $\Delta R$ between jets and and plug EM object candidates. We use a cut of $\Delta R<0.2$ to match jets to plug EM objects.

The fake rate as a function of detector $\eta$ is shown in Figure 8.29. Because the fake rate strongly depends on $\eta_{d}$, we study fake rate dependence on $E_{T}$ in three different $\eta_{d}$ regions: $1.2<\left|\eta_{d}\right|<1.7,1.7<\left|\eta_{d}\right|<2.3$, and $2.3<\left|\eta_{d}\right|<2.8$. The 
fake rates for these three $\eta_{d}$ regions as a function of $E_{T}$ are shown in Figure 8.30.

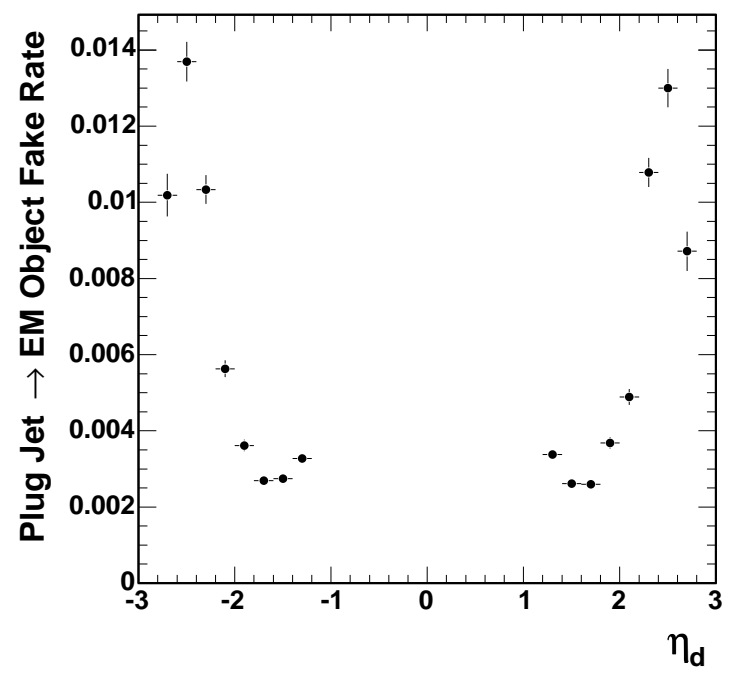

Figure 8.29: Plug jet $\rightarrow$ EM object fake rate as a function of detector $\eta$.

We fit the fake rate as a function of $E_{T}$ for each of the three $\eta_{d}$ regions. The $\eta_{d}$ region $1.2<\left|\eta_{d}\right|<1.7$ can be fit by a quadratic function, while the $\eta_{d}$ regions $1.7<\left|\eta_{d}\right|<2.3$ and $2.3<\left|\eta_{d}\right|<2.8$ can be fit with a simple linear fit. The fits for each region are shown in Figures 8.31 - 8.33. We use the fit results to determine the central value of the plug jet $\rightarrow$ plug EM object fake rate.

\subsubsection{Systematics}

We compare the fake rate for the second highest transverse energy jet (Jet2) to that for the remaining jets (Jet345). These results are shown in Figures 8.31 - 8.33. The central value of the fake rate is obtained from the Jet2 - Jet345 combined fake rate result. Asymmetric systematic errors due to Jet2 - Jet345 differences are applied.

The Jet2 fake rate is very similar to the central value, particularly at high $E_{T}$. The difference between the Jet2 fake rate and the combined fake rate is folded 


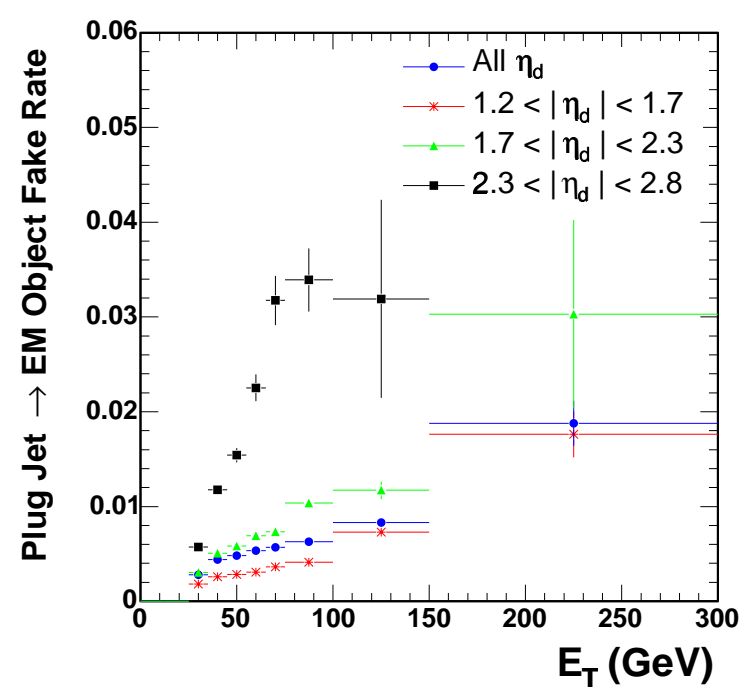

Figure 8.30: Plug jet $\rightarrow$ EM object fake rate as a function of $E_{T}$ for all $\eta$ regions as well as for the $1.2<\left|\eta_{d}\right|<1.7,1.7<\left|\eta_{d}\right|<2.3$, and $2.3<\left|\eta_{d}\right|<2.8$ regions separately.

into the systematic error by considering the largest difference in each $\eta_{d}$ bin. For $1.2<\left|\eta_{d}\right|<1.7$, Jet2 differs by at most $15 \%$ of the central value; for $1.7<\left|\eta_{d}\right|<2.3$, Jet2 differs at most by $15 \%$ of the central value; and for $2.3<\left|\eta_{d}\right|<2.8$, the Jet2 fake rate differs by at most $10 \%$. We use these values in calculating the lower bound on $r_{p}$.

The Jet345 fake rate differs more significantly from the central value than does the fake rate for Jet2 jets. The difference between the central value and Jet345 is accounted for by fitting the Jet345 distribution as shown and using the difference from the central value fit as an upper bound on $r_{p}$ due to systematic error.

\subsubsection{Jet $E_{T} \rightarrow$ Plug EM Object $E_{T}$ Fragmentation Function}

The plug EM object associated with a jet contains a fraction of the jet energy. This is because the energy of the plug EM cluster is restricted to the towers in the cluster 


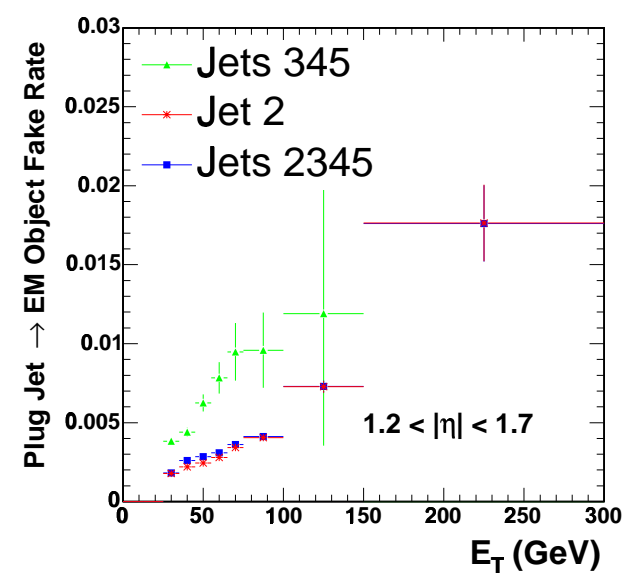

(a)

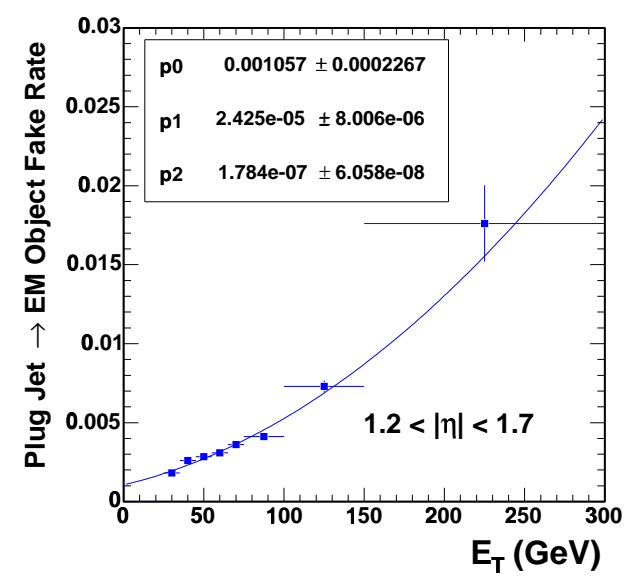

(b)

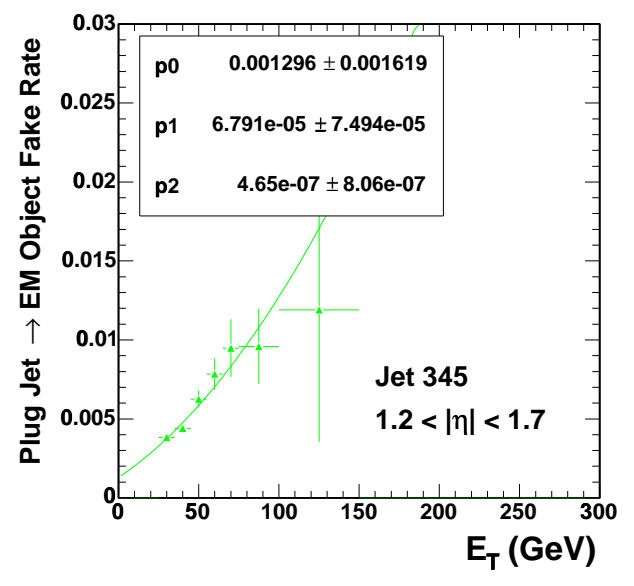

(c)

Figure 8.31: Fake rate distributions for $1.2<\left|\eta_{d}\right|<1.7$. Figure (a) shows the distribution for all jets and for Jet2 and Jet345. Figure (b) shows the fit for all jets, Figure (c) shows the fit for Jet345 jets. 


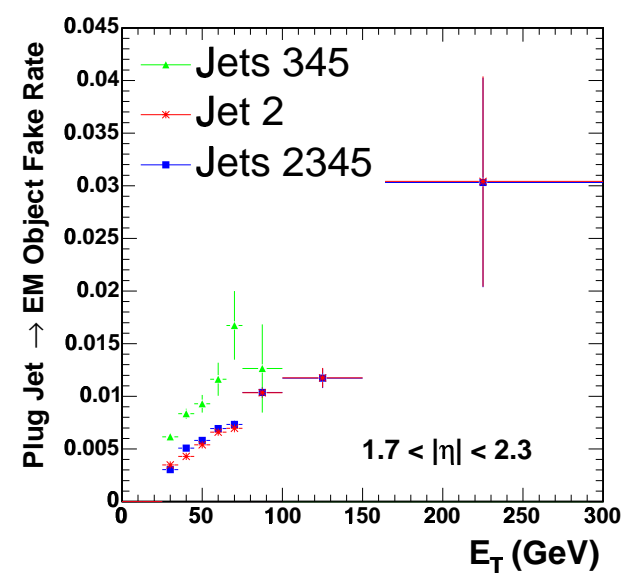

(a)

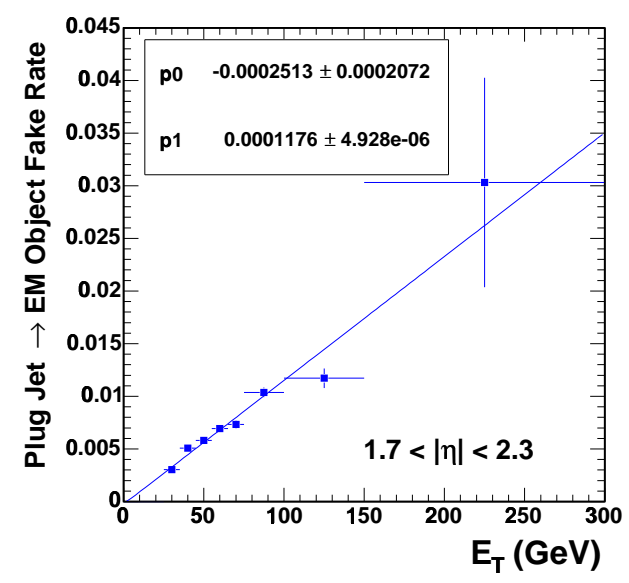

(b)

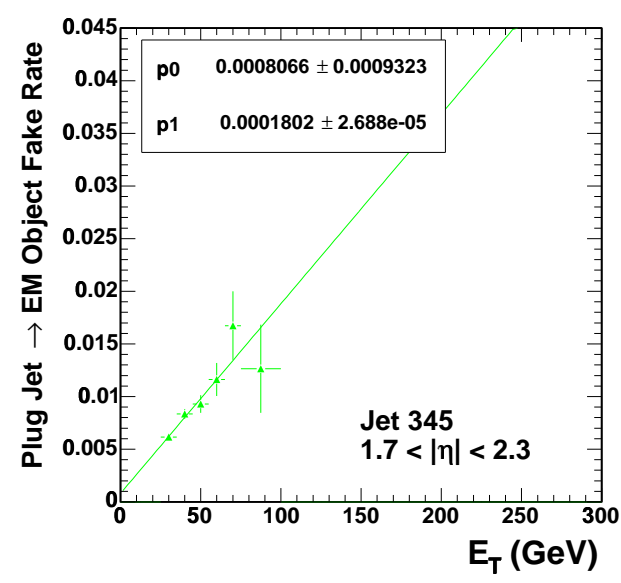

(c)

Figure 8.32: Fake rate distributions for $1.7<\left|\eta_{d}\right|<2.3$. Figure (a) shows the distribution for all jets and for Jet2 and Jet345. Figure (b) shows the fit for all jets, Figure (c) shows the fit for Jet345 jets. 


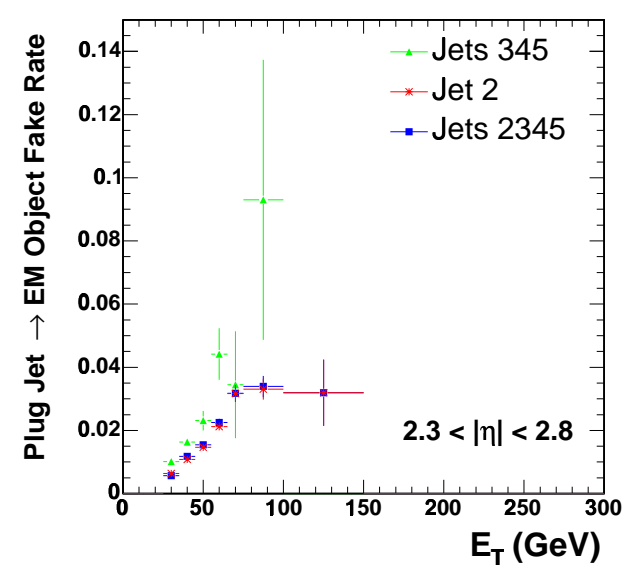

(a)

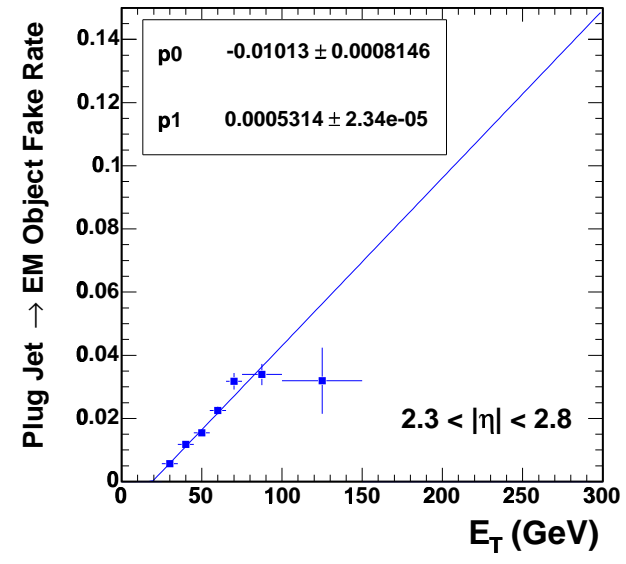

(b)

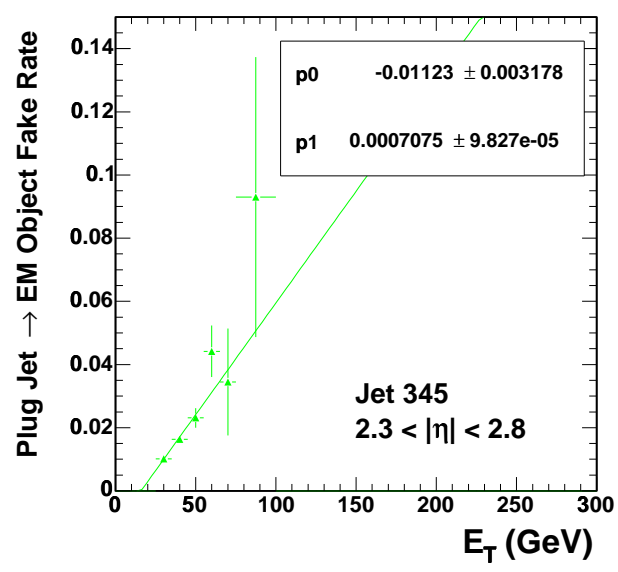

(c)

Figure 8.33: Fake rate distributions for $2.3<\left|\eta_{d}\right|<2.8$. Figure (a) shows the distribution for all jets and for Jet2 and Jet345. Figure (b) shows the fit for all jets, Figure (c) shows the fit for Jet345 jets. 
and the jet energy includes all the energy in a cone of $R=0.4$.

Figure 8.34 shows the fragmentation function of jet energy to plug EM object energy. We use the distribution in Figure 8.34(a) to determine the transverse energy of the plug EM object faked by the jet. Figure 8.35 demonstrates that there is not much spread in the jet $E_{T} \rightarrow$ plug EM object $E_{T}$ ratio values and so the fit of Figure $8.34(\mathrm{a})$ is reasonable.

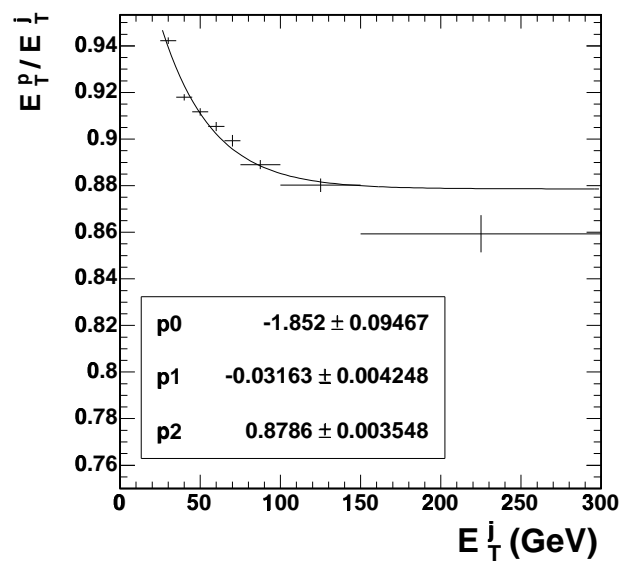

(a)

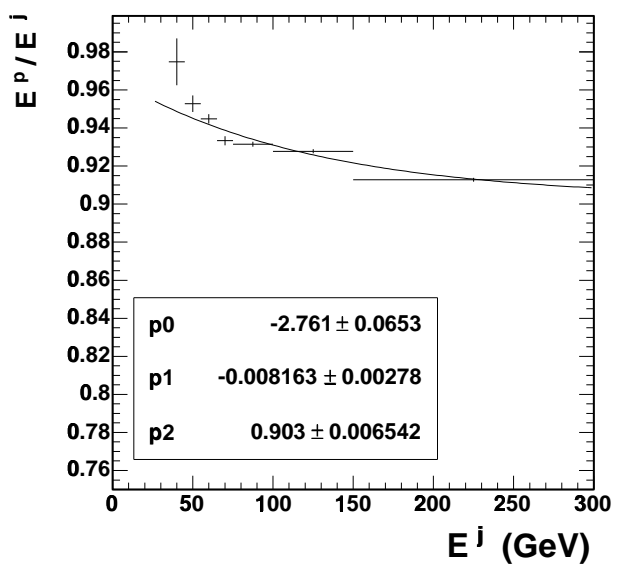

(b)

Figure 8.34: Figure (a) is a profile of the $E_{T}^{p e m} / E_{T}^{j}$ as a function of jet $E_{T}$. Figure (b) is a profile of $E^{p e m} / E^{j}$ as a function of jet energy. 


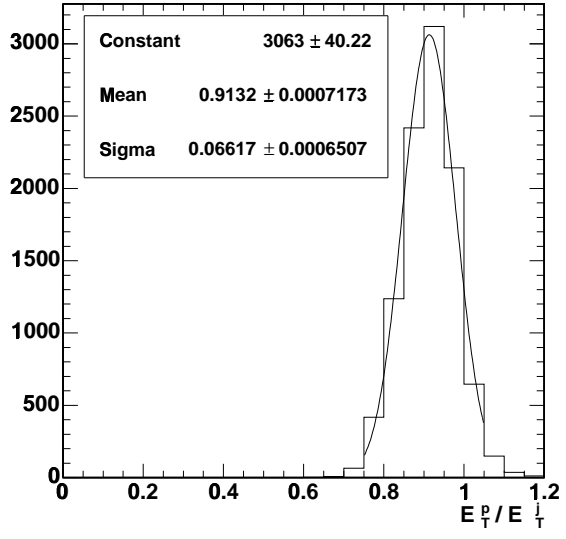

(a)

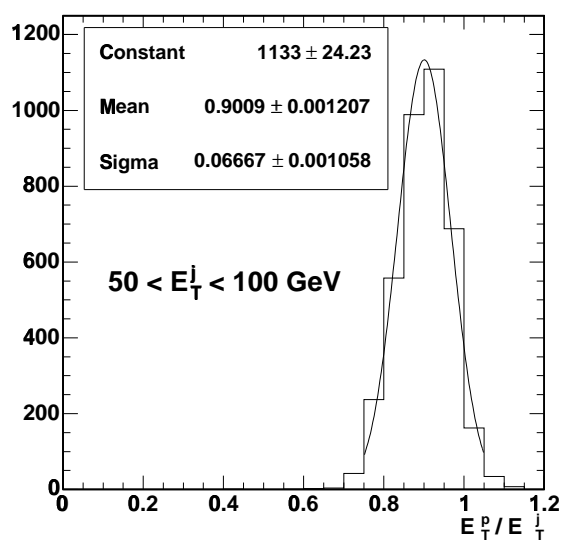

(c)

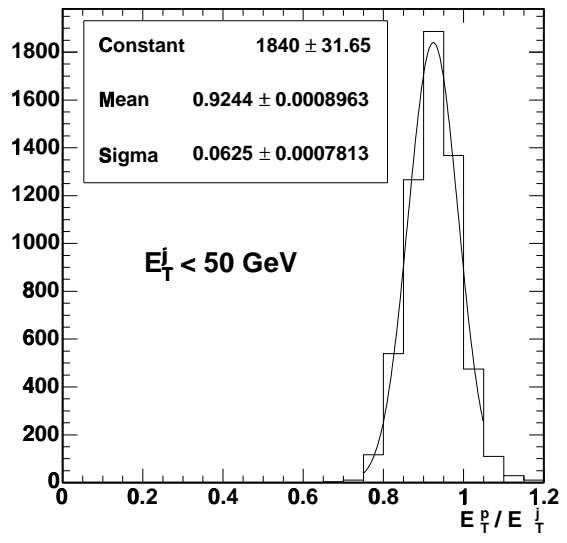

(b)

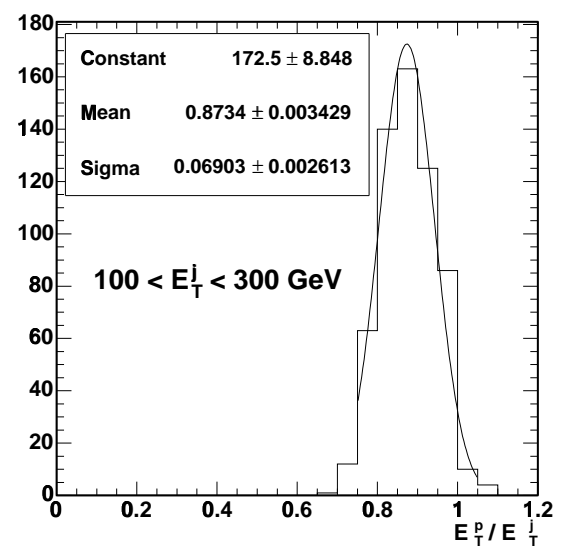

(d)

Figure 8.35: Distributions of $E_{T}^{p e m} / E_{T}^{j}$ for different ranges of $E_{T}^{j}$. Figure (a) is for all $E_{T}^{j}$. Each is fit to a Gaussian. 


\section{Chapter 9}

\section{Backgrounds}

There are both standard model processes and misidentification sources that contribute to dielectron + photon background. The primary background contribution is purely standard model $Z+\gamma$ production. The standard model background contributions and the measurements of backgrounds due to misidentification sources are discussed in this chapter.

Backgrounds from misidentification sources include $Z(\rightarrow e e)+$ jet production where a jet fakes a photon, multijet background where two jets fake electrons and one jet fakes a photon, diphoton+jet events where a jet fakes an electron, and $W(\rightarrow e \nu)+$ jets where jets fake an electron and a photon.

Additionally, standard model $W(\rightarrow e \nu) Z(\rightarrow e e)$ and $A(\rightarrow e e) Z(\rightarrow e e)$ productions are sources of background when one of the electrons is misidentified as a photon. This background affects the plug region, where there are no tracking requirements, much more than the central region.

A final background that is considered is standard model $t \bar{t}$ production:

$$
q+\bar{q} \rightarrow t\left(\rightarrow W^{+} b \rightarrow e^{+} \nu_{e} b\right)+\bar{t}\left(\rightarrow W^{-} b \rightarrow e^{-} \bar{\nu}_{e} b\right)
$$

where one of the $b$ quarks radiates a hard photon. 


\section{$9.1 Z / \gamma^{*}+\gamma$}

The standard model predicts $Z+\gamma$ and Drell - Yan $+\gamma$ production. We refer to this background as $Z \gamma$, where Drell-Yan inclusion is implied. When the $Z$ boson decays leptonically to two electrons, the final state is two electrons and a photon, exactly the signature of this search. $Z \gamma$ production is the largest source of background in this analysis.

This background is studied using Uli Baur and E.L. Berger's leading order ZGAMMA Monte Carlo matrix element generator [26]. The tree-level Monte Carlo calculation takes into consideration $Z \gamma$ production from both initial state radiation (radiation of a photon from an incoming quark) and radiative $\mathrm{Z}$ decays (where a photon is produced due to final state radiation from the lepton, also called inner bremsstrahlung).

The output of the ZGAMMA Monte Carlo generator is run through CdfSim and full data reconstruction. We impose the data selection criteria for electrons and photons and study the $M_{e e}, M_{e \gamma}$, and $M_{e e \gamma}$ distributions of the surviving events. In order to use the Monte Carlo distributions to predict the expected $Z \gamma$ signal in data, the Monte Carlo luminosity $\left(\mathcal{L}_{M C}\right)$ must be normalized to the data luminosity. The Monte Carlo luminosity is defined by:

$$
\mathcal{L}_{M C}=\frac{N_{\text {gen }}}{\sigma_{M C} \cdot K_{f}}
$$

where $N_{g e n}$ is the number of generated Monte Carlo events and $\sigma_{M C}$ is the generated cross-section at leading order. $K_{f}$ is the K-factor which is the NLO QCD correction factor. To normalize the Monte Carlo to the data, each event that passes the 
dielectron + photon criteria is weighted by:

$$
W=\frac{\mathcal{L}_{\text {data }}}{\mathcal{L}_{M C}}
$$

where $\mathcal{L}_{\text {data }}$ is the data luminosity.

The kinematic requirements at generator level for this sample yield a production $\sigma_{M C}=0.451 \mathrm{pb} . K_{f}$ is equal to 1.33 .

In addition to normalizing the simulation to the data, we must also make corrections based on differences observed between the simulation and the data. The kinematics of $Z \gamma$ events are more similar to $Z \rightarrow e e$ events than $\mathrm{e}^{*}$ events. Thus, we use the plug efficiency scale factor of 0.953, as explained in Section 6.1.4. The error on the plug efficiency scale factor is 0.024 , which is half the difference between 0.953 and 1. We apply the trigger efficiency of $0.9621 \pm 0.0006$ to events with only one central electron. To account for the difference between data and simulation of the efficiency of the $Z_{v t x}<60 \mathrm{~cm}$ requirement, there is a weight factor of $0.993 \pm 0.005$ applied.

\subsection{1 $Z_{\gamma}$ Background Prediction}

The expected number of $Z \gamma$ events integrated over all masses is $2.58 \pm 0.08$ (stat)

events: $1.05 \pm 0.05($ stat $)$ central events and $1.54 \pm 0.06$ (stat) plug events. The integrated number of e $\gamma$ entries is $5.56 \pm 0.11$ (stat) events, where $2.09 \pm 0.07$ (stat) are from central events and $3.47 \pm 0.09$ (stat) are from plug events. 


\subsection{2 $\quad Z_{\gamma}$ Background Systematics}

The primary sources of error on the $Z \gamma$ background estimation are due to the data luminosity measurement (6\%), the K-factor (5\%), and the cross-section uncertainty due to PDFs (5\%). Additional sources are from statistics, PDF uncertainty on acceptance, extra material in the simulation, electron, photon and plug EM object ID efficiencies, trigger efficiency, $Z_{v t x}$ efficiency, energy scale, and energy resolution.

Ideally, the error contributions from these sources would be measured as a function of $M_{e \gamma}$ and $M_{e e \gamma}$. However, we are statistically limited and must use the errors on the integrated number $M_{e e \gamma}$ and $M_{e \gamma}$ entries as the error for all mass values. This results in an underestimation of the error at low mass and overestimation at high mass. It is the high mass region that is of interest. The systematic errors are measured separately for the predicted number of $Z \gamma$ events $\left(M_{e e \gamma}\right)$ and the integrated number of $M_{e \gamma}$ entries.

\section{Parton Distribution Function Uncertainty on Acceptance}

The study of the PDF uncertainty on the $\mathrm{e}^{*}$ total signal acceptance at different mass values in Section 7.3.2 indicates that the PDF uncertainty is independent of the hard interaction. Thus, we use $1.04 \%$, the PDF uncertainty on the total signal acceptance for $e^{*}$ production, as the relative error on $Z \gamma$ background due to PDFs.

\section{Parton Distribution Function Uncertainty on Cross-section}

The uncertainty on the $Z / \gamma^{*}+\gamma$ production cross-section due to uncertainties in the PDFs is described in [26]. The production cross-sections resulting from PDF functions MRST 72-76 were compared to the standard, CTEQ5L. An error of 5\% on the cross-section is used due to the parton distribution functions. 


\section{Extra Material in the Simulation}

The default simulation of the CDF detector does not accurately describe the amount of material in the physical detector. This is accounted for by adding "phantom" layers of material to the simulation. See Section 7.3.7 for further discussion of the extra material. The relative error due to the extra material in the simulation is $3.9 \%$ on the integrated number of expected $Z \gamma$ background events. The relative error on the number of expected $M_{e \gamma}$ entries is $3.8 \%$.

\section{Central Electron Identification Efficiency}

The measurement of central electron identification efficiency in data and simulation is described in Section 6.1.1. The difference between the electron ID efficiency measured in data and simulation is $1 \%$. We apply a weight factor to electron objects of $1 \pm 0.01$ to determine the error on the $Z \gamma$ background due to electron ID efficiency uncertainty. The relative error on the total number of $Z \gamma$ events is $1.6 \%$. A relative error of $1.6 \%$ is also applicable to the $M_{e \gamma}$ distribution.

\section{Central Photon Identification Efficiency}

The "emulated" central photon identification efficiency is measured using $Z$ electrons in data and compared to simulation, as described in Section 6.1.2. There is a $3 \%$ difference between the data and simulation. So, the weight given to central photons is fluctuated up and down by $3 \%$, where the central value of the weight is 1. The relative error on the integrated number of expected $Z \gamma$ events is $2.0 \%$. The relative error on the integrated number of $M_{e \gamma}$ entries is $1.8 \%$. 


\section{Plug EM Object Identification Efficiency}

The plug EM object identification efficiency is discussed in Section 6.1.3. We found that the detector simulation overestimated the efficiency of electrons with kinematics similar to those from $Z \rightarrow e e$ events. This was due to mis-modeling of energy in the plug region of the detector. To account for this, a 0.953 efficiency weight factor is applied to plug objects and fluctuated it by its error, 0.024, to determine the error. There is a relative error of $1.9 \%$ on the total number of expected $Z \gamma$ candidates. For the integrated number of $e \gamma$ combinations, there is a $2.1 \%$ relative error due to plug EM object ID efficiency uncertainty.

\section{Trigger Efficiency}

As discussed in Section 6.2, the trigger efficiency of the ELECTRON_CENTRAL_18 trigger path is $(96.21 \pm 0.06($ stat $)) \%$. A weight of 0.9621 if applied to events with only one central electron. Events with two central electrons have two chances to fire the trigger, and, for those events, the trigger is fully efficient. We fluctuate the weight factor by the trigger error, 0.0006, and find the relative error due to trigger efficiency is $0.04 \%$ for the integrated number of $Z \gamma$ events and $0.05 \%$ for the integrated number of $M_{e \gamma}$ entries.

\section{$Z_{v t x}$ Efficiency}

The $Z_{v t x}$ finding efficiency is discussed in Section 6.3. The relative error due to the $Z_{v t x}$ efficiency uncertainty is $0.5 \%$. 


\section{Energy Scale}

The details of the energy scales and resolutions are in Chapter 5. Here we discuss the uncertainty on the $Z \gamma$ background due to errors on energy scale.

The error due to the central energy scale is measured by weighting the energy of central objects by $(1 \pm 0.001)$. The relative error on the total number of $Z \gamma$ events is measured to be $0.35 \%$. The relative error on the number of $M_{e \gamma}$ entries is $0.36 \%$.

The error due to the plug energy scale is determined by scaling the energy of plug objects by $(1 \pm 0.002)$. The relative error on the number of $Z \gamma$ events due to the west plug energy scale is $0.25 \%$. The relative error on the number of $M_{e \gamma}$ entries due to the west plug energy scale is $0.23 \%$. The relative error on the number of $Z \gamma$ events due to the east plug energy scale is $0.41 \%$. The relative error on the number of $M_{e \gamma}$ entries due to the east plug energy scale is $0.49 \%$.

We assume the energy scale errors in the west and east plug are uncorrelated. The energy scale in the central detector is correlated with the energy scale in the plug. Thus, the errors due to west and east plug scaling are added in quadrature and the plug and central scale errors are added linearly. The result is a $0.83 \%$ relative error on the integrated number of $Z \gamma$ events and $0.90 \%$ relative error on the number of $M_{e \gamma}$ entries.

\section{Energy Resolution}

Error due to energy resolution in the central calorimeter is determined by fluctuating the central energy smearing correction, 0.0317, by its error, 0.0023. There is a relative error of $0.37 \%$ on the integrated number of $Z \gamma$ events and a relative error of $0.31 \%$ on the integrated number of $M_{e \gamma}$ entries.

The west plug energy resolution contribution to the error is measured by fluc- 
tuating the west plug energy smearing correction, 0.019 , by its error, 0.0065 . The relative error on the number of predicted $Z \gamma$ events is $0.08 \%$. The relative error on the integrated number of $M_{e \gamma}$ entries is $0.11 \%$.

The east plug energy resolution contribution to the error is measured by fluctuating the east plug energy smearing correction, 0.019 , by its error, 0.0080 . The relative error on the number of predicted $Z \gamma$ events is $0.24 \%$. The relative error on the integrated number of $M_{e \gamma}$ entries is $0.30 \%$.

The energy resolution errors in the west and east plug calorimeters are assumed to be uncorrelated. The energy smearing correction in the central calorimeter is correlated with the smearing correction applied in the plug calorimeters. We add the errors due to west and east plug smearing corrections in quadrature. The plug error is then added linearly to the central error. There is a relative error of $0.62 \%$ on both the predicted number of $Z \gamma$ events and the number of $M_{e \gamma}$ entries due to energy resolution uncertainty.

\subsubsection{Summary}

The systematic uncertainties on the total number of $Z \gamma$ events are summarized in Table 9.1. The total relative systematic error is $10.67 \%$. Thus, the predicted number of $Z \gamma$ events is $2.58 \pm 0.08($ stat $) \pm 0.28($ sys) events.

The systematic error contributions on the total number of $M_{e \gamma}$ entries are summarized in 9.1. The total relative systematic error is $10.64 \%$. Thus, the predicted number of $M_{e \gamma}$ entries is $5.56 \pm 0.11($ stat $) \pm 0.59($ sys $)$. 


\begin{tabular}{|l|c|c|}
\hline $\begin{array}{l}\text { Error } \\
\text { Source }\end{array}$ & $\begin{array}{c}\text { Rel Err (\%) } \\
M_{e e \gamma}\end{array}$ & $\begin{array}{c}\text { Rel Err (\%) } \\
M_{e \gamma}\end{array}$ \\
\hline \hline Luminosity & 6.0 & 6.0 \\
\hline \hline K-factor & 5.0 & 5.0 \\
\hline PDF Cross-section & 5.0 & 5.0 \\
\hline PDF Acceptance & 1.04 & 1.04 \\
\hline \hline Extra Material & 3.9 & 3.8 \\
\hline Electron ID & 1.6 & 1.6 \\
\hline Photon ID & 2.0 & 1.8 \\
\hline Plug Object ID & 1.9 & 2.1 \\
\hline Trigger Efficiency & 0.04 & 0.05 \\
\hline$Z_{v t x}$ Efficiency & 0.5 & 0.5 \\
\hline Energy Scale & 0.83 & 0.90 \\
\hline Energy Resolution & 0.62 & 0.62 \\
\hline \hline Total & 10.67 & 10.64 \\
\hline
\end{tabular}

Table 9.1: Summary of systematic error contributions for the $Z \gamma$ background prediction.

\section{2 $Z(\rightarrow e e)+$ jet Background}

The background prediction due to $Z+$ jet events is the second largest background in this analysis. The $Z+$ jet background contribution is measured by applying the appropriate fake rate to $Z+$ jet events in the signal data set.

\subsubsection{Central Region Measurement}

The central region search requires two central electrons accompanied by an additional central jet. Each entry in the ee, ee $\gamma$, and e $\gamma$ invariant mass plots is weighted by the rate for an unbiased central jet to fake a central photon evaluated at the jet $E_{T}$. The expected photon $E_{T}$ that is used in the invariant mass calculations is computed from the jet $E_{T} \rightarrow \gamma E_{T}$ fragmentation function that was described in Section 8.3.5. 


\subsubsection{Plug Region Measurement}

There are three possible scenarios for $Z+$ jet background in the plug search, each of which requires one central electron. Each entry in the ee, ee $\gamma$, and e $\gamma$ invariant mass plots is weighted according to the type of event. The first possibility involves two central electrons accompanied by a plug jet. In this case, the event is weighted by the plug fake rate evaluated at the $E_{T}$ and $\eta$ of the jet. We apply the jet $E_{T} \rightarrow$ plug EM object $E_{T}$ fragmentation function that was discussed in Section 8.4.4 to determine the $E_{T}$ of the plug object that is used in the invariant mass calculation. The second scenario involves a central electron, a plug EM object and plug jet. Again, the plug fake rate is applied for a jet of that $E_{T}$ and $\eta$, and the jet $E_{T} \rightarrow$ plug EM object $E_{T}$ fragmentation function is applied to the $E_{T}$ of the jet. The final possibility involves a central electron, plug EM object and central jet. Here the central photon fake rate is applied for the $E_{T}$ of the jet, where the expected photon $E_{T}$ is computed from the jet $E_{T} \rightarrow \gamma E_{T}$ fragmentation function.

\subsection{3 $Z(\rightarrow e e)+$ jet Background Prediction}

The integrated background from $Z(\rightarrow e e)+$ jet background is $0.24 \pm 0.03($ stat $)$ events, where $0.016 \pm 0.003($ stat $)$ are central and $0.22 \pm 0.03($ stat $)$ are plug. The integrated number of $e \gamma$ combinations is $0.48 \pm 0.05$ (stat), where $0.032 \pm 0.004$ (stat) are central and $0.44 \pm 0.05$ are plug.

\subsection{4 $Z(\rightarrow e e)+$ jet Background Systematics}

The primary source of systematic error on the $Z(\rightarrow e e)+$ jet background is due to the errors on the photon fake rate (Section 8.3) and plug EM object fake rate (Section 8.4). The maximum number of $Z(\rightarrow e e)+$ jet events is 0.47 and the min- 
imum is 0.18 . This brings the total number of predicted $Z(\rightarrow e e)+$ jet events to $0.24 \pm 0.03(\text { stat })_{-0.06}^{+0.23}($ sys $)$. The maximum number $e \gamma$ entries is 0.94 and the minimum is 0.37 . Thus, the number of $e \gamma$ entries is $0.48 \pm 0.05(\text { stat })_{-0.11}^{+0.46}($ sys $)$.

\section{3 $W(\rightarrow e \nu) Z(\rightarrow e e)$ Background}

$W Z$ background, where the $W$ decays to an electron and neutrino and the $Z$ decays leptonically to two electrons, has minimal contribution to the central region search background prediction. For a $W Z$ event to be mistaken for a dielectron + photon candidate, one of the tracks associated with an electron must be "lost". However, this is unlikely in the central detector because the efficiency of COT tracking is greater than $99 \%$. Conversely, $W Z$ production does affect the plug region search because plug electrons and plug photons have no tracking criteria and are treated as identical objects. Hence, plug electrons from $W Z$ events can be interpreted as either electron or photons, and thus contribute to the plug backgrounds.

We study 50,000 PYTHIA $W(\rightarrow e \nu) Z(\rightarrow e e)$ events for events that pass the selection criteria. As was the case for $Z \gamma$, the background distributions predicted by the Monte Carlo must be normalized to the data luminosity using Equations 9.1 and 9.2. Here, $N_{g e n}$ is 50,000 and $\sigma_{M C}$, the generated LO $W Z$ cross-section from PYTHIA, is $9.19 \mathrm{fb}$. Again, we use 1.33 for the K-factor, the NLO QCD correction factor [18].

We weight objects in the same way as described in Section 9.1. 


\subsection{1 $W(\rightarrow e \nu) Z(\rightarrow e e)$ Background Prediction}

The total number of predicted $W Z$ background events is $0.111 \pm 0.002($ stat $)$; specifically, $0.0020 \pm 0.0003($ stat $)$ in the central region and $0.109 \pm 0.002($ stat $)$ in the plug region. The integrated number of e $\gamma$ entries is $0.257 \pm 0.003$ (stat), where $0.0040 \pm 0.0004($ stat) are central events and $0.253 \pm 0.003$ (stat) are plug events.

\subsection{2 $W(\rightarrow e \nu) Z(\rightarrow e e)$ Background Systematics}

It is a reasonable estimation that the systematic errors on the $W Z$ background prediction is similar to that of the $Z \gamma$. So, we conservatively quote $11 \%$ systematic error on the $W Z$ background. Thus, the integrated number of events is $0.111 \pm$ $0.002($ stat $) \pm 0.012($ sys $)$. The integrated number of e $\gamma$ combinations is $0.257 \pm$ $0.003($ stat $) \pm 0.028($ sys $)$.

\section{4 $Z(\rightarrow e e) Z(\rightarrow e e)$ Background}

$Z Z$ production is a source of background when both $Z$ bosons decay to electrons. Just as for $W Z$ production, $Z Z$ is a background when one of the electrons' tracks is not reconstructed in the central region or when one of them is located in the plug region of the detector where tracking information is not used. Again, the central tracking efficiency is greater than $99 \%$, so this background will come primarily from events in which at least one of the electrons is a plug object.

\subsection{1 $Z(\rightarrow e e) Z(\rightarrow e e)$ Background Prediction}

The total number of predicted $Z Z$ background events is $0.0390 \pm 0.0006$ (stat). Specifically, $0.0011 \pm 0.0001$ (stat) in the central region and $0.0378 \pm 0.0006($ stat $)$ in 
the plug region. The integrated number of e $\gamma$ entries is $0.086 \pm 0.001$ (stat), where $0.0023 \pm 0.0002($ stat $)$ are central events and $0.084 \pm 0.001$ (stat) are plug events.

\subsection{2 $Z(\rightarrow e e) Z(\rightarrow e e)$ Background Systematics}

It is a reasonable estimation that the systematic errors on the $Z Z$ background prediction are similar to that of the $Z \gamma$. Hence, an $11 \%$ systematic error is used for the $Z Z$ background. Thus, the integrated number of events is $0.0390 \pm 0.0006($ stat $) \pm$ $0.0043($ sys). The integrated number of e $\gamma$ combinations is $0.086 \pm 0.001($ stat $) \pm$ $0.010($ sys).

\subsection{Multijet Background}

Pure multijet background occurs when one jet fakes the trigger electron and two additional jets fake the second electron and the photon. Events are selected from the signal (electron-triggered) sample.

\subsubsection{Central Region Measurement}

We first discuss the method for predicting the QCD background in the central region search. Events with one loose central, fiducial EM object $\left(E_{T}>25 \mathrm{GeV}\right.$ and $\left.p_{T}>10 \mathrm{GeV}\right)$, and two additional central jets, $j_{1}$ and $j_{2},\left(E_{T}>25 \mathrm{GeV}\right)$ are selected. The EM object must pass the same cuts that were applied to EM objects in the base sample used in the trigger EM object fake rate measurement. Specifically, it must pass the calorimeter isolation, $\frac{E_{H a d}}{E_{E M}}, L_{s h r}$, and $\Delta Z$ cuts.

For the ee $\gamma$ invariant mass plot, each event is weighted by the product of fake 
rates:

$$
w=r_{t}\left[r_{e}\left(j_{1}\right) \cdot r_{\gamma}\left(j_{2}\right)+r_{e}\left(j_{2}\right) \cdot r_{\gamma}\left(j_{1}\right)\right]
$$

where $r_{t}$ is the trigger EM object fake rate evaluated at the $E_{T}$ of the loose EM object, and $r_{e}\left(j_{1}\right)$ and $r_{\gamma}\left(j_{2}\right)$ are the unbiased central jet $\rightarrow$ electron and unbiased central jet $\rightarrow$ photon fake rates, respectively, evaluated at the corresponding jets' $E_{T}$. The two permutations account for both possibilities of faking the second electron and the photon. Additionally, the invariant masses are calculated using the transverse energies specified by the jet $E_{T} \rightarrow$ electron (or photon) $E_{T}$ fragmentation functions.

For the $e \gamma$ invariant mass distribution we consider all possible $e \gamma$ combinations, and thus make three entries per event. Each entry in the $e \gamma$ invariant mass histogram is weighted by:

- $M_{e+j_{1}}$ with weight $w=r_{t} \cdot r_{\gamma}\left(j_{1}\right) \cdot r_{e}\left(j_{2}\right)$

- $M_{e+j_{2}}$ with weight $w=r_{t} \cdot r_{\gamma}\left(j_{2}\right) \cdot r_{e}\left(j_{1}\right)$

- $M_{j_{1}+j_{2}}$ with weight $w=r_{t}\left[r_{e}\left(j_{1}\right) \cdot r_{\gamma}\left(j_{2}\right)+r_{e}\left(j_{2}\right) \cdot r_{\gamma}\left(j_{1}\right)\right]$

\subsubsection{Plug Region Measurement}

The ee $\gamma$ invariant mass distribution entries have weight according to the type of event. If $j_{1}$ is central and $j_{2}$ is plug, $w=r_{t} \cdot\left[r_{e}\left(j_{1}\right) \cdot r_{p}\left(j_{2}\right)+r_{\gamma}\left(j_{1}\right) \cdot r_{p}\left(j_{2}\right)\right]$. If $j_{2}$ is central and $j_{1}$ is plug, $w=r_{t} \cdot\left[r_{e}\left(j_{2}\right) \cdot r_{p}\left(j_{1}\right)+r_{\gamma}\left(j_{2}\right) \cdot r_{p}\left(j_{1}\right)\right]$. If both $j_{1}$ and $j_{2}$ are plug jets, $w=r_{t} \cdot r_{p}\left(j_{1}\right) \cdot r_{p}\left(j_{2}\right)$. The e $\gamma$ invariant mass distribution entries have weights as follows:

- if $j_{1}$ is central and $j_{2}$ is plug 
- $M_{e_{1}+j_{1}}$ with weight $w=r_{t} \cdot r_{\gamma}\left(j_{1}\right) \cdot r_{p}\left(j_{2}\right)$

- $M_{e_{1}+j_{2}}$ with weight $w=r_{t} \cdot r_{e}\left(j_{1}\right) \cdot r_{p}\left(j_{2}\right)$

- $M_{j_{1}+j_{2}}$ with weight $w=r_{t} \cdot\left[r_{e}\left(j_{1}\right) \cdot r_{p}\left(j_{2}\right)+r_{\gamma}\left(j_{1}\right) \cdot r_{p}\left(j_{2}\right)\right]$

- if $j_{2}$ is central and $j_{1}$ is plug

- $M_{e_{1}+j_{1}}$ with weight $w=r_{t} \cdot\left[r_{\gamma}\left(j_{2}\right) \cdot r_{p}\left(j_{1}\right)\right]$

- $M_{e_{1}+j_{2}}$ with weight $w=r_{t} \cdot\left[r_{e}\left(j_{2}\right) \cdot r_{p}\left(j_{1}\right)\right]$

- $M_{j_{1}+j_{2}}$ with weight $w=r_{t} \cdot\left[r_{e}\left(j_{2}\right) \cdot r_{p}\left(j_{1}\right)+r_{\gamma}\left(j_{2}\right) \cdot r_{p}\left(j_{1}\right)\right]$

- if both $j_{1}$ and $j_{2}$ are plug

- $M_{e_{1}+j_{1}}$ with weight $w=r_{t} \cdot r_{p}\left(j_{1}\right) \cdot r_{p}\left(j_{2}\right)$

- $M_{e_{1}+j_{2}}$ with weight $w=r_{t} \cdot r_{p}\left(j_{1}\right) \cdot r_{p}\left(j_{2}\right)$

- $M_{j_{1}+j_{2}}$ with weight $w=r_{t} \cdot r_{p}\left(j_{1}\right) \cdot r_{p}\left(j_{2}\right)$

Again, the corresponding energy fragmentation functions are applied to the jet $E_{T}$ for the invariant mass calculations.

\subsubsection{Multijet Background Prediction}

The integrated number of multijet events predicted is $0.0271 \pm 0.0006$ (stat), where $0.00085 \pm 0.00001($ stat $)$ are central and $0.0262 \pm 0.0006($ stat $)$ are plug. The integrated number of e $\gamma$ entries is $0.070 \pm 0.001$ (stat), where $0.00170 \pm 0.00002$ (stat) are central and $0.068 \pm 0.001$ (stat) are plug. 


\subsubsection{Multijet Background Prediction Systematics}

The largest source of uncertainty in the multijet background prediction is due to the error on the fake rates. The maximum number of multijet events which fake the signal is 0.0604 and the minimum is 0.0174 events. The integrated number of events from multijet background is therefore $0.0271 \pm 0.0006(\text { stat })_{-0.0097}^{+0.0333}($ sys $)$. The maximum integrated number of e $\gamma$ entries is 0.162 and the minimum is 0.044 . Thus, the total number of e $\gamma$ entries from multijet background is $0.070 \pm 0.001(\text { stat })_{-0.026}^{+0.092}($ sys $)$.

\section{$9.6 t \bar{t}$ Background}

Top quarks decay almost exclusively to a $W$ boson and a $b$ quark [2]. Thus, $t \bar{t}$ events can be a source of background when one of the $b$ quarks radiates a hard photon and both $W$ bosons decay in the electron channel as in:

$$
q+\bar{q} \rightarrow t\left(\rightarrow W^{+} b \rightarrow e^{+} \nu_{e} b\right)+\bar{t}\left(\rightarrow W^{-} b \rightarrow e^{-} \bar{\nu}_{e} b\right)
$$

The $t \bar{t}$ background was estimated using the "ttopei" sample which is an inclusive PYTHIA $t \bar{t}$ Monte Carlo sample of 683801 events with final state QED photon radiation on. The generated NLO cross-section is $6.7 \mathrm{pb}$. Hence, the Monte Carlo luminosity is $102 \mathrm{nb}$.

\subsection{1 $t \bar{t}$ Background Prediction}

The total number of predicted $t \bar{t}$ background events is $0.015 \pm 0.005$ (stat), $0.002 \pm$ 0.002 (stat) in the central region and $0.013 \pm 0.005$ (stat) in the plug region. The integrated number of e $\gamma$ entries is $0.030 \pm 0.007$ (stat), where $0.004 \pm 0.003$ (stat) are 
central events and $0.026 \pm 0.007$ (stat) are plug events.

\subsection{2 $t \bar{t}$ Background Systematics}

It is a reasonable estimation that the systematic errors on the $t \bar{t}$ background prediction is similar to that of the $Z \gamma$. So, an $11 \%$ systematic error is applied to the $t \bar{t}$ background. Thus, the integrated number of events is $0.015 \pm 0.005$ (stat) \pm 0.002 (sys). The integrated number of e $\gamma$ combinations is $0.030 \pm 0.007$ (stat) \pm $0.003($ sys $)$.

\subsection{Diphoton + jet Background}

Diphoton + jet production is another source of background which may mimic the search signal when the photon converts and is identified as an electron. Then the second photon is detected as such and the jet is mis-identified as an electron.

This background is measured using a sample of 472965 PYTHIA diphoton events. We rely on PYTHIA's initial state radiation to produce jets as they are in data. The generated LO diphoton cross-section is $92.98 \mathrm{pb}$, and 1.3 is used for the K-factor. Equations 9.1 and 9.2 are used to normalize the Monte Carlo luminosity to the data.

\subsubsection{Central Region}

For measuring the background contribution in the central region, we look for events with a central electron, central photon, and central jet. For such events, the entries in the invariant mass distributions are weighted by the unbiased central jet $\rightarrow$ electron fake rate. The energy resolution smearing corrections are applied to the 
electron and photon energies. The jet energy is scaled by the central jet $E_{T} \rightarrow$ electron $E_{T}$ fragmentation function.

\subsubsection{Plug Region}

The measurement of diphoton + jet production in the plug detector region is done by requiring one of three possibilities:

- A central electron, central photon, and a plug jet. If this is the case, we smear the electron and photon energies as we do for all simulated central objects. The event is weighted by the plug fake rate at the jet $E_{T}$ and $\eta_{d}$. The invariant masses are calculated using the $E_{T}$ specified by the jet $\rightarrow$ plug EM object fragmentation function.

- A central electron, plug EM object and central jet. In this case, we smear the electron and plug EM object energies as usual. Since this is diphoton + jet production, we assume the plug EM object is a photon and so we weight the central jet by the jet $\rightarrow$ electron fake rate. The jet $E_{T}$ is scaled according to the central jet $\rightarrow$ electron $E_{T}$ fragmentation function.

- A central electron, plug EM object and plug jet. For this case, we again smear the electron and plug EM object energies. We weight the event by the jet $\rightarrow$ plug EM object fake rate. Additionally, the plug jet $E_{T} \rightarrow$ plug EM object $E_{T}$ fragmentation function is applied.

\subsubsection{Diphoton + jet Background Prediction}

The integrated number of events that diphoton + jet background can mimic is $0.008 \pm 0.001$ (stat). $0.00013 \pm 0.00005$ (stat) events are predicted in the central 
region; the remaining, and majority, have at least one plug EM object.

The integrated number of entries in the $M_{e \gamma}$ distribution is $0.020 \pm 0.002$ (stat), with $0.00025 \pm 0.00007$ being central events and the remainder being plug events.

\subsubsection{Diphoton + jet Background Systematics}

The primary source of systematic error on diphoton + jet production is due to the uncertainty in the fake rate measurements. We find the systematic error on the total number of predicted events to be ${ }_{-0.003}^{+0.004}$, so the diphoton + jet contribution is $0.008 \pm$ $0.001(\text { stat })_{-0.003}^{+0.004}($ sys $)$. The systematic error on the integrated $M_{e \gamma}$ distribution is ${ }_{-0.004}^{+0.022}$, so the integrated number of entries in the $M_{e \gamma}$ distribution is $0.020 \pm$ $0.002(\text { stat })_{-0.004}^{+0.022}$.

\section{8 $W(\rightarrow e \nu)+$ jets Background}

Background due to $W(\rightarrow e \nu)+$ jets occurs when one jet fakes an electron and another fakes a photon. This source of background is measured from the signal (electrontriggered) dataset by applying the appropriate fake rates to jets in events with one central electron and two additional jets $\left(j_{1}\right.$ and $\left.j_{2}\right)$ with $E_{T}>25 \mathrm{GeV}$ and event $\not \mathbb{H}_{T}>25 \mathrm{GeV}$.

\subsubsection{Central Region Measurement}

Each $W+$ jets candidate is entered into the ee $\gamma$ distribution by weighting events with the product of fake rates: $w=r_{e}\left(j_{1}\right) \cdot r_{\gamma}\left(j_{2}\right)+r_{e}\left(j_{2}\right) \cdot r_{\gamma}\left(j_{1}\right)$. For the e $\gamma$ invariant mass distribution, each event has entries with the following weights:

- $M_{e+j_{1}}$ with weight $w=r_{\gamma}\left(j_{1}\right) \cdot r_{e}\left(j_{2}\right)$ 
- $M_{e+j_{2}}$ with weight $w=r_{\gamma}\left(j_{2}\right) \cdot r_{e}\left(j_{1}\right)$

- $M_{j_{1}+j_{2}}$ with weight $w=r_{e}\left(j_{1}\right) \cdot r_{\gamma}\left(j_{2}\right)+r_{e}\left(j_{2}\right) \cdot r_{\gamma}\left(j_{1}\right)$

As usual, the appropriate energy fragmentation functions are applied to the jet $E_{T}$ for the invariant mass calculations.

\subsubsection{Plug Region Measurement}

The ee $\gamma$ invariant mass distribution entries have weight according to the type of event. If $j_{1}$ is central and $j_{2}$ is plug, $w=r_{e}\left(j_{1}\right) \cdot r_{p}\left(j_{2}\right)+r_{\gamma}\left(j_{1}\right) \cdot r_{p}\left(j_{2}\right)$. If $j_{2}$ is central and $j_{1}$ is plug, $w=r_{e}\left(j_{2}\right) \cdot r_{p}\left(j_{1}\right)+r_{\gamma}\left(j_{2}\right) \cdot r_{p}\left(j_{1}\right)$. If both $j_{1}$ and $j_{2}$ are plug jets, $w=r_{p}\left(j_{1}\right) \cdot r_{p}\left(j_{2}\right)$. The e $\gamma$ invariant mass distribution entries have weights as follows:

- if $j_{1}$ is central and $j_{2}$ is plug

- $M_{e_{1}+j_{1}}$ with weight $w=r_{\gamma}\left(j_{1}\right) \cdot r_{p}\left(j_{2}\right)$

- $M_{e_{1}+j_{2}}$ with weight $w=r_{e}\left(j_{1}\right) \cdot r_{p}\left(j_{2}\right)$

- $M_{j_{1}+j_{2}}$ with weight $w=r_{e}\left(j_{1}\right) \cdot r_{p}\left(j_{2}\right)+r_{\gamma}\left(j_{1}\right) \cdot r_{p}\left(j_{2}\right)$

- if $j_{2}$ is central and $j_{1}$ is plug

- $M_{e_{1}+j_{1}}$ with weight $w=r_{\gamma}\left(j_{2}\right) \cdot r_{p}\left(j_{1}\right)$

- $M_{e_{1}+j_{2}}$ with weight $w=r_{e}\left(j_{2}\right) \cdot r_{p}\left(j_{1}\right)$

- $M_{j_{1}+j_{2}}$ with weight $w=r_{e}\left(j_{2}\right) \cdot r_{p}\left(j_{1}\right)+r_{\gamma}\left(j_{2}\right) \cdot r_{p}\left(j_{1}\right)$

- if both $j_{1}$ and $j_{2}$ are plug

- $M_{e_{1}+j_{1}}$ with weight $w=r_{p}\left(j_{1}\right) \cdot r_{p}\left(j_{2}\right)$ 
- $M_{e_{1}+j_{2}}$ with weight $w=r_{p}\left(j_{1}\right) \cdot r_{p}\left(j_{2}\right)$

- $M_{j_{1}+j_{2}}$ with weight $w=r_{p}\left(j_{1}\right) \cdot r_{p}\left(j_{2}\right)$

Again, the corresponding energy fragmentation functions are applied to the jet $E_{T}$ for the invariant mass calculations.

\subsection{3 $W(\rightarrow e \nu)+$ Jets Background Prediction}

The predicted integrated $W(\rightarrow e \nu)+$ jets background is $0.0041 \pm 0.0002($ stat $)$ events, where $0.00029 \pm 0.00001$ (stat) are central and $0.0038 \pm 0.0002$ (stat) are plug. The integrated number of $M_{e \gamma}$ combinations is $0.0098 \pm 0.0004$ (stat), where $0.00057 \pm$ 0.00001 (stat) are central and $0.0092 \pm 0.0004$ (stat) are plug.

\subsection{4 $W(\rightarrow e \nu)+$ Jets Background Systematics}

The primary source of systematic error is due to the fake rate errors discussed in Chapter 8. The maximum integrated number of $W(\rightarrow e \nu)+$ jets events is 0.0096 and the minimum is 0.0024 , bringing the predicted number of events to $0.0041 \pm$

$0.0002(\text { stat })_{-0.0017}^{+0.0055}($ sys $)$. The maximum number of predicted e $\gamma$ entries is 0.025 and the minimum is 0.0056 . The integrated number of e $\gamma$ combinations from $W(\rightarrow$ $e \nu)+$ jets events is $0.0098 \pm 0.0004(s t a t)_{-0.0042}^{+0.015}(s y s)$.

\subsection{Background Prediction from All Sources}

Table 9.2 shows a summary of the integrated number of background events predicted, along with the statistical and systematic errors. Similarly, Table 9.2 shows a summary of the integrated number of e $\gamma$ entries predicted and the associated statistical and systematic errors. 
The total statistical and systematic errors are obtained in the following manner. To get the total statistical error, the statistical errors from each source are added in quadrature. The $Z \gamma, W Z, Z Z$, and $t \bar{t}$ systematic errors are added linearly since they are correlated errors associated with the simulation. The systematic errors on the backgrounds associated with fake rates $(Z+$ jet, multijet, diphoton+jet, and $W+$ jet production) are added linearly because the errors are all due to the uncertainties in the fake rate measurements. To get the total systematic error, the combined systematic error from $Z \gamma, W Z, Z Z$, and $t \bar{t}$ is added in quadrature with the systematic error from the fake backgrounds.

\begin{tabular}{|l|c|c|c|}
\hline $\begin{array}{l}\text { Background } \\
\text { Source }\end{array}$ & $\begin{array}{c}\text { Integrated } \\
\text { Number } \\
\text { of Events }\end{array}$ & $\begin{array}{c}\text { Statistical } \\
\text { Error }\end{array}$ & $\begin{array}{c}\text { Systematic } \\
\text { Error }\end{array}$ \\
\hline \hline$Z \gamma$ & 2.584 & 0.076 & 0.276 \\
\hline$Z(\rightarrow e e)+$ jet & 0.238 & 0.032 & ${ }_{-0.056}^{+0.232}$ \\
\hline$W(\rightarrow e \nu) Z(\rightarrow e e)$ & 0.111 & 0.002 & 0.012 \\
\hline$Z(\rightarrow e e) Z(\rightarrow e e)$ & 0.0390 & 0.0006 & 0.0043 \\
\hline Multijet & 0.0271 & 0.0006 & ${ }_{-0.0097}^{+0.0332}$ \\
\hline$t \bar{t}$ & 0.015 & 0.005 & 0.002 \\
\hline$\gamma \gamma+$ jet & 0.008 & 0.001 & ${ }_{-0.003}^{+0.004}$ \\
\hline$W(\rightarrow e \nu)+$ jets & 0.0041 & 0.0002 & ${ }_{-0.0017}^{+0.0055}$ \\
\hline \hline Total & 3.03 & 0.08 & ${ }_{-0.30}^{+0.40}$ \\
\hline
\end{tabular}

Table 9.2: Summary of the background prediction for the integrated number of events and associated errors.

Figure 9.1(a) shows the individual background contributions due to each source as a function of $M_{e \gamma}$, and Figure $9.1(\mathrm{~b})$ shows the summed ${ }^{1}$ backgrounds in the $M_{e \gamma}$ distribution. Figure 9.2(a) shows the individual background contributions in the $M_{e e \gamma}$ distribution, and Figure $9.2(\mathrm{~b})$ shows the stacked backgrounds in the $M_{e e \gamma}$

\footnotetext{
1 "Summed" or "stacked" backgrounds means that each background has been added to the one below it. The backgrounds are stacked in order according to the size of the background contribution, starting with the smallest.
} 


\begin{tabular}{|l|c|c|c|}
\hline $\begin{array}{l}\text { Background } \\
\text { Source }\end{array}$ & $\begin{array}{c}\text { Integrated } \\
\text { Number } \\
\text { of } e \gamma \text { Entries }\end{array}$ & $\begin{array}{c}\text { Statistical } \\
\text { Error }\end{array}$ & $\begin{array}{c}\text { Systematic } \\
\text { Error }\end{array}$ \\
\hline \hline$Z \gamma$ & 5.560 & 0.111 & 0.592 \\
\hline$Z(\rightarrow e e)+$ jet & 0.475 & 0.046 & ${ }_{-0.112}^{+0.463}$ \\
\hline$W(\rightarrow e \nu) Z(\rightarrow e e)$ & 0.257 & 0.003 & 0.028 \\
\hline$Z(\rightarrow e e) Z(\rightarrow e e)$ & 0.086 & 0.001 & 0.010 \\
\hline Multijet & 0.070 & 0.001 & ${ }_{-0.026}^{+0.092}$ \\
\hline$t \bar{t}$ & 0.030 & 0.007 & 0.003 \\
\hline$\gamma \gamma+$ jet & 0.020 & 0.002 & ${ }_{-0.004}^{+0.022}$ \\
\hline$W(\rightarrow e \nu)+$ jets & 0.0098 & 0.0004 & ${ }_{-0.0042}^{+0.0146}$ \\
\hline \hline Total & 6.51 & 0.12 & ${ }_{-0.65}^{+0.87}$ \\
\hline
\end{tabular}

Table 9.3: Summary of integrated number of $e \gamma$ entries background prediction and associated errors.

distribution. Figure 9.3 has the total background contribution as a function of $M_{e *}$. The total background has been fitted for masses greater than $100 \mathrm{GeV}$ and the associated systematic error as a function of $e^{*}$ mass is shown.

In Section 10.6, the integrated background distributions from high mass to low mass are discussed and compared to the observed data.

\subsection{Effect of Dielectron Invariant Mass Cut}

To reduce the background due to initial state radiation $Z(\rightarrow e e)+\gamma$ production and $Z(\rightarrow e e)+$ jet production, this analysis requires the dielectron invariant mass of each event to be less than $81 \mathrm{GeV}$ or greater than $101 \mathrm{GeV}$. If the event contains two plug EM objects (i.e. we can not discern which is an electron and which is a photon), we require that both possible $M_{e e}$ combinations pass this cut.

Figure 9.4 shows the invariant mass distributions of the electron pairs for the $Z+\gamma$ and $Z+$ jet backgrounds. The arrows show the location of the $M_{e e}$ cut. We 


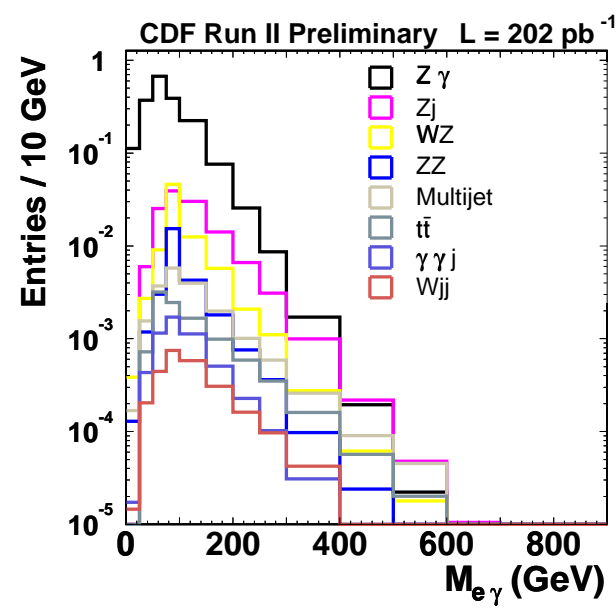

(a)

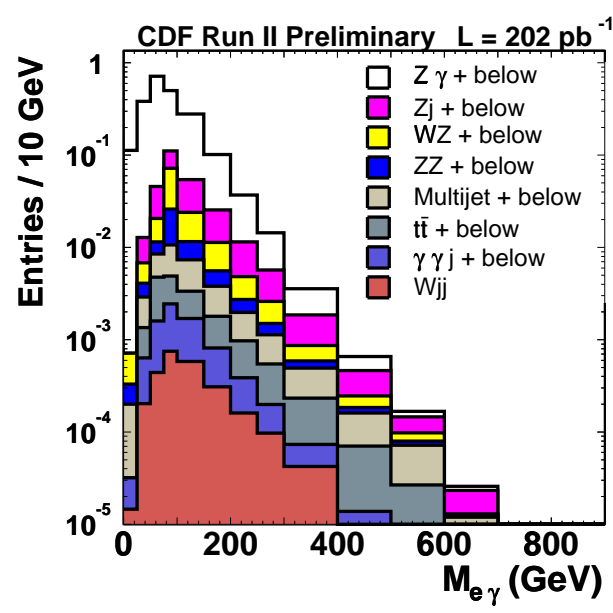

(b)

Figure 9.1: Figure (a) shows the individual background contributions from each source for the $M_{e \gamma}$ distribution. Figure (b) shows the summed background contributions for the $M_{e \gamma}$ distribution.

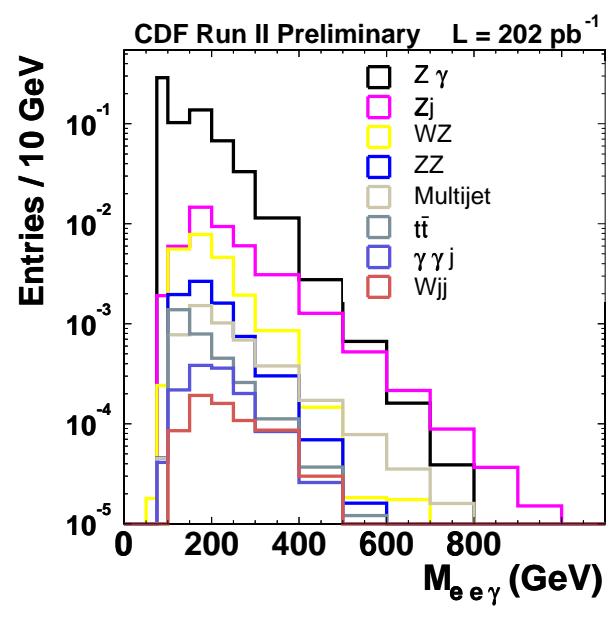

(a)

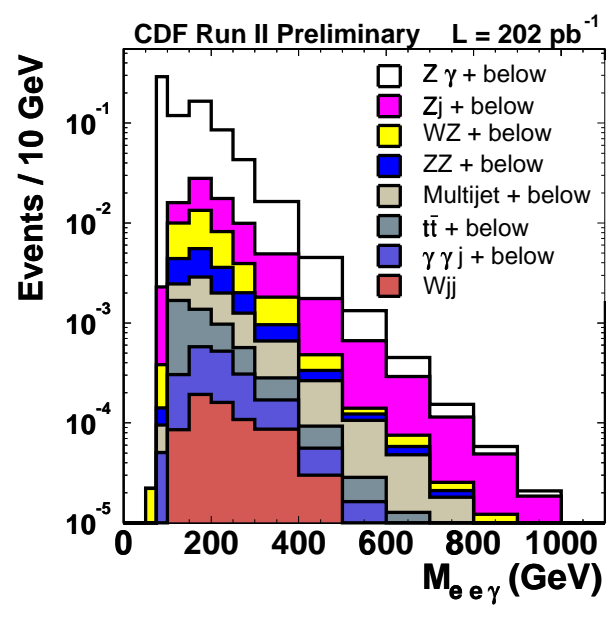

(b)

Figure 9.2: Figure (a) shows the individual contribution from each source of background for the $M_{e e \gamma}$ distribution. Figure (b) shows the summed background contributions for the $M_{e e \gamma}$ distribution. 


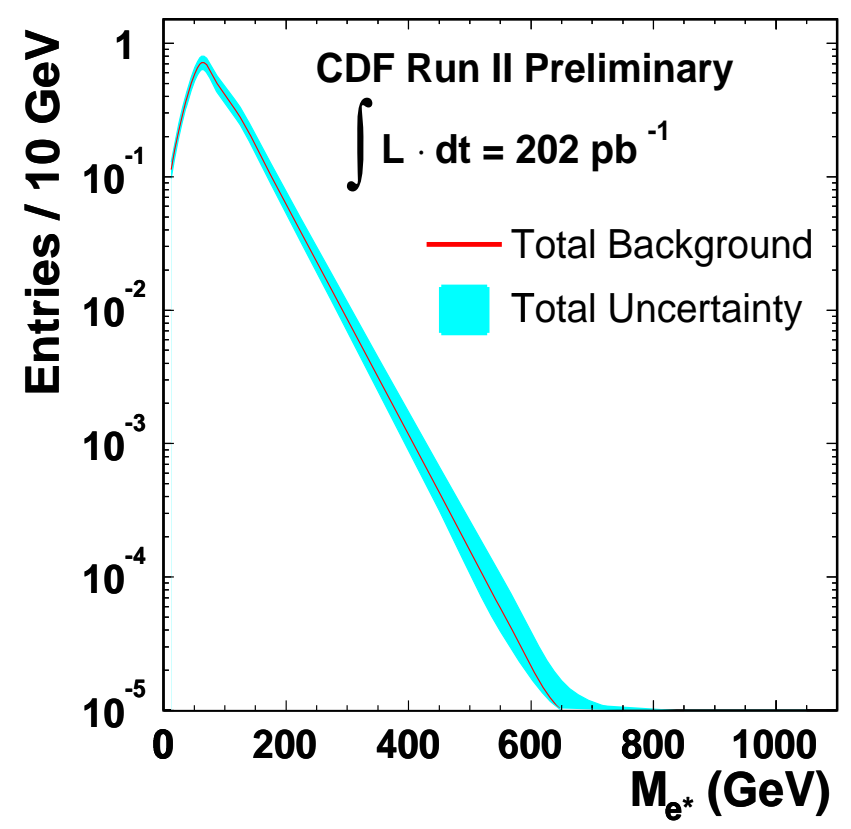

Figure 9.3: Total background contribution from all sources. The red curve is the central value and the blue band is the error band.

expect $\sim 2.8$ events outside the mass window and $\sim 6$ inside the mass window from $Z+\gamma$ and $Z+$ jet events. Hence, the cut significantly reduced the backgrounds with the two leading backgrounds being reduced by $\sim 72 \%$. 


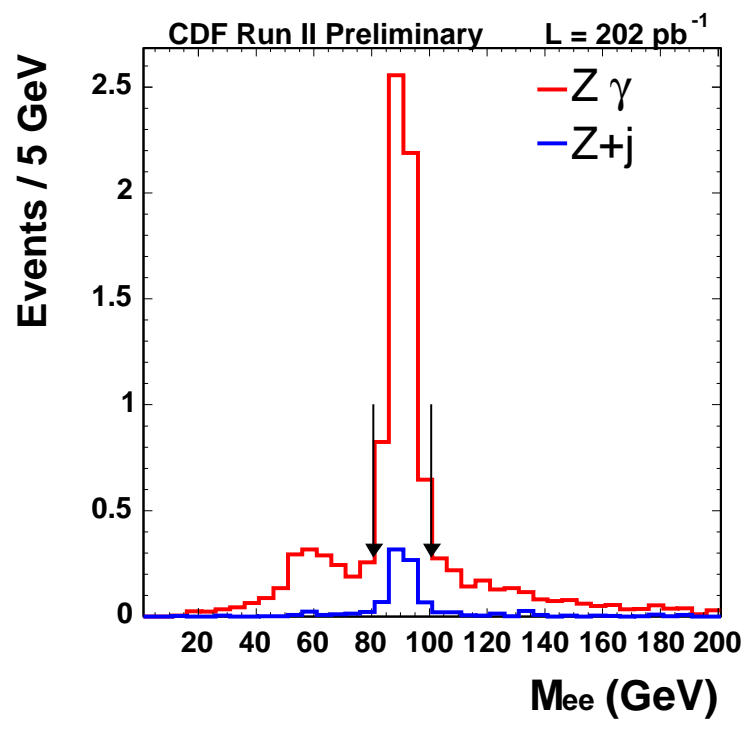

Figure 9.4: Dielectron invariant mass spectra for the two leading backgrounds, $Z+\gamma$ and $Z+$ jet. Events with $81<M_{e e}<101 \mathrm{GeV}$ are rejected. 


\section{Chapter 10}

\section{Discussion of Candidate Events}

\subsection{Introduction}

Having understood the total signal acceptance of the excited electron models and predicted the total background as a function of $e^{*}$ mass, approximately $202 p b^{-1}$ of data are searched for events which pass the selection requirements. Three candidate events are found. Run 144674, event 4143240 is a central-plug-plug candidate. Run 147806, event 1167222 has two central electrons and one plug EM object. Run 167866, event 443088 has two central electrons and one central photon.

\subsection{Phoenix Tracking}

The events with plug EM objects do not have tracking requirements for identification. However, the particle may have traversed a portion of the detector with silicon tracking coverage. To better understand these events, we study the plug EM objects using a tracking method called Phoenix Tracking [31], [32].

Phoenix Tracking uses the location of electromagnetic clusters in the showermax detector, the event position of the primary vertex, and the transverse energy of the EM object to create the helical properties of two possible seed tracks: one 
for a negatively charged electron and one for a positron. These two seed tracks are compared and matched to hits in the silicon detector to determine if a charged particle traveled through the region. If both positive and negative seed tracks are matched to silicon hits, the best $\chi^{2} /$ d.o.f. fit is used to determine the "correct" track and, from that, the charge. A more detailed explanation is documented in [31].

\subsection{Run 144674, Event 4143240}

Run 144674, event 4143240 contains one central electron and two plug EM objects. Details for this event are found in Table 10.1 and Figures 10.1(a) and 10.1(b). The $\mathscr{E}_{T}$, calculated from the beam constrained track vertex of the electron, is $21 \mathrm{GeV}$.

The display in Figure 10.1(a) shows two well-defined tracks in the event. The track reconstructed in the central region is positively charged. The Phoenix Tracking module confirms the observation of one plug track (in this case, $p_{2}$ ). Figure 10.2(a) shows both possible positive and negative helical trajectories for $p_{2}$. It also shows the silicon hits and reconstructed track that best matches the negatively charged seed track. However, for $p_{1}$, no track is reconstructed by the phoenix module. The reason for this is shown in Figure 10.2(b) where the location of the event vertex, $\sim 40 \mathrm{~cm}$, and the trajectory of $p_{1}$ put its location outside the region of good silicon coverage.

Thus, this event contains one central positron (e), one plug electron $\left(p_{2}\right)$ and one plug EM object $\left(p_{1}\right)$ with unknown charge properties. Assuming $p_{1}$ is a photon, we consider the possibility that this event is a $Z+\gamma$ event, which is the largest predicted background. The $61 \mathrm{GeV} e+p_{2}$ invariant mass indicate a possible final state radiation $Z+\gamma$ event. However, the high $318 \mathrm{GeV}$ three body mass $\left(M_{e p_{1} p_{2}}\right)$ make this highly unlikely. Given Figures 9.1 and 9.2 , this is most likely a $Z+\gamma$ 
event with an initial state radiation photon.

\begin{tabular}{|l|c|c|c|}
\hline & e & $p_{1}$ & $p_{2}$ \\
\hline$E_{T}(\mathrm{GeV})$ & 37 & 71 & 48 \\
\hline$\eta_{d}$ & -0.87 & 1.48 & -1.47 \\
\hline$\eta$ & -1.01 & 1.27 & -1.65 \\
\hline$\phi$ & 0.62 & 4.05 & 2.02 \\
\hline \hline$M_{e p_{1}}$ & $M_{e p_{2}}$ & $M_{p_{1} p_{2}}$ & $M_{e p_{1} p_{2}}$ \\
\hline $176 \mathrm{GeV}$ & $61 \mathrm{GeV}$ & $257 \mathrm{GeV}$ & $318 \mathrm{GeV}$ \\
\hline
\end{tabular}

Table 10.1: Detailed information for run 144674, event 4143240. 


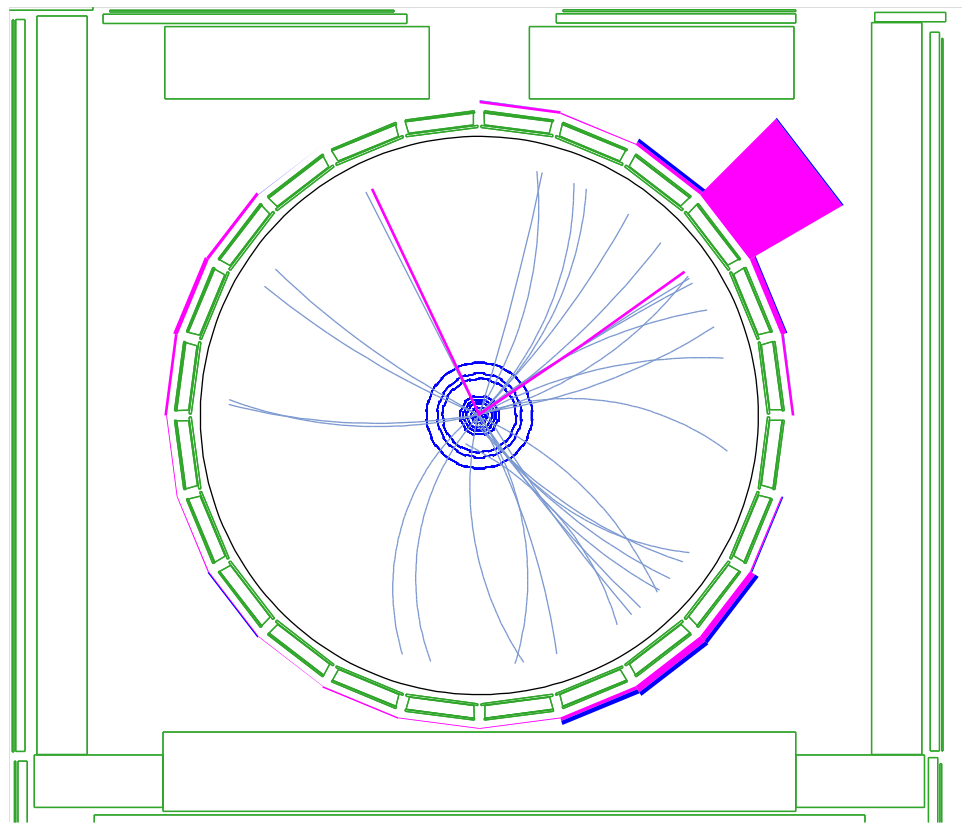

(a)

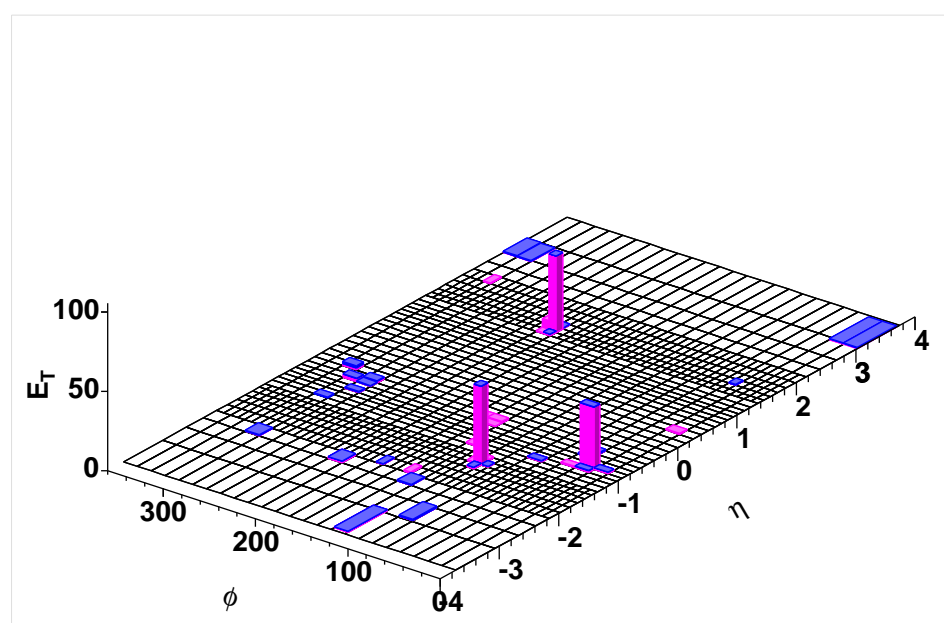

(b)

Figure 10.1: Event displays for Run $=144674$, Event $=4143240$. Figure (a) shows the COT event display with track $p_{T}>0.5 \mathrm{GeV}$. Figure (b) shows the calorimeter physics towers event display with tower $E_{T}>0.5 \mathrm{GeV}$. 


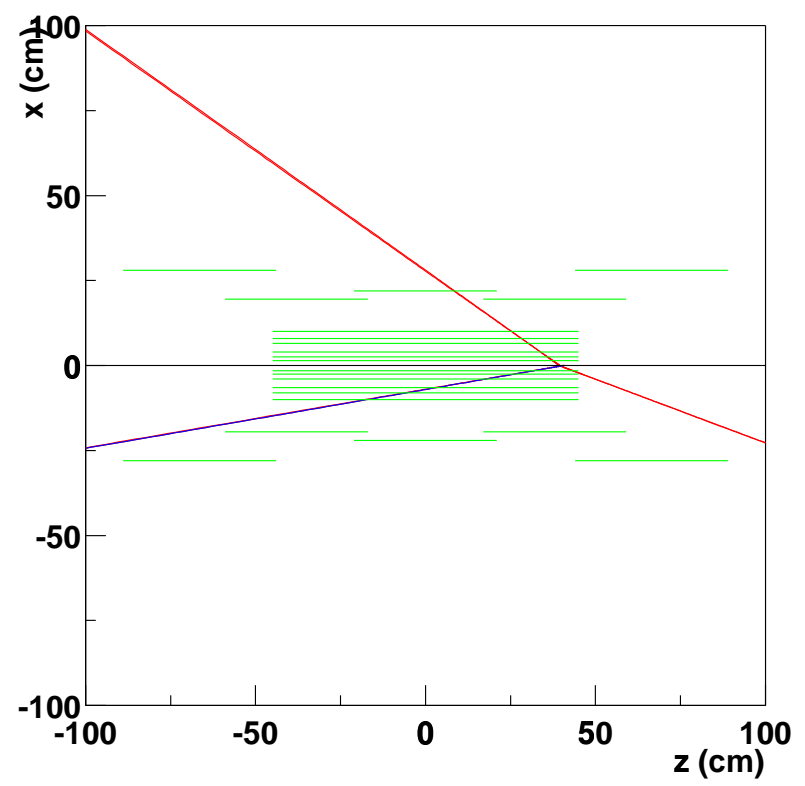

(a)

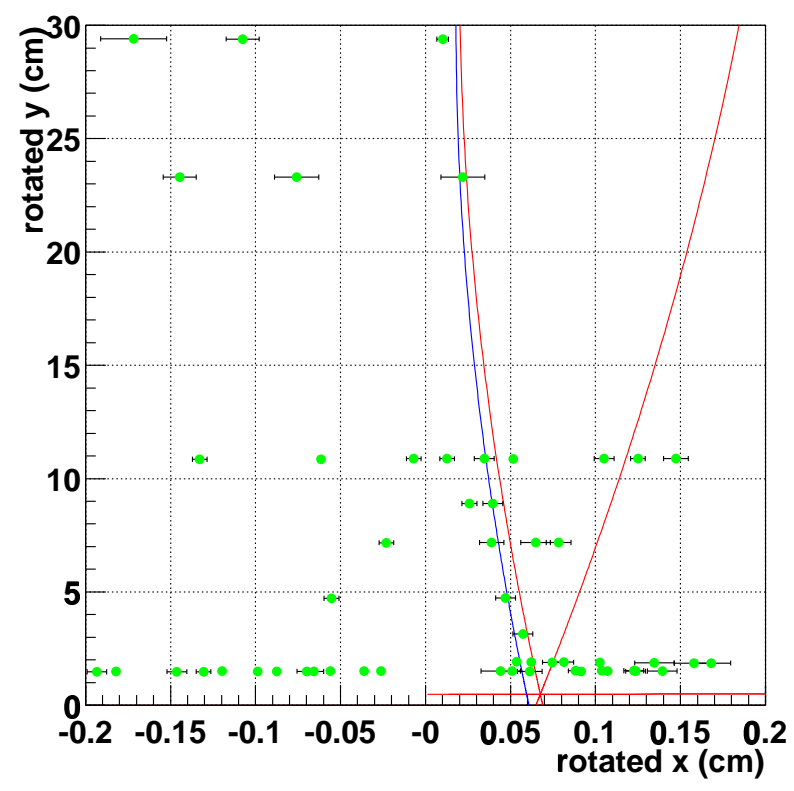

(b)

Figure 10.2: Phoenix track displays for $r u n=144674$, event $=4143240$. Figure (a) shows both $X$ vs $Z$ hypotheses (the red curves) and the best fit (negative charge, blue curve) for $p_{2}$. Figure (b) $x-z$ display shows the projected trajectories of the objects. 


\subsection{Run 147806, Event 1167222}

Run 147806, event 1167222 has two central electrons $\left(e_{1}\right.$ and $\left.e_{2}\right)$ and one plug EM object $\left(p_{1}\right)$ which pass the event requirements. This event also has a fourth EM object which is located in the plug calorimeter with $E_{T}=26 \mathrm{GeV}$. This object almost passes the plug EM object selection criteria with associated identification variables calorimeter isolation ratio $=0.107, \operatorname{PEM} \chi^{2}=9.1$, and $\frac{E_{H a d}}{E_{E M}}=0.02$.

Details for this event are found in Table 10.2 and Figures 10.3 (a) and 10.3 (b). The event $\mathscr{H}_{T}$ is $13 \mathrm{GeV}$, as calculated from the beam constrained vertex of electron $e_{1}$.

\begin{tabular}{|l|c|c|c|c|}
\hline & $e_{1}$ & $e_{2}$ & $p_{1}$ & $p_{2}$ \\
\hline$E_{T}(\mathrm{GeV})$ & 44 & 42 & 46 & 26 \\
\hline$\eta_{d}$ & 0.81 & -0.19 & 1.46 & 1.51 \\
\hline$\eta$ & 0.83 & -0.17 & 1.46 & 1.53 \\
\hline$\phi$ & 3.64 & 1.96 & 0.92 & 5.08 \\
\hline
\end{tabular}

\begin{tabular}{|l|c|c|c|}
\hline$M_{e_{1} e_{2}}$ & $M_{e_{1} p_{1}}$ & $M_{e_{2} p_{1}}$ & $M_{e_{1} e_{2} p_{1}}$ \\
\hline $78 \mathrm{GeV}$ & $92 \mathrm{GeV}$ & $92 \mathrm{GeV}$ & $152 \mathrm{GeV}$ \\
\hline
\end{tabular}

\begin{tabular}{|l|c|c|c|c|c|c|}
\hline$M_{e_{1} p_{2}}$ & $M_{e_{2} p_{2}}$ & $M_{p_{1} p_{2}}$ & $M_{e_{1} e_{2} p_{2}}$ & $M_{e_{1} p_{1} p_{2}}$ & $M_{e_{2} p_{1} p_{2}}$ & $M_{e_{1} e_{2} p_{1} p_{2}}$ \\
\hline $51 \mathrm{GeV}$ & $91 \mathrm{GeV}$ & $60 \mathrm{GeV}$ & $131 \mathrm{GeV}$ & $121 \mathrm{GeV}$ & $143 \mathrm{GeV}$ & $194 \mathrm{GeV}$ \\
\hline
\end{tabular}

Table 10.2: Event information for run=147806, event $=1167222 . p_{2}$ is the fourth EM object in the event.

This is a very interesting and clean event in which both $e_{1}$ and $e_{2}$ are positively charged. The plug EM objects are located within the region of the silicon tracking detector and have silicon tracks associated with them, as shown in Figure 10.3(a). The Phoenix Tracking information tells us that both plug EM objects are negatively charged [32]. Although $p_{2}$ has poor isolation, it is very close to passing the selection criteria; thus, this event appears to have 4 electrons, a very rare signature. The 


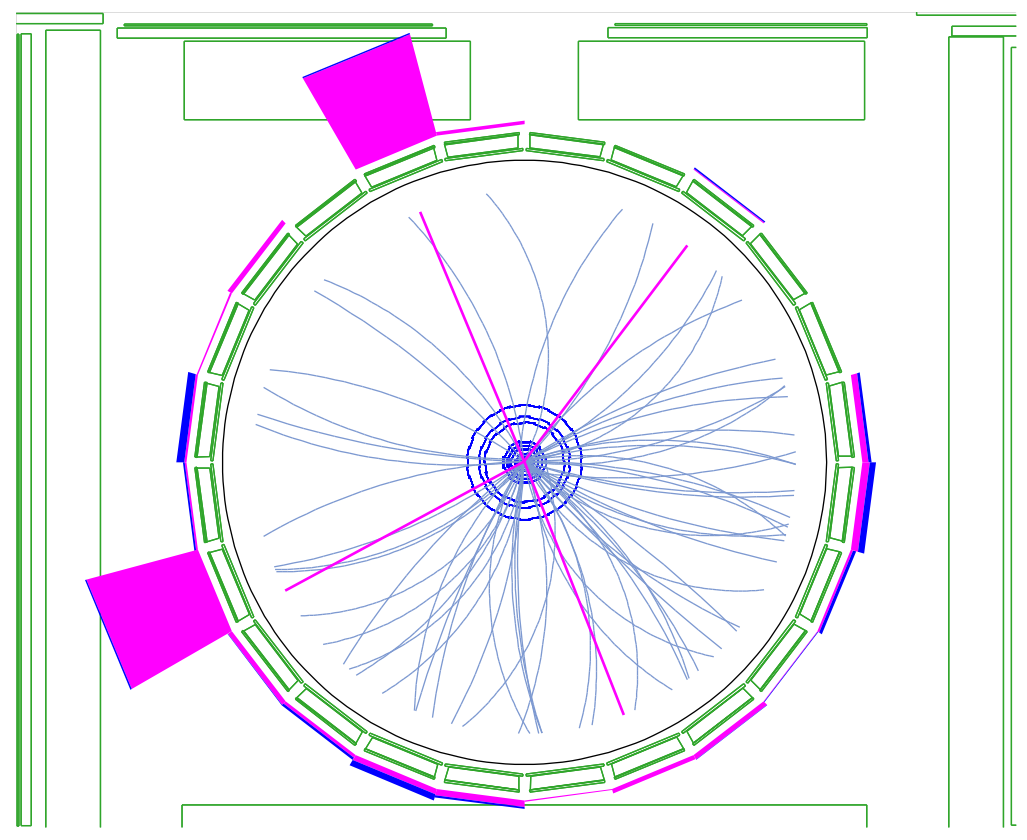

(a)

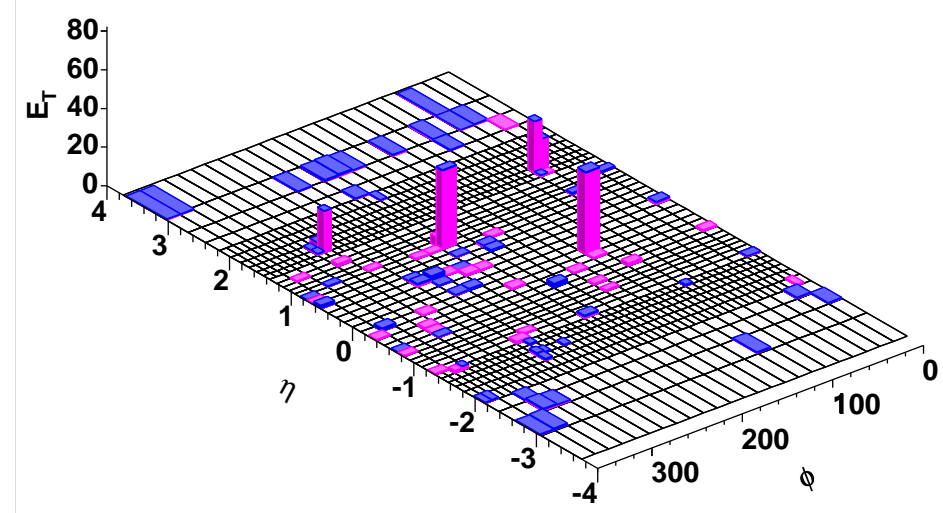

(b)

Figure 10.3: Event displays for $r u n=147806$, event $=1167222$. Figure (a) shows the COT event display with track $p_{T}>0.5 \mathrm{GeV}$. Figure (b) shows the calorimeter physics towers event display with tower $E_{T}>0.5 \mathrm{GeV}$. 
$Z(\rightarrow e e) Z(\rightarrow e e)$ standard model process where both $Z$ bosons decay in the electron channel would produce this signal. The invariant masses of $M_{e_{1} p_{1}}$ and $M_{e_{2} p_{2}}$ are both near $91 \mathrm{GeV}$, supporting the idea that this is a $Z Z$ event.

The selection criteria used in this analysis predicted $0.04 Z(\rightarrow e e) Z(\rightarrow e e)$ events in $202 p b^{-1}$ of data. The likelihood of observing this type of event is $\sim 3.8 \%$.

\subsection{Run 167866, Event 443088}

Run 167866, event 443088 has two oppositely charged central electrons $\left(e_{1}\right.$ and $\left.e_{2}\right)$ and one central photon $(\gamma)$. Details of this event can be found in Table 10.3 and the event displays are Figures 10.4(a) and 10.4(b). The event has $\mathbb{E}_{T}=11 \mathrm{GeV}$, as calculated from the beam constrained track vertex of the maximum $E_{T}$ electron $\left(e_{1}\right)$. The LEGO plot of Figure 10.4 (b) shows a fourth hadronic jet in the event with $E_{T} \sim 25 \mathrm{GeV}, \eta_{d}=-0.42$, and $\phi=3.16$.

This event has exceptionally high dielectron invariant mass of $256 \mathrm{GeV}$, along with unusually high electron and photon energies. Additionally, $M_{e_{1} \gamma}$ and $M_{e_{1} e_{2} \gamma}$ are $220 \mathrm{GeV}$ and $344 \mathrm{GeV}$, respectively. These properties, along with the presence of an additional hadronic jet, make this event difficult to categorize as one of the considered backgrounds. With the information available, it is difficult to make a statement regarding what type of event this is. It could possibly be a $Z+\gamma+$ jet event or a $Z+2$ jet event. 


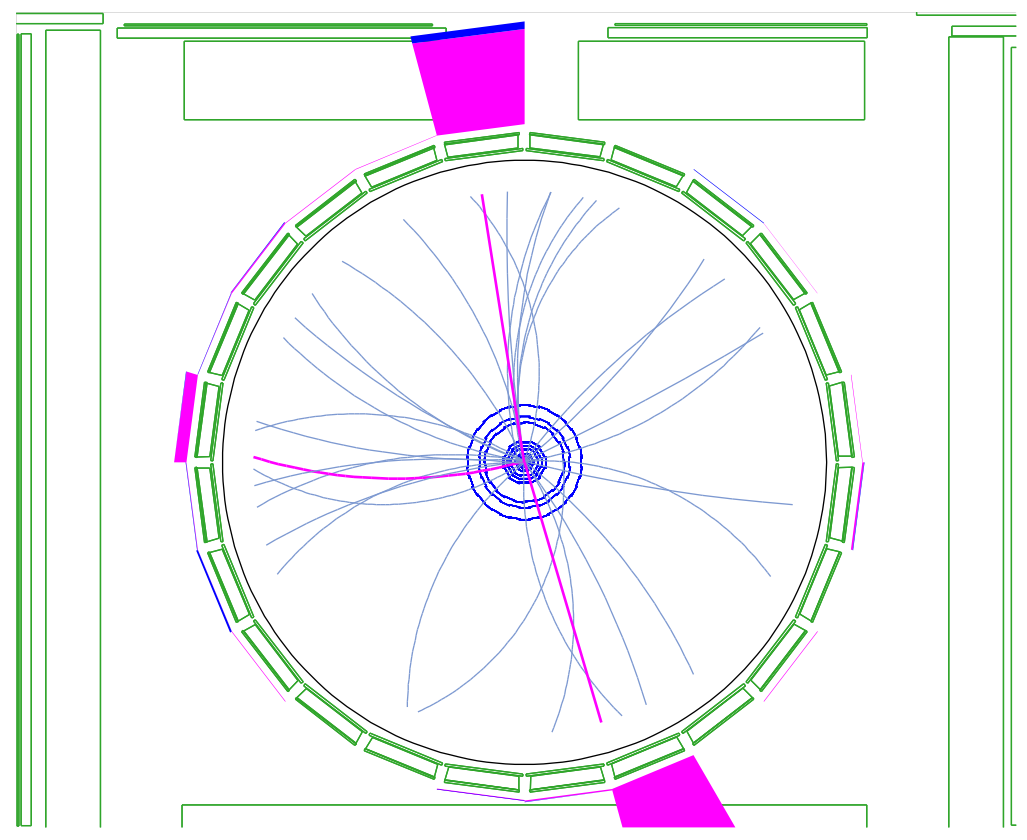

(a)

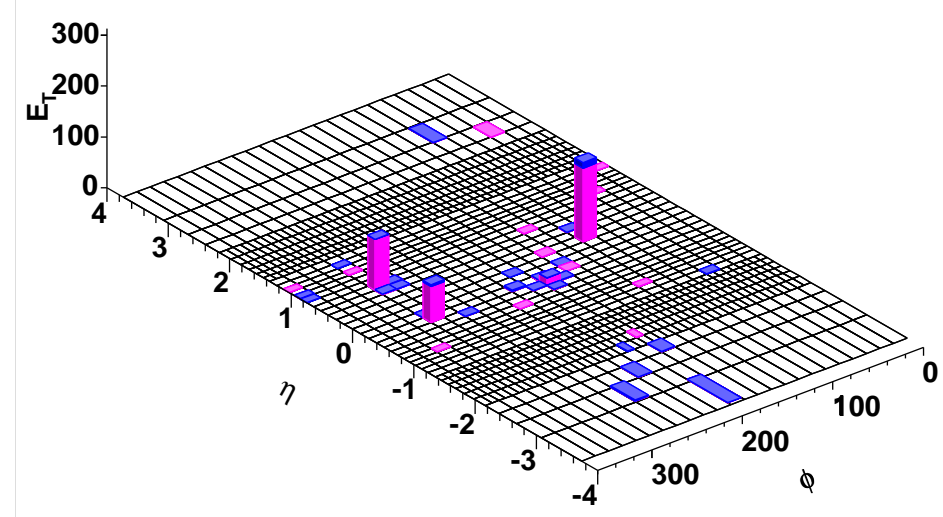

(b)

Figure 10.4: Event displays for Run $=167866$ Event $=443088$. Figure (a) shows the COT event display with track $p_{T}>0.5 \mathrm{GeV}$. Figure (b) shows the calorimeter physics towers event display with tower $E_{T}>0.5 \mathrm{GeV}$. 


\begin{tabular}{|l|c|c|c|}
\hline & $e_{1}$ & $e_{2}$ & $\gamma$ \\
\hline$E_{T}(\mathrm{GeV})$ & 165 & 96 & 73 \\
\hline$\eta_{d}$ & 0.05 & 0.53 & -0.32 \\
\hline$\eta$ & -0.03 & 0.46 & -0.29 \\
\hline$\phi$ & 1.72 & 5.00 & 5.02 \\
\hline \hline$M_{e_{1} e_{2}}$ & $M_{e_{1} \gamma}$ & $M_{e_{2} \gamma}$ & $M_{e_{1} e_{2} \gamma}$ \\
\hline $256 \mathrm{GeV}$ & $220 \mathrm{GeV}$ & $64 \mathrm{GeV}$ & $344 \mathrm{GeV}$ \\
\hline
\end{tabular}

Table 10.3: Event information for run=167866, event $=443088$.

\subsection{Integrated Background Distributions Com- pared to the Data}

We summarize this chapter by comparing the observed events to the integrated background distributions from high values to low values for $M_{e \gamma}, M_{e e \gamma}, M_{e e}$, and $E_{T}^{\gamma}$. The integrated background distributions give a measure of the number of predicted events with invariant mass $\left(\right.$ or $E_{T}^{\gamma}$ ) greater than or equal to a given mass (or $E_{T}^{\gamma}$ ) value.

Figures 10.5(a) and 10.5(b) show the predicted total integrated background distributions from high mass to low mass for the $e \gamma$ and $e e \gamma$ invariant masses. Overlaid on these figures are the locations of the observed data points. Similarly, plotted in Figures 10.6(a) and 10.6(b) are the integrated background distributions for the dielectron mass $\left(M_{e e}\right)$ and the photon transverse energy $\left(E_{T}^{\gamma}\right)$.

These plots show that the number of predicted events, integrated over all mass values, is consistent with the data. However, the locations of the data entries are at values higher than predicted, making the events interesting and worthy of further study. They also indicate this is an interesting channel to continue searching as new data become available. 


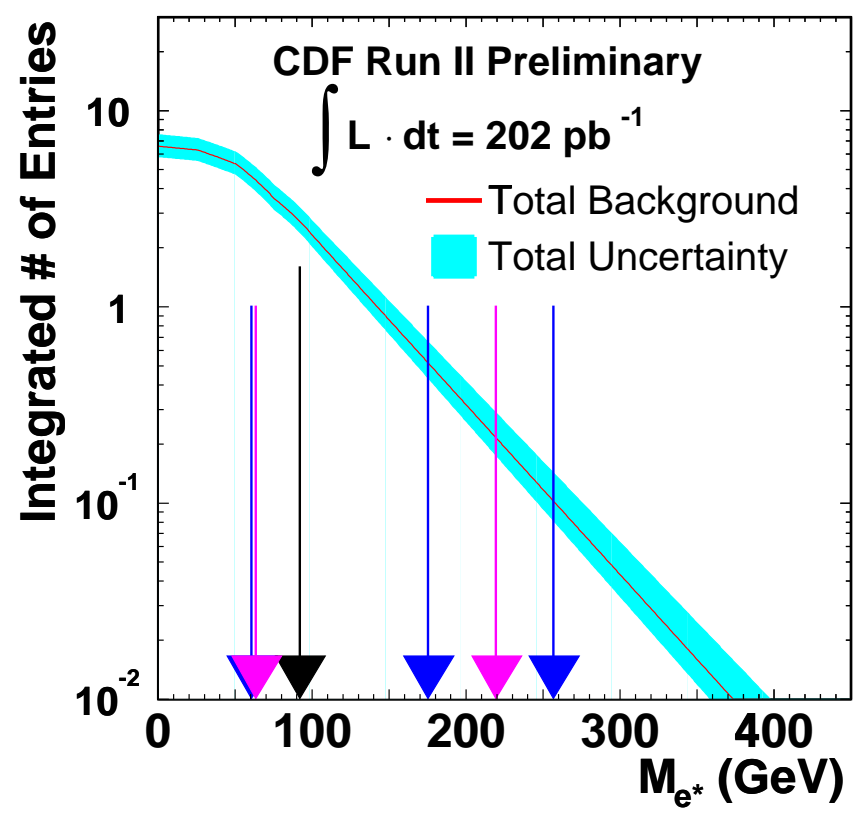

(a)

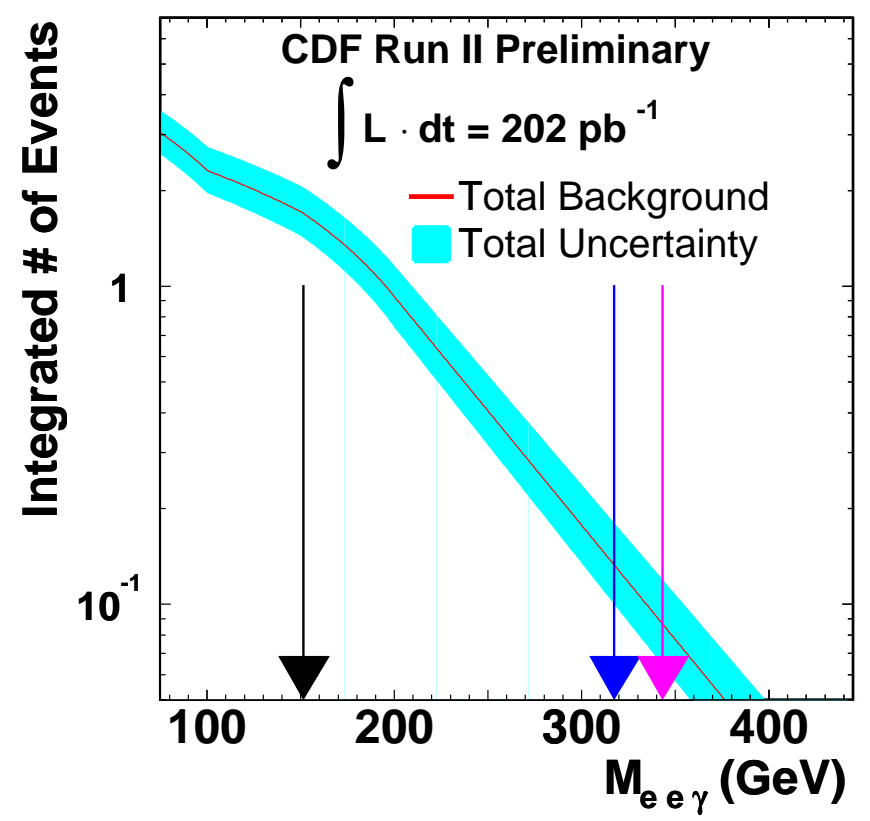

(b)

Figure 10.5: Figure (a) shows the integrated background prediction from high mass to low mass for the $M_{e *}$ distribution. Figure (b) shows the integrated background prediction from high mass to low mass for the $M_{e e \gamma}$ distribution. The observed events are overlaid in black for run $=147806$, event $=1167222$, blue for run $=144674$, event $=4143240$, and magenta for run $=167866$, event $=443088$. 


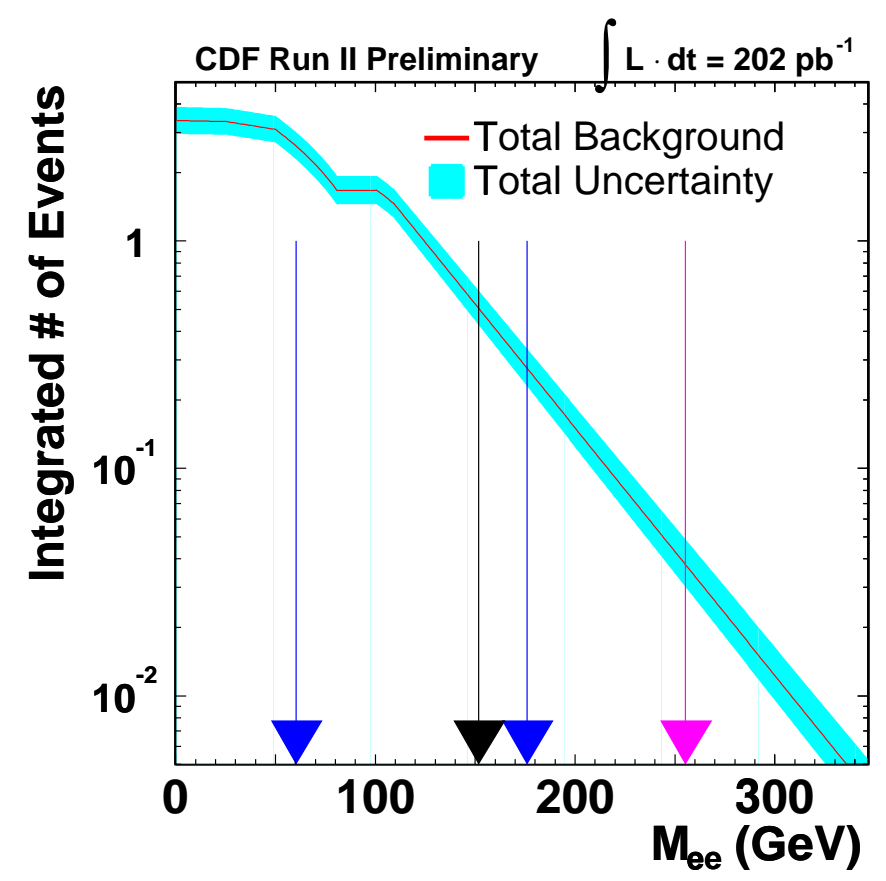

(a)

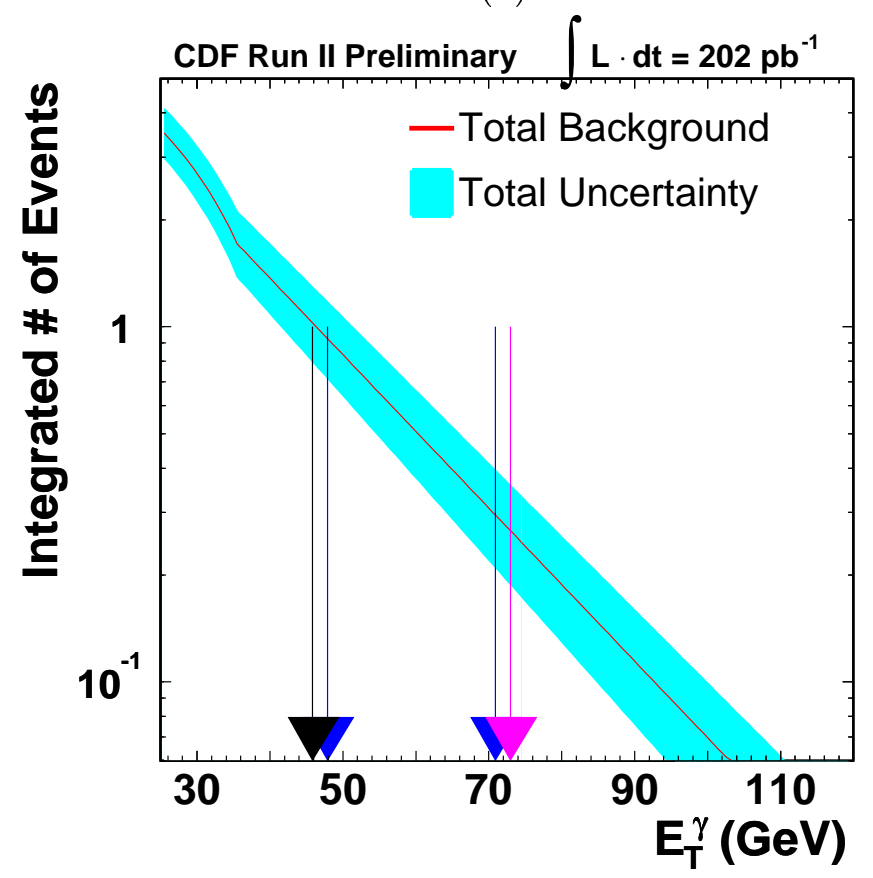

(b)

Figure 10.6: Figure (a) shows the integrated background prediction from high mass to low mass for the $M_{e e}$ distribution. Figure (b) shows the integrated background prediction from high energy to low energy for the $E_{T}^{\gamma}$ distribution. The observed events are overlaid in black for run $=147806$, event $=1167222$, blue for run $=144674$, event $=4143240$, and magenta for run $=167866$, event $=443088$. 


\section{Chapter 11}

\section{Extraction of Limits}

\section{$11.1 \quad$ Introduction}

We compare the total number of predicted background events to the number of candidate events observed in the data. From this we extract upper limits on the cross-section of models that have a dielectron + photon signature with a resonance in the electron-photon channel.

\subsection{Bayes Theorem}

The goal is to determine the upper limit on the expected signal cross-section at some confidence level, $\alpha$. The meaning of the confidence level will be discussed later in this chapter. In this analysis, the confidence level is chosen to be $95 \%$.

The upper limit on the expected signal cross-section at the $95 \%$ confidence level $\left(\sigma_{95}\right)$ is obtained by using a Bayesian approach to determine the upper limit on the number of expected events, $N_{95}$. This can be related to $\sigma_{95}$ by:

$$
\sigma_{95} \cdot B R\left(e^{*} \rightarrow e \gamma\right)=\frac{N_{95}}{\mathcal{A} \cdot \int \mathcal{L} \cdot d t}
$$

where $\mathcal{A}$ is the total signal acceptance and $\int \mathcal{L} \cdot d t$ is the total integrated luminosity 
of the data sample.

The following sections describe Bayes theorem and its implementation for finding $N_{95}$.

\subsubsection{Definition}

Bayes theorem (Equation 11.2)

$$
P(\Theta \mid \text { Data }) \propto P(\text { Data } \mid \Theta) \cdot P(\Theta)
$$

states that the probability of some hypothesis or theory $\Theta$ given the observed Data, $P(\Theta \mid$ Data $)$, is proportional to the probability of the observed Data given the theory $\Theta, P($ Data $\mid \Theta)$, times the probability of the theory $\Theta . P($ Data $\mid \Theta)$ is also known as the "likelihood function". The normalization factor for Equation 11.2 is simply $\int_{-\infty}^{\infty} P($ Data $\mid \Theta) \cdot P(\Theta) d \Theta$.

Equation 11.2 can be easily derived. Let $P(A B)$ be the probability that both A and $\mathrm{B}$ occur, then:

$$
P(A B)=P(A) \cdot P(B \mid A)
$$

where $P(A)$ is the probability A will happen and $P(B \mid A)$ is the probability of $\mathrm{B}$ given that A has occurred. Likewise, one can write the a-priori probability that the compound event $\mathrm{B}$ and $\mathrm{A}$ will occur as:

$$
P(B A)=P(B) \cdot P(A \mid B)
$$


Thus, the probability of A given that B has occurred is:

$$
P(A \mid B)=P(B A) / P(B)=P(B \mid A) \cdot P(A) / P(B)
$$

where $P(B)$ is determined by the law of total probability. The law of total probability is defined as $P(B)=\sum_{i} P\left(B \mid A_{i}\right) P\left(A_{i}\right)$, where each $A_{i}$ are mutually exclusive and the sum of all $A_{i}$ form the entire sample space, $\sum_{i} P\left(A_{i}\right)=1$. Then Equation 11.5 becomes:

$$
P(A \mid B)=P(B A) / P(B)=\frac{P(B \mid A) \cdot P(A)}{\sum_{i} P\left(B \mid A_{i}\right) P\left(A_{i}\right)}
$$

So, in Equation 11.2, we have let B equal the observed data and A equal some parameter or theory, $\Theta$, to give us the posterior probability density function, $P(\Theta \mid$ Data $) . P(\Theta \mid$ Data $)$ depends on the prior probability of $\Theta$ which specifies the probability, or degree of belief, of $\Theta$ prior to the measurement. The choice of $P(\Theta)$ requires some assumptions and this choice affects the resulting $P(\Theta \mid$ Data $)$. The convention used for CDF limits is to let $P(\Theta)$ be uniform in the physically allowed region [33], [34].

\subsubsection{Bayes Method Without Uncertainties}

We now use Bayes theorem to determine the experimental upper limit on the number of expected signal events. For simplicity, we first describe the method in the absence of uncertainties. The posterior probability function for some theory or parameter, $\Theta$, given the data, $\mathbf{x}$, is:

$$
P(\Theta \mid \mathbf{x}, \nu)=\frac{L(\mathbf{x} \mid \Theta, \nu) \cdot P(\Theta)}{\int_{-\infty}^{\infty} L(\mathbf{x} \mid \Theta, \nu) \cdot P(\Theta) d \Theta}
$$


Let $\mathbf{x}$ be the number of observed events, $n_{\circ} . \Theta$ is the parameter of interest, the number of expected signal events $(N) . \nu$, often called a nuisance parameter, is a parameter other than the parameter of interest on which the model depends. In this case, the nuisance parameter is the predicted background $(B)$. Both $B$ and $N$ are Poisson variables. Then, the likelihood function is:

$$
L(\mathbf{x} \mid \Theta, \nu)=L\left(n_{\circ} \mid N+B\right)=\frac{(N+B)^{n_{\circ}}}{n_{\circ} !} e^{-(N+B)}
$$

We define $P(\Theta)$ to be uniform in the physical region:

$$
P(\Theta)=P(N)=\begin{aligned}
& 1, N \geq 0 \\
& 0, N<0
\end{aligned}
$$

\section{Confidence Level}

The interpretation of the confidence level is that $N_{\alpha}$ is the number of expected signal events such that for $N<N_{\alpha}$, the area under the $P\left(N \mid n_{\circ}, B\right)$ distribution contains fraction $\alpha$ of the full area. For example, if $\alpha=0.95,95 \%$ of the area under the $P\left(N \mid n_{\circ}, B\right)$ curve is located below $N_{95}$ and $5 \%(1-\alpha)$ is located above $N_{95}$, the excluded region. The meaning of confidence level is further demonstrated in Figure 11.1. The parameters chosen to make Figure 11.1 are described in Section 11.2.3.

Using Equations 11.7 through 11.9, we can then determine the upper limit, $N_{\alpha}$, on the number of expected signal events, $N$, at some confidence level $\alpha$ by varying $N_{\alpha}$ until the following relation is satisfied: 


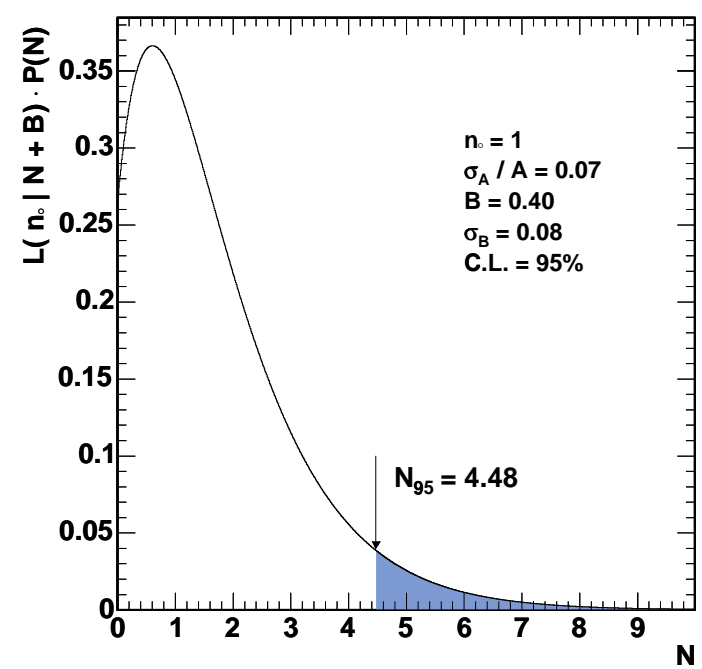

Figure 11.1: This figure is an example of the confidence level. The region $N>N_{95}$ is excluded, where $N$ is the number of expected signal events.

$$
\alpha=\int_{-\infty}^{N_{\alpha}} P\left(N \mid n_{\circ}, B\right) d N=\frac{\int_{-\infty}^{N_{\alpha}} L\left(n_{\circ} \mid N+B\right) P(N) d N}{\int_{-\infty}^{\infty} L\left(n_{\circ} \mid N+B\right) P(N) d N}=\frac{\int_{0}^{N_{\alpha}} \frac{(N+B)^{n_{\circ}}}{n_{\circ} !} e^{-(N+B)} d N}{\int_{0}^{\infty} \frac{(N+B)^{n}}{n_{\circ} !} e^{-(N+B)} d N}
$$

\subsubsection{Bayesian Method Incorporating Uncertainties}

In practice, the uncertainties are not zero. To account for this, we define $N^{\prime}=$ $N \pm \delta_{N}$ and $B^{\prime}=B \pm \delta_{B}$, where $N$ and $B$ are the mean values of $N^{\prime}$ and $B^{\prime} . \delta_{N}$ and $\delta_{B}$ are Gaussian random numbers with mean 0 and RMS $\sigma_{N}$ and $\sigma_{B}$, respectively. Then, we have:

$$
L\left(n_{\circ} \mid N+B\right) P(N)=\frac{1}{2 \pi \sigma_{B} \sigma_{N}} \int_{0}^{\infty} \int_{0}^{\infty} L\left(n_{\circ} \mid N^{\prime}+B^{\prime}\right) e^{-\frac{\left(B-B^{\prime}\right)^{2}}{2 \sigma_{B}^{2}}} e^{-\frac{\left(N-N^{\prime}\right)^{2}}{2 \sigma_{N}^{2}}} d B^{\prime} d N^{\prime}
$$


where the $L\left(n_{\circ} \mid N^{\prime}+B^{\prime}\right)$ is the usual Poisson distribution:

$$
L\left(n_{\circ} \mid N^{\prime}+B^{\prime}\right)=\frac{\left(N^{\prime}+B^{\prime}\right)^{n_{\circ}}}{n_{\circ} !} e^{-\left(N^{\prime}+B^{\prime}\right)} .
$$

By Bayes theorem, the posterior probability density function is:

$$
P\left(N \mid n_{\circ}, B\right)=\frac{L\left(n_{\circ} \mid N+B\right) P(N)}{\int_{-\infty}^{\infty} L\left(n_{\circ} \mid N+B\right) P(N) d N} .
$$

Finally, the upper limit on the number of expected signal events, $N_{\alpha}$, at some confidence level $\alpha$ is determined by:

$$
\alpha=\int_{-\infty}^{N_{\alpha}} P\left(N \mid n_{\circ}, B\right) d N
$$

In Equation 11.11, $\sigma_{B}$ comes from the predicted background uncertainty. $\sigma_{N}$ comes from the relative error on the acceptance $\left(\sigma_{A} / A\right)$ such that $\sigma_{N}=\left(\sigma_{A} / A\right) N$ [33]. Figure 11.1 shows $L\left(n_{\circ} \mid N+B\right) \cdot P(N)$ as a function of $N$ for $n_{\circ}=1, \sigma_{A} / A=$ 0.07 , and $B=0.40 \pm 0.08$. The location of the upper limit on $N$ at the $95 \%$ confidence level is shown at $N=4.48$. Equation 11.13 can be solved numerically for $N_{\alpha}$. This has been implemented in the bayes.f program [35].

\subsection{Background Prediction}

\subsubsection{Search Region}

We choose search regions that are $6 \sigma$ wide, where $\sigma$ is the RMS of the mass distribution. So, the search region is $M_{e^{*}} \pm 3 \cdot \sigma$.

We have generated and simulated several samples of $e^{*}$ events for both the gauge 
mediated and contact interaction model for a series of $M_{e^{*}}=\Lambda$ values. Each mass peak is fit to a Gaussian distribution to determine the observed mass mean and $\sigma$.

The theoretical width of the $e^{*}$ decay depends on the $e^{*}$ mass and compositeness scale:

$$
\Gamma_{\text {theory }} \propto M_{e^{*}}^{3} / \Lambda^{2} .
$$

For $\Lambda \geq M_{e^{*}}$, the detector resolution dominates the observed width. The contact interaction model requires $\Lambda \geq M_{e^{*}}$. However, the gauge mediated model allows $\Lambda<M_{e^{*}}$. When $\Lambda$ is sufficiently less than $M_{e^{*}}$, the theoretical width contributes to the observed width.

To account for the effect of the theoretical width, without running CdfSim for every possible combination of $M_{e^{*}}$ and $\Lambda$, we measure the $R M S_{R e s} / M_{e^{*}}$ where $R M S_{\text {Res }}$ is the energy resolution RMS. The $R M S_{\text {Res }} / M_{e^{*}}$ is determined to be $3.5 \%$. Then, the observed RMS is:

$$
R M S_{\text {Obs }}=\sqrt{R M S_{\text {theory }}^{2}+R M S_{\text {res }}^{2}} .
$$

\subsubsection{Background Contribution}

The background predictions are described in detail in Chapter 9. $Z(\rightarrow e e)+\gamma$ production is the largest background to the ee $\gamma$ signature. Additional contributions considered are $Z(\rightarrow e e)+$ jet, $W(\rightarrow e \nu) Z(\rightarrow e e), Z(\rightarrow e e) Z(\rightarrow e e)$, pure multi-jet, $t\left(\rightarrow e^{+} \nu_{e} b\right) \bar{t}\left(\rightarrow e^{-} \bar{\nu}_{e} b\right)$, diphoton + jet, and $W(\rightarrow e \nu)+$ jets.

The background prediction in the $M_{e^{*}}$ distribution of Figure 9.3 is used to determine the background in each mass window. The background and error distributions have been fit (for purposes of smoothing) for $M_{e^{*}}>100 \mathrm{GeV}$. For $M_{e^{*}}<100 \mathrm{GeV}$, we numerically add the background contributions in the histogram. For the case of 
$M_{e^{*}}=\Lambda$, the search regions and background predictions in Table 11.1 are used.

\begin{tabular}{|l|c|c|c|c|c|}
\hline$M_{e^{*}}$ & Peak & $R M S_{\text {obs }}$ & $3 \cdot R M S_{\text {obs }}$ & Range Used & Background \\
\hline \hline 100 & 100 & 3.5 & 11 & $89-111$ & $1.00_{-0.10}^{+0.13}$ \\
\hline 110 & 110 & 3.9 & 12 & $98-122$ & $0.92_{-0.09}^{+0.12}$ \\
\hline 125 & 125 & 4.4 & 14 & $111-139$ & $0.79_{-0.09}^{+0.11}$ \\
\hline 150 & 150 & 5.3 & 16 & $134-166$ & $0.55_{-0.07}^{+0.09}$ \\
\hline 175 & 175 & 6.1 & 19 & $156-194$ & $0.40_{-0.05}^{+0.08}$ \\
\hline 200 & 200 & 7.02 & 22 & $178-222$ & $0.29_{-0.04}^{+0.07}$ \\
\hline 225 & 225 & 7.9 & 24 & $201-249$ & $0.19_{-0.03}^{+0.05}$ \\
\hline 250 & 250 & 8.8 & 27 & $223-277$ & $0.13_{-0.02}^{+0.04}$ \\
\hline 275 & 275 & 9.7 & 29 & $246-304$ & $0.09_{-0.02}^{+0.03}$ \\
\hline 300 & 300 & 10.5 & 32 & $268-332$ & $0.06_{-0.01}^{+0.02}$ \\
\hline 350 & 350 & 12.3 & 36 & $312-386$ & $0.026_{-0.006}^{+0.011}$ \\
\hline 400 & 400 & 14.1 & 42 & $356-442$ & $0.012_{-0.003}^{+0.006}$ \\
\hline 450 & 448 & 15.8 & 48 & $400-496$ & $0.005_{-0.001}^{+0.003}$ \\
\hline 500 & 498 & 17.6 & 53 & $445-551$ & $0.0021_{-0.0006}^{+0.0014}$ \\
\hline 600 & 597 & 21.1 & 64 & $533-661$ & $0.0004_{-0.0001}^{+0.0003}$ \\
\hline 700 & 696 & 24.6 & 74 & $622-770$ & $<10^{-4}$ \\
\hline 800 & 793 & 28.1 & 85 & $708-878$ & $<10^{-4}$ \\
\hline 900 & 891 & 31.7 & 95 & $796-986$ & $<10^{-5}$ \\
\hline 1000 & 989 & 35.2 & 106 & $883-1095$ & $<10^{-6}$ \\
\hline
\end{tabular}

Table 11.1: Background prediction used in search regions for $M_{e^{*}}=\Lambda$. Units are in $\mathrm{GeV}$.

\subsection{Uncertainties}

\subsubsection{Background Uncertainty}

The errors on the background acceptance are described in Chapter 9. The relative error on the $Z \gamma$ background is $\sim 11 \%$, with primary contributions from luminosity, K-factor, PDFs, and extra material in the simulation. The other standard model backgrounds which are predicted by simulation are assumed to have uncertainties 
similar to $Z \gamma$. The backgrounds due to jets faking electrons and photons are measured from the data and the dominant errors are from the fake rate measurements. The effect of these errors is shown in Table 11.1 for each search region for $M_{e^{*}}=\Lambda$.

\subsubsection{Relative Error on Acceptance}

The relative uncertainty on the acceptance measurements is detailed in Sections 7.3 and 7.4. The contact interaction model acceptance has $\sigma_{A} / A=0.050$ for $M_{e^{*}}=100$ $\mathrm{GeV}$ and $\sigma_{A} / A=0.037$ for $M_{e^{*}}>100 \mathrm{GeV}$. The gauge mediated model relative uncertainty at $M_{e^{*}}=100 \mathrm{GeV}$ and $110 \mathrm{GeV}$ is 0.051 . For greater masses, the relative uncertainty is 0.038 .

\subsubsection{Additional Systematics for Limit Calculations}

A Bayesian approach is used to obtain upper limits on the experimental crosssection at the $95 \%$ confidence level $\left(\sigma_{95}\right)$, and, from that, extract the mass limits. In order to extract mass limits, we must take into account systematics in addition to those from the acceptance measurement. Because of the way in which the Bayesian program is implemented [35], these systematics must be added in quadrature with the relative error on the acceptance, resulting in a new relative error which we will call $\Delta L / L$.

\section{Luminosity}

Because of the relationship between cross-section and $\mathrm{N}$ in Equation 11.1, the error on the integrated luminosity measurement must be included as a systematic. The relative uncertainty on the luminosity measurement is $6 \%$ [36]. 


\section{Uncertainty on Theoretical Cross-section Due to PDFs}

In order to extract $e^{*}$ mass limits, there are uncertainties on the theoretical crosssection which must be accounted for. These uncertainties are due to the theoretical cross-section uncertainties from PDFs and the K-factor. They are included in $\Delta L / L$.

The PDF uncertainty on the cross-section is obtained by using a Hessian method that takes the CTEQ6L PDFs and varies 20 parameters on which the PDFs [37] depend. The effect on the cross-section of varying the parameters up and down is used as a measurement of the uncertainty on the cross-section measurement. Figure 11.2 shows the PDF uncertainty as a function of excited electron mass fit to a polynomial. The fit is used to determine the PDF uncertainty at a given mass point.

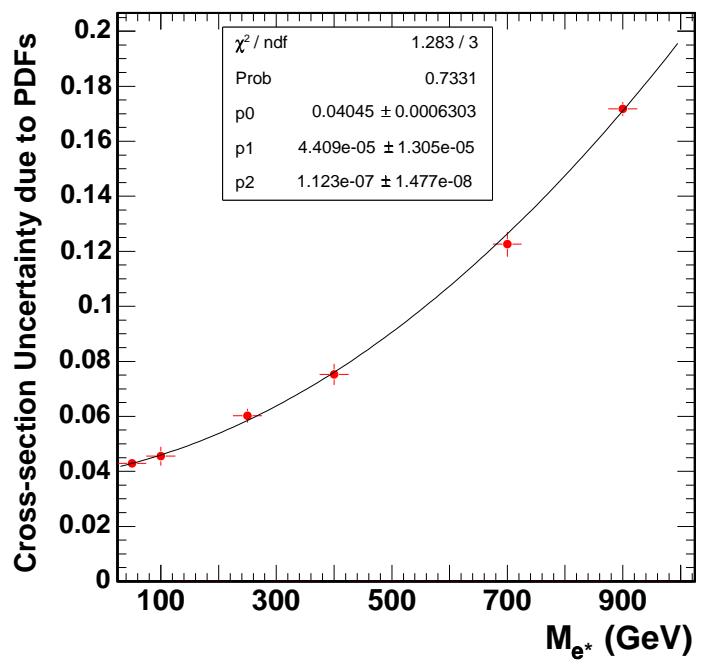

Figure 11.2: Uncertainty on $e^{*}$ cross-section due to PDFs as a function of $e^{*}$ mass. 


\section{Uncertainty on Theoretical Cross-section due to K-factor}

The method for determining the K-factor was described in Section 2.4.3. The fractional difference between the NLO and NNLO K-factors is used as the systematic error. This uncertainty is mass-dependent and is plotted for each model in Figure 11.3. The fits are used to determine the uncertainty at a given mass point.

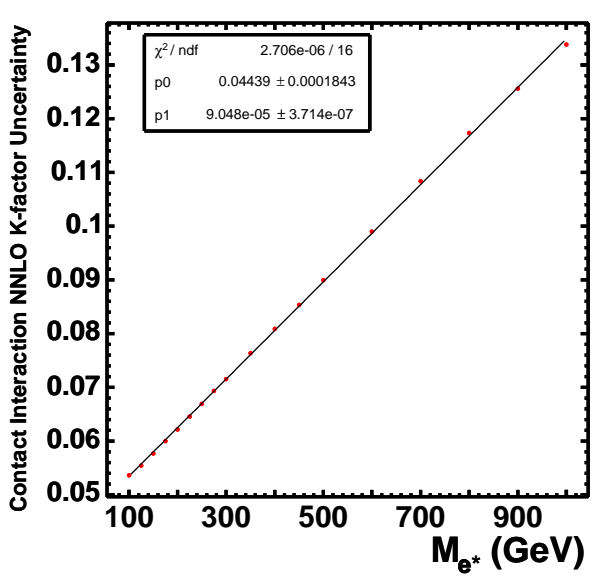

(a)

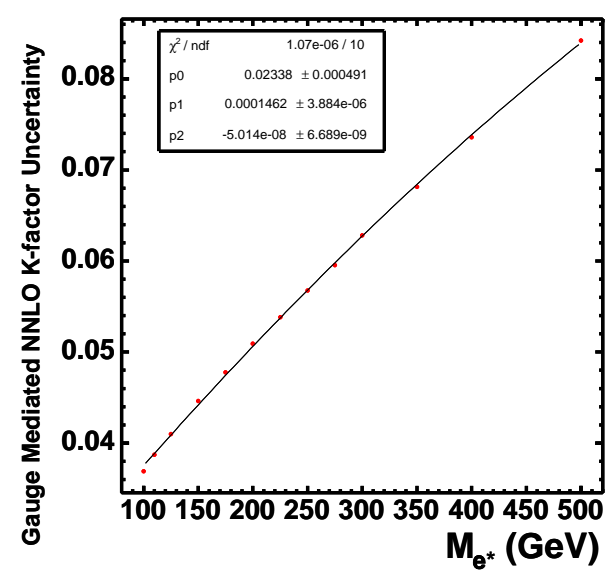

(b)

Figure 11.3: Systematic uncertainty due to K-factor as a function of $e^{*}$ mass. Figure (a) is for the contact interaction model and Figure (b) is for the gauge mediated model.

\subsubsection{Total Uncertainty Due to Acceptance, Luminosity and Theoretical Sources}

Combining the sources of uncertainty on the acceptance measurement that were discussed in Chapter 7 with the uncertainties due to luminosity, PDFs, and K-factor, we obtain the total relative uncertainty as a function of $e^{*}$ mass for each model. Figure 11.4(a) shows the total relative uncertainty for the contact interaction model. 
For $M_{e}^{*}=100 \mathrm{GeV}$, the relative uncertainty is $10.5 \%$. For $M_{e}^{*}>=125 \mathrm{GeV}$, the fit shown is used. Similarly, Figure 11.4(b) has the uncertainty on the gauge mediated model acceptance. For $M_{e}^{*}=100 \mathrm{GeV}$ and $M_{e}^{*}=110 \mathrm{GeV}$, we use $9.86 \%$ and $9.95 \%$, respectively. For higher mass values, we use the fit shown.

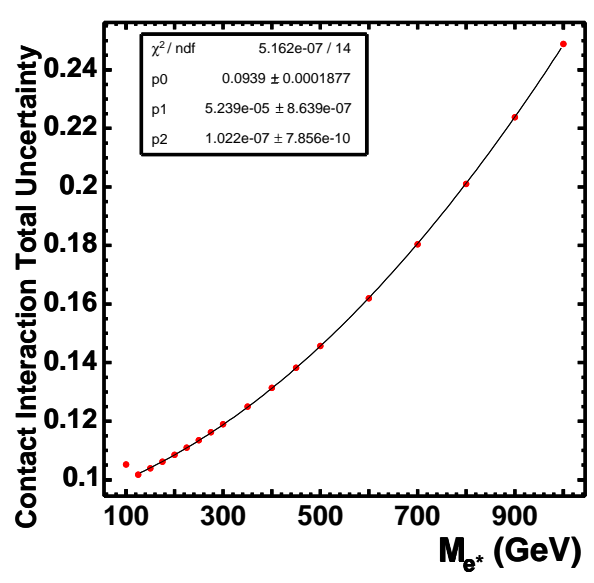

(a)

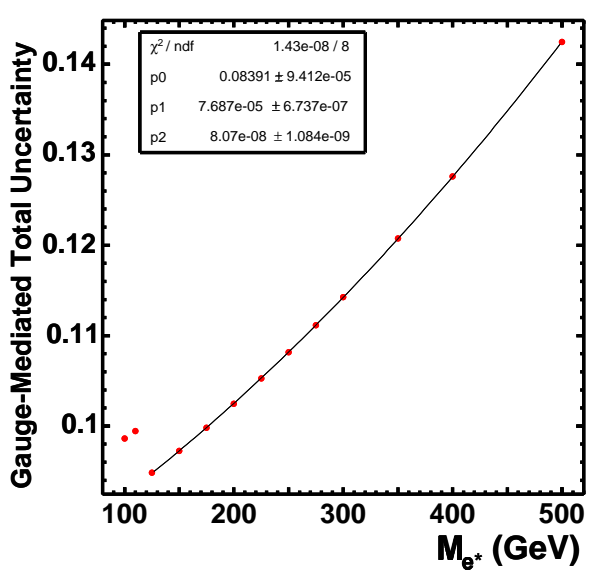

(b)

Figure 11.4: Total systematic uncertainty as a function of $e^{*}$ mass. Figure (a) is for the contact interaction model and Figure (b) is for the gauge mediated model.

\subsection{Theoretical Cross-section}

The leading order theoretical cross-sections for the contact interaction model are obtained from PYTHIA. CompHEP gives the leading order cross-sections for the gauge mediated model. These cross-sections are calculated for several $M_{e^{*}}-\Lambda$ combinations. The cross-sections are corrected by the multiplicative K-factor, which is mass-dependent (see Figure 2.14). 


\subsection{Limit Calculation}

As described in Section 11.2.3, we use a Bayesian approach to determine the upper limit on the number of expected events at the $95 \%$ confidence level. We call this $N_{95} . \quad N_{95}$ is translated to an upper limit on experimental cross-section using Equation 11.1. All values of $e^{*}$ mass and compositeness scale $\Lambda$ with theoretical cross-section greater than the upper limit on the experimental cross-section can be excluded at the $95 \%$ confidence level. $M_{e^{*}}-\Lambda$ combinations with cross-sections less than the experimental cross-section upper limit can not be excluded. Thus, the limit curves are determined by the $M_{e^{*}}-\Lambda$ combinations for which the theoretical cross-sections and the experimental cross-section limit are equal.

\subsection{Expected Mass Limits}

Prior to extracting the experimental limits, we calculate the expected mass limits based upon the background prediction and the probabilities of observing 0 through 4 events in the data. This can be used as check of whether the experimental limits are consistent with what is expected considering the background prediction, background uncertainty and acceptance uncertainty.

The expected limits are calculated for both models for the case $M_{e^{*}}=\Lambda$. The expected limit is defined by the sum of the Poisson probabilities for observing number of events between 0 and 4 :

$$
N_{e x p}=\sum_{n=0}^{4}\left(L(n \mid B) \cdot N_{95 \%}(n)\right)
$$

where $N_{\text {exp }}$ is the expected limit, $L(n \mid B)$ is the Poisson likelihood of observing 
n events given the number of predicted background events (B), $N_{95 \%}(n)$ is the Bayesian $95 \%$ confidence level upper limit on the number of events if $\mathrm{n}$ are observed. $N_{\text {exp }}$ can be related to $\sigma_{\text {exp }}$, the expected upper limit on the cross-section, by equation 11.1. Figure 11.5(a) shows the expected limit for $M_{e^{*}}=\Lambda$ in the gauge mediated model is $M_{e^{*}}>231 \mathrm{GeV}$. Figure 11.5(b) shows the expected limit for $M_{e^{*}}=\Lambda$ for the contact interaction model is $M_{e^{*}}>906 \mathrm{GeV}$.

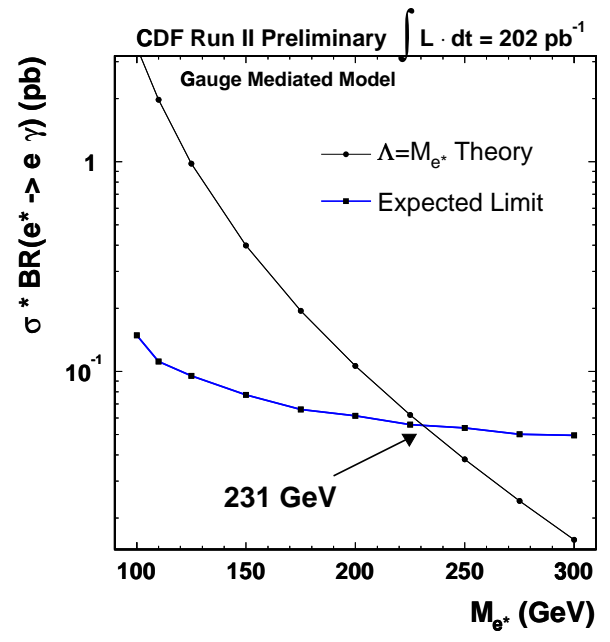

(a)

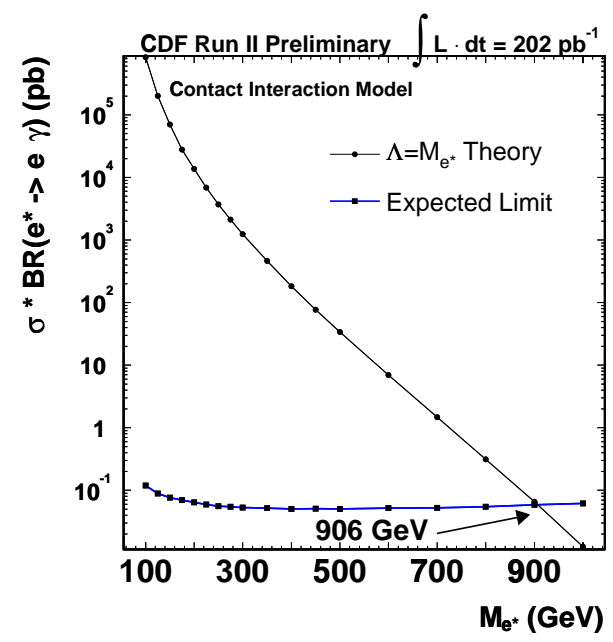

(b)

Figure 11.5: Figure (a) shows the expected limit for the gauge mediated model. Figure (b) shows the expected limit for the contact interaction model.

\subsection{Experimental Limits}

\subsubsection{Limit Results for $M_{e^{*}}=\Lambda$}

Tables 11.2 and 11.3 show the results of the upper limits on the experimental crosssection in each of the search regions for the contact interaction and gauge mediated models, respectively. There are two values of $N_{95 \%}$ and $\sigma_{95 \%}$ at each mass. The ones 
on the left correspond to a pure upper limit on the cross-section, taking into account only the relative error on the acceptance and luminosity. The $N_{95 \%}$ and $\sigma_{95 \%}$ on the right are the limits used to extract the mass limits and include uncertainties due to theoretical sources, as summarized in Section 11.4.4.

\begin{tabular}{|l|c||c|c|c||c|c|c|}
\hline$M_{e^{*}}(\mathrm{GeV})$ & $N_{\text {obs }}$ & $\Delta A / A(\%)$ & $N_{95 \%}$ & $\sigma_{95 \%}(\mathrm{pb})$ & $\Delta L / L(\%)$ & $N_{95 \%}$ & $\sigma_{95 \%}(\mathrm{pb})$ \\
\hline \hline 100 & 2 & 7.8 & 5.52 & 0.154 & 0.105 & 5.60 & 0.156 \\
\hline 125 & 0 & 7.0 & 3.04 & 0.066 & 0.102 & 3.08 & 0.067 \\
\hline 150 & 0 & 7.0 & 3.04 & 0.060 & 0.104 & 3.09 & 0.061 \\
\hline 175 & 1 & 7.0 & 4.48 & 0.085 & 0.106 & 4.56 & 0.086 \\
\hline 200 & 1 & 7.0 & 4.56 & 0.083 & 0.108 & 4.65 & 0.085 \\
\hline 225 & 1 & 7.0 & 4.63 & 0.080 & 0.111 & 4.73 & 0.082 \\
\hline 250 & 1 & 7.0 & 4.68 & 0.079 & 0.113 & 4.79 & 0.081 \\
\hline 275 & 1 & 7.0 & 4.72 & 0.079 & 0.116 & 4.84 & 0.081 \\
\hline 300 & 0 & 7.0 & 3.04 & 0.050 & 0.119 & 3.11 & 0.051 \\
\hline 350 & 0 & 7.0 & 3.04 & 0.050 & 0.125 & 3.13 & 0.051 \\
\hline 400 & 0 & 7.0 & 3.04 & 0.048 & 0.131 & 3.14 & 0.050 \\
\hline 450 & 0 & 7.0 & 3.04 & 0.049 & 0.138 & 3.16 & 0.051 \\
\hline 500 & 0 & 7.0 & 3.04 & 0.048 & 0.146 & 3.18 & 0.050 \\
\hline 600 & 0 & 7.0 & 3.04 & 0.049 & 0.162 & 3.23 & 0.052 \\
\hline 700 & 0 & 7.0 & 3.04 & 0.048 & 0.181 & 3.29 & 0.052 \\
\hline 800 & 0 & 7.0 & 3.04 & 0.049 & 0.201 & 3.39 & 0.055 \\
\hline 900 & 0 & 7.0 & 3.04 & 0.051 & 0.224 & 3.52 & 0.059 \\
\hline 1000 & 0 & 7.0 & 3.04 & 0.051 & 0.248 & 3.70 & 0.062 \\
\hline
\end{tabular}

Table 11.2: Number of observed events, relative uncertainty on acceptance and luminosity $(\Delta A / A)$, upper limit on the number of expected events using $\Delta A / A$, the upper limit on $\sigma$ at the $95 \%$ confidence level using $\Delta A / A$, total relative uncertainty $(\Delta L / L)$, upper limit on the number of expected events using $\Delta L / L$, and the upper limit on $\sigma$ at the $95 \%$ confidence level using $\Delta L / L$ for $M_{e^{*}}=\Lambda$ values between 100 $\mathrm{GeV}$ and $1000 \mathrm{GeV}$ in the contact interaction model.

The upper limit on the experimental cross-section and the theoretical crosssection curves are shown in Figure 11.6 for each model. The intersection of these curves is the $M_{e^{*}}$ limit. The gauge mediated model lower limit on $M_{e^{*}}$ is $214 \mathrm{GeV}$. This is lower than the expected limit because of the number of observed events in 


\begin{tabular}{|l|c||c|c|c||c|c|c|}
\hline$M_{e^{*}}(\mathrm{GeV})$ & $N_{\text {obs }}$ & $\Delta A / A(\%)$ & $N_{95 \%}$ & $\sigma_{95 \%}(\mathrm{pb})$ & $\Delta L / L$ & $N_{95 \%}$ & $\sigma_{95 \%}(\mathrm{pb})$ \\
\hline \hline 100 & 2 & 7.9 & 5.52 & 0.192 & 0.099 & 5.58 & 0.194 \\
\hline 110 & 0 & 7.9 & 3.05 & 0.081 & 0.099 & 3.08 & 0.082 \\
\hline 125 & 0 & 7.0 & 3.04 & 0.071 & 0.095 & 3.07 & 0.072 \\
\hline 150 & 0 & 7.0 & 3.04 & 0.061 & 0.097 & 3.07 & 0.062 \\
\hline 175 & 1 & 7.0 & 4.48 & 0.080 & 0.100 & 4.54 & 0.081 \\
\hline 200 & 1 & 7.0 & 4.56 & 0.079 & 0.103 & 4.63 & 0.081 \\
\hline 225 & 1 & 7.0 & 4.63 & 0.076 & 0.105 & 4.71 & 0.077 \\
\hline 250 & 1 & 7.0 & 4.68 & 0.073 & 0.108 & 4.77 & 0.077 \\
\hline 275 & 1 & 7.0 & 4.72 & 0.047 & 0.111 & 4.82 & 0.074 \\
\hline 300 & 0 & 7.0 & 3.04 & 0.047 & 0.114 & 3.10 & 0.048 \\
\hline
\end{tabular}

Table 11.3: Number of observed events, relative uncertainty on acceptance and luminosity $(\Delta A / A)$, upper limit on the number of expected events using $\Delta A / A$, the upper limit on $\sigma$ at the $95 \%$ confidence level using $\Delta A / A$, total relative uncertainty $(\Delta L / L)$, upper limit on the number of expected events using $\Delta L / L$, and the upper limit on $\sigma$ at the $95 \%$ confidence level using $\Delta L / L$ for $M_{e^{*}}=\Lambda$ values between 100 $\mathrm{GeV}$ and $300 \mathrm{GeV}$ in the gauge mediated model.

the region of the limit. $M_{e^{*}}<255 \mathrm{GeV}$ for $f=f^{\prime}=\Lambda / M_{e^{*}}$ is excluded by $\mathrm{H} 1$ [38].

For the contact interaction model, $M_{e^{*}}>906 \mathrm{GeV}$, consistent with the expected limit. There are no published limits for this model.

We plot the $M_{e^{*}}=\Lambda$ experimental cross-section upper limits and the $M_{e^{*}}=\Lambda$ theoretical cross-sections for for both models on the same plot in Figure 11.7.

\subsubsection{2-Dimensional Exclusion Regions}

\section{Contact Interaction Model}

The contact interaction model is valid for $M_{e^{*}} \leq \Lambda$. A natural exclusion region is the ratio of $M_{e^{*}} / \Lambda$ versus $M_{e^{*}}$. This is shown in Figure $11.9(\mathrm{a})$; region $M_{e^{*}} / \Lambda>1$ is theoretically excluded. It is also conventional to plot $1 / \Lambda$ versus $M_{e^{*}}$. This exclusion region is shown in (b) of Figure 11.9. 


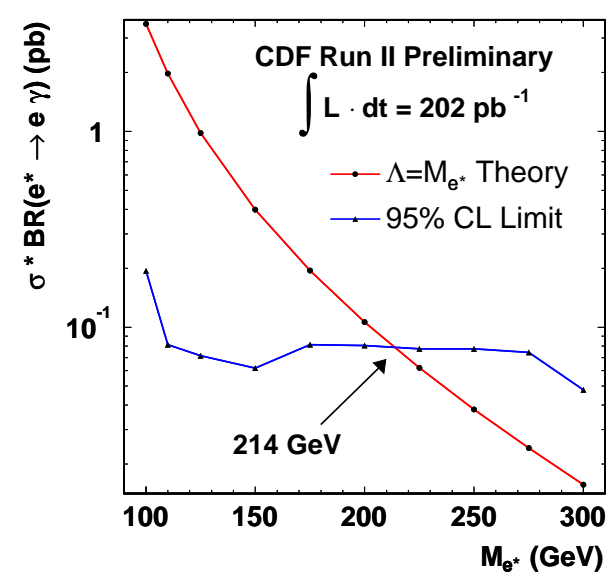

(a)

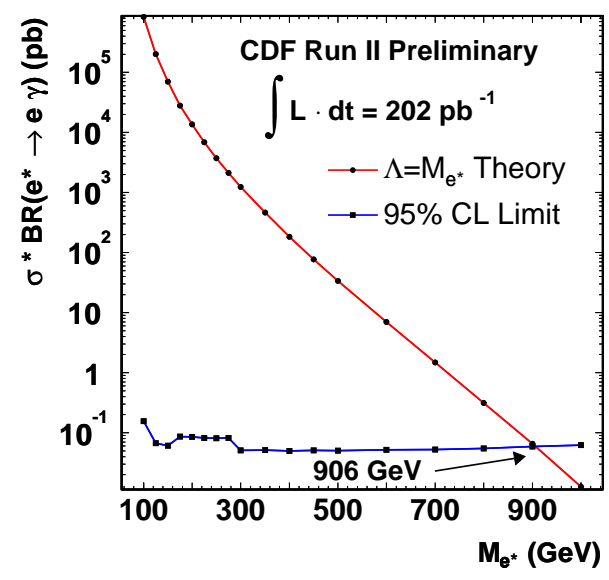

(b)

Figure 11.6: Figure (a) is the $M_{e^{*}}=\Lambda$ mass limit for the gauge mediated model. Figure (b) is the $M_{e^{*}}=\Lambda$ mass limit for the contact interaction model.

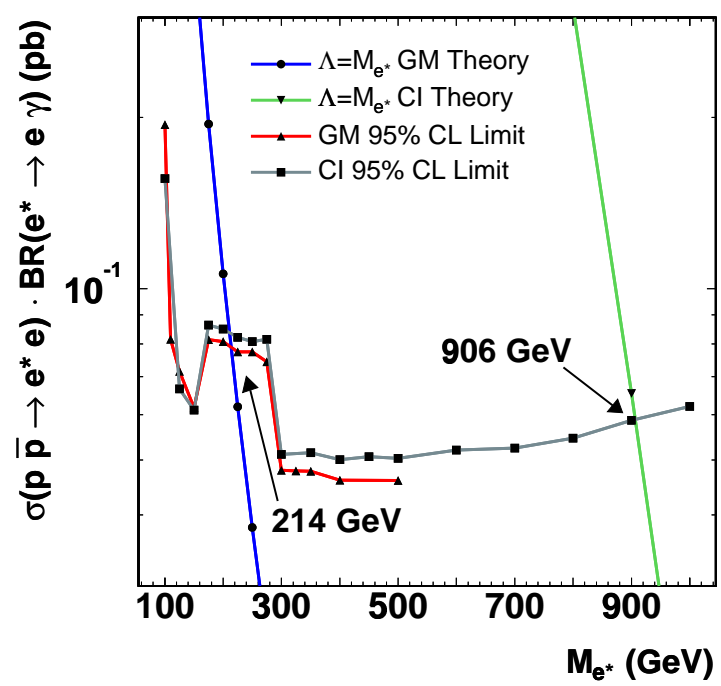

Figure 11.7: Experimental cross-section limits and theoretical cross-sections for the contact interaction and gauge mediated models, and the corresponding mass limits. 
The upper limit on the experimental cross-section and the theoretical crosssection curves are shown in Figure 11.8 for the contact interaction model for various choices of $\Lambda$. The intersections of these curves give the $M_{e^{*}}$ limits for the 2-Dimensional exclusion regions shown in Figure 11.9.

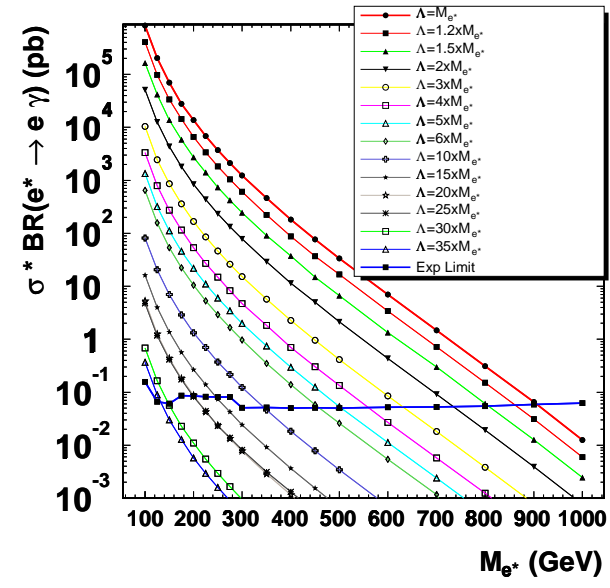

(a)

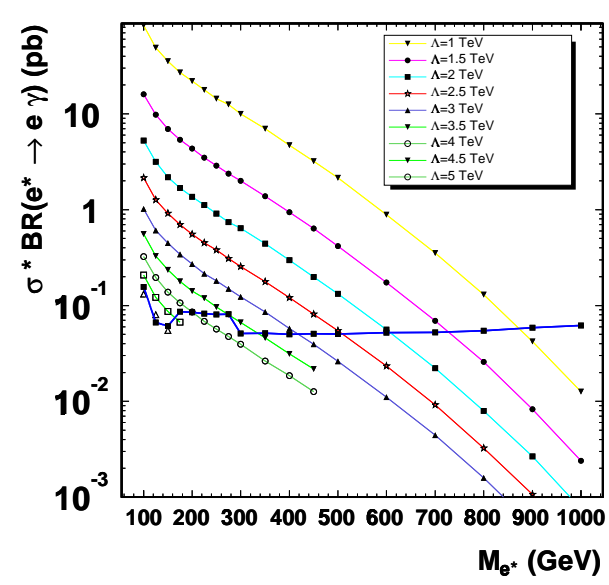

(b)

Figure 11.8: The contact interaction model theoretical cross-sections for various values of $\Lambda$ and $M_{e^{*}}$ intersecting with the $95 \%$ confidence level upper limit on the experimental cross-section.

\section{Gauge Mediated Model}

The conventional exclusion region for the gauge mediated $e^{*}$ analysis is to plot $f / \Lambda$ versus $M_{e^{*}}$. As $\Lambda<<M_{e^{*}}$, the theoretical width becomes unphysically large, rendering the gauge mediated model meaningless in this region. We choose the theoretical width equal to twice the mass of the $e^{*}$ as a cut-off $\left(\Gamma_{e^{*}}=2 \cdot M_{e^{*}}\right)$, represented by the black curve in Figure 11.10. When the experimental limit approaches the black curve, we do not extend the limit into this unphysical region.

The exclusion regions shown in Figure 11.10 are the preliminary CDF Run II 


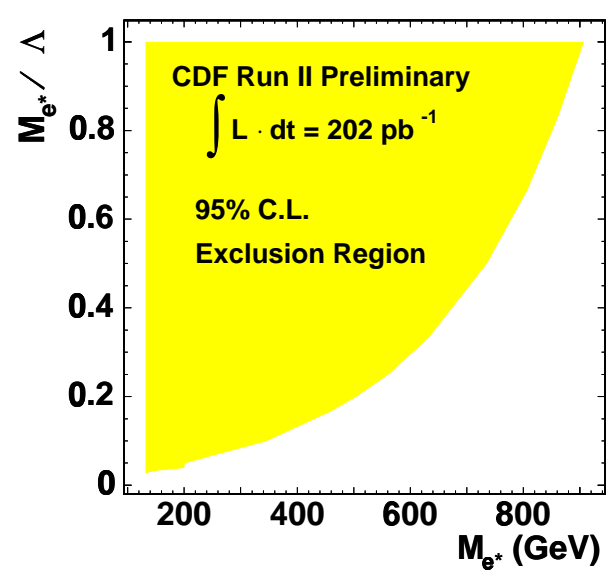

(a)

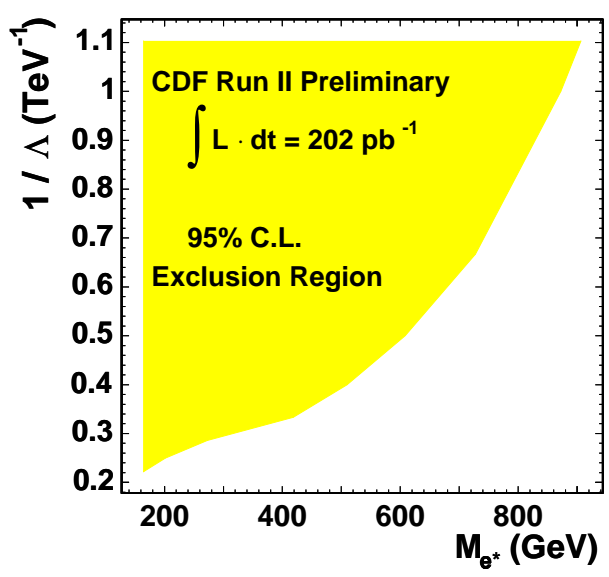

(b)

Figure 11.9: Contact interaction model exclusion regions. Figure (a) is the $M_{e^{*}} / \Lambda-M_{e^{*}}$ exclusion region. Figure (b) is the $1 / \Lambda-M_{e^{*}}$ exclusion region.

limits and the results from L3 [39], H1 [41], and ZEUS [40]. The CDF results extend sensitivity in $f / \Lambda-M_{e^{*}}$ plane for $M_{e^{*}}>280 \mathrm{GeV}$ 


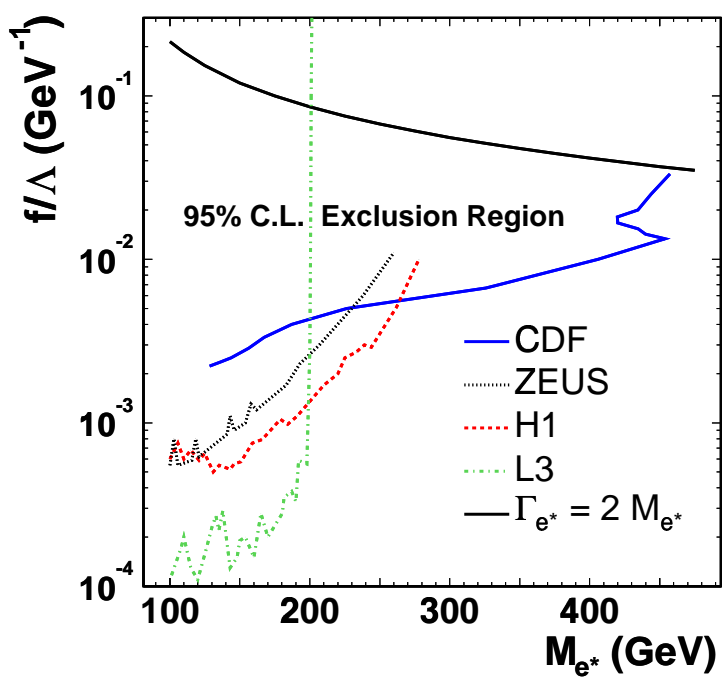

Figure 11.10: Gauge mediated model exclusion region in the $f / \Lambda-M_{e^{*}}$ plane. Shown are the exclusion regions for CDF, L3, H1, and ZEUS. The black curve is the $\Gamma_{e^{*}}=2 \cdot M_{e^{*}}$ region above which the theory is excluded. $\Gamma_{e^{*}}$ is the full theoretical width. 


\section{Chapter 12}

\section{Summary}

In this analysis, a search was performed for an excess of high energy dielectron+photon events in $\sim 202 p b^{-1}$ of data at the Fermilab CDF Run II detector. The central and forward regions of the detector were exploited in the analysis. The search focused on looking for dielectron+photon events with a resonance in the electron-photon channel. Models for excited electron production were studied because the observation of excited electrons would be an indication of lepton compositeness.

Several sources of background were predicted. Standard model $Z+\gamma$ production was the dominant background. Other background sources included $Z+$ jet, $W Z$, $Z Z$, multi-jet, $t \bar{t}$, diphoton+jet, and $W+$ jets. For the backgrounds involving jets, rates for hadronic jets to fake electrons and photons were measured. The predicted number of background events was $\sim 3$ and $\sim 6.5$ electron-photon combinations.

The efficiencies for finding central and forward electrons and photons were measured using the data. The results were compared to the CDF simulation and systematic uncertainties were determined based on the comparisons. These efficiencies were used to study the acceptances at several excited electron mass choices for two $e^{*}$ models: gauge-mediated and contact-interaction.

Having predicted $\sim 3$ events and $\sim 6.5$ electron-photon combinations and understood the efficiencies, we looked in the data for high energy eer events. Three 
events were found ( $7 \mathrm{e} \gamma$ combinations), consistent with the background estimations. However, they were unusually high energy events with interesting characteristics. For example, one of the events is a $Z Z$ candidate, in which all 4 decay electrons are observed with associated tracks. However, one electron is identified as a photon in this analysis due to the lack of tracking requirements in the plug detector, making the event appear to have the ee $\gamma$ signature of this search.

Having observed no excess of events beyond the background predictions, a Bayesian approach was used to set upper limits on the experimental cross-section at the $95 \%$ confidence level. From this, mass limits were extracted for both models for $M_{e^{*}}=\Lambda$. Additionally, 2-D exclusion regions for various $M_{e^{*}}-\Lambda$ combinations were established.

This is the first search for excited leptons at the Tevatron. We set the first limit on the contact-interaction model at $M_{e^{*}}>906 \mathrm{GeV}$ for $M_{e^{*}}=\Lambda$. For the gaugemediated model, the limit in the 2-D $f / \Lambda$ vs $M_{e^{*}}$ plane was extended to $M_{e^{*}} \sim 430$ $\mathrm{GeV}$ at $f / \Lambda \sim 10^{-2} \mathrm{GeV}^{-1}$.

The interesting events that were observed in the first $\sim 202 p b^{-1}$ of data make the dielectron+photon search signature a very exciting analysis at the Tevatron. As more data come in, we should be able to either discover or set more stringent limits on excited/exotic electron production and other models with dielectron+photon signatures. To demonstrate this, we estimate the expected limits for the gaugemediated model for $\sim 1 \mathrm{fb}^{-1}$ of data taken at $\mathrm{CDF}$. The procedure described in Section 11.7, with some modifications, is used. The increase in background and background error at higher luminosity is estimated by multiplying the central value and corresponding background error measured in this analysis by 5. Also, we consider the Poisson probabilities for up to 9 observed events rather than 4 , so that 
Equation 11.16 becomes:

$$
N_{\text {exp }}=\sum_{n=0}^{9}\left(L(n \mid B) \cdot N_{95 \%}(n)\right)
$$

The expected limit for $M_{e^{*}}=\Lambda$ is $M_{e^{*}}>324 \mathrm{GeV}$, as shown in Figure 12.1.

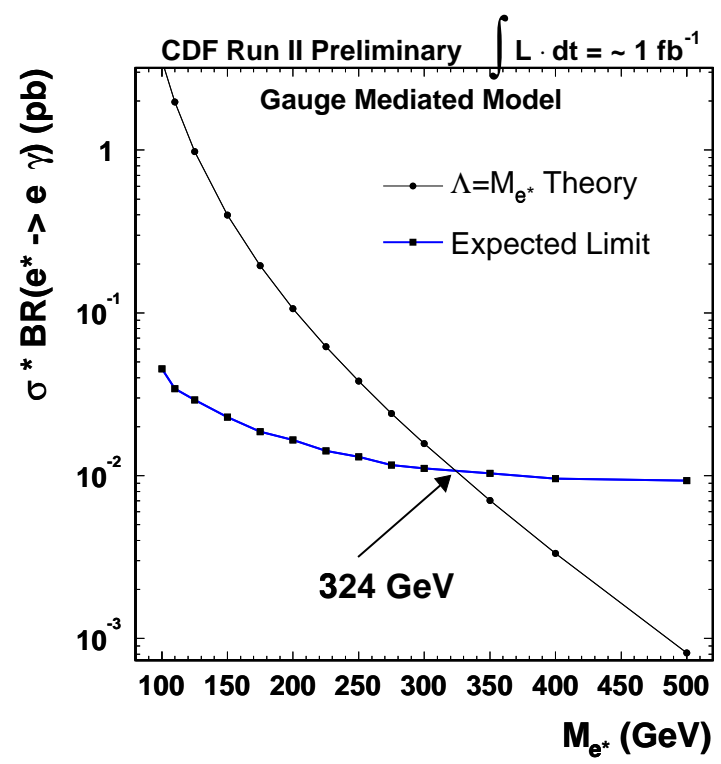

Figure 12.1: Expected limit for the gauge-mediated model for $M_{e^{*}}=\Lambda$ using $\sim 1 \mathrm{fb}^{-1}$ of data collected at CDF.

In addition to continuing this analysis at the Tevatron, the higher center of mass energy and greater luminosity potential at the large hadron collider (LHC) will greatly improve the sensitivity of this search. 


\section{Bibliography}

[1] D. Griffiths, Introduction to Elementary Particles, John Wiley \& Sons, 1987.

[2] K. Hagiwara et al. (Particle Data Group), Phys. Rev. D 66, 010001 (2002) (URL: http://pdg.lbl.gov).

[3] U. Baur, M. Spira and P. M. Zerwas, Excited-quark and Lepton Production at Hadron Colliders, Phys. Rev. D 42, 3, 815 (1990).

[4] K. Hagiwara, D. Zeppenfeld, and S. Komamiya, Excited Lepton Production at LEP and HERA, Z. Phys. C 29, 115 (1985).

[5] F. Boudjema, A. Djouadi, and J. L. Kneur, Excited Fermions at $e^{+} e^{-}$and eP Colliders, Z. Phys. C 57, 425 (1992).

[6] F. Halzen and A. Martin, Quarks \& Leptons: An Introductory Course in Modern Particle Physics, John Wiley \& Sons, 1984.

[7] T. Sjöstrand, L Lönnblad, S. Mrenna, P. Skands, PYTHIA 6.2 Physics and Manual, hep-ph/0108264, 2002.

[8] A. Semenov, LanHEP: A package for automatic generation of Feynman Rules in fiels theory, Version 2.0, hep-ph/0208011, 2002.

[9] A. Pukhov, E. Boos, M. Dubinin, V. Edneral, V. Ilyin, D. Kovalenko, A. Kryukov, V. Savrin, S. Shichanin, and A. Semenov, CompHEP - a package for evaluation of Feynman diagrams and integration over multi-particle phase space. User's manual for version 3.3, 9908288, 2000.

[10] U. Baur, T. Han and J. Ohnemus, Phys. Rev. D 57, 2823 (1998); R. Hamberg, W. L. Van Neerven and T. Matsuura, Nucl. Phys. B 359, 343 (1991).

[11] U. Baur, private communication.

[12] CDF Collaboration CDF II Detector Technical Design Report FERMILABPUB-96/390-E.

[13] T. Affolder et al., CDF Central Outer Tracker, CdfNote 6267. 
[14] The Trigger and Datasets Working Group, CDF Run II Trigger Table and Datasets Plan, CdfNote 4718.

[15] http://www-cdf.fnal.gov/upgrades/computing/projects/reconstruction/ electron/how-to/how-to-homepage.html

[16] R. Wagner, Electron Identification for Run II: Algorithms, CdfNote 5456.

[17] M. Coca, E. Halkiadakis, L. Nodulman, J.Proudfoot, P. Tamburello, M. Tanaka, E. Thomson, and G. Veramendi, A First look at Run 2 high $P_{T}$ Electrons, CdfNote 5803.

[18] A. Kotwal, private communication.

[19] H. Hayward, B. Heinemann, The Probability of a Jet Faking an Isolated Photon, CdfNote 6363.

[20] H. Gerberich and A. Kotwal, Jet $\rightarrow$ Photon Fake Rate Measurement using the CPR Detector, CdfNote 6368.

[21] D. Benjamin, L. Christofek, D. Errede, S. Errede, K. Hara, M. Lindgren, T. Muller, D. Neuberger, H. Sato, M. Shimojima, and R.G. Wagner, Determination of the QCD Jet $\rightarrow$ Fake Photon Background in the Run $1 \mathrm{~V}+$ gamma Data Samples, CdfNote 5439.

[22] M. Kim, R. Culbertson, and R. Blair, CPR Material Counts for CDF Run II, CdfNote 6101.

[23] R. Culbertson, private communication.

[24] B. Heinemann and H. Hayward, Update of the Measurement of the Probability of a Jet Faking an Isolated Photon, CdfNote 6838.

[25] B. Heinemann and H. Hayward, private communication.

[26] D. Benjamin, A. Goshaw, M. Kirby, B. Heinemann, N. Tanimoto, Theoretical Predictions of $W \gamma$ and $Z \gamma$ Production in Run II, CdfNote 6619.

[27] S. Chuang, E. Halkiadakis, P. Murat, A. Robson, and G. Veramendi, Measurement of the Material in the CDF Run II Tracking Volume using High- ${ }_{T}$ Electrons, CdfNote 6573.

[28] Y.K. Kim, J. Nielson, L. Tompkins and G. Veramendi, Trigger Efficiencies for High $E_{T}$ Electrons, CdfNote 6234.

[29] W.K. Sakumoto and A. Hocker, Event $\left|Z_{v t x}\right|<=60 \mathrm{~cm}$ Cut Efficiency for Run II, CdfNote 6331. 
[30] W. Badget. and P. Derwent, Event Z Vertex Efficiency as a Luminosity Correction for Run Ia, CdfNote 2703.

[31] J. Goldstein, T. Nelson, R. Snider, C. Issever, and D. Start, Silicon Tracking for Plug Electrons, CdfNote 5970.

[32] T. Nelson and D. Stuart, private communication.

[33] J. Conway and K. Maeshima, Upper Limits on Poisson Processes Incorporating Uncertainties in Acceptance and Background, CdfNote 4476.

[34] J. Conway, Calculation of Cross Section Upper Limits Combining Channels Incorporating Correlated and Uncorrelated Systematic Uncertainties, CdfNote 6428.

[35] J. Conway, http://www-cdf.fnal.gov/physics/statistics/code/bayes.f.

[36] S. Klimenko, J. Konigsberg, and T. Liss, Averaging of the Inelastic Crosssection Measured by the CDF and the E811 Experiments, CdfNote 6314.

[37] J. Pumplin et al., JHEP 0207, 012 (2002).

[38] K. Hagiwara et al., Phys. Rev. D 66, 010001 (2002) and 2003 off-year partial update for the 2004 edition available on the PDG WWW pages (URL: http://pdg.lbl.gov/)

[39] L3 Collaboration (P. Achard et al.), Search for Excited Leptons at LEP. Phys. Lett. B 568, 23-44, hep-ex/0306016, 2003.

[40] ZEUS Collaboration (S. Chekanov et al.), Searches for Excited Fermions in E $P$ Collisions at HERA, Phys. Lett. B 549, 32-47, hep-ex/0109018, 2002.

[41] H1 Collaboration (C. Adloff et al.), Search for Excited Electrons at HERA,Phys. Lett. B 548, 35, hep-ex/0207038, 2002. 


\section{Biography}

Heather Kay Gerberich was born on September 24, 1976, in Naperville, Illinois. She earned her Bachelor of Science degree from Clemson University in 1997. She received the Charles $\mathrm{H}$. Townes teaching fellowship from Duke University. In April 2000, she received her Master of Arts degree in Physics from Duke University. She is a member of the American Physical Society. 